\title{
Managing Dependencies and Constraints in Assembled Software Systems
}

\author{
By \\ Mark Northcott \\ A thesis submitted to \\ the Faculty of Graduate Studies and Research \\ in partial fulfilment of \\ the requirements for the degree of \\ Master of Computer Science
}

Ottawa-Carleton Institute for Computer Science

School of Computer Science

Carleton University

Ottawa, Ontario

September 2005

(C) Copyright

2005, Mark Northcott 


$\begin{array}{ll}\begin{array}{l}\text { Library and } \\ \text { Archives Canada }\end{array} & \begin{array}{l}\text { Bibliothèque et } \\ \text { Archives Canada }\end{array} \\ \begin{array}{l}\text { Published Heritage } \\ \text { Branch }\end{array} & \begin{array}{l}\text { Direction du } \\ \text { Patrimoine de l'édition }\end{array} \\ \begin{array}{l}\text { 395 Wellington Street } \\ \text { Ottawa ON K1A ON4 }\end{array} & \begin{array}{l}\text { 395, rue Wellington } \\ \text { Ottawa ON K1A ON4 } \\ \text { Canada }\end{array}\end{array}$

Your file Votre référence

ISBN: 0-494-10120-2

Our file Notre référence

ISBN: 0-494-10120-2

NOTICE:

The author has granted a nonexclusive license allowing Library and Archives Canada to reproduce, publish, archive, preserve, conserve, communicate to the public by telecommunication or on the Internet, loan, distribute and sell theses worldwide, for commercial or noncommercial purposes, in microform, paper, electronic and/or any other formats.

The author retains copyright ownership and moral rights in this thesis. Neither the thesis nor substantial extracts from it may be printed or otherwise reproduced without the author's permission.
AVIS:

L'auteur a accordé une licence non exclusive permettant à la Bibliothèque et Archives Canada de reproduire, publier, archiver, sauvegarder, conserver, transmettre au public par télécommunication ou par l'Internet, prêter, distribuer et vendre des thèses partout dans le monde, à des fins commerciales ou autres, sur support microforme, papier, électronique et/ou autres formats.

L'auteur conserve la propriété du droit d'auteur et des droits moraux qui protège cette thèse. $\mathrm{Ni}$ la thèse ni des extraits substantiels de celle-ci ne doivent être imprimés ou autrement reproduits sans son autorisation.
In compliance with the Canadian

Privacy Act some supporting forms may have been removed from this thesis.

While these forms may be included in the document page count, their removal does not represent any loss of content from the thesis.
Conformément à la loi canadienne sur la protection de la vie privée, quelques formulaires secondaires ont été enlevés de cette thèse.

Bien que ces formulaires aient inclus dans la pagination, il n'y aura aucun contenu manquant.

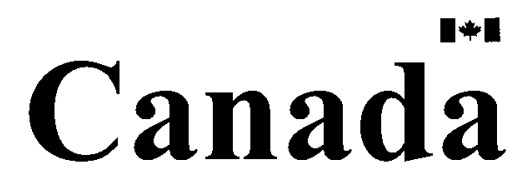




\section{Abstract}

Systems constructed by integrating off-the-shelf products are difficult to construct, deploy, and evolve because of the large number of dependencies that exist between the products. Different organizations can use different products to provide similar services, leading to different constraints at each deployed site. This results in a large number of dependencies and constraints that must be satisfied in order for the system to function properly. These dependencies and constraints are not managed in a rigorous manner often resulting in having to rediscover the information each time the system is deployed. This thesis presents an approach to managing the dependencies and constraints in software systems constructed by integration off-the-shelf products. A formal specification for modeling the products and dependencies that exist in a system is developed, and it is used as the basis for describing a process for managing dependencies and constraints. The activities that are involved in this process include defining the dependence model of the system, specifying the constraints, and manually verifying that the constraints are satisfied within the deployed system. These activities are intended to be executed concurrently by different organizations and individuals that are involved with the system. To facilitate this concurrency and geographic distribution, a tool is developed that provides support for open collaboration amongst all actors involved in the dependency and constraint management process. 


\section{Acknowledgements}

I would like to thank my supervisors, Dr. Mark Vigder and Dr. Francis Bordeleau, for their continued support throughout the writing of this thesis. Their guidance helped shape the direction of this thesis, and they contributed immensely to the completed research.

I would also like to thank the Software Engineering Group of the Institute for Information Technology and the Ontario Graduate Scholarship Program for providing funding throughout the term of this research.

Finally, I would like to thank my parents and brothers for always being there and telling me exactly what I needed to hear. 


\section{Contents}

1 Introduction $\quad 1$

1.1 Context and Motivation . . . . . . . . . . . . . 1

1.2 Problem Statement . . . . . . . . . . . . . . . . 3

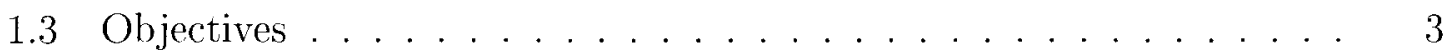

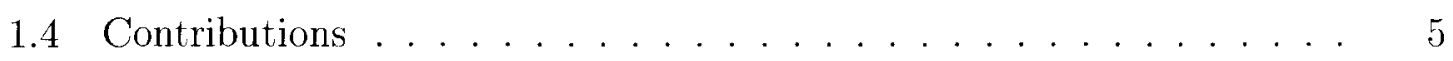

1.5 Summary ............................ 7

2 Background $\quad 9$

2.1 Identifying and Modeling Dependencies . . . . . . . . . . . . 10

2.2 Managing Dependencies and Constraints in a Software System . . . . 14

2.2.1 Software Configuration Management . . . . . . . . . . 14

2.2.2 Software Package Management . . . . . . . . . . . 16

2.2.3 Deployment and Configuration of Component-based Systems . 18

2.3 Open Collaboration . . . . . . . . . . . . . . . 21

2.4 Summary ............................ 24

3 Modeling the Software Assembly Process $\quad 26$

3.1 Software Development Through Assembly . . . . . . . . . . . . . . 28

3.1.1 Application Assembly Process . . . . . . . . . . . . . . . . . 28

3.1.2 Actors and Use Cases in the Application Assembly Process . . 30

3.2 Dependencies and Constraints in Assembled Software Systems . . . . 35

3.2.1 Dependency . . . . . . . . . . . . . . . 35

3.2 Constraint ..................... 36 
3.2.3 Discovery of Dependencies and Constraints . . . . . . . . . . 36

3.2.4 Evolution of Assembled Software Systems . . . . . . . . . . 38

3.3 Managing Constraints in Assembled Software Systems . . . . . . . . 38

3.3.1 Constraint Management Scenarios . . . . . . . . . . . . . . . 40

3.3.2 Constraint Management Requirements . . . . . . . . . . . . . 44

3.4 Need for a Formal Specification . . . . . . . . . . . . . . . . 46

3.5 Surmmary . . . . . . . . . . . . . . . . . . . 47

4 Modeling Assembled Software Systems 48

4.1 Components . . . . . . . . . . . . . . . . . 50

4.1 .1 Description ...................... 50

4.1 .2 Definition ...................... 52

4.1.3 Graphical Notation . . . . . . . . . . . . . . . 52

4.2 Dependencies .......................... 52

4.2 .1 Description ..................... 52

4.2 .2 Definition ..................... 53

4.2 .3 Graphical Notation . . . . . . . . . . . . . . . 53

4.3 Constraints . . . . . . . . . . . . . . . . . . 54

4.3 .1 Description ...................... 54

4.3 .2 Definition ..................... 54

4.3.3 Graphical Notation . . . . . . . . . . . . . . . 55

4.4 Assemblies . . . . . . . . . . . . . . . . . . . . . . 55

4.4 Description ......................... 55

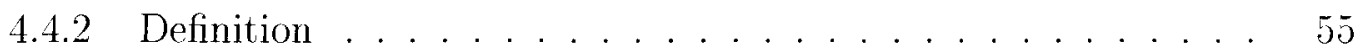

4.4 .3 Graphical Notation . . . . . . . . . . . . 57

4.5 Configurations . . . . . . . . . . . . . . . . . . 57

4.5.1 Description ...................... 57

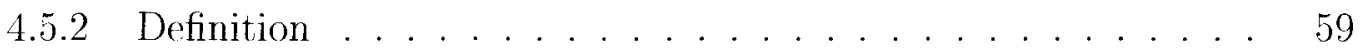

4.5.3 Graphical Notation . . . . . . . . . . . . . . 65

4.6 Specializations . . . . . . . . . . . . . . . . . . . . 66

4.6 .1 Description . . . . . . . . . . . . . 66 
4.6 .2 Definition ..................... 72

4.6 .3 Graphical Notation . . . . . . . . . . . . . . . 84

4.7 Specialization Scheme . . . . . . . . . . . . . 85

4.7.1 Description ................... 85

4.7 .2 Definition ...................... 85

4.7 .3 Graphical Notation . . . . . . . . . . . . . . . . . . 88

4.8 Discussion . . . . . . . . . . . . . . . . . . . . . . . . . . . . . 90

4.9 Summary ......................... . . . . 93

5 Managing Dependencies and Constraints $\quad 94$

5.1 Define the System Structure . . . . . . . . . . . . . . . . . 96

5.1 Base Configuration . . . . . . . . . . . . . 97

5.1.2 Example: xPetstore Base Configuration . . . . . . . . . . . 98

5.1 .3 Creating Specializations . . . . . . . . . . . 102

5.1.4 Example: Creating Specializations for the xPetstore System . 103

5.2 Specifying Constraints . . . . . . . . . . . . . . 105

5.2 .1 Declaring Constraints . . . . . . . . . . . . . 115

5.2.2 Example: Declaring Constraints in the xPetstore System . . . 116

5.2 .3 Define Constraints . . . . . . . . . . . . . . . 117

5.2.4 Example: Defining Constraints in the xPetstore System . . . . 122

5.3 Verifying Configurations . . . . . . . . . . . . . . . 124

5.4 Sharing System Structure and Constraint Information . . . . . . . . . 125

5.5 Dependency Management Tool . . . . . . . . . . . . . . . . . 126

5.5 .1 Tool Functionality . . . . . . . . . . . . . 126

5.5 .2 Tool Improvements . . . . . . . . . . . . . . . . . . . 127

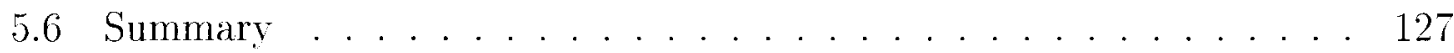

6 Case Study: Witan Web 129

6.1 About Witan Web ....................... 132

6.2 Dependency and Constraint Management Tool . . . . . . . . . . . 133

6.3 The Witan Web System Structure . . . . . . . . . . . . . . . . . 133

6.3.1 Overview of the Base Configuration . . . . . . . . . . . 134

vii 
6.3.2 WitanRootAssembly . . . . . . . . . . . . . 136

6.3.3 WitanWebAssembly. . . . . . . . . . . . . 138

6.3.4 WitanWebConfigAssembly ............... 144

6.3.5 EntityModuleAssembly . . . . . . . . . . . . 146

6.3.6 EntityModuleConfigAssembly . . . . . . . . . . . . 148

6.3.7 SecureSessionModuleAssembly .............. 151

6.3 .8 SecureSessionModuleConfigAssembly . . . . . . . . . 153

6.3 .9 InsecureSessionModuleAssembly . . . . . . . . . . 155

6.3 .10 InsecureSessionModule ConfigAssembly . . . . . . . . . . 157

6.3 .11 WebModuleAssembly ................. 159

6.3 .12 WebModuleConfigAssembly ............. 163

6.3.13 DeployEnvAssembly . . . . . . . . . . . . . 167

6.3.14 DatabaseAssembly ... . . . . . . . . . . . . 171

6.4 Selecting a View for the Witan Web Product . . . . . . . . . . . . . 172

6.4 .1 JSP View . . . . . . . . . . . . . . . . . . . 174

6.4 .2 Velocity View . . . . . . . . . . . . . . . . 177

6.4.3 Results for Selecting Different Views . . . . . . . . . . . . 182

6.5 Deploying the Witan Web System on Different Application Servers . 184

6.5 .1 JBoss $3 \ldots \ldots \ldots$. . . . . . . . . . . . . . . . 186

$6.5 .2 \quad J B o s s \quad \ldots \ldots \ldots$

6.5.3 JBoss Specific Deployment Descriptors . . . . . . . . . . . 191

6.5.4 Results for Selecting Different Application Servers . . . . . . . 196

6.6 Deploying the Witan Web System with Different Databases . . . . . . 198

$6.6 .1 \mathrm{MySQL} \ldots \ldots . \ldots . \ldots . \ldots 200$

6.6.2 PostgreSQL . . . . . . . . . . . . . . . 202

6.6.3 Results for Selecting Different Databases . . . . . . . . . . . 204

6.7 Witan Web Deployable Configurations . . . . . . . . . . . . . . 204

6.7.1 Velocity/JBoss3/MySql Deployable Configuration . . . . . 205

6.7.2 JSP/JBoss4/PostgreSQL Deployable Configuration . . . . . 211

6.7.3 Results for Different Deployable Configurations . . . . . . . . 217

6.8 Summary of Dependencies and Constraints . . . . . . . . . . . . . . 217

viii 
6.9 Discussion . . . . . . . . . . . . . . . . . . . . 221

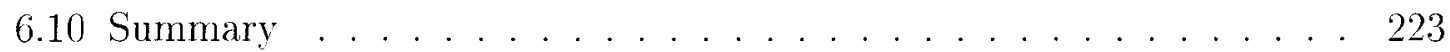

7 Conclusion $\quad 225$

7.1 Context and Motivation . . . . . . . . . . . . . . 225

7.2 Problem Statement . . . . . . . . . . . . . . 226

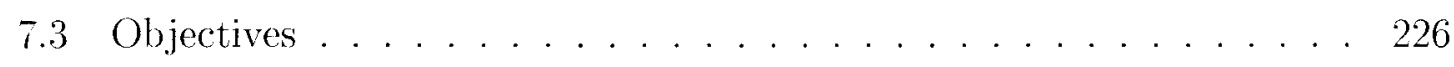

7.4 Contributions . . . . . . . . . . . . . . . 227

7.5 Future Work . . . . . . . . . . . . . . . . 230

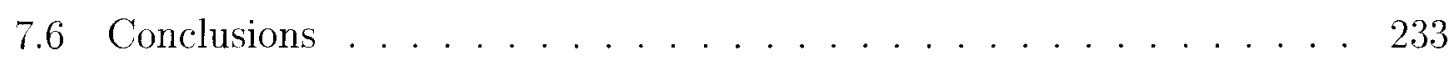

7.7 Discussion . . . . . . . . . . . . . . . . . 234

A Dependency and Constraint Management Tool 236

A.1 Tool Implementation . . . . . . . . . . . . . . . . . . . . . . . 237

A.1.1 Implementation details . . . . . . . . . . . . . . . . 238

A.2 Tool Usage. . . . . . . . . . . . . . . . . . . . 239

A.2.1 Create a New System . . . . . . . . . . . . . 240

A.2.2 System View . . . . . . . . . . . . . . . . . . 240

A.2.3 Configuration View . . . . . . . . . . . . . . . 244

A.2.4 Specialize an Existing Configuration . . . . . . . . . . 245

A.2.5 Assembly View . . . . . . . . . . . . . . . . . . . 246

A.2.6 Dependency View . . . . . . . . . . . . . . . . . 248

A.2.7 Add New Constraint . . . . . . . . . . . . . . . . . . 248

A.2.8 Constraint View . . . . . . . . . . . . . . . . . . 249

A.2.9 Committing Changes ................. . . 251

A.3 Future Improvements . . . . . . . . . . . . . . . . . . . . . . . . . 251

A.4 configuration_1_0.dtd . . . . . . . . . . . . . . 252

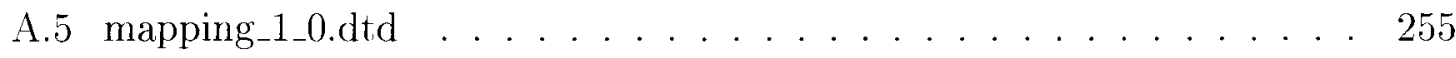

$\begin{array}{lr}\text { Bibliography } & 257\end{array}$ 


\section{List of Figures}

3.1 The use case diagram for the application assembly process. . . . . . 31

4.1 Graphical representation of each component type. . . . . . . . . . 52

4.2 The graphical representation of a dependency. . . . . . . . . . . . 54

4.3 Graphical representation of an assembly. . . . . . . . . . . . 58

4.4 Graphical representation of a configuration. . . . . . . . . . 65

4.5 Graphical representation of a configuration graph. . . . . . . . . . 66

4.6 Specialization of a configuration by replacing a virtual component with a concrete component. . . . . . . . . . . . . . . . . . . . 68

4.7 Specialization of a configuration by replacing a virtual component with a composite component. . . . . . . . . . . . . . . . . 70

4.8 Specialization of a configuration by replacing a virtual component with an existing component. . . . . . . . . . . . . . .

4.9 The graphical representation of a specialization scheme. The contents of each configuration are shown to illustrate how each specialization was created. The configuration, Configuration1, is the base configuration of this specialization scheme.

4.10 The specialization scheme graph of the specialization scheme shown in Figure 4.9. . . . . . . . . . . . . . . . . . . . . . . . . . . . 90

5.1 Base configuration for the xPetstore system. . . . . . . . . . . 100

5.2 The specializations created to obtain a deployable configuration using JBoss as the J2EE application server and HSQLDB as the database in the xPetstore system. . . . . . . . . . . . . . . . . 106 
5.3 The specializations created to obtain a deployable configuration using Orion as the J2EE application server and PostgreSQL as the database in the xPetstore system. . . . . . . . . . . . . . . . 110

5.4 The specialization scheme of the xPetstore system. . . . . . . . . 114

6.1 The configuration graph of the base configuration for the Witan Web system. . . . . . . . . . . . . . . . 136

6.2 The WitanRootAssembly assembly. This is the root assembly of the Witan Web base configuration. . . . . . . . . . . 137

6.3 The Witan WebAssembly assembly. . . . . . . . . . . . . . . . 140

6.4 The Witan WebConfigAssembly assembly. . . . . . . . . . . . . . . . 145

6.5 The entity module assembly. . . . . . . . . . . . . . . . . . 146

6.6 The entity configuration assembly. . . . . . . . . . . . . . 150

6.7 The secure session module assembly. . . . . . . . . . . . . . 151

6.8 The secure session configuration assembly. . . . . . . . . . . . . . 153

6.9 The insecure session module assembly. . . . . . . . . . . . . . . . 156

6.10 The insecure session configuration assembly. . . . . . . . . . . . . 157

6.11 The web module assembly. . . . . . . . . . . . . . . 160

6.12 The web module configuration assembly. . . . . . . . . . . . . . . 163

6.13 The DeployEnvAssembly assembly of the Witan Web system. . . . . . 168

6.14 The DatabaseAssembly assembly. . . . . . . . . . . . . . . . . . . . . 171

6.15 The WitanWebJsp configuration, which uses a JSP-based view in the web front-end. This is a specialization of the Witan WebBase configuration (Figure 6.1) . . . . . . . . . . . . . . . . . 174

6.16 The JspWebModuleAssembly assembly. The JspView component in this assembly replaces the View virtual component in WebModuleAssembly (Figure 6.11). . . . . . . . . . . . . . . . . . . 175

6.17 The JspViewAssembly assembly. This is the decomposition assembly of the Jsp View composite component in Jsp WebModuleAssembly (Figure

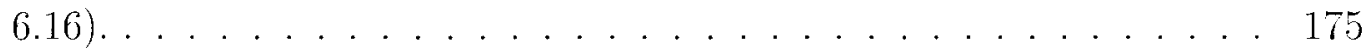


6.18 The Witan WebVelocity configuration, which uses a Velocity-based view in the web front-end. This is a specialization of the WitanWebBase configuration (Figure 6.1). . . . . . . . . . . . .

6.19 The VelocityWebModuleAssembly assembly. The VelocityView component in this assembly replaces the View virtual component in WebModuleAssembly (Figure 6.11). . . . . . . . . . . . . . . .

6.20 The VelocityViewAssembly assembly. This is the decomposition assembly of the VelocityView composite component, which is located in the VelocityWebModuleAssembly assembly (Figure 6.19). . . . . . . .

6.21 The WitanWebJBoss3 configuration, which uses JBoss v3 as the J2EE application server. This is a specialization of the WitanWebBase configuration (see Figure 6.1) . . . . . . . . . . . . . .

6.22 The JBoss3DeployEnvAssembly assembly. The JBoss3 concrete component in this assembly replaces the J2EEAppServer virtual component in DeployEnvAssembly (see Figure 6.13). . . . . . . . . . . . . . 187

6.23 The WitanWebJBoss4 configuration, which uses JBoss v4 as the J2EE application server. This is a specialization of the Witan WebBase configuration (see Figure 6.1) . . . . . . . . . . . . . . . .

6.24 The JBoss4DeployEnvAssembly assembly. The JBoss4 concrete component in this assembly replaces the J2EEAppServer virtual component in DeployEnvAssembly (see Figure 6.13). . . . . . . . . . .

6.25 The Witan WebJBoss3Config configuration, which uses JBoss v3 as the application server and includes the server specific configuration and deployment descriptor files. . . . . . . . . . . . . . . .

6.26 The WitanWebJBoss4Config configuration, which uses JBoss v4 as the application server and includes the server specific configuration and deployment descriptor files. . . . . . . . . . . . . .

6.27 The WitanWebConfigJBossAssembly assembly, which includes the JBoss server specific jboss-app.xml deployment descriptor required by the $\mathrm{Wi}$ tan Web software product. . . . . . . . . . . . . . . . 
6.28 The EntityModuleConfig.JBossAssembly assembly, which includes the JBoss server specific configuration and deployment descriptor files required within the entity module. . . . . . . . . . . . . . . . . . 194

6.29 The WitanWebConfigJBossAssembly assembly, which includes the JBoss server specific jboss-app.xml deployment descriptor required by the Witan Web software product. . . . . . . . . . . . . . . . 194

6.30 The SecureSessionModule ConfigJBossAssembly assembly. . . . . . . . 195

6.31 The IsecureSessionModule ConfigJBossAssembly assembly. . . . . . . . 195

6.32 The WebModuleConfigJBossAssembly assembly, which includes the JBoss server specific jboss-web.xml deployment descriptor required by the $\mathrm{Wi}$ tan Web software product. . . . . . . . . . . . . . . 196

6.33 The WitanWebMySql configuration, which uses MySQL as the database server and MySQL Connector/J as the JDBC driver. . . . . . . . . . 200

6.34 The MySqlAssembly assembly. The MySqlConnectorJJdbcDriver and $M y S q l D B$ concrete components in this assembly replace the $J d b c D r i v e r$ and DatabaseServer virtual components, respectively, in DeployEn$v A s s e m b l y$ (see Figure 6.14)

6.35 The WitanWebPostgreSql configuration, which uses PostgreSql as the database server. . . . . . . . . . . . . . . 203

6.36 The PostgreSqlAssembly assembly. The PostgreSqlJdbcDriver and Postgre $S q l D B$ concrete components in this assembly replace the JdbcDriver and DatabaseServer virtual components, respectively, in DeployEnvAssembly (see Figure 6.14) . . . . . . . . . . . . . . . . . 203

6.37 The Witan WebJBoss3MySqlVelocityDC deployable configuration. . . 206

6.38 The JBoss3SunJreNrCSmtpAssembly assembly. The NrcSmtpServer and SunJre concrete components in this assembly replace the EmailServer and JavaRuntime virtual components, respectively, in JBoss 3 DeployEnvAssembly (see Figure 6.22). . . . . . . . . . . . . . . . . . . . . . . . . . 208

6.39 The Witan WebJBoss4PostgreSqlJspDC deployable configuration. . . 211 
6.40 The JBoss4SunJreNrcSmtpAssembly assembly. The NrcSmtpServer and Sun.Jre concrete components in this assembly replace the EmailServer and JavaRuntime virtual components, respectively, in JBoss4DeployEnvAssembly (see Figure 6.24) . . . . . . . . . . . . . . . . . . . . 214

A.1 The architecture of the dependency and constraint managment tool. . 237

A.2 The implementation of the dependency and constraint managment tool. 238

A.3 The client view of all systems stored within the repository. . . . . 240

A.4 The form for specifying the name and description when creating a new system. . . . . . . . . . . . . . . . . . . . . 241

A.5 The form for specifying the base configuration when creating a new system. . . . . . . . . . . . . . . . . . . . 242

A.6 The client view of an existing system. . . . . . . . . . . . 243

A.7 The client view of a configuration. . . . . . . . . . . . 244

A.8 The form for specifying the XML representation of a specialization. . 245

A.9 The form for specifying the mapping from the configuration being specialized to the specialization. . . . . . . . . . . . 246

A.10 The client view of an assembly. . . . . . . . . . . 247

A.11 The client view of a dependency. . . . . . . . . . . 248

A.12 The form for specifying the name and description of a new constraint. 249

A.13 The form for specifying the verification procedure of a new constraint. 250

A.14 The client view of a constraint. . . . . . . . . . . 250 


\section{Chapter 1}

\section{Introduction}

\subsection{Context and Motivation}

Developing large scale software systems is a time consuming and resource intensive undertaking. In order to alleviate the time and resource requirements for delivering high quality software systems, organizations have moved towards a new development model. This development model focuses on assembling software products by integrating a set of existing software products [29].

The products being integrated into an assembled software product may be obtained through a variety of sources, including:

- Commercial off-the-shelf (COTS) products

- Open source libraries and frameworks

- Libraries developed by different development teams within an organization

In many cases, there will be several products available that provide the same functionality. Deciding which product to include may depend upon the requirements of the product being assembled or the context in which the assembled product is going to be used. It is possible for different instances of a product to be assembled by varying the products selected to be included within the assembled product. This is 
often the case with organizations developing product lines or frameworks, where, depending upon the requirements, different combinations of products may be integrated to produce multiple instances of the assembled product.

Typically an assembled software product is not packaged and shipped with all of the products that it requires to function properly; it may require additional services that are not included with the product in order for it to be deployed. In the simplest case, a software product requires an operating system in order to function properly. In other cases a database or application server may be required by the assembled product. Deploying the assembled product along with the additional products providing the required services results in a functional software system.

When deploying a software product, there is usually more than one product available that provides the same service. Deciding which products to use may depend on the existing environment available to the organization deploying the product or the requirements of the system. It is possible to deploy multiple instances of the same system by selecting different products to provide the services required by the product being deployed, and this is often the case when the same product is deployed by different organizations.

Assembling and deploying a software system in this manner results in a large number of dependencies between the products being integrated and deployed. Each of these dependencies may impose several restrictions, or constraints, upon the system that must be satisfied in order for the system to function properly. These constraints may include:

- A product requiring a particular directory structure be present on the file system

- A product requiring that a particular version of another product be present

- A product requiring that a configuration property be set within a configuration file

Since each product contained in an assembled software system is developed by separate development teams, these products evolve independently as features are added and bugs are fixed. In parallel to this evolution, the assembled software system 
using these products also evolves independently from the products contained within the system. As a result of this evolution, the dependencies and constraints within a software system also evolve.

It is important that these dependencies and constraints be properly managed throughout the life cycle of the software system. This is necessary in order to prevent the need to rediscover these dependencies and constraints each time that an assembled software product is deployed as a software system. Currently, it is primarily the responsibility of the developer of a software product to manage these dependencies and constraints [29]. This is accomplished by documenting them in user guides, installation instructions and frequently asked questions (FAQs) which are made available by the organization developing the product. Dependencies and constraints that have been discovered by organzations deploying the product and users of the system may be documented in an ad hoc manner through mailing lists and user groups, or even worse, they may be simply discovered and fixed, but never documented. In such cases, the constraint will have to be rediscovered by either another organization deploying the same instance of the system, or by another user that experiences the same problem.

\section{$1.2 \quad$ Problem Statement}

This thesis addresses the problem of managing the dependencies and constraints in a software product that has been assembled using a set of existing products, where there is variability in the products available, and deployed with a set of required services, which may also be variable, to produce a functional software system.

\section{$1.3 \quad$ Objectives}

This thesis attempts to solve this problem by meeting the following objectives:

1. Formally define the application assembly process: The application assembly process is the portion of the software development process that deals with assembling a software product from a set of existing software products, and deploying 
the assembled product to produce a functional system. A formal definition of this process should describe the context in which software products are assembled and deployed, as well as how dependencies and constraints may be discovered and managed.

2. Define a set of requirements for managing constraints: Using the formal definition of the application assembly process, define a set of requirements for managing the constraints within a software system. Fulfilling these requirements should result in a mechanism to simplify the constraint management process.

3. Develop a formal specification for modeling assembled software systems: To develop a formal specification for modeling the software products and dependencies in assembled software systems. This could be used as the base for providing tool support to meet the requirements of the dependency and constraint management process.

4. Define a process for modeling a system and specifying constraints: Using the mechanism for defining the structure of a software system, describe a process explaining how system may be modeled. This process should describe how the system may be modeled to accommodate selecting different software products that offer the same functionality within the system, and how the model may be specialized depending upon which products are selected. The process should also describe how constraints are specified within the system as they are discovered.

5. Provide support for open collaboration in managing constraints: To use the above process of modeling the system and specifying constraints in order to provide support for open collaboration in managing constraints. Providing this support requires a central repository for the system structure and constraint information that is accessible to all actors involved with the software system. 


\subsection{Contributions}

In order to meet the above stated objectives, this thesis presents the following contributions to the software engineering field:

\section{Formalization of the application assembly process}

The application assembly process is formalized by defining a set of use cases that describe the activities involved in assembling and deploying a software product. These use cases include:

- coding a software product by writing the source code

- selecting the software products to be used within an assembled software product and a deployed system

- assembling a software product by integrating a set of existing software products

- deploying a software product along with the products that provide the required services

- manage the constraints within a software system

Each of these use cases has one or more actors associated with it that are responsible for carrying out the use case.

\section{Requirements for managing constraints}

A series of scenarios are presented that are based upon the use cases defined in the formalization of the application assembly process. The scenarios describe different situations that may arise while carrying out the use cases, as well as the issues that arise in each situation.

A set of requirements for managing the constraints within a software system throughout the life cycle of the system are extracted from the scenarios. 


\section{Formal specification for modeling software systems}

A formal specification for modeling the software products and dependencies within a software system is defined. The formal specification allows for a system to be modeled in a manner that allows for selection points to be defincd in instances where multiple software products exist that provide the same functionality.

The formal specification also allows for a system model to be specialized to reflect the decisions made in selecting the software products to be integrated into an assembled product or deployed as part of a functional system.

Constraints may also be specified and associated with dependencies using the formal specification.

\section{Process for using the formal specification to manage dependencies and constraints}

The formal specification is used as a base for defining a process to manage the constraints in a system. The activities in the process include:

- Define the structure of a system: This involves defining the general structure of a software system with as many selection points specified as possible. It also involves specializing the system structure to reflect the software products selected to be included within the assembled product and/or deployed system.

- Specify constraints: This involves specifying the constraints that exist within a software system as they are discovered.

- Verify constraints: This involves going through all of the specified constraints and verifying that each constraint is properly satisfied, once a system has been installed. This process must be performed manually as automatic verification is considered outside the scope of this thesis.

\section{Tool support for open collaboration}

A tool was developed, as a proof of concept, for managing constraints within a software system. The tool focused on providing support for open collaboration in the 
management process. This was accomplished by providing a central repository for storing all of the system structure and constraint information. The repository is available to all actors involved with the system. and it allows for each actor to add, remove and update the constraints within the system.

\section{Apply the research to a real world example}

A case study is presented that applies the research of this thesis to the Witan Web conference management system. The case study describes how several instances of the Witan Web software product were assembled by using alternate views. Also, multiple instances of the Witan Web system were deployed by using different databases and application servers. All of the constraints within the instances of the Witan Web system presented are specified as they are discovered.

\subsection{Summary}

The remainder of this thesis is organized as follows:

Chapter 2: This chapter presents the work that has previously been done in both the research field and the software industry. The relationship between the previous work and this research is discussed, and the improvements set forth by this thesis are described.

Chapter 3: In this chapter, the application assembly process is defined using a set of use cases and actors. The dependency and constraint management use case is described in detail, and a series of scenarios that represent typical challenges faced in the dependency and constraint management process are presented. The requirements

of the dependency and constraint management process are derived from this set of scenarios and explained in detail.

Chapter 4: A formal specification is defined for modeling the products, dependencies and constraints in an assembled software system. The formalism is used for representing the hierarchical structure of a software system in terms of components, dependencies, assemblies and configurations. The formal specification provides a basis upon which tools for supporting the dependency management process may be 
built.

Chapter 5: This chapter describes how the formal specification defined in Chapter 4 may be used throughout the life cycle of a software system to assist in the dependency and constraint management process. This involves defining the structure of the system, specifying constraints within the system, and verifying existing installations of the system. An example is provided in order to help understand the activities involved in the dependency and constraint management process.

Chapter 6: The dependency and constraint management process is applied to the Witan Web conference management system. Multiple instances of the Witan Web product are assembled, and these are deployed in several different environments. The structure of the system is defined using the formal specification, and the constraints within the system are specified as they are discovered.

Chapter \%: The research presented in this thesis is summarized, and the possible future direction of the work is discussed. 


\section{Chapter 2}

\section{Background}

The process of managing dependencies and constraints that is presented in this thesis involves three areas of research:

\section{Identifying and modeling dependencies in software systems}

This deals with creating a dependence model for a software system by identifying the products and/or components within the system and the dependencies that exist between the products/components. There have been several approaches proposed in previous research for representing dependence models, as well as methods for automatically generating dependence models.

\section{Managing dependencies and constraints in software systems}

This deals with how dependencies and constraints are managed throughout the life cycle of a software system. Several areas of research have addressed this issue, including:

- Software Configuration Management (SCM): This involves managing the evolution of software artifacts within a product.

- Software package management: This involves packaging a software product in a manner such that it may be casily distributed and installed. 
- Deployment and configuration of component-based systems: This involves managing the configuration information required for deploving component-based systems

\section{Using open collaboration to facilitate the management of dependencies and constraints}

This deals with gathering input from all actors involved with a software system in the dependency and constraint management process. Open collaboration has proven successful within the open source community for developing software products, and, as a result, the concept of open collaboration is being applied to other contexts.

This chapter addresses each of these fields of research in the following sections:

Section 2.1: This section presents the previous research done in the field of identifying and defining dependencies within software systems. It also discusses how the research is related to this thesis, and the work done in this thesis to improve upon the previous research.

Section 2.2: This section presents the previous research and industrial work that has been done in the field of Software Configuration Management (SCM). The relationship between the research and this thesis is also explained.

Section 2.3: This section describes the previous work done in the field of using open collaboration to assist in the dependency and constraint management process. Also discussed is the relationship between the previous work and the research presented in this thesis.

\subsection{Identifying and Modeling Dependencies}

Assembling a software product from existing products results in a large number of dependencies. If the assembled product requires additional services in order to be deployed as a functional system, the number of dependencies increases. It is necessary to identify these dependencies and create a form of dependence model in order to assist in the development and deployment of the system. A dependence model is also helpful in managing the evolution of a software system, as it may be used to 
determine the effect that modifying a single software product has on the rest of the system. There have been several research projects that address the problem of computing a dependence model for a software system.

Vieira and Richardson present an approach for discovering and analyzing dependencies in a component-based system [45, 44]. A requirement of their approach is that a structural model of the system be explicitly defined using either the Unified Modeling Language (UML) or an Architecture Description Language (ADL). The model defines the components within the system and the relationships between the components.

The system structure model is used to create a partial-order multi-set (pomset) for each component. The pomset of a component defines the sequence of actions that occur when a request is made to a service provided by the component. The actions may be causally dependent upon other interactions within the system, or they may be concurrent, or independent, of other interactions. These concurrent interactions may occur simultaneously or in any order. As a result, the set of interactions are partially ordered, and this is the reason that partial-order multi-sets were used.

The pomsets are used to compute a Component-Based Dependence Model (CBDM) [45] for the system. The algorithm developed for computing the CBDM is as follows:

- Phase I: The required and provided services of different components are combined.

- Phase II: The individual pomset representations are composed to create a concurrent regular expression for the configuration of the system.

- Phase III: A graphical representation of the concurrent regular expression of the system configuration is created.

The CBDM is used to identify relationships between the actions within componentbased systems. This helps in determining whether changes made to one component will effect other components in the system.

In comparison to the research performed by Vieira and Richardson, this thesis also presents a specification for creating the dependence model of a system. Vieira 
and Richardson propose using partial-order multi-sets to assist in creating the dependence model, whereas this research defines a specification for modeling the software products contained within a system and the dependencies between the products. This specification also allows for selection points to be modeled in a system where two or more products exist that provide the same functionality. The model proposed by Vieira and Richardson does not take into account this case.

Also, the method for computing the dependence model of a system proposed by Vieira and Richardson requires the existence of a system model to be defined using either UML or some other ADL. This thesis does not require the existence of such a model in order to create a dependence model; however, it would be helpful in assisting with the process of defining the dependence model.

Finally, the research carried out by Vieira and Richardson is specific to componentbased systems, such as CORBA, Enterprise JavaBeans (EJBs), and COM/COM+. This thesis proposes a specification for modeling dependencies in all types of systems, with an emphasis placed on commercial off-the-shelf (COTS) based systems.

Keller et. al. describe a method for computing dependencies between software products that uses the information contained in operating system package repositories to create a dependence model [20]. Most popular operating systems contain a repository for maintaining information on which software products have been installed, as well as the dependencies between installed products. Examples of such repositories include:

- Microsoft Windows Registry

- Linux Red Hat Package Manager (RPM)

- Linux Debian Package Management System

- AIX Object Data Manager (ODM)

The approach of Keller et. al. is to parse the information stored in the repository and extract the dependency information in order to build two types of dependence models: 
1. A functional model that defines generic dependencies within a system. For example, if a database is required within a system, there would be a dependency on a database service.

2. A structural model that defines detailed dependencies that exist based upon the actual software products installed. For example, if the functional model defined a dependency upon a database service, the structural model would define the dependency information for the specific database that is installed to provide this service (ie. IBM DB2, MySQL, or Microsoft SQL Server).

Keller et. al. do not provide a formal model for representing the computed dependence models. However, they provide a system for classifying dependencies based upon several criteria such as space/locality/domain, dependency criticality, dependency formalization, dependency strength, component type and component activity. This classification assists in identifying the different aspects of dependencies.

This thesis also focuses on creating a dependence model for a software system; however, unlike the research presented by Keller et. al. which defines a method for automatically generating a dependence model, this thesis requires that the dependence model be manually defined. The reason that the dependence model must be created manually is that there is no assumption made as to the dependency information that may already be defined amongst the products that exist in the model. This thesis also develops a formal specification for defining the dependence model of a system, whereas the research of Keller et. al. only specifies how the dependence model may be computed.

The research of Keller et. al. also describes how a functional dependence model may be specified to reflect generic dependencies within the svstem that are unrelated to the products used within the system. The formal specification for defining dependence models presented in this thesis also focuses on abstracting the functionality required within the system from the actual products used within the system. This is accomplished by modeling selection points within the dependence model whenever there exists more than one product that provides the same functionality.

Finally, the research of Keller et. al. requires that there exists a repository 
for maintaining the information pertaining to which products have been installed on a machine; however, these repositories are typically operating system dependent. whereas this thesis proposes a process for defining the dependence model of a system, independent of the operating svstem, and it does not require the existence of a repository containing installed product information.

\subsection{Managing Dependencies and Constraints in a Software System}

The management of dependencies and constraints within a software system involves documenting the dependencies and constraints in a manner such that they can be made accessible to those responsible for deploying the system. This issue has been previously addressed in several contexts, each of which is related to the research presented in this thesis:

- software configuration management

- software package management

- deployment and configuration of component-based systems

\subsubsection{Software Configuration Management}

Software configuration management (SCM) is a process for controlling the evolution of all software artifacts contained within a software system. The IEEE Standard for Software Configuration Management Plans [12] defines four functions of SCM:

- Identification: identifying and naming all configuration items within a software system. Configuration items include source code files, documentation, binary executables, configuration files, etc.

- Control: controlling all changes made to configuration items. This includes documentating the need for the change, approving the change, and verifying and releasing the change. 
- Status accounting: recording and reporting the status of configuration items. This includes tracking approved versions of configuration items, the status of change requests, and the implementation status of approved changes.

- Audit and review: determining the completeness of a software system. This includes reviewing configuration items to ensure that they meet the required physical and functional characteristics.

There are a large number of SCM tools available that are used to automate the SCM process. Such tools include Rational ClearCase [33], Microsoft Visual SourceSafe [46], Concurrent Versions System (CVS) [5], and Subversion [35]. Each tool offers varying levels of support for the SCM process, but, at minimum, each tool provides the functionality for tracking different versions of a software product. Every version of a software product contains all of the source code, binary executables, documentation and configuration information that pertains to that particular version of the product. Given this functionality, it is possible to use SCM tools for managing dependencies and constraints in each version of a software product.

However, using SCM tools for managing dependencies and constraints has several drawbacks. First, SCM tools are typically used internally within an organization, and access to the repository is only granted to approved members of the development team. Therefore, any dependency and constraint information that is identified by people without access to the SCM repository, must be forwarded to a developer that has the proper permissions to update the information in the repository.

This issue is addressed within this thesis by focusing on providing support for open collaboration amongst all actors involved with a software system in order to manage the dependencies and constraints within a software system. By encouraging open collaboration it is believed that the dependency and constraint information for a system may be maintained in a more efficient manner, and the information will be kept up to date.

Another drawback of SCM tools is that they do not provide a formal structure that may be used to model dependencies in a software system. Although this is not a requirement for the management of dependencies and constraints, it is considered 
to be a useful tool to facilitate the process.

This research defines a formal specification for modeling the software products contained within a software system, as well as the dependencies between the products. The formal specification allows for selection points to be defined in instances where several products exist that provide the same functionality. This makes it possible to model a product that may be assembled using different sets of products and deployed using different products to provide the required services. This formal specification could be used in conjunction with existing SCM tools by using the SCM tool to manage the evolution of the system structure and constraint information.

Finally, SCM tools do not support the sharing of dependency and constraint information between organizations. This is important when a software product is being created by assembling products from different organizations as it allows for all of the dependency and constraint information defined for a product to be incorporated into the the assembled product. As a result, it is not necessary to rediscover the information for each product included in the assembled product.

The formal specification defined in this research is capable of providing support for sharing the dependency and constraint information between organizations. Using the Internet as a communication medium, it is possible to link the system structure and constraint information that has been defined for each product contained within a software system. This allows for the information to be shared across physical boundaries, thus preventing from having to redefine the system structure and constraint information for a product each time it is included in a larger product.

\subsubsection{Software Package Management}

Software package management involves providing support for installing, uninstalling, verifying and querying software products on a particular operating system. A software product is bundled into a software package, and each software package defines all of the other packages upon which it depends. A software package may also provide scripts that are to be executed when a package is installed or uninstalled. These scripts are used to check any constraints that may be defined within the software 
package.

A package management system is responsible for providing a repository that maintains the information of all software packages that have been installed. The repository may be queried to determine which packages have been installed, as well as the dependencies between packages. A package management system may also provide support for automatically obtaining dependent software packages when installing a package. Examples of package management systems are:

- Windows Installer Service [22]: used within the Microsoft Windows operating system.

- Red Hat Package Manager [2]: used within several Linux based operating systems, including Red Hat Enterprise, Mandrake, and Fedora Core.

- Debian Package Management System [1]: used within several Linux based operating systems, including Debian and Ubuntu. It has also been ported to the Mac OSX operating system.

- Software Release Manager [42]: implemented by van der Hoek and Wolf for software products that have been developed using component-based technologies, including EJB, .NET and the CORBA Component Model (CCM).

Each of these software package management systems address the problem of managing dependencies and constraints between software products. Software packages must define all of the dependencies of the product contained within the package, and it may also provide support for verifying that constraints are satisfied when installing and uninstalling the package.

However, software package management also has several drawbacks in managing dependencies and constraints. First, all of the products contained in a software system must be installed using the same package management system in order for the dependencies and constraints to be properly satisfied. This is often not possible, especially in distributed software systems that make use of software products installed on various operating systems. 
This research addresses this issue by providing a formal specification for modeling and managing dependencies in a software system. The formal specification allows for the system to be modeled in a manner that is independent of how the products within the system are installed. The system model is also independent of how the products within the system are distributed. This allows it to overcome the deficiencies of software package management systems.

Also, within software package management systems, all dependencies and constraints must be defined by the person responsible for packaging the software product. This is an issue because the packager may not know all of the dependencies and constraints associated with a software product.

This problem with software package management is addressed in this research by relying on open collaboration in order to allow all actors involved with the software system to contribute dependency and constraint information. Dividing the responsiblity of discovering and specifying constraints within a software system amongst all of the actors should help in defining a more complete set of constraints, and it should prevent having to rediscover constraints each time a product is deployed.

\subsubsection{Deployment and Configuration of Component-based Sys- tems}

Software systems that have been developed using component-based platforms, such as Java 2, Enterprise Edition (J2EE) [13], Microsoft .NET [23], and the CORBA Component Model (CCM) [6], need to be configured and deployed into a compliant container in order to function properly. The container is responsible for providing the services required to allow communication between the components of the system. Each platform defines a set of deployment descriptors that are used to specify deployment and configuration information for a component-based software system developed using that platform. 
In order to facilitate the deployment and configuration of component-based systems, the Object Management Group (OMG) adopted the Deployment and Configuration of Component-based Distributed Applications Specification (D \& C Specification) [7]. This specification defines a model and process for deploying and configuring component-based systems that is independent of the component platform being used. This is accomplished by using a Model Driven Architecture (MDA) [21] approach. The model defined by the specification allows for all components within a system to be specified, as well as the relationships between components. The model is defined as a Platform Independent Model (PIM). The PIM may be transformed into to a model that is specific to a particular component platform, known as a Platform Specific Model (PSM).

Using the process and model defined in the D \& C Specification, it is possible to manage the dependencies and constraints within a component-based software system. Dependencies may be represented using the model defined in the specification, and constraints may be defined as configuration properties that are associated with each component. The specification allows for multiple instances of the same system to be defined by using different sets of components within the system, and it also provides support for specifying multiple configurations for a system. These features are important in the management of dependencies and constraints in software systems.

This research is closely related to the D \& C Specification. The similarities between the two include:

- Formal specification for modeling the dependencies in a software system: Both this research and the D \& C Specification define a formal specification for modeling the products (or components) within a software system and the dependencies between the products (components). The formal specification presented in this thesis is defined in terms of a mathematical model, and it defines a graphical representation for each element in the specification. The D \& C Specification presents a formal specification that is defined using UML, and UML is also used for the graphical representation of a system model. 
Both formal specifications allow for selection points to be defined within a system in instances where multiple products (components) are available that provide the same functionality. This allows for different variations of the same system to be modeled by selecting different product (component) sets.

Constraints may also be specified using both formal specifications; however, how the constraints are specified is slightly different. Using the formal specification presented in this thesis, constraints are associated with dependencies, whercas constraints are associated with components within the D \& C Specification.

- Process for managing dependencies and constraints: This thesis and the D \& C Specification both define a process for managing the dependencies and constraints in a software system. Both processes focus on defining the structure of the system in a manner that will allow for different variations of the system to be deployed.

The system structure is then used to specify constraints within the system. The constraints that are defined are dependent upon which products (or components) are included within the system.

Some form of verification is also carried out during the process. In this research, the verification is performed manually by checking to ensure that all constraints have been satisfied once the system is installed. In the D \& C Specification, the constraints are verified within the system model as the properties defined for each component must satisfy the specified constraints in order for the system to be deployed.

In contrast, this research differs from the D \& C Specification in the following regards:

- Independent of system development model: This thesis focuses on managing the dependencies and constraints within all types of software systems. It makes no assumptions as to the development platform used to produce the system, although it places an emphasis on systems assembled using commercial offthe-shelf (COTS) products. In contast, the D \& C Specification is specific to component-based systems. It allows for platform independent models to be 
created, however, the platfrom specific models are relevant only to componentbased platforms, such as .NET. E.JB and CORBA.

- Use of open collaboration for managing dependencies and constraints: This rosearch focuses on using open collaboration amongst all actors involved with a software system for assisting in the management of dependencies and constraints. However, within the D \& C Specification, only a subset of the actors are involved with the dependency and constraint management process. Using open collaboration, it is believed that the amount of work required for rediscovering constraints each time a system is deployed will be reduced.

\subsection{Open Collaboration}

The creation of the Internet, and more recently the World Wide Web, has played a huge role in facilitating open collaboration between groups of people. The importance of open collaboration in software development has been demonstrated by the recent increase in popularity of open source software. In open source projects, developers are geographically distributed, and they must rely on methods of open collaboration in order to produce functional software products.

Early methods of open collaboration existed in the form of bulletin boards, email mailing lists, and Usenet. More recently, Internet Relay Chat (IRC), and instant messaging have been used to provide a method of open collaboration $[40,36]$. These forms of open collaboration have been used in a variety of ways to facilitate communication amongst individuals residing in geographically distributed locations. Some of these uses include:

- Sharing information amongst a group of researchers

- Sharing source code and patches in open source projects

- Documenting bugs in software products

- Documenting dependencies and constraints in software systems 
Often the same method is used to carry out more than one of these functions. For example, mailing lists are typically used in open source projects as a means of discussing bugs in the software, providing patches for bugs, and describing dependencies and constraints within the product. As a result, all of this information is located in a single location, making it difficult to find the relevant information when necessary.

This research attempts to overcome this issue by focusing strictly on providing a method for documenting the dependencies and constraints within a software system. Using a formal specification provides a structure that assists in locating the desired information when needed. Open collaboration is then used to assist in the dependency and constraint management process.

Recently, wikis have become the latest method for achieving open collaboration amongst a group of individuals [40,36]. A wiki [47] is a server-side application that allows users to modify the contents of a web page using any web browser. Any user is free to create new content or modify existing content. Users are also free to modify the structure of the content by organizing the contributions that have been made. The ideology behind the wiki is that by allowing any user the privilege to add content to the web page, it provides a sense of responsibility to the community. This responsibility works to provide a form of self-moderation as users are conscious to add only valuable content. Any content that is deemed not fitting of the web page will be removed by other members of the community. This method of selfgovernance allows for the productive open collaboration required to maintain useful and up-to-date documentation for a software product.

The use of a wiki has been embraced by several projects within the open source community for maintaining the documentation within the project, including Mono [25], Ubuntu [41] and Beagle [3]. Most important is how these projects are using the wiki to manage dependency and constraint information within the software product.

An example of an open source project using a wiki to manage dependency and constraint information is the Beagle project [4]. Beagle is a tool used for indexing and searching personal data, including documents, email, web browsing history, instant message conversations, and many other forms of data. As users experiment with 
Beagle on different platforms and in different environments, all dependency and constraint information that is discovered is added to the wiki. This allows other users to quickly resolve any issues they may encounter while trying to install and run Beagle. This open collaboration prevents individual users from having to reinvent the wheel each time they install Beagle.

However, when it comes to managing dependencies and constraints, wikis suffer from the lack of a formal specification for defining the structure of a system. Although it is possible to structure the set of web pages containing the dependency and constraint information in a logical manner that resembles a system model, there is no formalism for enforcing this structure. As a result, the dependency and constraint information may end up being defined in an ad hoc manner making it difficult to locate the information when necessary.

This thesis addresses this issue by developing a formal specification for modeling the products contained within a software system and the dependencies between the products. Constraints are then defined and associated with dependencies in the model. This formal structure makes it easier to locate the constraint information that has been defined within a system. Also, the formal specification allows for the system structure and constraint information to be shared across organizations, as it is possible to link the models defined for individual products.

Open collaboration has always been a major part of open source development; however, it is being adopted by other communities to assist in information sharing. The COTS community is an example of one such community that has tried to benefit from open collaboration. Mielnik et. al. created the eCots project with the goal of setting up a portal for collecting and improving the accuracy of information pertaining to COTS products and producers $[24,8]$.

The eCots project is based upon the standardization of COTS related data, and it addresses the issue of providing a standard structure for obtaining, qualifying and updating the information of COTS products. The eCots portal [8] presents a directory of COTS products that are available. For each product, a set of properties are defined. These properties include specifying other COTS products that are related to the product, as well as documentation available for the product. 
Although the eCots project provides a standard method for defining information pertaining to COTS products, it does not define a formal specification for modeling the dependencies between products and the constraints associated with those dependencies.

This thesis addresses this issue by focusing on how larger software products may be assembled from existing products. The assembled products are modeled using the formal specification presented in this thesis, and dependencies and constraints within the product are defined. This allows for the relationships between existing products to be defined in a formal structure.

Also, although the eCots project focuses on using open collaboration to gather the information pertaining to COTS products, it also provides services that are available only to subscribers of the portal. This is necessary for legal and economic purposes in ensuring the success of the portal; however, it also limits the input that may be obtained from users of the portal.

This thesis focuses on providing open collaboration throughout the entire process of managing dependencies and constraints. By limiting the scope to managing dependencies and constraints in software systems, economic and legal issues need not be addressed as this information is necessary in order to deploy a functional system. Therefore, it is in the best interests of an organization providing a product that as much dependency and constraint information be defined as possible since it increases the stability of the product.

\subsection{Summary}

This thesis covers several areas of rescarch, including:

1. Identifying and modeling dependencies in software systems

2. Managing dependencies and constraints in software systems

3. Using open collaboration to facilitate the management of dependencies and constraints 
This chapter described the existing research and industrial work that has been done in each of these fields. The relation of this existing work to the research carried out as part of this thesis was discussed, and how this research plans to address the issues presented by the existing work was explained.

The following chapters describe in detail the research that was carried out as part of this thesis. 


\section{Chapter 3}

\section{Modeling the Software Assembly Process}

The software engineering process defines the activities involved in developing software systems. It addresses the issues that arise throughout the lifetime of a software system by providing structure to the development process.

This chapter formalizes the application assembly process, which is the portion of the software engineering process that deals with assembling a software product using existing products, and deploying the assembled product to produce a functional system. This formalism is necessary to analyze the problem of managing constraints within a software system.

When assembling a software product from existing products, it is often the case that there exist several products that provide the same functionality. As a result, it is possible to assemble multiple instances of the same product by selecting different products.

An assembled product usually is not shipped in a state such that it may be used out of the box. It may require additional services in order to provide a functional system. The products used to provide these services are decided upon during deployment, and usually there exists more than one product that provides the same functionality. Thus, multiple instances of the same system may be deployed by selecting different products to provide the required services. 
By assembling and deploying a software product in this manner, a large number of dependencies arise between the products. Each dependency may have several constraints associated with it that must be satisfied in order for the system to function properly. These constraints must be properly managed throughout the life cycle of the software system.

Building upon the Deployment and Configuration (D\&C) Specification [7], the application assembly process is formalized by defining a set of use cases and a set of actors that are associated with the use cases. The use cases include:

- Code software products

- Select software products

- Assemble software product

- Deploy software product

- Manage constraints

A set of scenarios are presented, based upon these use cases, that describe several situations that may arise thoughout the life cycle of a software system. A set of requirements for managing constraints is then extracted from these scenarios.

This chapter is separated in the following sections:

Section 3.1: This section describes the application assembly process. The formalism of the application asscmbly process is defined using a set of use cases. Each use case is explained, as well as the actors associated with each use case.

Section 3.2: This section defines the terms dependency and constraint in the context of this research. It also describes how dependencies and constraints are discovered throughout the life cycle of a system, and how the dependencies and constraints evolve as the sytem evolves.

Section 3.3: This section presents a set of scenarios that outline several situations that may arise when assembling and deploying a software product using the application assembly process. These scenarios are then used to extract a set of requirements for the constraint management process. 
Section 3.4: This section explains the need for a formal specification for modeling software systems in order to meet the requirements of the application assembly process.

\subsection{Software Development Through Assembly}

Software systems have become increasingly large and complex. However, amid the growing demand for large scale software solutions, organizations are being forced to deliver reliable systems in less time and at a lower cost. In order to meet the challeges of producing large scale software systems, there has been a move towards assembling and integrating existing software products to create a larger product. The existing products may be produced by separate development teams in the same organization, or they may be acquired from external organizations as off-the-shelf products. The assembled product is then deployed as part of a working system. This process is referred to as the application assembly process within the context of this research.

Many software development organizations do not develop complete systems that are ready for deployment. Instead, they provide a software product that provides functionality within a narrow application domain. The reason for this is that most organizations do not possess the knowledge or skill to develop a complete software system from the ground up. Therefore, organizations have adopted the application assembly process as a means of producing software systems in less time and at a lower cost.

This section defines the application assembly process in terms of a set of actors and use cases.

\subsubsection{Application Assembly Process}

The application assembly process involves assembling a set of existing software products to create a larger software product. The assembled product can then be used as a part in a larger assembly with other products. This assembly process continues, 
creating ever larger products. At some point, a product is deployed within a particular context and becomes a systcm. In the context of this research, it is necessary to make the distinction between a software product and a system.

A software product is a piece of software that has been developed to provide functionality within a particular domain. Software products may be developed by a group of developers writing all the source code, or they may be developed by assembling and integrating a set of existing software products. Software products exist in a variety of forms, including:

- products developed internally in an organization by separate development teams

- commercial off-the-shelf (COTS) products

- open source tools or libraries

A software product is rarely self-contained; it typically requires the services of additional software products in order to function properly. In the simplest case, a software product requires the services provided by the operating system. However, in other instances, a software product may require the services of a database or an application server in order to function properly. A software product that is deployed along with the required services results in a functional system. A system includes the software product being deployed, as well as the set of software products that provide the required services.

The first step in the application assembly process is to select a set of software products, each of which implements a subset of the functionality required by the product being assembled. The chosen products must be configured, and then they are integrated by writing the necessary glue code to allow communication between the products.

The next step of the process is to deploy the assembled product. This requires selecting the software products that are required to complete the system. All of the software products must then be installed and configured to produce a functional system. 


\subsubsection{Actors and Use Cases in the Application Assembly Process}

The process of assembling an application from existing software products involves several actors, which have been defined in the context of this research. They have a relation to the actors defined in the D \& C Specification [7]; however, they have been modified in order to fit in with the requirements of the application assembly process.

The actors defined in this research, and their relation to the actors defined in the D \& C Specification, are:

- Developer: Specifies, implements and packages a software product by writing and compiling the source code. This actor is related to the Developer, Specifier, and Packager actors defined in the D \& C Specification.

- Assembler: Specifies, implements and packages a software product by assembling and integrating existing software products to produce a larger product. This actor is related to the Assembler, Specifier, and Packager actors defined in the $\mathrm{D} \& \mathrm{C}$ Specification.

- Deployer: Installs and configures all of the software products contained in a system. This actor is related to the Planner and Executor actors defined in the D \& C Specification.

- User: The end user of a software system. There is no related actor defined in the D \& C Specification.

Each of these actors is associated with a set of use cases. The use cases included in the application assembly process are (Figure 3.1):

- Code Software Product

- Select Software Products

- Assemble Software Product

- Deploy Software Product 


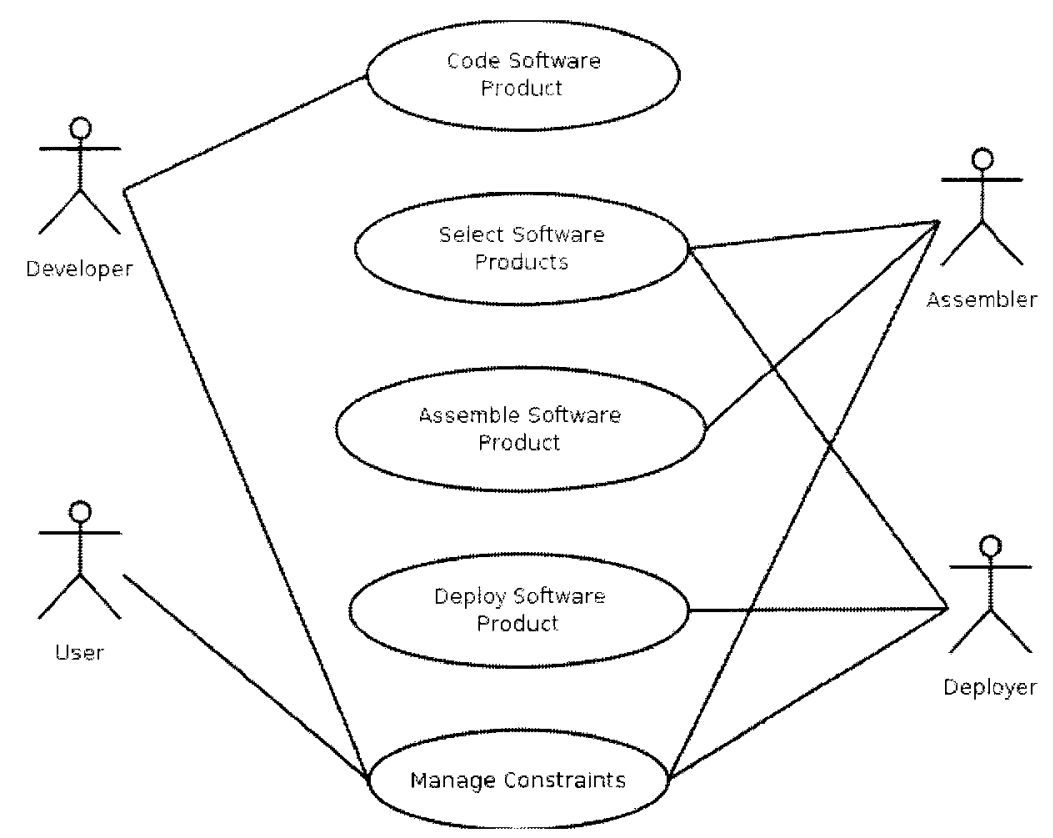

Figure 3.1: The use case diagram for the application assembly process.

- Manage Constraints

\section{Code Software Product}

In the Code Software Product use case, the Developer implements a new software product by writing the source code. The developer may code the software product from scratch or implement a portion of the product and then incorporate other software products that provide required functionality. The developer incorporates a software product by coding to a defined application programming interface (API).

For example, if a developer is implementing a product that requires data to be marshalled and unmarshalled using an XML format, it is not desirable to code this functionality from scratch. Instead, the developer may decide to use the Castor data binding framework, available from ExoLab Group [38], to handle the data serialization. The developer incorporates Castor by coding the product to use the API defined by the framework. 
Although the developer may make use of existing products, the software product that is produced is considered to be inseparable. This is because an included product can not be removed without breaking the finished product, and it cannot be replaced with a similar product without making changes directly to the source code.

The Code Software Product results in a completed software product that provides functionality in a narrow application domain, and it may be incorporated into a larger software product.

\section{Select Software Products}

In the Select Software Products use case, a set of sofware products are selected in one of two contexts:

1. The Assembler selects a set of software products that will be assembled and integrated to create a larger software product; or

2. The Deployer selects a set of software products that provide the services required by a product that is being deployed.

Although the semantics of these two contexts are quite different, the steps involved are similar as both the assembler and deployer make decisions on which software products to choose based upon a set of requirements.

During the selection process, the situation may arise where two or more products are available that provide the same functionality. This allows either the assembler or deployer to create different instances of the same software product or system, respectively.

For example, in the case of the assembler, there may exist more than one type of user interface that could be used in the product being assembled: a light-weight web based user interface that runs in a browser, or a heavy-weight Java Swing based user interface. The assembler may create two separate instances of the product by using the web interface in one instance and the Swing interface in the other. However, this decision does not change the functionality of the product being assembled; it only reflects two different instances of the same product. 
In the same regard, the deployer may be deploying a software product that requires a database. The options available to the deployer include Microsoft SQL Server [34], IBM DB2 [11] or MySQL [27]. The deployer is able to create three different instances of the same system by selecting SQL Server in one instance, DB2 in another, and MySQL in the last instance.

The Select Software Products use case results in a set of software products that may be integrated to produce a larger product, or a set of software products that may be deployed as a functional system.

\section{Assemble Software Product}

In the Assemble Software Product use case, the Assembler crcates a software product by integrating a set of software products to produce a larger software product. The integration process involves configuring the software products and writing the gluc code that is required to ailow for communication between the products. A precondition of this use case is that the software products to be integrated have been selected by the assembler.

The first step is to configure the selected software products so that they function properly within the context of the product being assembled. This involves setting any properties that affect the behaviour of a software product. For example, a data encryption/decryption product may be configured to use a particular encryption algorithm, such as the Data Encryption Standard (DES) or the Advanced Encryption Standard (AES).

The second step in the integration process is to write the glue code that is required to allow for communication between the products. In many cases, the products being integrated are written in different programming languages. Therefore, it is necessary to develop a set of language bindings to allow for the products to be interoperable. Also, the software products may use different communication protocols. This means that the assembler must write code that allows for the products to communicate using a common protocol.

The Assemble Software Product use case results in a software product that has been assembled from a set of existing products. The completed product may be 
deployed as part of a functional system, or it may be assembled with other products to produce a larger software product.

\section{Deploy Software Product}

In the Deploy Software Product use case, the Deployer deploys a software product to create a functional system. The deployment process involves installing and configuring the software products that comprise the system. A precondition of this use case is that the deployer has selected the software products that provide the services required by the product being deployed.

The first step is to install the software products onto the target hardware platform(s). Depending on the system being deployed, the products may all be installed on a single machine, or they may be installed in a distributed environment with the products running on separate machines.

The next step, after the software products have been installed, is to configure the system. This involves setting all of the appropriate configuration properties for both the individual software products and the system as a whole. For example, a database may be configured to listen for requests on a certain port, and all products that communicate with the database must be configured to send requests to the specified port.

The Deploy Software Product use case results in a functional system that is ready for use.

\section{Manage Constraints}

In the Manage Constraints use case, the Developer, Assembler, Deployer and User document and maintain all of the constraints that exist within a software system. This use case is the focus of this research, and it is discussed in detail in the following sections and the remainder of this thesis. 


\subsection{Dependencies and Constraints in Assembled Software Systems}

The application assembly process involves many independently produced software products. Assembling and deploying a software system using these products results in a large set of dependencies and constraints. Dependencies and constraints are discovered throughout the life cycle of the software system as software products are produced, deployed and used in a production environment.

This section defines the terms dependency and constraint in the context of the application assembly process. It also discusses how dependencies and constraints in a software system are discovered, as well as the effect that the evolution of a system has on the dependencies and constraints.

\subsubsection{Dependency}

A dependency is a relationship that exists between two software products. This relationship signifies that one software product requires another product in order to function properly.

In the context of this research, a dependency does not define the conditions of the relationship. For example, consider a dependency relationship between Product $A$ and ProductB. The dependency does not define whether Product $A$ requires a service provided by ProductB or whether Product A requires a particular configuration property of ProductB to be set. The dependency only shows the existence of the relationship.

Examples of dependencies are:

- If a software product uses the API defined by JDOM [19] to handle XML data, then there is a dependency between the product and the JDOM library.

- If a software product is implemented as a Java Servlet, then it requires the services provided by a Servlet container in order to run. This results in a dependency between the product and the container. 


\subsubsection{Constraint}

A constraint is any limitation or restriction that a software product has upon another software product where there exists a dependency between the two products.

Examples of constraints are:

- If a software product requires a particular version of another product, then the presence of that version of the product represents a constraint.

- If a software product requires the existence of a specific file system structure, then the presence of that file system structure represents a constraint.

- If a software product requires a particular configuration property being set in order to interoperate correctly with another product, then the value set for the configuration property represents a constraint.

Constraints are associated with a dependency, and they are used to define the nature of the dependency. For a dependency to be met, all of the constraints associated with the dependency must be satisfied. A constraint is satisfied if the restriction placed upon the system by the constraint is fulfilled.

All of the constraints within a software system must be satisfied in order for the system to function properly. If all of the constraints are not satisfied, then the system may not function as expected. Satisfying all constraints is a necessary, but not sufficient, condition for deployment.

\subsubsection{Discovery of Dependencies and Constraints}

Developing a software system using the application assembly process results in a large number of dependencies and constraints. These dependencies and constraints are discovered by all actors as they execute the use cases of the application assembly process.

The developer discovers dependencies and constraints while executing the Code Software Product use case. Each time the developer incorporates an existing product into the software product being developed, a dependency is introduced. The nature 
of this dependency is defined in terms of the constraints that must be satisfied in order for the product to function properly.

The assembler identifies dependencies and constraints while executing the Assemble Software Product use case. During the integration process, any instance where two products communicate directly, or where one product requires information provided by another product, indicates a dependency. Constraints are discovered as the assembler configures the software products and writes glue code to facilitate communication between the products. The assembler may also discover constraints within an individual software product that had not previously been identified by the developer (or assembler if the product was assembled). These new constraints may arise as a result of using the software product in a context that was not known a priori by the developer/assembler.

The deployer discovers dependencies and constraints while executing the Deploy Software Product use case. Each product that provides a service required by the product being deployed results in a dependency. Dependencies may also be identified between the set of products selected to provide the required services. Constraints are discovered as the deployer configures all of the software products in the system. Similar to the assembler, the deployer may also discover new constraints within an individual software product as it is deployed in a context that was not expected by the developer/assembler.

The user discovers constraints in a software system while using the system to perform day-to-day operations. These constraints may be identified by using the system in a manner that was not expected by the developer, assembler and/or deployer.

Dependencies and constraints may be discovered by any of the actors involved in the application assembly process. To identify all of the dependencies and constraints within a software system is a difficult task, and it is only possible with input from developers, application assemblers, deployers and users. 


\subsubsection{Evolution of Assembled Software Systems}

Software is constantly evolving over time as new features are implemented, bugs are fixed and performance is enhanced. This evolution has a dramatic effect on software systems developed using the application assembly process.

One problem posed by software evolution, in the context of the application assembly process, is that individual software products evolve independently of each other. For example, a logging library developed by one organization cvolves separately from an XML library that is developed by another organization.

In the context of the application assembly process, another problem posed by software evolution is that the software product being assembled evolves independently of the software products from which it is assembled. Assemblers may decide to evolve the product by adding new functionality; however, this has no immediate effect on the software products that are contained within the product. In terms of deployment, the products used to provide the required services to a software product evolve independently of the product itself. For example, a database evolves independently of any software product that makes use of the services offered by the database.

The result of this independent evolution amongst all of the products contained in an assembled software system is a set of constantly evolving dependencies and constraints. Managing these dependencies and constraints becomes a very difficult task due to this continuous evolution.

\subsection{Managing Constraints in Assembled Software Systems}

As discussed in the previous section, dependencies and constraints of software elements are identified by many different people including developers, application assemblers, deployers and users. These dependencies and constraints undergo constant evolution as software elements evolve independently over time. To deal with the complex task of maintaining these dependencies and constraints, it would be beneficial to have some form of a constraint management process. 
This section presents several common scenarios that arise when producing a software system using the application assembly process. The scenarios include the following situations:

- A library for maintaining user information is developed by an organization. The library makes use of third party logging library.

- The user information library implements an abstraction layer to allow for different persistence mechanisms to be used to persist the user information.

- A separate organization is assembling an on-line store product, and the user information library is selected to be included within the assembled product. Also, a persistence framework is selected to be integrated with the user library in order to allow for different databases to be used with the assembled product.

- While integrating the user information library into the on-line store product, it is discovered that the logging library is not functioning properly.

- A database is selected and the on-line store product is deploved.

- Once the on-line store product has been deployed, it is discovered that the persistence framework is sending requests to the database on the incorrect port. The configuration of the persistence framework is updated to reflect the correct port.

- A user of the on-line store discovers that the browser being used is not compatible with the deployed on-line store.

These scenarios were selected to demonstrate possible situations that may arise while carrying out the use cases defined in the application asscmbly process. Using these scenarios, a set of requirements for managing dependencies and constraints are extracted. 


\subsubsection{Constraint Management Scenarios}

Each of the scenarios described below are broken into two parts. First, a description of the scenario is given, and then the issues that arise from the scenario are discussed.

\section{Scenario: Developer-1}

Description

An organization is implementing a library for maintaining user information such as name, address and contact information. It is decided to implement logging functionality within the library. Rather than implement the logging functionality from scratch, a freely available logging library is selected and incorporated into the library.

Issues

1. The developer shall document the dependency between the user information library and the logging library. This is required for assemblers that are integrating the library into a larger application.

\section{Scenario: Developer-2}

\section{Description}

It is a requirement of the user information library described in Developer- 1 that all user information be persisted. If the application using the library expects to maintain information for a large number of users, then a database would be the optimal choice; if the application is expecting to persist the information of a small set of users, then an XML file is a better option.

Since the developer does not know a priori the context in which the library will be used, an abstraction layer is implemented that allows for any type of persistence mechanism to be integrated and used within the library.

Issues

1. The developer shall document the dependency between the library and persistence mechanism. In documenting this dependency, the developer shall explain 
which types of persistence mechanisms are supported by the library and how these mechanisms may be incorporated.

2. The solection of the persistence mechanism is left to the assembler. Depending upon which mechanism is chosen, the constraints associated with this dependency will differ.

\section{Scenario: Assembler-1}

\section{Description}

An organization is creating an on-line store that is being assembled from existing software products. The company has decided to use the library developed in Developer-1 to maintain its customers' user information.

The company is expecting a large number of customers to make use of the online store, therefore it has decided to use a relational database to persist the user information. Since the user information library uses an object oriented data model, it is necessary to map the object oriented data to the tablular structure required by the relational database. Rather than write this code from scratch, a persistence framework is selected that provides automatic object-relational data mapping. This was decided as the organization wants to assemble the product in a manner that will allow it to be used with different databases.

Issues

1. The organization that is assembling the application shall be able to vicw all of the dependencies and constraints associated with the user information library and the persistence framework.

2. The assembler shall document the dependency between the persistence framework and the database, even though the specific database hasn't been decided upon.

3. The assembler shall document any additional constraints that may be introduced by using the user information library with the persistence framework. 


\section{Scenario: Assembler-2}

Description

As the user information library is being integrated into the on-line store application discussed in Assembler-1, it is discovered that the logging functionality is not working as expected. The assembler checks all of the constraints that have been documented in relation to the dependency between the user information library and the logging library. The assembler discovers that a constraint is not properly satisfied as the user information library is loading an improper version of the logging library, which is installed in a different location on the machinc. The assembler corrects the problem by forcing the user information library to load the proper version of the logging library. Once the problem has been corrected, the logging functionality works as expected.

Issues

1. All documented constraints shall have a description that explains the restriction, as well as a method for verifying whether or not a constraint is satisfied.

\section{Scenario: Deployer-1}

\section{Description}

Once the on-line store application described in Assembler-1 has been completed, the deplover is responsible for deploving it into the company's existing environment. The database for persisting all of the data in the on-line store is selected, and it is then up to the deployer to configure the application to use the selected database. The deployer uses the dependencies and constraints that have been documented for each of the software products contained in the system to assist with the configuration process.

Issues

1. It shall be possible to search through all documented dependencies and constraints within a system. 


\section{Scenario: Deployer-2}

Description

Once the deplover has successfully completed the configuration of the persistence framework with the selected database, as described in Deployer-1, the existing test suite for the application is run. The test suite returns an error indicating that the database is not working as expected. The deployer diagnoses the problem and discovers that the database is listening for requests on a port that is different from the default port specified in the configuration of the persistence framework.

The deplover fixes the problem, and then adds the newly discovered constraint to the list of constraints already defined for using the persistence framework with the chosen database.

Issues

1. It shall be possible for anybody involved in the application assembly process to add new constraints to the system, as they are discovered.

2. Newly discovered constraints shall be distributed to the appropriate developcrs/assemblers of a software product in order to prevent people from 'reinventing the wheel' in the future.

\section{Scenario: User-1}

\section{Description}

A user is browsing the on-line store described in Assembly-1 with an older version of the Netscape [28] web browser. The user decides to purchase a product, but upon pressing the Purchase button, the web browser crashes. Further attempts lead to the same result.

The user searches through the list of defined constraints associated with the dependency between the on-line store and the Netscape browser. There exists a constraint on the required version of the web browser, but it is older than the version the user is currently using. Still, the user decides to upgrade to a newer version of the Netscape browser. 
Once the new version is installed, the user again tries to purchase the product. and this time the transaction is completed as expected. The user updates the existing constraint to reflect the need for the newer version of the Netscape web browser.

Issues

1. It shall be possible to update documented constraints that contain errors or omissions.

\subsubsection{Constraint Management Requirements}

The scenarios described in the previous section define the requirements that must be present in a constraint management process. These requirements include the ability to:

- define dependencies and constraints

- view existing constraints

- search existing constraints

- update existing constraints

- verify deployments

- distribute dependencies and constraints

- allow for open collaboration

Each of these requirements are explained in the following sections.

\section{Define Dependencies and Constraints}

It must be possible to define dependencies and constraints as they are discovered. Without the ability to define dependencies and constraints within a software system, it becomes much more difficult to effectively manage the dependencies and constraints. 


\section{View Constraints}

It must be possible to view a list of all the constraints associated with a single dependency, as well as to view each individual constraint. This allows developers. application assemblers, deplovers and users to examine the nature of a depondency and the properties of individual constraints.

\section{Search Constraints}

It must be possible to search through the list of constraints that have been defined. This allows developers, application assemblers, deployers and users to quickly determine whether certain constraints exist within the system without having to view each constraint that has been defined.

This thesis does not address the issue of how the defined constraints may be searched.

\section{Update Constraints}

If there are any errors or omissions in a defined constraint, it must be possible to update that constraint. This allows for the constraints to evolve as the system evolves.

\section{Verify Deployments}

It must be possible to verify a deployment by checking to ensure that each constraint defined within a software system is satisfied. This allows developers, application assemblers, deployers and users to determine whether the system has been set up properly.

This thesis does not address the issue of automatically verifying the constraints that have been defined within a system.

\section{Distribute Dependencies and Constraints}

It must be possible to distribute all dependency and constraint information defined for a software element. This will allow for application assemblers to easily import 
existing dependencies and constraints for software elements that are being integrated into an application.

Any additional constraints that are defined by application assemblers, deployers and users upon a software element should be reflected back to the developers of the software product.

\section{Open Collaboration}

It must be possible for developers, application asscmblers, deployers and users to perform the above mentioned actions. Since dependencies and constraints may be discovered by any one of these groups of people, it is important to provide a mechanism that promotes open collaboration between all involved with the software system.

\subsection{Need for a Formal Specification}

The constraint management process discussed in the previous section involves numerous actions that must be able to be performed in order to properly manage all of the dependencies and constraints in a software system. These requirements present the need for a formal specification to provide a structure for modeling dependencies between software products.

The formal specification should provide a method for defining the structure of a software system by specifying the software products contained within the system and the dependencies between these products. It should also allow for the hierarchical decomposition of a software product. This is necessary as the product may be comprised of a set of assembled software products.

Once the structure of a software system has been formally modeled, the process of managing dependencies and constraints is simplified, as the actions described in the previous section may be performed. 


\subsection{Summary}

Software systems are becoming increasingly large and complex. In order to facilitate the development of these large scale systems, the method in which software is developed has evolved. It is becoming more common for applications to be assembled from existing software products. However, this process of assembling applications leads to a large network of dependencies and constraints between the software products.

This chapter presented a use case model of the application assembly process and discussed how dependencies and constraints are discovered and how they evolve over time. A process for managing these dependencies and constraints was described, and the requirements for this process were introduced. Finally, the need for a formal model to facilitate the constraint management process was discussed.

The following chapter describes this formal specification that was developed as part of this research. 


\section{Chapter 4}

\section{Modeling Assembled Software Systems}

The preceding chapter defined a formalization of the application assembly process. Developing a software system using the application assembly process results in a large number of dependencies and constraints, which are discovered by all actors involved with the system. The management of these dependencies and constraints presented several issues including the ability to:

- define dependencies and constraints

- view existing constraints

- search existing constraints

- update existing constraints

- automatically verify deployments

- distribute dependencies and constraints

- provide open collaboration

Addressing these issues requires a process, as well as a set of tools to support the process. The process and tools should be based upon a formal specification for modelling an assembled software system. This chapter presents a formal specification for 
modelling the products, dependencies and constraints within a software system that has been developed using the application assembly process. This may be used to meet the requirements of managing dependencies and constraints that were discussed in Section 3.3.2 of Chapter 3.

The formal specification defined in this chapter allows for the software products within a system to be modeled as components, where a component represents any software element. Software elements include, but are not limited to, libraries, executables and configuration files. There are three types of components that may be defined within a software system:

- Concrete: This reprensents a single, non-decomposable software element that implements a particular function within the system

- Virtual: This represents a software element that defines a particular function within the system, but it does not provide an implementation

- Composite: This represents a software element that may be decomposed into a set of software elements

A software system is modeled as a configuration within the formal specification. A configuration consists of a set of assemblies that represent the hierarchical decomposition of the system. An assembly contains a set of components and a set of dependencies, where each dependency represents a relationship between two components. Each assembly also consists of a set of constraints, with each constraint associated with a dependency.

When modeling a software system, virtual components are used to represent the selection points within the system where multiple software products are available that provide the same functionality. A configuration containing virtual components may be specialized to reflect the decisions made when selecting the products to be used within the system. Creating a specialization involves replacing a virtual component with either a concrete or composite component.

Each of the elements defined within the formal specification are described in the following sections. 
Section 4.1: This section defines the component element of the formal specification. There are three types of components defined: concrete, virtual, and composite. A graphical representation of each component type is also presented.

Section 4.2: This section defines the dependency element of the formal specification. A dependency represents a relationship between two components. A graphical representation for dependencies is also presented.

Section 4.3: This section defines the constraint element of the formal specification. A constraint represents any restriction that exists within a software system.

Section 4.4: This section defines the assembly element of the formal specification. An assembly consists of a set of components, a set of dependencies, a set of constraints and a function that maps each constraint to a dependency. A graphical representation for an assembly is also presented.

Section 4.5: This section defines the configuration element of the formal specification. A configuration consists of a set of assemblies and a function that maps each composite component contained in the configuration to the decomposition assembly of the component. A graphical representation for a configuration is also presented.

Section 4.6: This section defines how to create a specialization within the formal specification. A configuration is specialized by replacing a virtual component with either a concrete or composite component.

Section 4.7: This section defines the specialization scheme element of the formal specification. A specialization scheme consists of all of the configurations that have been defined for a software system. This includes all of the specializations that have been defined for a system.

\subsection{Components}

\subsubsection{Description}

The component element is a primitive building block in the formal specification. A component is any software element that exists within a system, where a software element may be a tool, framework, library, configuration file, or any other software 
related item required by the system.

A software product may be decomposed into several components. For example, a software product may consist of a binary cxecutable file, several library files (ie. DLL files or JAR files), and a configuration file. It is up to the modeler to decide whether a software product is represented as a single component or decomposed into a set of components.

Thore are three types of components:

- Concrete

- Virtual

- Composite

A concrete component implements services without being divisible into subcomponents. Although from a modeling perspective a concrete component is indivisible, it may have a complex structure. What constitutes a concrete component is up to the modeler: a set of files, modules, and/or objects may be viewed as a single concrete component or as multiple concrete components. In the context of the application assembly process, a concrete component would be used for any software product that has been decided upon by either the Assembler or Deployer to be incorporated into the system.

A virtual component defines the services offered, but does not provide an implementation. It serves as an abstraction layer that allows for any component that provides the required services to be substituted into the system. In relation to the application assembly process, a virtual component would be used to represent a subset of functionality required by the system, where the product offering that functionality has not yet been decided upon.

A composite component defines the services offered by a set of components that can be combined and treated as a single component. Composite components are used to group related components that interact to provide a subset of the functionality within a system. In terms of the application assembly process, a composite component may be used to represent a software product that is composed of more than one component. 


\subsubsection{Definition}

Components are primitive elements that cannot be subdivided into smaller elements. There are three types of components.

The set of all concrete components is represented as $\mathbb{P}$.

The set of all virtual components is represented as $\mathbb{V}$.

The set of all composite components is represented as $\mathbb{Z}$.

The set of all components is represented as $\mathbb{C}$, such that

$$
\mathbb{C}=\mathbb{V} \cup \mathbb{P} \cup \mathbb{Z} \quad \text { where } \mathbb{V}, \mathbb{P} \text {, and } \mathbb{Z} \text { are disjoint. }
$$

\subsubsection{Graphical Notation}

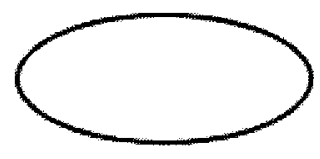

Virtual

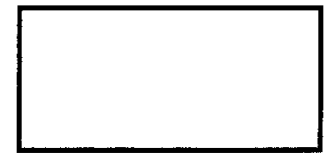

Concrete

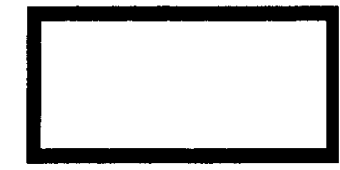

Composite

Figure 4.1: Graphical representation of each component type.

The graphical representation of each type of component is illustrated in Figure 4.1. A virtual component is depicted as an oval, a concrete component as a rectangle with a thin border, and a composite component as a rectangle with a thick border.

\subsection{Dependencies}

\subsubsection{Description}

A component may require another component in order to function properly. This requirement is called a dependency. A dependency is a relationship that exists between two components within a system. A dependency is used to show the existence of a 
relationship, however, it does not define the restrictions that are placed upon the system by the dependency.

The dependencies of the formal specification represent the dependencies discussed in Chapter 3. For example, if there is a dependency identified between two software products while assembling a new software product, then there must be a corresponding dependency defined between the two components representing the software products within the system model.

Dependencies are used to express a relationship between two components within a system; however, a dependency does not infer any details regarding the relationship. Thus, dependencies are non-directional within the formal specification.

\subsubsection{Definition}

A dependency is expressed as an unordered pair of components. A set of components may have a set of dependencies associated with it, where each dependency consists of two unique components from the set of components.

Definition 1 (Dependency Set). Given a finite set of components, $C$, a dependency set, $\boldsymbol{D}$, of $\boldsymbol{C}$ is a set of unordered pairs, $\left(c_{1}, c_{2}\right)$, such that $c_{1}, c_{2} \in \boldsymbol{C}$ and $c_{1} \neq c_{2}$.

\subsubsection{Graphical Notation}

A dependency is depicted as a solid line that connects both components involved in the dependency relationship (Figure 4.2). Figure 4.2 illustrates the following set of dependencies:

$$
D=\{(\text { Component } A, \text { ComponentB }),(\text { Component } A, \text { ComponentC })\}
$$




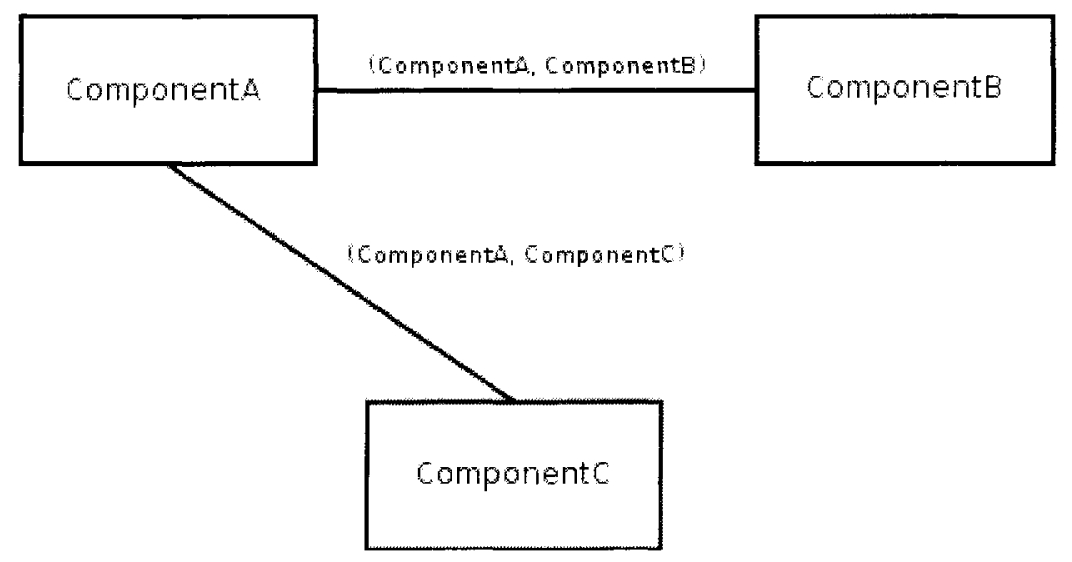

Figure 4.2: The graphical representation of a dependency.

\subsection{Constraints}

\subsubsection{Description}

A dependency is used to define a relationship between two components. The restrictions imposed upon the system by the dependency are defined as constraints that are associated with the dependency. A constraint is a condition that is added to a dependency that must evaluate to true in order for the dependency to be satisfied. A dependency can have any number of constraints associated with it.

The constraints of the formal specification represent the constraints defined in Chapter 3. For example, if a constraint has been identified while assembling and deploying a software product, there there must be a corresponding constraint defined within the system model.

\subsubsection{Definition}

Constraints are primitive elements that cannot be subdivided into smaller elements.

The set of all constraints is represented as $\mathbb{Q}$. 


\subsubsection{Graphical Notation}

There is no graphical representation for constraints.

\subsection{Assemblies}

\subsubsection{Description}

A set of related components may be grouped together to form an assembly. An assembly consists of a set of components and all of the dependencies that exist amongst the components. Any constraints that have been associated with the dependencies are also included in the assembly.

Assemblies are used to hierarchically decompose components. An assembler may create an assembly to decompose an individual software product into a set of components, or a set of related software products may be grouped together to create an assembly.

\subsubsection{Definition}

An assembly is a set of components, a dependency set on the components, a set of constraints, and a function that maps each constraint to a dependency.

Definition 2 (Assembly). An assembly, $a$, is a four-tuple

$$
a=(\boldsymbol{C}, \boldsymbol{D}, \boldsymbol{Q}, m)
$$

where:

1. $C \subset \mathbb{C}$ is a finite set of components

2. $D$ is a dependency set of $C$

3. $Q \subset \mathbb{Q}$ is a finite set of constraints 
4. $m$ is a function that maps each constraint in $\boldsymbol{Q}$ to a dependency in $\boldsymbol{D}$, such that $m(q)=d$, where $q \in \boldsymbol{Q}$, and $d \in \boldsymbol{D}$

The set of all assemblies is represented as $\mathbb{A}$.

Given a set of assemblies, it is possible to define the set of all concrete, virtual and composite components that exist within the set of assemblies. It is also possible to define the set of all constraints that exist within a set of assemblies.

Definition 3 (CONCRETE $(\boldsymbol{A})$ ). Given a set of assemblies, $\boldsymbol{A} \subset \mathbb{A}$, CONCRETE $(\boldsymbol{A})$ is the set of all concrete components in $\boldsymbol{A}$, and is defined as

$$
\operatorname{COncrete}(\boldsymbol{A})=\bigcup_{a \in \boldsymbol{A}} \boldsymbol{P}_{\boldsymbol{a}}
$$

where

$$
\begin{aligned}
& a=\left(\boldsymbol{C}_{\boldsymbol{a}}, \boldsymbol{D}_{\boldsymbol{a}}, \boldsymbol{Q}_{\boldsymbol{a}}, m_{a}\right) \\
& \boldsymbol{C}_{\boldsymbol{a}}=\boldsymbol{P}_{\boldsymbol{a}} \cup \boldsymbol{V}_{\boldsymbol{a}} \cup \boldsymbol{Z}_{\boldsymbol{a}}
\end{aligned}
$$

9

Definition 4 (VIRTUAL $(\boldsymbol{A})$ ). Given a set of assemblies, $\boldsymbol{A} \subset \mathbb{A}$, $\operatorname{VIRTUAL}(\boldsymbol{A})$ is the set of all virtual components in $\boldsymbol{A}$, and is defined as

$$
\operatorname{ViRTUAL}(\boldsymbol{A})=\bigcup_{a \in \boldsymbol{A}} \boldsymbol{V}_{\boldsymbol{a}}
$$

where

$$
\begin{aligned}
& a=\left(\boldsymbol{C}_{a}, \boldsymbol{D}_{\boldsymbol{a}}, \boldsymbol{Q}_{\boldsymbol{a}}, m_{a}\right) \\
& \boldsymbol{C}_{\boldsymbol{a}}=\boldsymbol{P}_{\boldsymbol{a}} \cup \boldsymbol{V}_{\boldsymbol{a}} \cup \boldsymbol{Z}_{a}
\end{aligned}
$$


Definition 5 (COMPOSITE $(\boldsymbol{A})$ ). Given a set of assemblies, $\boldsymbol{A} \subset \mathbb{A}$.

$\operatorname{COMPOSITE}(\boldsymbol{A})$ is the set of all composite components in $\boldsymbol{A}$, and is defined as

$$
\operatorname{COMPOSITE}(\boldsymbol{A})=\bigcup_{a \in \boldsymbol{A}} Z_{a}
$$

where

$$
\begin{aligned}
& a=\left(\boldsymbol{C}_{\boldsymbol{a}}, \boldsymbol{D}_{\boldsymbol{a}}, \boldsymbol{Q}_{\boldsymbol{a}}, m_{a}\right) \\
& \boldsymbol{C}_{\boldsymbol{a}}=\boldsymbol{P}_{\boldsymbol{a}} \cup \boldsymbol{V}_{\boldsymbol{a}} \cup \boldsymbol{Z}_{\boldsymbol{a}}
\end{aligned}
$$

9

Definition 6 (CONSTRAINT $(\boldsymbol{A})$ ). Given a set of assemblies, $\boldsymbol{A} \subset \mathbb{A}$, $\operatorname{CONSTRAINT}(\boldsymbol{A})$ is the set of all constraints in $\boldsymbol{A}$, and is defined as

$$
\operatorname{CONSTRAINT}(\boldsymbol{A})=\bigcup_{\boldsymbol{a} \in \boldsymbol{A}} \boldsymbol{Q}_{\boldsymbol{a}}
$$

where

$$
a=\left(C_{a}, D_{a}, Q_{a}, m_{a}\right)
$$

\section{9}

\subsubsection{Graphical Notation}

The graphical representation of an assembly is shown in Figure 4.3. An assembly is depicted as a container consisting of all components within the assembly and the dependencies that exist between the components. There is no graphical representation for a constraint.

\subsection{Configurations}

\subsubsection{Description}

A configuration represents the hierarchical decomposition of a system through the use of composite components. A configuration consists of a set of assemblies that 


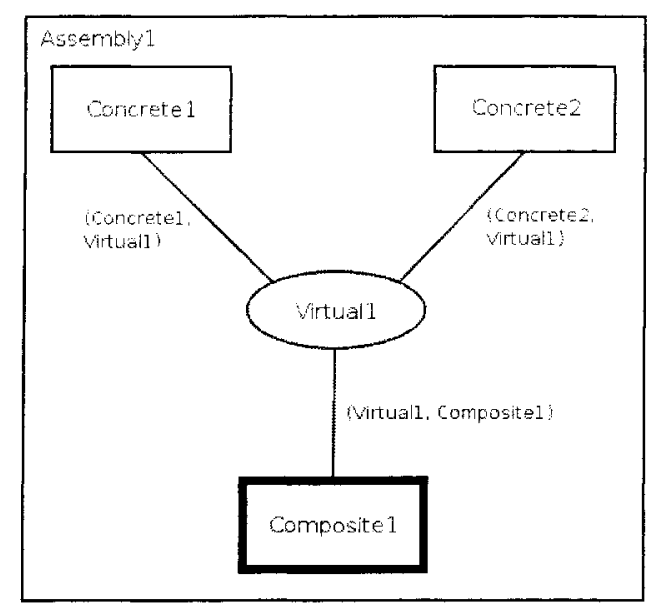

Figure 4.3: Graphical representation of an assembly.

contain all of the components, dependencies and constraints that have been defined within a software system. It also consists of a function that maps each composite component within the configuration to the assembly containing the decomposition of the composite component.

A configuration must be valid in order for a system to be properly modeled. A configuration is valid if and only if:

- Each component within the configuration exists in a single assembly. That is, if a component, Comp1, exists in the assembly Assembly1, then Comp1 cannot exist in any other assembly within the configuration.

- The configuration has a top level assembly that represents the root of the system's hierarchical decomposition. Every other assembly in the configuration is a decomposition assembly of a composite component contained within the configuration.

- Each composite component in the configuration has a unique decomposition assembly within the configuration.

A valid configuration that contains no virtual components represents a deployable system. 


\subsubsection{Definition}

A configuration is a set of assemblies and a function that maps each composite component to the assembly that represents the dccomposition of the composite component.

Definition 7 (Configuration). A configuration, $t$, is a tuple

$$
t=(\boldsymbol{A}, f)
$$

where

1. $\boldsymbol{A} \subset \mathbb{A}$ is a finite set of assemblies

2. $f$ is a function that maps each composite component in $\operatorname{COMPOSITE}(\boldsymbol{A})$ to a unique assembly in $\boldsymbol{A}$, such that for every $z_{i}, z_{j} \in \operatorname{COMPOSITE}(\boldsymbol{A})$,

$$
f\left(z_{i}\right)=f\left(z_{j}\right) \text { if and only if } z_{i}=z_{j}
$$

The set of all configurations is represented as $\mathbb{T}$.

A configuration may be represented as a directed graph where the vertices of the graph are the assemblies of the configuration, and the edges of the graph are the mappings from each composite component to the corresponding decomposition assembly.

Definition 8 (Configuration Graph). A configuration graph, $g=(\boldsymbol{V}, \boldsymbol{E})$, of the configuration $t=(\boldsymbol{A}, f)$ is a directed graph where

1. The set of vertices, $\boldsymbol{V}$, of $g$ is equal to the set of assemblies of $t$. Thus,

$$
V=A
$$


2. $\boldsymbol{E}$ is the set of edges such that $\left(a_{1}, a_{2}\right)$ is an edge in $\boldsymbol{E}$. where $a_{1}, a_{2} \in \boldsymbol{A}$ and $a_{1}=\left(\boldsymbol{C}_{\mathbf{1}}, \boldsymbol{D}_{\mathbf{1}}, \boldsymbol{Q}_{\mathbf{1}}, m_{1}\right)$, if and only if there exists a component, $c \in \boldsymbol{C}_{\mathbf{1}}$ that is mapped to the assembly $a_{2}$ by the function $f$. That is, given

$$
a_{1}=\left(\boldsymbol{C}_{\mathbf{1}}, \boldsymbol{D}_{\mathbf{1}}, \boldsymbol{Q}_{\mathbf{1}}, m_{1}\right), a_{2} \in \boldsymbol{A}
$$

then

$$
\left(a_{1}, a_{2}\right) \in \boldsymbol{E} \text { iff } \exists c \in \boldsymbol{C}_{1} \text { such that } f(c)=a_{2}
$$

In order for a configuration to be valid, each component within the configuration must exist in exactly one assembly. Although it is possible to use the same physical software product for more than one component within a system, from a modeling perspective, the components are unique as they are used in different contexts within the system.

As well, the configuration graph must be connected, for the configuration to be valid, so that each assembly is mapped to a composite component, except for a single, top level assembly. This top level assembly represents the root of the hierarchical decomposition of the system, and it is not a decomposition assembly for any composite component. Finally, the configuration graph must be acyclic so that a composite component cannot be a subcomponent of itself.

Definition 9 (Valid Configuration). Given a configuration, $t=(\boldsymbol{A}, f)$, where

$$
\begin{aligned}
& \boldsymbol{A}=a_{1}, a_{2} \ldots, a_{n} \\
& a_{i}=\left(\boldsymbol{C}_{\boldsymbol{i}}, \boldsymbol{D}_{\boldsymbol{i}}, \boldsymbol{Q}_{\boldsymbol{i}}, m_{i}\right)
\end{aligned}
$$

then $t$ is a valid configuration if and only if

1. $C_{1}, C_{2}, \ldots, C_{n}$ are disjoint

2. The configuration graph of $t$ is connected

3. The configuration graph of $t$ is acyclic 
A property of the configuration graph of a valid configuration is that it is a tree rooted at the only assembly that is not mapped to by a composite component. In order to prove this property, it is necessary to prove several other properties of a valid configuration.

First, it will be shown that a valid configuration must contain at least one assembly that is not mapped to by a composite component contained within the configuration.

Lemma 1. Given a valid configuration, $t=(\boldsymbol{A}, f)$, there exists an assembly, $a \in \boldsymbol{A}$, that is not mapped to by a composite component in $\operatorname{COMPOSITE}(\boldsymbol{A})$. That is,

$$
\exists a \in \boldsymbol{A} \text { such that } \forall z \in \operatorname{COMPOSITE}(\boldsymbol{A}) f(z) \neq a
$$

This implies that the configuration graph, $g=(\boldsymbol{V}, \boldsymbol{E})$, of $t$ contains a vertex, $v \in \boldsymbol{V}$, that has no incoming edges. That is,

$$
\exists \mathrm{v} \in \boldsymbol{V}, \text { degree }_{\text {in }}(v)=0
$$

where degree $_{i n}(v)$ is the number of incoming edges of the vertex $v$.

Proof: This will be proven by contradiction.

Assume there does not exist an assembly, $a \in \boldsymbol{A}$, that is not mapped to by a composite component in COMPOSITE $(\boldsymbol{A})$. That is,

$$
\exists \mathrm{z} \in \operatorname{COMPOSITE}(\boldsymbol{A}) \text { such that } f(z)=a
$$

This implies that the configuration graph, $g$, of $t$ does not contain a vertex, $v \in \boldsymbol{V}$, that has no incoming edges. That is,

$$
\nexists \mathrm{v} \in \boldsymbol{V} \text { such that degree }{ }_{\text {in }}(v)=0
$$

Since there is no vertex in $g$ with zero incoming edges, there must exist a cycle in $g$. By definition, the configuration graph of a valid configuration must be acyclic, thus, this contradicts the fact that $t$ is a valid configuration. 
Therefore, if $t$ is a valid configuration. $g$ must contain at least one vertex with zero incoming edges, and this implies that $t$ contains at least one assembly that is not mapped to by a composite component contained within the configuration. $Q E D$

Next, it will be shown that there is at most one assembly in a valid configuration that is not mapped to by a composite component contained within the configuration.

Lemma 2. Given a valid configuration, $t=(\boldsymbol{A}, f)$, if the assemblies, $a_{1}, a_{2} \in \boldsymbol{A}$, are not mapped to by a composite component in COMPOSITE $(\boldsymbol{A})$, then $a_{1}$ and $a_{2}$ must be the same assembly. That is, if $\exists a_{1}, a_{2} \in \boldsymbol{A}$ such that

$$
\forall z \in \operatorname{COMPOSITE}(\boldsymbol{A}), f(z) \neq a_{1} \text { and } f(z) \neq a_{2}
$$

then

$$
a_{1}=a_{2}
$$

Proof: This will be proven by contradiction.

Assume that there are two assemblies, $a_{1}, a_{2} \in \boldsymbol{A}$, that are not mapped to by composite components in $\operatorname{COMPOSITE}(\boldsymbol{A})$, and $a_{1}$ and $a_{2}$ are not the same assembly. That is, if $\exists a_{1}, a_{2} \in \boldsymbol{A}$ such that

$$
\forall z \in \operatorname{COMPOSITE}(\boldsymbol{A}), f(z) \neq a_{1} \text { and } f(z) \neq a_{2}
$$

then

$$
a_{1} \neq a_{2}
$$

It follows that the configuration graph, $g$, of $t$ contains at least two vertices, $v_{1}, v_{2} \in \boldsymbol{V}$. that have zero incoming edges. That is,

$$
\exists v_{1}, v_{2} \in \boldsymbol{V} \text { such that } \operatorname{degre\epsilon }_{i n}\left(v_{1}\right)=\operatorname{degre\epsilon }_{\text {in }}\left(v_{2}\right)=0
$$

By Definition 7, each composite component in COMPOSITE $(\boldsymbol{A})$ maps to a unique assembly, thus the configuration graph, g, of $t$ must be disconnected. By Definition 8, the configuration graph of a valid configuration must be connected, thus, this condradicts the fact that $t$ is a valid configuration. 
Therefore, if $t$ is a valid configuration, $g$ must contain at most one vertex with zero incoming edges, and this implies that $t$ contains at most one assembly that is not mapped to by a composite component contained within the configuration.

$Q E D$

Using the above properties, it is possible to prove that the configuration graph of a valid configuration is a tree rooted at the only assembly in the configuration that is not mapped to by a composite component contained in the configuration. This property is relevant because it demonstrates that the hierarchical decomposition of a software system may be represented as a tree.

This is proven in the following theorem by combining the results of the above lemmas.

Theorem 1. The configuration graph, $g=(\boldsymbol{V}, \boldsymbol{E})$, of a valid configuration, $t=$ $(\boldsymbol{A}, f)$, is a tree that is rooted at the only vertex, $v \in \boldsymbol{V}$, that has $\operatorname{degree}_{i n}(v)=0$.

Proof: This will be proven by showing that the configuration graph of $t$ :

1. Has a single root node;

2. Is connected; and

3. Is acyclic

By Lemma 1, the configuration graph, $g$, of the valid configuration, $t$, contains at least one vertex, $v_{R} \in \boldsymbol{V}$, such that degree $_{i n}\left(v_{R}\right)=0$.

By Lemma 2, the configuration graph, $g$, of the valid configuration, $t$, contains at most one vertex, $v_{R} \in \boldsymbol{V}$, such that degree ${ }_{i n}\left(v_{R}\right)=0$.

By combining the results of Lemma 1 and Lemma 2, it can be concluded that the configuration graph, $g$, of the valid configuration, $t$, has exactly one vertex, $v_{R} \in \boldsymbol{V}$, such that degree $e_{i n}\left(v_{R}\right)=0$. This vertex is the root of the tree.

In $t$, each composite component, $z \in \operatorname{COMPOSITE}(\boldsymbol{A})$, exists in a single assembly (see Definition 9 ). and $z$ is mapped to a unique assembly by $f$ (see Definition 7 ). 
The configuration graph, $g$ of $t$ contains an edge, $e \in \boldsymbol{E}$. for each mapping of a composite component to an assembly (see Definition 8). As a result, the number of edges in $\boldsymbol{E}$ is equal to the number of composite components in $\operatorname{COMPOSITE}(\boldsymbol{A})$. Thus,

$$
|\boldsymbol{E}|=|\operatorname{COMPOSITE}(\boldsymbol{A})|
$$

The configuration graph, $g$, of $t$ contains a vertex, $v \in \boldsymbol{V}$, for each assembly that is mapped to by a composite component, plus the vertex, $v_{R}$, for the assembly that does not contain any incoming edges. As a result, the number of vertices in $\boldsymbol{V}$ is equal to the number of composite components in $\operatorname{COMPOSITE}(\boldsymbol{A})$ plus one. Thus,

$$
|\boldsymbol{V}|=|\operatorname{COMpOSiTE}(\boldsymbol{A})|+1
$$

It follows that

$$
|\boldsymbol{V}|=|\boldsymbol{E}|-1
$$

Since the configuration graph, $g$, of the valid configuration, $t$, is an acyclic, connected graph with $|\boldsymbol{V}|=|\boldsymbol{E}|-1$, it is, by definition, a tree rooted at the vertex $v_{R}$, which is the only vertex in $\boldsymbol{V}$ with zero incoming edges.

$Q E D$

A configuration that contains no virtual components is called a deployable configuration. A deployable configuration contains all of the components that have been selected for a particular instance of the system, the dependencies between the components, and all of the constraints that exist within this instance of the system. A deployable configuration should represent a functional system that may be deployed.

Definition 10 (Deployable Configuration). A configuration, $t=(\boldsymbol{A}, f)$, is a deployable configuration if and only if

1. $t$ is a valid configuration 
2. $t$ contains no virtual components. That is,

$$
|\operatorname{VIRTUAL}(\boldsymbol{A})|=0
$$

\subsubsection{Graphical Notation}

The graphical representation of a valid configuration is shown in Figure 4.4. A con-

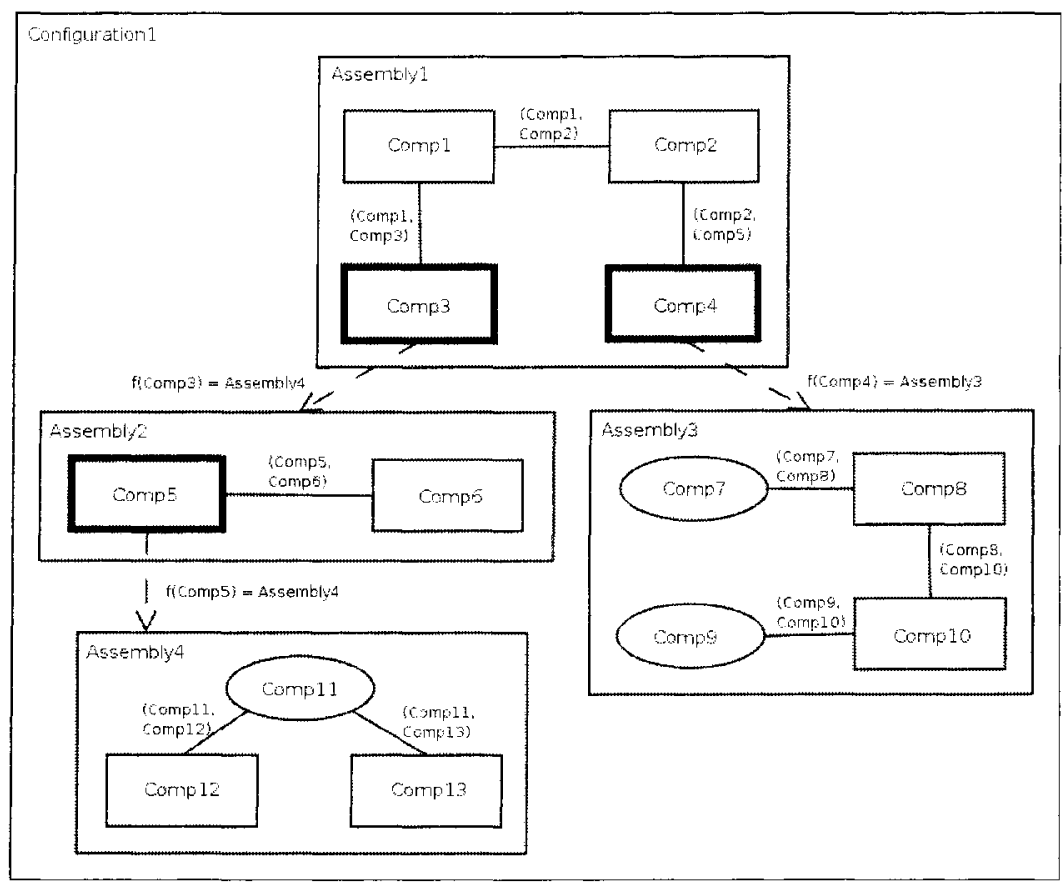

Figure 4.4: Graphical representation of a configuration.

figuration is depicted as a container with all assemblies of the configuration enclosed within the container. The mapping from each composite component to the corresponding decomposition assembly is illustrated using a dashed arrow (Figure 4.4). Figure 4.5 shows the configuration graph of the configuration presented in Figure 4.4. Since the configuration is valid, the configuration graph is also a configuration tree, rooted at Assembly1. 


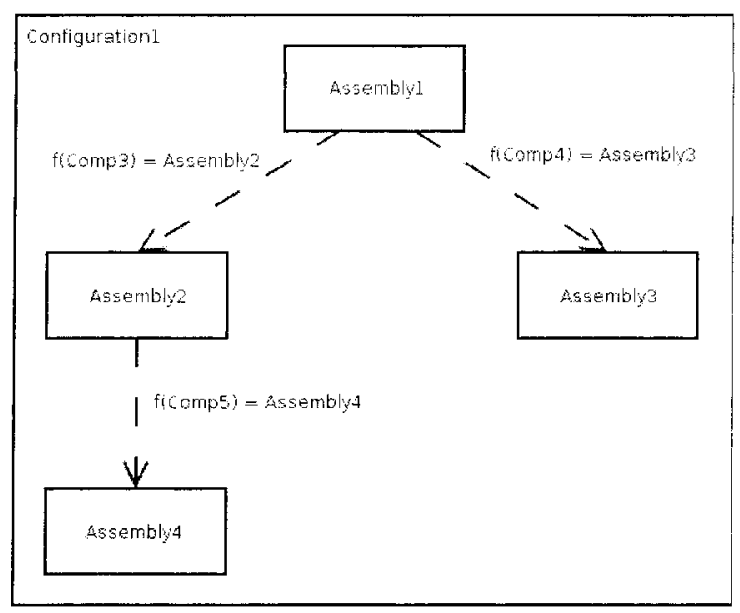

Figure 4.5: Graphical representation of a configuration graph.

\subsection{Specializations}

\subsubsection{Description}

For a functional system to be deployed, it is necessary to have a deployable configuration that is a valid configuration containing no virtual components. Any configuration that contains virtual components may not be deployed, as virtual components have no implementation. In order to create a deployable configuration from a configuration containing virtual components, a series of specializations must be created. A configuration, $A$, may be specialized by replacing a virtual component with either a concrete or composite component. This results in a new configuration, $B$, which is a specialization of $A$. When replacing a virtual component with a concrete or composite component, all of the dependency and constraint information pertaining to the virtual component must be preserved in the replacement component, such that:

- All of the dependency relationships that contain the virtual component must be updated to include the replacement component;

- All of the constraints associated with dependency relationships that contain the virtual component must become associated with the updated dependencies. 
The remaining components, dependencies, and constraints in the original configuration also exist in the new configuration.

A specialization may contain additional constraints that have been discovered as a result of replacing a virtual component with either a concrete or composite component.

Configurations are specialized by assemblers and deployers as they select the software products to be used in a system. Every time that a product is decided upon, the configuration that existed prior to the selection of that product is specialized to create a new configuration that reflects the decision made.

During the product selection process, there are three possible scenarios where a configuration may be specialized to create a new configuration:

1. A virtual component in configuration $A$ is replaced with a new concrete component in configuration $B$, where the new component did not previously exist in $A$.

2. A virtual component in configuration $A$ is replaced with a new composite component in configuration $B$, where the new component did not previously exist in $A$.

3. A virtual component in configuration $A$ is replaced with a concrete or composite component in configuration $B$, where the replacement component existed in the same assembly within $A$ as the virtual component that was replaced.

An example of each scenario is provided below.

\section{Virtual Component Replaced with New Concrete Component}

To demonstrate replacing a virtual component with a new concrete component, the configuration Configuration 1 is specialized to produce the configuration Configuration? (Figure 4.6). This is accomplished by replacing the virtual component Comp6 in Assembly2 of Configuration1 with the concrete component Comp12 in Assembly4 of Configuration:. The rest of the components in Assembly2 also exist in Assembly4. 

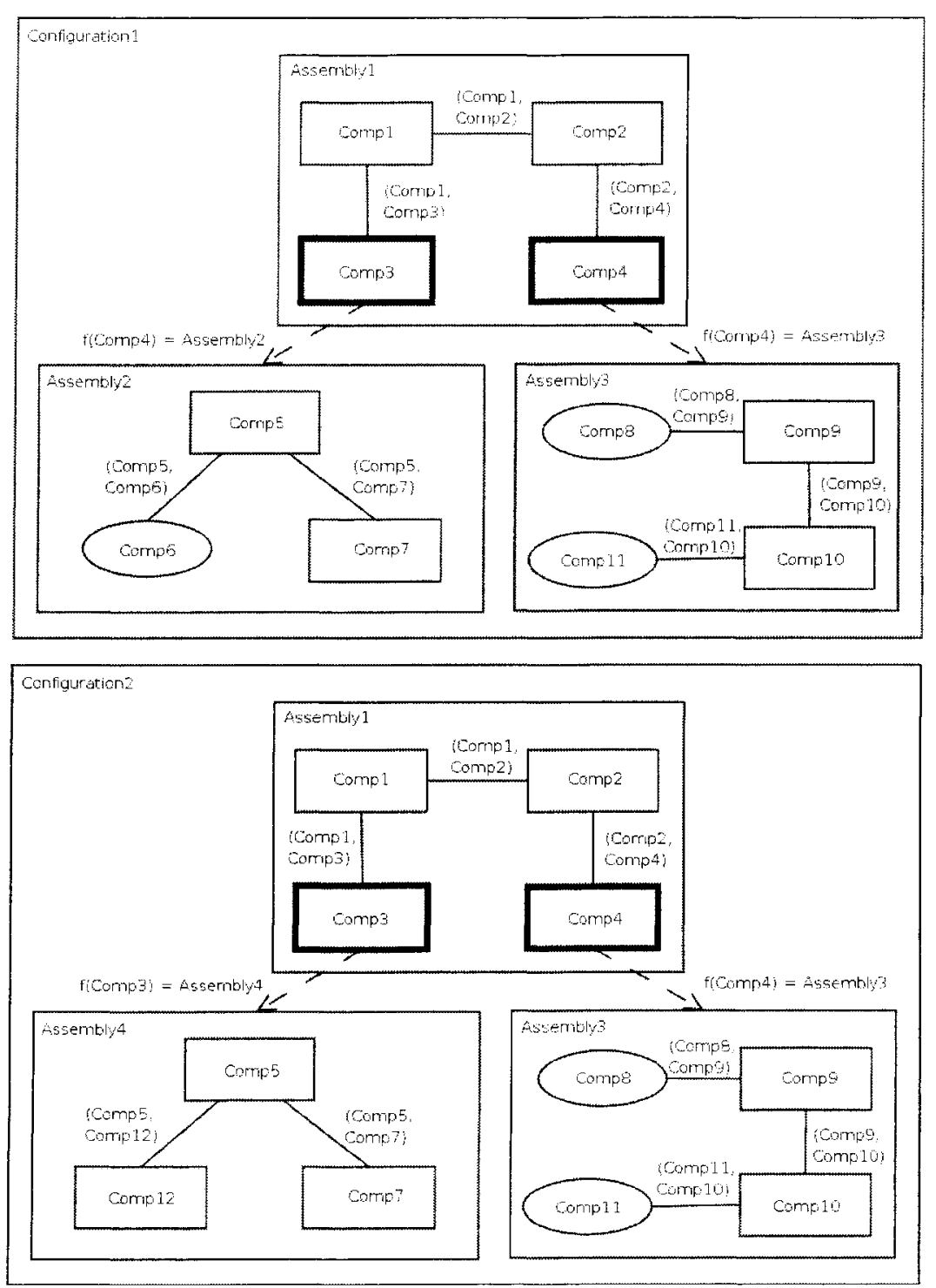

Figure 4.6: Specialization of a configuration by replacing a virtual component with a concrete component. 
The dependency (Comp5, Comp6) in Assembly2 of Configuration1 is replaced with the dependency (Comp5, Comp12) in Assembly4 of Configuration:. All other dependencies in Assembly? also exist in Assembly4.

All of the constraints that are associated with the dependency (Comp5. Comp6) in Assembly? become associated with the dependency (Comp5, Comp12) in Assembly4. All constraints that are associated with the other dependencies in Assembly2 are associated with the same dependencies in Assembly4.

The rest of the assemblies in the original configuration, Configuration1, also exist in the new configuration, Configurationt.

\section{Virtual Component Replaced with New Composite Component}

To demonstrate replacing a virtual component with a new composite component, the configuration Configuration1 is specialized to produce the configuration Configuration3 (Figure 4.7). This is accomplished by replacing the virtual component Comp6 in Assembly2 of Configuration 1 with the composite component Comp13 in Assembly5 of Configuration3. The rest of the components in Assembly2 also exist in Assembly5.

The dependency (Comp5, Comp6) in Assembly2 of Configuration1 is replaced with the dependency (Comp5, Comp13) in Assembly5 of Configuration3. All other dependencies in Assembly2 also exist in Assembly5.

All of the constraints that are associated with the dependency (Comp5, Comp6) in Assembly2 become associated with the dependency (Comp5, Comp13) in Assembly5. All constraints that are associated with the other dependencies in Assembly 2 are associated with the same dependencies in Assembly5.

The rest of the assemblies in the original configuration, Configuration1, also exist in the new configuration, Configuration?. The new configuration also contains the decomposition assembly, Assembly6, of the new composite component, Comp 13. 

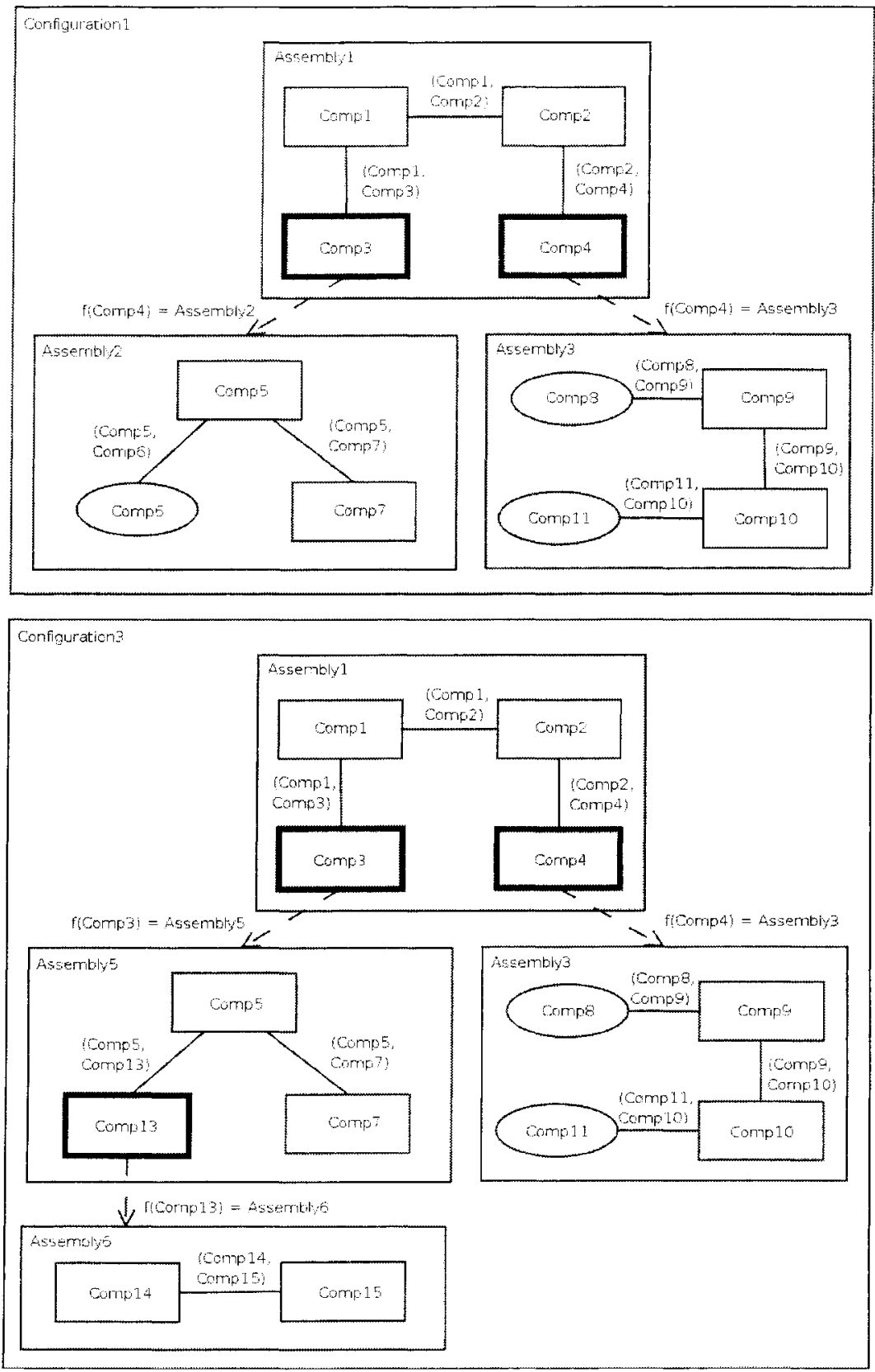

Figure 4.7: Specialization of a configuration by replacing a virtual component with a composite component. 

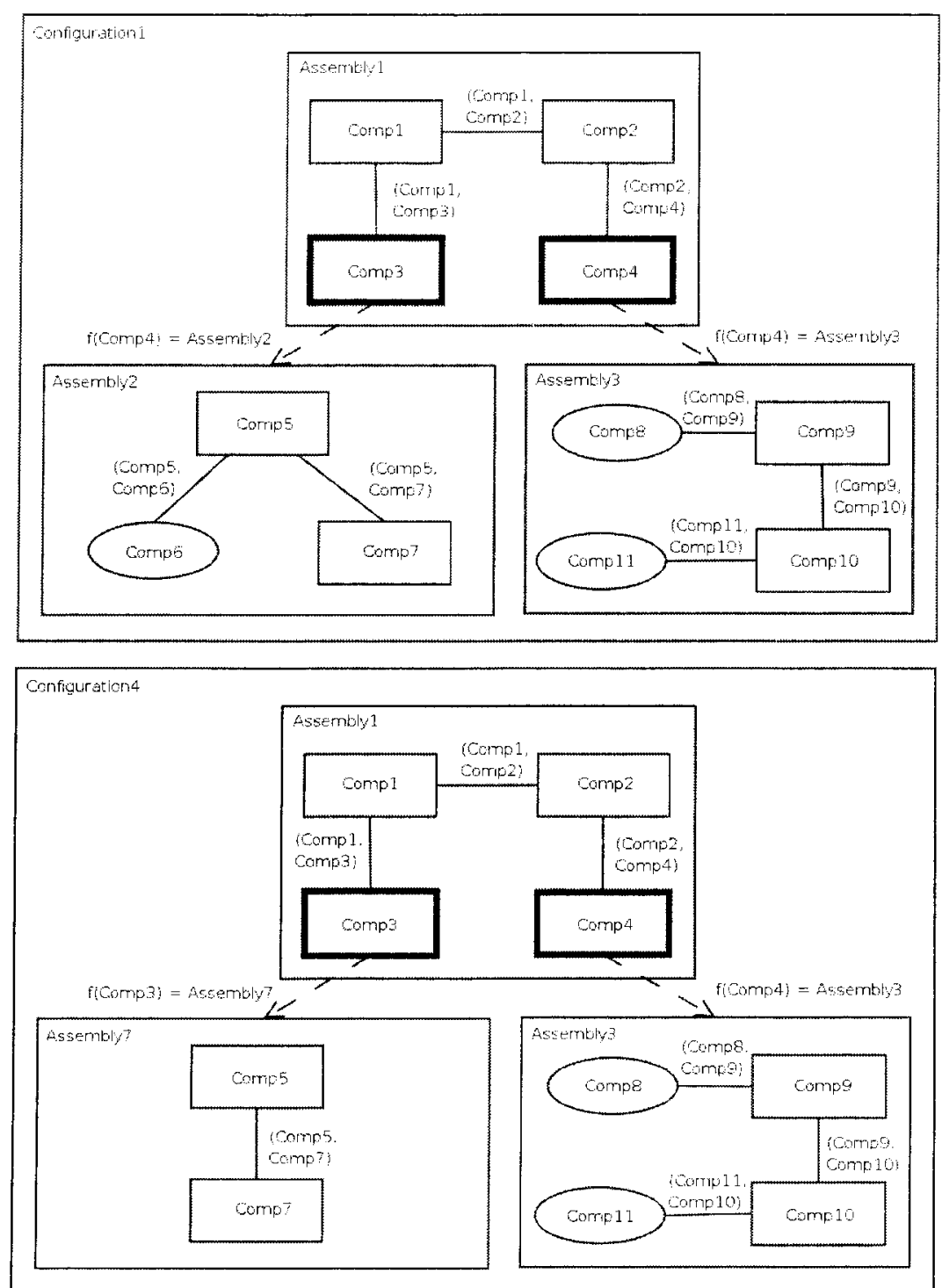

Figure 4.8: Specialization of a configuration by replacing a virtual component with an existing component. 


\section{Virtual Component Replaced with Existing Concrete or Composite Com- ponent}

To demonstrate replacing a virtual component with either an existing concrete or composite component, the configuration Configuration1 is specialized to produce the configuration Configuration 4 (Figure 4.8). This is accomplished by replacing the virtual component Comp6 in Assembly2 of Configuration1 with the concrete component Comp7 of Assembly7 in Configuration4. The rest of the components in Assembly? also exist in Assembly7.

The dependency (Comp5, Comp6) in Assembly2 of Configuration1 is replaced with the dependency (Comp5, Comp7) in Assembly7 of Configuration4. All other dependencies in Assembly2 also exist in Assembly\%.

All of the the constraints that are associated with the dependency (Comp5, Comp6) in Assembly2 become associated with the dependency (Comp5, Comp 7) in Assembly5. All constraints that are associated with the other dependencies in Assembly2 are associated with the same dependencies in Assembly?.

The rest of the assemblies in the original configuration, Configuration1, also exist in the new configuration, Configuration 4 .

\subsubsection{Definition}

A configuration, $A$, is a specialization of configuration $B$ if there is an assembly in $A$ and an assembly in $B$, such that the assembly in $A$ contains all of the components, dependencies and constraints of the assembly in $B$, with one exception: a virtual component in the assembly of $B$ has been replaced with a concrete or composite component in the assembly of $A$. Assembly $B$ may also contain additional constraints that have been discovered as a result of replacing a virtual component with a concrete or composite component. All other assemblies in configuration $A$ and configuration $B$ are the same. 
Definition 11 (Specialization). Given two valid configurations, $t_{1}, t_{2} \in \mathbb{T}$, where $t_{1}=\left(\boldsymbol{A}_{\mathbf{1}}, f_{1}\right)$ and $t_{2}=\left(\boldsymbol{A}_{\mathbf{2}}, f_{2}\right), t_{2}$ is a specialization of $t_{1}$ if and only if there exists an asscmbly $a_{i} \in \boldsymbol{A}_{\mathbf{1}}$ and an assembly $a_{i}^{\prime} \in \boldsymbol{A}_{\mathbf{2}}$. where $a_{i} \neq a_{i}^{\prime}$ and

$$
\begin{array}{r}
a_{i}=\left(\boldsymbol{C}_{\boldsymbol{i}}, \boldsymbol{D}_{\boldsymbol{i}}, \boldsymbol{Q}_{\boldsymbol{i}}, m_{i}\right) \\
\boldsymbol{C}_{\boldsymbol{i}}=\boldsymbol{P}_{\boldsymbol{i}} \cup \boldsymbol{V}_{\boldsymbol{i}} \cup \boldsymbol{Z}_{\boldsymbol{i}} \\
a_{i}^{\prime}=\left(\boldsymbol{C}_{\boldsymbol{i}}{ }^{\prime}, \boldsymbol{D}_{\boldsymbol{i}}{ }^{\prime}, \boldsymbol{Q}_{\boldsymbol{i}}{ }^{\prime}, m_{i}^{\prime}\right)
\end{array}
$$

such that:

1. All of the assemblies in $t_{1}$, except for $a_{i}$, must exist in $t_{2}$

2. If there is a composite component in $t_{1}$ that is mapped to the assembly $a_{i}$, then that composite component must map to the assembly $a_{i}^{\prime}$ in $t_{2}$. All other composite components in $t_{1}$ must map to the same assembly in $t_{2}$

3. A virtual component in $a_{i}$ is replaced with a concrete component in $a_{i}^{\prime}$, where the concrete component does not exist in $t_{1}$; or

4. A virtual component in $a_{i}$ is replaced with a composite component in $a_{i}^{\prime}$, where the composite component does not exist in $t_{1}$; or

5. A virtual component in $a_{i}$ is replaced with a concrete or composite component in $a_{i}^{\prime}$, where the concrete or composite component also exists in $a_{i}$

Each of these properties are explained in detail below.

1. If the assembly $a_{i}$ is removed from the set of assemblies in $t_{1}$, and the assembly $a_{i}^{\prime}$ is removed from the set of assemblies in $t_{2}$, then the remaining assemblies of $t_{1}$ must be a subset of the remaining assemblies of $t_{2}$. That is,

$$
\boldsymbol{A}_{\mathbf{1}}-\left\{a_{i}\right\} \subset \boldsymbol{A}_{\mathbf{2}}-\left\{a_{i}^{\prime}\right\}
$$


2. If there exists a composite component, $z \in \operatorname{COMPOSITE}\left(\boldsymbol{A}_{1}\right)$, that is mapped to the assembly $a_{i}$ by the relation $f_{1}$, then $z$ must be mapped to the assembly, $a_{i}^{\prime}$, by the relation $f_{2}$. That is,

$$
\text { If } \exists z \in \operatorname{COMPOSITE}\left(\boldsymbol{A}_{\mathbf{1}}\right) \text {, suchthat } f_{1}(z)=a_{i} \text { then } f_{2}(z)=a_{i}^{\prime}
$$

All other composite components that exist in both $t_{1}$ and $t_{2}$ must be mapped to the same assembly by the functions $f_{1}$ and $f_{2}$, respectively. That is,

$$
\forall z_{k} \in \operatorname{COMPOSITE}\left(\boldsymbol{A}_{\mathbf{1}}\right) \text { where } z_{k} \neq z, f_{1}\left(z_{k}\right)=f_{2}\left(z_{k}\right)
$$

3. There exists a virtual component, $v \in \boldsymbol{V}_{\boldsymbol{i}}$, in the assembly $a_{i}$, and a concrete component, $p \in \mathbb{P}-\operatorname{CONCRETE}\left(\boldsymbol{A}_{\mathbf{1}}\right)$, that is not contained in configuration $t_{1}$, such that $v$ is replaced by $p$ in the assembly $a_{i}^{\prime}$ (see Section 4.6.1), and $a_{i}^{\prime}$ has the following properties:

a) The set of components in $a_{i}^{\prime}$ is equal to set of components in $a_{i}$, minus the virtual component $v$, plus the concrete component $p$. That is,

$$
C_{i}^{\prime}=C_{i}-\{v\}+\{p\}
$$

b) Every dependency in the assembly $a_{i}$ that contains the virtual component $v$ must have a corresponding dependency in the assembly $a_{i}^{\prime}$ where $v$ is replaced by the concrete component $p$. That is, for every $c \in C_{i}$, where $c \neq v$,

$$
d=(c, v) \in \boldsymbol{D}_{i} \text { if and only if } d^{\prime}=(c, p) \in \boldsymbol{D}_{\boldsymbol{i}}^{\prime}
$$

All other dependencies in $a_{i}$ must also exist in $a_{i}^{\prime}$, such that for every $c_{1}, c_{2} \in \boldsymbol{C}_{\boldsymbol{i}}$, where $c_{1} \neq v$ and $c_{2} \neq v$,

$$
d=\left(c_{1}, c_{2}\right) \in \boldsymbol{D}_{i} \text { iff } d \in \boldsymbol{D}_{i}{ }^{\prime}
$$

c) All of the constraints in $a_{i}$ must also exist in $a_{i}^{\prime}$. That is,

$$
Q_{i} \subset Q_{i}{ }^{\prime}
$$


d) Each constraint in $a_{i}$ that is mapped to a dependency containing the virtual component, $v$, must be mapped to the corresponding dependency in $a_{i}^{\prime}$ that contains the concrete component, $p$. All other constraints in $a_{i}$ must be mapped to the same dependency in both $a_{i}$ and $a_{i}^{\prime}$. That is, for every $q \in Q_{i}$, if

$$
m_{i}(q)=d \quad \text { where } d=(v, c) \in \boldsymbol{D}_{\boldsymbol{i}}
$$

then

$$
m_{i}^{\prime}(q)=d^{\prime} \quad \text { where } d^{\prime}=(p, c)
$$

Otherwise, if

$$
m_{i}(q)=d \quad \text { where } d=\left(c_{1}, c_{2}\right) \in \boldsymbol{D}_{\boldsymbol{i}}, c_{1}, c_{2} \in \boldsymbol{C}_{\boldsymbol{i}},
$$

then

$$
m_{i}^{\prime}(q)=d
$$

4. There exists a virtual component, $v \in \boldsymbol{V}_{\boldsymbol{i}}$, in the assembly $a_{i}$, and a composite component, $z \in \mathbb{P}-\operatorname{COMPOSITE}\left(\boldsymbol{A}_{\mathbf{1}}\right)$, that is not contained in configuration $t_{1}$, such that $v$ is replaced by $z$ in the assembly $a_{i}^{\prime}$ (see Section 4.6.1), and $a_{i}^{\prime}$ has the following properties:

a) The set of components in $a_{i}^{\prime}$ is equal to set of components in $a_{i}$, minus the virtual component $v$, plus the composite component $z$. That is,

$$
C_{i}^{\prime}=C_{i}-\{v\}+\{z\}
$$

b) Every dependency in the assembly $a_{i}$ that contains the virtual component $v$ must have a corresponding dependency in the assembly $a_{i}^{\prime}$ where $v$ is replaced by the composite component $z$. That is, for every $c \in \boldsymbol{C}_{\boldsymbol{i}}$, where $c \neq v$,

$$
d=(c, v) \in \boldsymbol{D}_{i} \text { if and only if } d^{\prime}=(c, z) \in \boldsymbol{D}_{\boldsymbol{i}}^{\prime}
$$

All other dependencies in $a_{i}$ must also exist in $a_{i}^{\prime}$, such that for every $c_{1}, c_{2} \in \boldsymbol{C}_{\boldsymbol{i}}$, where $c_{1} \neq v$ and $c_{2} \neq v$,

$$
d=\left(c_{1}, c_{2}\right) \in D_{i} \text { iff } d \in D_{i}{ }^{\prime}
$$


c) All of the constraints in $a_{i}$ must also exist in $a_{i}^{\prime}$. That is,

$$
Q_{i} \subset Q_{i}^{\prime}
$$

d) Each constraint in $a_{i}$ that is mapped to a dependency containing the virtual component, $v$, must be mapped to the corresponding dependency in $a_{i}^{\prime}$ that contains the composite component, $z$. All other constraints in $a_{i}$ must be mapped to the same dependency in both $a_{i}$ and $a_{i}^{\prime}$. That is, for every $q \in Q_{i}$, if

$$
m_{i}(q)=d \quad \text { where } d=(v, c) \in \boldsymbol{D}_{\boldsymbol{i}}
$$

then

$$
m_{i}^{\prime}(q)=d^{\prime} \quad \text { where } d^{\prime}=(z, c) \in \boldsymbol{D}_{i}^{\prime}
$$

Otherwise, if

$$
m_{i}(q)=d \quad \text { where } d=\left(c_{1}, c_{2}\right) \in \boldsymbol{D}_{\boldsymbol{i}}, c_{1}, c_{2} \in \boldsymbol{C}_{\boldsymbol{i}},
$$

then

$$
m_{i}^{\prime}(q)=d
$$

5. There exists a virtual component, $v \in \boldsymbol{V}_{\boldsymbol{i}}$, in the assembly $a_{i}$, and a concrete or composite component, $y \in \boldsymbol{P}_{\boldsymbol{i}} \cup \boldsymbol{Z}_{\boldsymbol{i}}$, that is contained in the configuration $t_{1}$, such that $v$ is replaced by $y$ in the assembly $a_{i}^{\prime}$ (see Section 4.6.1), and $a_{i}^{\prime}$ has the following properties:

a) The set of components in $a_{i}^{\prime}$ is equal to set of components in $a_{i}$, minus the virtual component $v$. That is,

$$
C_{i}^{\prime}=C_{i}-\{v\}
$$

b) Every dependency in the assembly $a_{i}$ that contains the virtual component $v$ must have a corresponding dependency in the assembly $a_{i}^{\prime}$ where $v$ is replaced by the component $y$. That is, for every $c \in \boldsymbol{C}_{\boldsymbol{i}}$, where $c \neq v$,

$$
d=(c, v) \in D_{i} \text { if and only if } d^{\prime}=(c, y) \in D_{i}^{\prime}
$$


All other dependencies in $a_{i}$ must also exist in $a_{i}^{\prime}$, such that for every $c_{1}, c_{2} \in C_{i}$, where $c_{1} \neq v$ and $c_{2} \neq v$,

$$
d=\left(c_{1}, c_{2}\right) \in D_{i} \text { iff } d \in D_{i}{ }^{\prime}
$$

c) All of the constraints in $a_{i}$ must also exist in $a_{i}^{\prime}$. Therefore,

$$
Q_{i} \subset Q_{i}{ }^{\prime}
$$

d) Each constraint in $a_{i}$ that is mapped to a dependency containing the virtual component, $v$, must be mapped to the corresponding dependency in $a_{i}^{\prime}$ that contains the component, $y$. All other constraints in $a_{i}$ must be mapped to the same dependency in both $a_{i}$ and $a_{i}^{\prime}$. That is, for every $q \in Q_{i}$, if

$$
m_{i}(q)=d \quad \text { where } d=(v, c) \in \boldsymbol{D}_{\boldsymbol{i}}
$$

then

$$
m_{i}^{\prime}(q)=d^{\prime} \quad \text { where } d^{\prime}=(y, c) \in D_{i}^{\prime}
$$

Otherwise, if

$$
m_{i}(q)=d \quad \text { where } d=\left(c_{1}, c_{2}\right) \in \boldsymbol{D}_{\boldsymbol{i}}, c_{1}, c_{2} \in \boldsymbol{C}_{\boldsymbol{i}},
$$

then

$$
m_{i}^{\prime}(q)=d
$$

Using the definition above, it is possible to deduce several properties of specializations.

Given a sequence of configurations, (Conf1, Conf2, ..., ConfN), where a configuration in the sequence is a specialization of the configuration that precedes it in the sequence (e.g. Conf2 is a specialization of Conf1), then the configuration Conf1 cannot be a specialization of ConfN. In order to demonstrate this fact, it is necessary to prove several other properties of specializations.

As discussed in Section 4.6.1, given two configurations, Conf1 and Conf2, there are three possible cases for Conf 2 to be a specialization Conf1: 
1. A virtual component in Conf1 is replaced with a new concrete component in Conf?

2. A virtual component in Conf1 is replaced with a new composite component in Conf?

3. A virtual component in Conf1 is replaced with an existing concrete or composite component in Conf?

In the first and third cases, the number of assemblies in Confo is equal to the number of assemblies in Conf1 as the replacement of a virtual component with a concrete component does not introduce a new assembly. Also, the replacement of a virtual component with an existing composite component does not introduce a new assembly since the decomposition assembly of the composite component has already been defined in Conf1. In the second case, the number of assemblies in Conf2 is greater than the number of assemblies in Conf1 as the replacement of the virtual component with a new composite component results in at least one new assembly being added to the configuration. This new assembly is the decomposition assembly of the newly added composite component.

Therefore, given two configurations, Conf1 and Conf2, such that Conf2 is a specialization of Conf1, then the number of assemblies in configuration Conf1 must be less than or equal to the number of assemblies in configuration Conf2. This proof is presented formally below.

Lemma 3. Given two configurations, $t_{i}=\left(\boldsymbol{A}_{\boldsymbol{i}}, f_{i}\right), t_{j}=\left(\boldsymbol{A}_{\boldsymbol{j}}, f_{j}\right) \in \mathbb{T}$, where $t_{j}$ is a specialization of $t_{i}$, then the number of assemblies in $t_{i}$ must be less than or equal to the number of assemblies in $t_{j}$. That is,

$$
\left|\boldsymbol{A}_{i}\right| \leqslant\left|\boldsymbol{A}_{j}\right|
$$

Proof: Given two configurations, $t_{i}=\left(\boldsymbol{A}_{\boldsymbol{i}}, f_{i}\right), t_{j}=\left(\boldsymbol{A}_{\boldsymbol{j}}, f_{j}\right) \in \mathbb{T}$, where $t_{j}$ is a specialization of $t_{i}$, then by the definition of a specialization (see Definition 11) 
there exists an assembly, $a_{i} \in \boldsymbol{A}_{\boldsymbol{i}}$, and an assembly, $a_{j} \in \boldsymbol{A}_{\boldsymbol{j}}$, such that

$$
\boldsymbol{A}_{i}-a_{i} \subset \boldsymbol{A}_{j}-a_{j}
$$

This implies

$$
\left|\boldsymbol{A}_{\boldsymbol{i}}\right| \leqslant\left|\boldsymbol{A}_{j}\right|
$$

$Q E D$

In the case where the number of assemblies is equal in both Conf1 and Conf?, then it can de deduced that the number of virtual components in Conf2 is less than the number of virtual components in Conf1. This is because there is an assembly, Assembly1, in Conf1, and a corresponding assembly, Assembly2, in Conf2 such that a virtual component in Assembly1 has been replaced by either a new concrete component or an existing concrete or composite component in Assembly2, with all other assemblies being the same in both configurations.

Therefore, if Conf2 is a specialization of Conf1, and the number of assemblies in Conf1 is equal to the number of assemblies in Conf2, then the total number of virtual components in Conf2 is less than the total number of virtual components in Conf1.

Lemma 4. Given two configurations, $t_{i}=\left(\boldsymbol{A}_{\boldsymbol{i}}, f_{i}\right), t_{j}=\left(\boldsymbol{A}_{\boldsymbol{j}}, f_{j}\right) \in \mathbb{T}$, such that $t_{j}$ is a specialization of $t_{i}$, if the number of assemblies in $t_{i}$ is equal to the number of assemblies in $t_{j}$, then the total number of virtual components in $t_{j}$ is less than the total number virtual components in $t_{i}$. That is, if

$$
\left|\boldsymbol{A}_{i}\right|=\left|\boldsymbol{A}_{j}\right|
$$

then

$$
\left|V I R T U A L\left(\boldsymbol{A}_{j}\right)\right|<\left|V I R T U A L\left(\boldsymbol{A}_{i}\right)\right|
$$

Proof: Given two configurations, $t_{i}=\left(\boldsymbol{A}_{\boldsymbol{i}}, f_{i}\right), t_{j}=\left(\boldsymbol{A}_{j}, f_{j}\right) \in \mathbb{T}$, such that $t_{j}$ is a specialization of $t_{i}$, then there exists an assembly, $a_{i} \in \boldsymbol{A}_{\boldsymbol{i}}$, and an assembly, 
$a_{j} \in \boldsymbol{A}_{j}$ : where

$$
\begin{array}{r}
a_{i}=\left(\boldsymbol{C}_{\boldsymbol{i}}, \boldsymbol{D}_{\boldsymbol{i}}, \boldsymbol{Q}_{\boldsymbol{i}}, m_{i}\right) \\
\boldsymbol{C}_{\boldsymbol{i}}=\boldsymbol{P}_{\boldsymbol{i}} \cup \boldsymbol{V}_{\boldsymbol{i}} \cup \boldsymbol{Z}_{i} \\
a_{j}=\left(\boldsymbol{C}_{\boldsymbol{j}}, \boldsymbol{D}_{\boldsymbol{j}}, \boldsymbol{Q}_{\boldsymbol{j}}, m_{j}\right) \\
\boldsymbol{C}_{\boldsymbol{j}}=\boldsymbol{P}_{\boldsymbol{j}} \cup \boldsymbol{V}_{\boldsymbol{j}} \cup \boldsymbol{Z}_{\boldsymbol{j}}
\end{array}
$$

such that the properties of specialization hold.

The number of virtual components in the assembly $a_{j}$ must be less than the number of virtual components in $a_{i}$, by the definition of specializations (see Definition 11). That is,

$$
\left|\boldsymbol{V}_{j}\right|<\left|\boldsymbol{V}_{\boldsymbol{i}}\right|
$$

If the cardinality of the set of assemblies in $t_{i}$ is equal to the cardinality of the set of assemblies in $t_{j}$, then the following is true by Definition 11:

$$
\boldsymbol{A}_{\boldsymbol{i}}-a_{i}=\boldsymbol{A}_{j}-a_{j}
$$

Therefore,

$$
\left|V I R T U A L\left(\boldsymbol{A}_{\boldsymbol{i}}-a_{i}\right)\right|=\left|V I R T U A L\left(\boldsymbol{A}_{j}-a_{j}\right)\right|
$$

and it follows that

$$
\begin{gathered}
\left|V I R T U A L\left(\boldsymbol{A}_{j}-a_{j}\right)+\boldsymbol{V}_{j}\right|<\left|V I R T U A L\left(\boldsymbol{A}_{\boldsymbol{i}}-a_{i}\right)+\boldsymbol{V}_{\boldsymbol{i}}\right| \\
\left|\operatorname{VIRTUAL}\left(\boldsymbol{A}_{\boldsymbol{j}}\right)\right|<\left|V I R T U A L\left(\boldsymbol{A}_{\boldsymbol{i}}\right)\right|
\end{gathered}
$$

$Q E D$

The above properties are specific to the case where a single configuration is a specialization of another configuration. It is possible to generalize these properties for a sequence of configurations, (Conf1, Conf2, ..., ConfN), where a configuration in the sequence is a specialization of the configuration that precedes it in the sequence (ie. Conf 2 is a specialization of Conf1).

Lemma 3 may be generalized such that the number of assemblies in Conf1 must be less than or equal to the number of assemblies in ConfN. 
Lemma 5. Given a sequence of configurations, $\left(t_{1}, t_{2}, \ldots, t_{n}\right), n>1, t_{j}=\left(\boldsymbol{A}_{\boldsymbol{j}}, f_{j}\right)$, such that the configuration $t_{i+1}$ is a specialization of the configuration $t_{i}, 0<i<n$. then the number of assemblies in $t_{1}$ is less than or equal to the number of assemblies in $t_{n}$. That is,

$$
\left|\boldsymbol{A}_{1}\right| \leqslant\left|\boldsymbol{A}_{n}\right|
$$

Proof: Given a sequence of configurations, $\left(t_{1}, t_{2}, \ldots, t_{n}\right), n>1, t_{j}=\left(\boldsymbol{A}_{\boldsymbol{j}}, f_{j}\right)$, such that the configuration $t_{i+1}$ is a specialization of the configuration $t_{i}, 0<i<n$, then, by Lemma 3 ,

$$
\left|\boldsymbol{A}_{\mathbf{1}}\right| \leqslant\left|\boldsymbol{A}_{\mathbf{2}}\right| \leqslant \ldots \leqslant\left|\boldsymbol{A}_{\boldsymbol{n}}\right|
$$

This implies

$$
\left|\boldsymbol{A}_{1}\right| \leqslant\left|\boldsymbol{A}_{n}\right|
$$

QED

Lemma 4 may be generalized such that in the case where Conf1 and $N$ have the same number of assemblies, the total number of virtual components in ConfN must be less than the total number of virtual components in Conf1.

Lemma 6. Given a sequence of configurations, $\left(t_{1}, t_{2}, \ldots, t_{n}\right), n>1, t_{i}=\left(\boldsymbol{A}_{i}, f_{i}\right)$, such that the configuration $t_{i+1}$ is a specialization of the configuration $t_{i}, 0<i<n$, if the size of the set of assemblies in $t_{n}$ is equal to the size of the set of assemblies in $t_{1}$, then the total number of virtual components in $t_{n}$ must be less than the total number of virtual components in $t_{1}$. That is, if

$$
\left|\boldsymbol{A}_{n}\right|=\left|\boldsymbol{A}_{\mathbf{1}}\right|
$$

then

$$
\left|\operatorname{IRTU} A L\left(\boldsymbol{A}_{\boldsymbol{n}}\right)\right|<\left|\operatorname{VIRTU} A L\left(\boldsymbol{A}_{1}\right)\right|
$$


Proof: Given a set of configurations, $\left.t_{1}, t_{2}, \ldots, t_{n}\right), n>1, t_{i}=\left(\boldsymbol{A}_{i}, f_{i}\right)$, such that the configuration $t_{i+1}$ is a specialization of the configuration $t_{i}, 0<i<n$, if the number of assemblies in $t_{n}$ is equal to the number of assemblies in $t_{1}$, then, by Lemma 3 ,

$$
\left|\boldsymbol{A}_{1}\right|=\left|\boldsymbol{A}_{\mathbf{2}}\right|=\ldots=\left|\boldsymbol{A}_{\boldsymbol{n}}\right|
$$

Therefore, by Lemma 4,

$$
\left|V I R T U A L\left(\boldsymbol{A}_{\boldsymbol{n}}\right)\right|<\left|\operatorname{VIRTU} A L\left(\boldsymbol{A}_{\boldsymbol{n}-\mathbf{1}}\right)\right|<\ldots<\left|\operatorname{IRTU} A L\left(\boldsymbol{A}_{\mathbf{1}}\right)\right|
$$

This implies that

$$
|| \operatorname{IRTU} A L\left(\boldsymbol{A}_{\boldsymbol{n}}\right)|<| V I R T U A L\left(\boldsymbol{A}_{\mathbf{1}}\right) \mid
$$

$Q E D$

Using the properties above, it is possible to conclude that given a sequence of configurations, (Conf1, Conf2, ..., ConfN), where a configuration in the sequence is a specialization of the configuration that precedes it in the sequence (ie. Confo is a specialization of Conf1), then the configuration ConfN cannot be a specialization of Conf1. This property is relevant to show that once a virtual component has been replaced with either a concrete or composite component, it is not possible to create a specialization that contains the previously replaced virtual component.

This is proven in the following thereom using the above lemmas.

Theorem 2. Given a sequence of configurations, $\left(t_{1}, t_{2}, \ldots, t_{n}\right), n>1, t_{i}=\left(\boldsymbol{A}_{\boldsymbol{i}}, f_{i}\right)$, such that the configuration $t_{i+1}$ is a specialization of the configuration $t_{i}, 0<i<n$, then the configuration $t_{n}$ is not a specialization of $t_{1}$.

Proof: Given a sequence of configurations, $\left(t_{1}, t_{2}, \ldots, t_{n}\right), n>1, t_{i}=\left(\boldsymbol{A}_{\boldsymbol{i}}, f_{i}\right)$, such that the configuration $t_{i+1}$ is a specialization of the configuration $t_{i}, 0<i<n$, then by Lemma 5 ,

$$
\left|\boldsymbol{A}_{n}\right| \geqslant\left|\boldsymbol{A}_{1}\right|
$$

If the number of assemblies in $t_{n}$ is greater than the number of assemblies in $t_{1}$, then by Lemma $3, t_{1}$ is not a specialization of $t_{n}$ 
If the number of assemblies in $t_{n}$ is equal to the number of assemblies in $t_{1}$, then by Lcmma 6

$$
\left|\operatorname{IRTUAL}\left(\boldsymbol{A}_{n}\right)\right|<\left|V I R T U A L\left(\boldsymbol{A}_{\mathbf{1}}\right)\right|
$$

Thus, by Lemma $4, t_{1}$ is not a specialization of $t_{n}$.

$Q E D$

Another property of specializations is that each constraint in a configuration must also exist in a specialization of the configuration. This property is important as it demonstrates that all constraints that have been specified in a configuration are maintained throughout the specialization process. This prevents constraints from having to be redefined for each configuration.

This property is proven in the following theorem.

Theorem 3. Given:

1. Two configurations, $t, t^{\prime} \in \mathbb{T}$, where $t=(\boldsymbol{A}, f)$ and $t^{\prime}=\left(\boldsymbol{A}^{\prime}, f^{\prime}\right)$, such that $t^{\prime}$ is a specialization of $t$

2. CONSTRAINT $(\boldsymbol{A})$ is the set of all constraints in $t$

3. CONSTRAINT $\left(\boldsymbol{A}^{\prime}\right)$ is the set of all constraints in $t^{\prime}$

Then,

$$
\operatorname{CONSTRAINT}(\boldsymbol{A}) \subset \operatorname{CONSTRAINT}\left(\boldsymbol{A}^{\prime}\right)
$$

Proof: If the configuration $t^{\prime}=\left(\boldsymbol{A}^{\prime}, f^{\prime}\right)$ is a specialization of the configuration $t=(\boldsymbol{A}, f)$, then there exists an assembly, $a \in \boldsymbol{A}$, and an assembly, $a^{\prime} \in \boldsymbol{A}^{\prime}$, where

$$
\begin{array}{r}
a=(\boldsymbol{C}, \boldsymbol{D}, \boldsymbol{Q}, m) \\
a^{\prime}=\left(\boldsymbol{C}^{\prime}, \boldsymbol{D}^{\prime}, \boldsymbol{Q}^{\prime}, m^{\prime}\right)
\end{array}
$$

such that

$$
\boldsymbol{A}-\{a\} \subset \boldsymbol{A}^{\prime}-\left\{a^{\prime}\right\}
$$


This implies that the set of constraints CONSTRAINT $(\boldsymbol{A}-\{a\})$ is a subset of the set of constraints CONSTRAINT $\left(\boldsymbol{A}^{\prime}-\left\{a^{\prime}\right\}\right)$. That is,

$$
\text { CONSTRAINT }(\boldsymbol{A}-\{a\}) \subset \operatorname{CONSTRAINT}\left(\boldsymbol{A}^{\prime}-\left\{a^{\prime}\right\}\right)
$$

By the definition of a specialization (see Definition 11), the set of constraints, $Q$, in $a$ is a subset of the set of constraints, $Q^{\prime}$ in $a^{\prime}$. Thus,

$$
Q \subset Q^{\prime}
$$

It follows that

$$
\boldsymbol{Q} \cup \operatorname{CONSTRAINT}(\boldsymbol{A}-\{a\}) \subset \boldsymbol{Q}^{\prime} \cup \operatorname{CONSTRAINT}\left(\boldsymbol{A}^{\prime}-\left\{a^{\prime}\right\}\right)
$$

The set of all constraints in $t, \operatorname{CONSTRAINT}(\boldsymbol{A})$, is equal to the set of constraints in $a, \boldsymbol{Q}$, plus the set of constraints in $\boldsymbol{A}-a, \operatorname{CONSTRAINT}(\boldsymbol{A}-\{a\})$. That is,

$$
\operatorname{CONSTRAinT}(\boldsymbol{A})=\boldsymbol{Q} \cup \operatorname{CONSTRAinT}(\boldsymbol{A}-\{a\})
$$

The set of all constraints in $t^{\prime}, \operatorname{CONSTRAINT}\left(\boldsymbol{A}^{\prime}\right)$, is equal to the set of constraints in $a^{\prime}, \boldsymbol{Q}^{\prime}$, plus the set of constraints in $\boldsymbol{A}^{\prime}-a^{\prime}, \operatorname{CONSTRAINT}\left(\boldsymbol{A}^{\prime}-\left\{a^{\prime}\right\}\right)$. That is,

$$
\operatorname{CONSTRAINT}\left(\boldsymbol{A}^{\prime}\right)=\boldsymbol{Q}^{\prime} \cup \operatorname{CONSTRAINT}\left(\boldsymbol{A}^{\prime}-\left\{a^{\prime}\right\}\right)
$$

This implies

$$
\operatorname{CONSTRAINT}(\boldsymbol{A}) \subset \operatorname{CONSTRAINT}\left(\boldsymbol{A}^{\prime}\right)
$$

Therefore, if the configuration $t^{\prime}$ is a specialization of the configuration $t$, then the set of constraints in $t$ is a subset of the set of constraints in $t^{\prime}$.

$Q E D$

\subsubsection{Graphical Notation}

There is no graphical representation of a specialization. 


\subsection{Specialization Scheme}

\subsubsection{Description}

As multiple instances of a software system are produced. a deployable configuration must be created to represent each instance of the system. Creating each deployable configuration may require a large number of specializations to be defined to reflect the replacement of each virtual component with either a concrete or composite component. This results in a large number of configurations being created for the entire system. In order to maintain all of the configurations, a specialization scheme is used. A specialization scheme contains all of the configurations that have been defined for a software system.

Each specialization scheme must contain a base configuration. The base configuration is the configuration in the specialization scheme that contains the most virtual components. All other configurations in the specialization scheme represent specializations that reflect the replacement of a virtual component with either a concrete or composite component.

\subsubsection{Definition}

A specialization scheme consists of a set of valid configurations such that:

- There exists exactly one configuration that is not a specialization of any other configuration in the specialization scheme. This configuration is called the base configuration.

- Every configuration, besides the base configuration, is a specialization of at least one other configuration in the specialization scheme.

Definition 12 (Specialization Scheme). A specialization scheme, s, is a finite set of configurations, $\boldsymbol{T} \subset \mathbb{T}$, such that: 
1. There exists a base configuration, $t_{b} \in \boldsymbol{T}$, that is not a specialization of any other configuration in $\boldsymbol{T}$. Thus,

$\exists t_{b} \in \boldsymbol{T}$ such that $\forall t \in \boldsymbol{T}$, where $t_{b} \neq t, t_{b}$ is not a specialization of $t$

2. Every configuration, $t \in \boldsymbol{T}$, besides the base configuration, $t_{b}$, is a specialization of at least one other configuration, $t_{s} \in \boldsymbol{T}$. Thus,

$\forall t \in \boldsymbol{T}, t \neq t_{b}, \exists t_{s} \in \boldsymbol{T}$ such that $t$ is a specialization of $t_{s}$

A specialization scheme may be represented using a directed graph where the vertices of the graph represent the configurations of the specialization scheme, and the edges of the graph represent the specialization relationship between two configurations.

Definition 13 (Specialization Scheme Graph). The specialization scheme graph, $\boldsymbol{G}=(\boldsymbol{V}, \boldsymbol{E})$, of the specialization scheme, $s=\boldsymbol{T}$, is a directed graph where

1. The set of vertices, $\boldsymbol{V}$, of $g$ is equal to the set of configurations in $s$. That is,

$$
V=T
$$

2. $\boldsymbol{E}$ is the set of edges of $\boldsymbol{G}$, such that $\left(t_{1}, t_{2}\right)$ is an edge of $\boldsymbol{E}$, where $t_{1}, t_{2} \in \boldsymbol{T}$. if and only if $t_{2}$ is a specialization of $t_{1}$. Thus,

$$
\left(t_{1}, t_{2}\right) \in \boldsymbol{E}, t_{1}, t_{2} \in \boldsymbol{T} \text {, iff } t_{2} \text { is a specialization of } t_{1}
$$

Using the definition of a specialization scheme, and the properties of specialization described in Section 4.6, it is possible to conclude that a specialization scheme graph is a connected, acyclic graph. 
Theorem 4. Given a specialization scheme, $s=\boldsymbol{T}$, the specialization scheme graph, $\boldsymbol{G}=(\boldsymbol{V}, \boldsymbol{E})$, of $s$ is a connected, acyclic graph.

Proof: This will be proven by contradiction.

First, assume that $\boldsymbol{G}$ is cyclic. Thus, there exists a vertex, $v_{1} \in \boldsymbol{V}$, and a set of edges, $\boldsymbol{E}_{\boldsymbol{p}} \subset \boldsymbol{E}$, such that if you start at vertex $v_{1}$ and traverse the edges of $\boldsymbol{E}_{\boldsymbol{p}}$, then you will arrive back at vertex $v_{1}$. Thus,

$$
\boldsymbol{E}_{\boldsymbol{p}}=\left\{\left(v_{1}, v_{2}\right),\left(v_{2}, v_{3}\right), \ldots,\left(v_{n-1}, v_{n}\right),\left(v_{n}, v_{1}\right)\right\}, v_{i} \in \boldsymbol{V}
$$

Each vertex, $v_{i} \in \boldsymbol{V}$, represents a configuration, $t_{i} \in \boldsymbol{T}$, and each edge, $\left(v_{j}, v_{k}\right) \in \boldsymbol{E}$, represents a specialization relationship between the configurations $t_{j}, t_{k} \in \boldsymbol{T}$, such that $t_{k}$ is a specialization of $t_{j}$.

Thus, the set of edges, $\boldsymbol{E}_{\boldsymbol{p}}$, that represents a cycle in $\boldsymbol{G}$, implies that there exists a set of configurations, $\left\{t_{1}, t_{2}, t_{3}, \ldots, t_{n-1}, t_{n}\right\}, t_{i} \in \boldsymbol{T}$, such that

$$
\begin{gathered}
t_{2} \text { is a specialization of } t_{1} \\
t_{3} \text { is a specialization of } t_{2} \\
t_{n} \text { is a specialization of } t_{n-1} \\
t_{1} \text { is a specialization of } t_{n}
\end{gathered}
$$

By Theorem 2, $t_{1}$ cannot be a specialization of $t_{n}$, thus, a the existence of a cycle contradicts the fact that $s$ is a specialization scheme.

Next, assume that $\boldsymbol{G}$ is acyclic, but not connected. This implies that there are at least two vertices, $v_{1}, v_{2} \in \boldsymbol{V}$, that have zero incoming edges. Thus,

$$
\exists v_{1}, v_{2} \in \boldsymbol{V} \text { such that degree } e_{i n}\left(v_{1}\right)=\operatorname{degree}_{\text {in }}\left(v_{2}\right)=0
$$

This implies that there are at least two configurations, $t_{1}, t_{2} \in \boldsymbol{T}$, that are not a specialization of another configuration. Thus,

$$
\exists t_{1}, t_{2} \in \boldsymbol{T}
$$


such that

$\forall t \in \boldsymbol{T}, t \neq t_{1}$ and $t \neq t_{2}$. neither $t_{1}$ or $t_{2}$ is a specialization of $t$

Thus, there are at least two base configurations for the specialization scheme, s. By definition, a specialization scheme has only one base configuration, therefore, this contradicts the fact that $s$ is a specialization scheme.

Thus, if $s$ is a specialization scheme, then the specialization scheme graph of $s$ is an acyclic, connected graph.

$Q E D$

\subsubsection{Graphical Notation}

The graphical representation of a specialization scheme is illustrated in Figure 4.9. An example of a specialization scheme is shown to illustrate the sequence of specializations created to obtain two separate deployable configurations from a single configuration, Configuration1, containing two virtual components (Figure 4.9). Each configuration is expanded to show all assembly, component and dependency information contained in the configuration. An arrow is used to depict the specialization relationship, with the arrow originating from the specialization configuration and extending to the configuration that has been specialized.

In the first specialization, Configuration?, the virtual component Virtual1 is replaced with the concrete component Concrete. The second specialization, Configuration 3 , is created by replacing the same virtual component, Virtual1, with a different concrete component, Concrete3. The third specialization, Configuration 4, is created by replacing the virtual component Virtual2 with the concrete component Concrete4.

The configuration Configuration. is then specialized by replacing the virtual component Virtual2 with the concrete component Concrete5. This results in the deployable configuration Configurations.

The configuration Configuration 3 is specialized by replacing the virtual component Virtuald with the concrete component Concrete4. This results in the deployable configuration Configurations. 


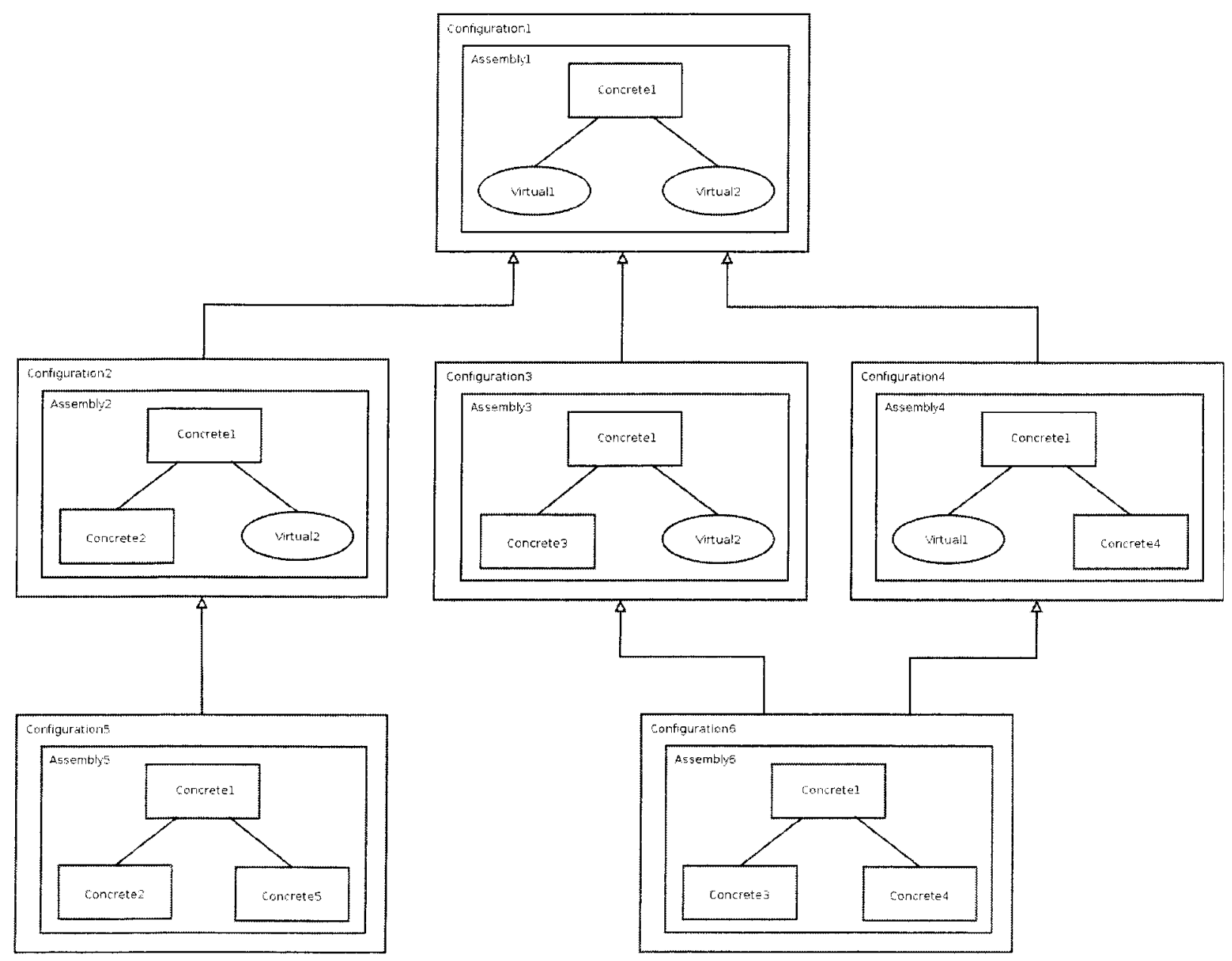

Figure 4.9: The graphical representation of a specialization scheme. The contents of each configuration are shown to illustrate how each specialization was created. The configuration, Configuration1, is the base configuration of this specialization scheme. 
The configuration Configuration 4 is specialized by replacing the virtual component Virtual1 with the concrete component Concrete3. This also results in the deployable configuration Configuration5.

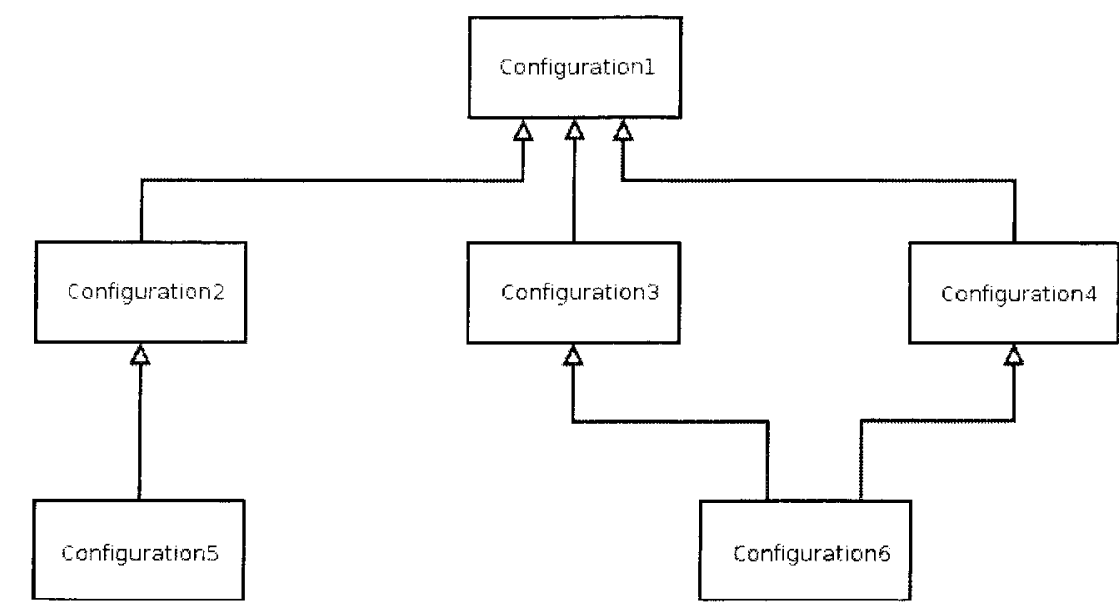

Figure 4.10: The specialization scheme graph of the specialization scheme shown in Figure 4.9 .

Figure 4.10 shows the specialization scheme graph of the specialization scheme illustrated in Figure 4.9. Each vertex of the graph represents a configuration, and each edge of the graph represents the specialization relationship between two configurations. An edge is depicted as an arrow with an empty head, with the arrow originating from the specialization configuration and extending to the configuration that has been specialized.

\subsection{Discussion}

Some of the elements and concepts presented within the formal specification could have been defined differently. These include:

- Defining dependencies as unordered pairs

- Requiring that components exist within a single assembly of a configuration 
- Requiring that a specialization replace only a single virtual component

This section provides a brief discussion as to why these aspects of the formal specification were defined the way they were, as well as to present several alternative methods for defining the same element or concept.

\section{Defining dependencies as unordered pairs}

The formal specification defines a dependency as an unordered pair of components. This allows a dependency to represent a relation between components without defining the nature of the relationship. The nature of the dependency is explicitly defined by associating constraints with the dependency.

An alternative approach for defining dependencies would be to represent a dependency as an ordered pair of components. Ordering the pair of components would implicitly add meaning to the relationship, as it shows the direction of the dependency. This may be useful in providing additional context to a person looking at the dependency model of a system; however, it is still the constraints that define the nature of the dependency.

Another alternative approach for defining dependencies would be to represent a dependency as a component set. This would allow for a dependency to be defined amongst a set of components, meaning that each component within the set is dependent upon all of the other components within the set. This would also allow for a dependency to be defined on a single component, thus constraints may, in effect, be assosciated with a component.

\section{Requiring that components exist within a single assembly of a configura- tion}

The formal specification requires that a component exist in a single assembly within a configuration. This restriction exists only at the model level, as several components within the dependency model may be represented by a single product in the physical deployment of a product. This unique component requirement was used in order to ensure that the structure of a configuration may be represented as a tree. 
Unfortunately, the tree structure of a configuration does not fit well in defining the layers of a software system. In order to overcome this limitation, the requirement of defining unique components within the assemblies of a configuration may be removed from the formal specification, allowing for the same component to exist in multiple assemblies.

In the case of composite components being used within the multiple assemblies, all instances of the composite component would reference the same decomposition assembly. Thus, a configuration would be represented as a directed graph; however, it would not be a tree structure. The benefit of this is that it allows for the layers of a system to be defined.

\section{Requiring that a specialization replace only a single virtual component}

The formal specification defines a specialization as replacing a single virtual component in a configuration with either a concrete or composite component, while maintaining all other information in the configuration. By allowing only a single virtual component to be replaced within a specialization, it requires that a separate specialization be defined each time that a decision is made to use an individual product in a software system. This allows for the maximum number of deployable configurations to be defined for a system.

It may be the case that when deciding upon the concrete and/or composite components to replace the virtual components, a situation arises where a particular combination of concrete/composite components must be used to produce a deployable configuration; using these components with any other set of components would not produce a configuration that could physically deployed. In this case, it may be desireable to create a single specialization that replaces all of the appropriate virtual components with the selected set of concrete and composite components.

Although the formal specification does not currently provide this functionality, this may be implemented within a tool. The tool could permit users to define a specialization that replaces several virtual components within a configuration, then, internally, the tool could automatically generate the individual specializations required to produce the specialization defined by the user. Thus, no changes are required within the 
formal specification in order to provide the desired functionality.

\subsection{Summary}

Chapter 3 described how developing a software system using the application assembly process may result in a large number of dependencies and constraints. Managing these dependencies and constraints presents several issues, and addressing these issues requires a process, as well as a set of tools to support the process.

This chapter presented a formal specification of modeling the products, dependencies and constraints within an assembled software system. The formal specification consisted of a set of elements that are used to model the hierarchical decomposition of a system. The elements include:

- components

- dependencies

- constraints

- assemblies

- configurations

- specializations

- specialization schemes

The following chapter describes how this formal specification is used as the basis for creating a process to manage dependencies and constraints in a software system. As well, the tool that was developed, as part of this research, to support the management process is introduced. 


\section{Chapter 5}

\section{Managing Dependencies and Constraints}

The application assembly process defined in Chapter 3 described how a software product is assembled from existing products and deployed to produce a system. Implementing a software system using the application assembly process results in a large number of dependencies and constraints that must be satisified in order for the system to function properly. The management of these dependencies and constraints is an important use case that must be carried out by all actors involved in the application assembly process. This use case presented the need for a formal specification for modeling an assembled software system.

To meet this requirement, Chapter 4 defined a formal specification for modeling the software products in a software system, as well as the dependencies and constraints that exist in the system.

This chapter explains the process of using the formal specification throughout the application assembly process to assist in the management of dependencies and constraints within an assembled software system. The activites involved in the management process are:

1. Define the system structure by creating configurations to model the components and dependencies that exist within the system. This involves defining a base configuration for the system. A set of specializations are then defined to reflect 
the sclection of different products to be used within the system.

2. Specify the constraints that exist in each configuration. This involves declaring each constraint as it is discovered by providing a unique identifier and a description for the constraint. Each declared constraint is then defined by specifying the procedure for checking that the constraint is satisfied.

3. Verify the constraints that exist in each configuration. This involves checking that each constraint that has been defined in a configuration is satisfied in an installed system.

Throughout the life cycle of a software system, these activities are continuously ongoing, and the activities may be executed concurrently by all actors involved with the system. The activities may be ongoing within different organizations, and they may be executed by developers, assemblers, deployers and users. Furthermore, those executing the activities may be separated geographically. Thus, it is important that the dependency and constraint management process be able to deal with this concurrency and geographic distribution amongst the actors involved with the process.

An example is provided in order to give a better understanding of the activities involved in using the formal specification for managing dependencies and constraints. The example application used throughout this chapter is the open source xPetstore [49] Java 2, Enterprise Edition (J2EE) [13] application. This is a port of the J2EE Blueprints Pet Store application [15] that uses the open source XDoclet [48] tool to automatically generate J2EE specific code and deployment descriptors. The xPetstore software product is available for download, and it may be deploved in different environments by using different application servers and databases.

The example presented defines a base configuration for the xPetstore system, as well as a set of specializations to reflect the selection of different products used to deploy the system. Two deployable configurations of the xPetstore system are defined to represent the instances of the xPetstore system that were installed throughout the example. Also, several constraints that were discovered while installing each deployable configuration are specified. 
A tool for assisting with the above activities is also presented. The tool is based upon the formal specification defined in Chapter 4, and it places an emphasis on using open collaboration to ease the constraint management process.

This chapter is separated into the following sections:

Section 5.1: This section explains how to model the structure of a software system. This involves creating a base configuration for the system and defining a set of specializations to reflect the decisions made in selecting products to be included within the assembled product and/or deployed system.

Section 5.2: This section describes how constraints are specified and added to configurations within the specialization scheme. A constraint must be first declared by providing a unique name and a description outlining the nature of the constraint. The constraint must then be defined by providing a rule set that is used to check that the constraint is satisfied.

Section 5.3: This section describes how a system is verified once it has been installed. An installed system is verified by manually checking each constraint defined within the appropriate deployable configuration to ensure that it is properly satisfied.

Section 5.4: This section discusses how the system structure and constraint information may be shared amongst the organizations that produce products and the organizations that assemble these products into a larger product. Using the Internet as the communication medium, it is much easier to share this information across physical boundaries.

Section 5.5: This section presents the tool that was developed as a proof of concept to assist with the constraint management process. The functionality of the tool is described, as well as future improvements that may be implemented within the tool.

\subsection{Define the System Structure}

The first activity involves defining the structure of the system. The Assembler and Deployer define the system structure by creating a specialization scheme for the system. The steps involved in creating the specialization scheme are:

1. The Assembler defines a base configuration for the system. 
2. The Assembler and Deployer create a set of specializations for the system based upon the decisions made while selecting software products to be included within the assembled product or the deployed system.

Each of these steps is discussed in the following sections.

\subsubsection{Base Configuration}

A base configuration is created to define the high level architecture of the system. This is created by analyzing the requirements of the product being assembled, dividing the product into components and allocating the functionality to the components. The services required by the product must also be defined as components within the system.

Once the functionality of the product being assembled has been divided into a set of components, and the services required by the product have been defined as components, the dependencies between the components must be defined. A dependency is defined for each relationship that exists between two components within the system.

Finally, the set of components must be defined as either concrete, composite, or virtual components within the base configuration. This should be done as follows:

- If there is only a single product available that provides the required functionality, and the product cannot be decomposed into a set of sub-products, then a concrete component should be used.

- If the product may be decomposed into a set of sub-products, each of which provides a specific functionality within the system, then a composite component should be used. In this case an assembly must be defined to represent the decomposition of the component.

- If more than one product exists that provides the required functionality, then a virtual component should be used. This represents a selection point that will allow for the base configuration to be specialized in the future once a decision has been made as to which product will be selected. 
The goal of the base configuration, when deciding how to allocate the types of components, is to provide as many selection points as possible. This allows for flexibility when defining specializations of the base configuration.

\subsubsection{Example: xPetstore Base Configuration}

The base configuration of the xPetstore system defines the components that are assembled to create the xPetstore product. It also defines the components that provide the services required by the xPetstore product for it to be deployed.

The top level of the hierarchical decomposition for the xPetstore system, which is represented by the RootAssembly assembly, defines two components: XPetstore and DeployEnv (Figure 5.1. The xPetstore component represents the assembled xPetstore product, and the DeployEnv component represents the services required by the xPetstore product in order to function properly. Both of these components are defined as composite components as they are decomposed into a set of sub-components.

The XPetstoreAssembly represents the decomposition of the XPetstore composite component. By reverse engineering the xPetstore software product, it was found that it is assembled from three components (Figure 5.1):

- a web frontend that provides a view of the pet store and handles customer requests (XPetstoreWeb)

- the business logic that manages the pet store (XPetstoreLogic)

- a domain model that maintains and persists the data of the pet store (XPetstoreDomain)

There is only a single option available for providing each of these functionalities, therefore, the components are defined as concrete components within the base configuration.

The web frontend handles a request from the customer, and it forwards the request to the business logic for further processing. The business logic accesses and/or modifies the data stored in the domain model, and then returns the result to the web frontend. Finally, the web frontend displays the response to the customer. Since the 
web frontend interacts directly with the business logic, there exists a dependency, (XPetstoreWeb, XPetstoreLogic), between these two products. There is also a dependency, (XPetstoreLogic, XPetstoreDomain), between the business logic and the domain model since the business logic directly accesses/modifies the data stored in the domain model.

The DeployEnvAssembly represents the decomposition of the DeployEnv composite component. The xPetstore product requires both a J2EE application scrver and a database to function properly, thus the J2EEAppServer and Database components are defined within the base configuration (Figure 5.1). There exists several products for each of these services, therefore, both the JEEEAppServer and Database components are defined as virtual components.

Since the J2EE application server is responsible for communicating with the database, there is a dependency, (J2EEAppServer, Database), between the J2EE application server and the database.

The graphical representation of the base configuration for the xPetstore system is shown in Figure 5.1, and the formal definition of the base configuration is given in Table 5.1.

The format of the formal defintion for the base configuration shown in Table 5.1 is as follows:

1. The row under the heading $t=(\boldsymbol{A}, f)$ contains the definition of the configuration. This includes:

a) The set of assemblies, $\boldsymbol{A}$, contained in the configuration.

b) The mapping from each composite component in the configuration to the assembly containing the decomposition of the composite component, as defined by the function $f$

2. The rows under the heading $a=(\boldsymbol{C}, \boldsymbol{D}, \boldsymbol{Q}, m)$ contains the definition of each assembly contained within the configuration. This includes:

a) The set of components, $C$, contained in the assembly

b) The dependency set, $D$, on the set of components, $C$ 


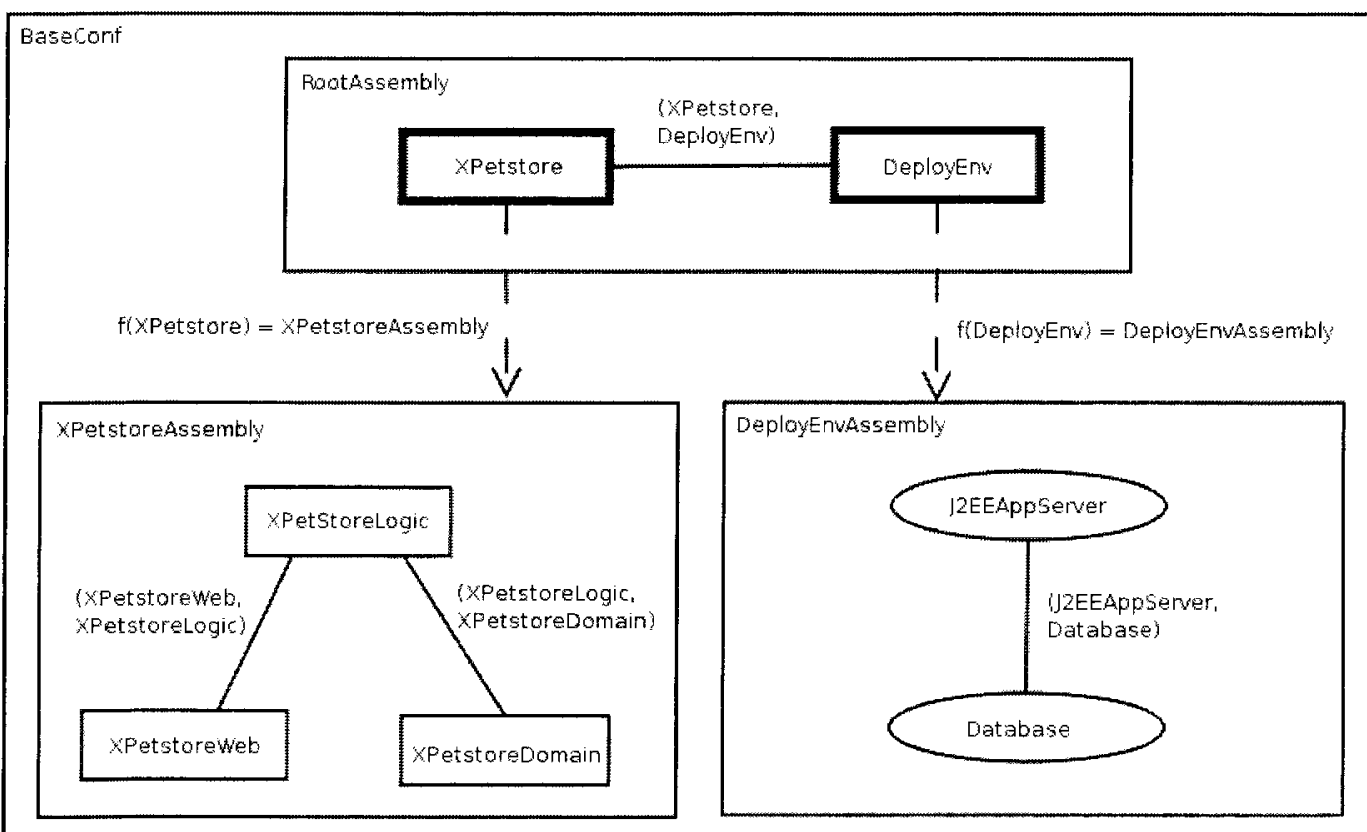

Figure 5.1: Base configuration for the xPetstore system. 


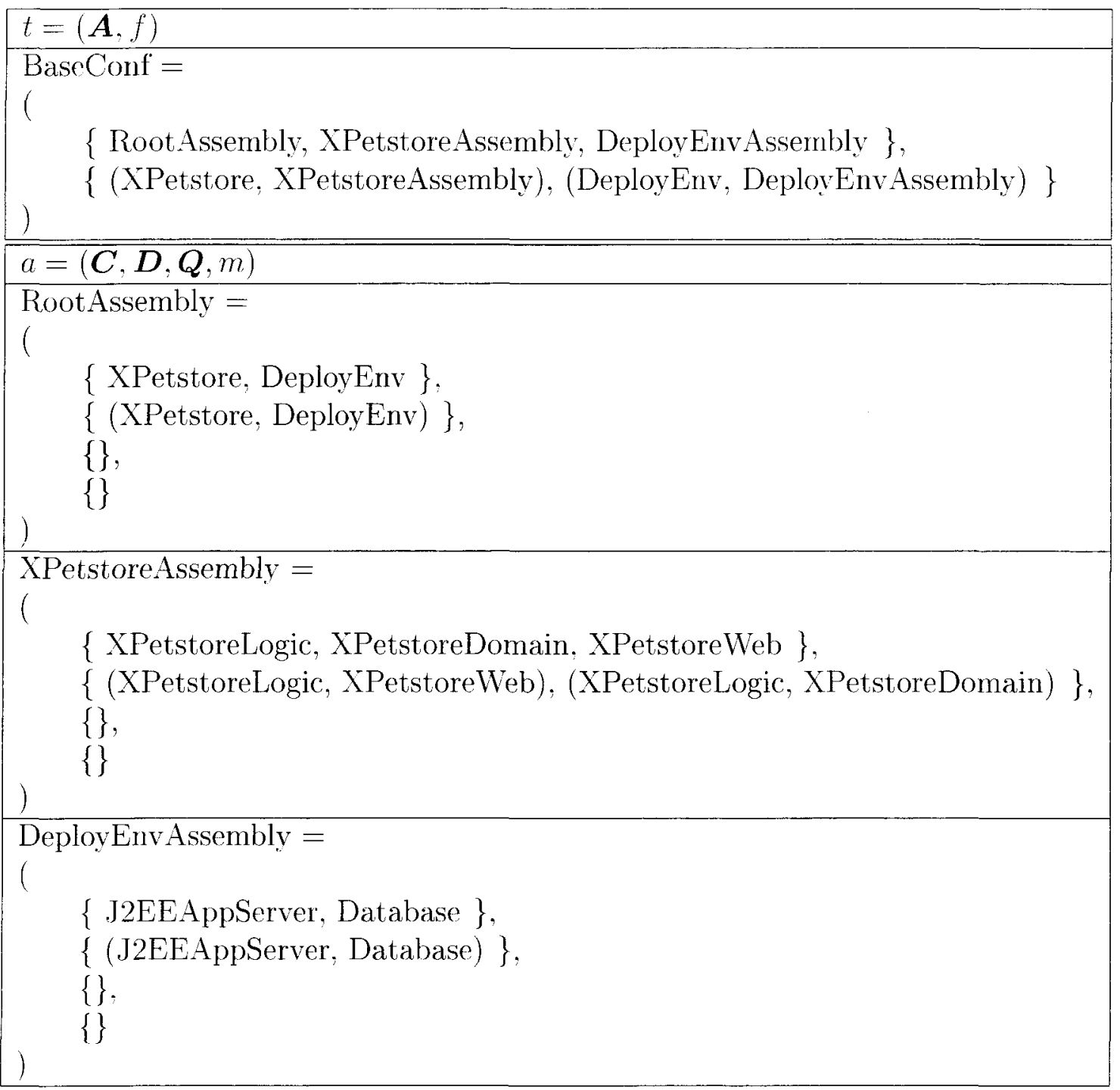

Table 5.1: The formal definition of the base configuration for the xPetstore system. 
c) The set of constraints, $\boldsymbol{Q}$, in contained in the assembly

d) The mapping from each constraint to the dependency in which the constraint is associated, as defined by the function $m$

\subsubsection{Creating Specializations}

The base configuration defines the structure of the system, but it does not represent a deployable system. A set of specializations must be defined by replacing each virtual component in the base configuration with either a concrete or composite component.

The Assembler and Deployer are responsible for creating specializations as they carry out the Select Software Product use case (see Section 3.1.2). Each time the Assembler or Deployer decides to use a particular software product within the system. a specialization must be created to reflect this decision. The specialization is added to the specialization scheme of the system.

A separate specialization should be created for each virtual component replacement, independent of all other component replacements. Thus, for every possible component that may be used within the system, the base configuration should be specialized to reflect the selection of each component. Then, a specialization should be created for each possible combination of components that may be used within the system. This is required in order for each configuration in the specialization scheme to contain constraints that are only applicable to the set of components contained within the configuration.

For example, consider a base configuration, BaseConf, that contains two virtual components, J2EEAppServer and Database. The specialization JBossConf is created by replacing the J2EEA ppServer virtual component in BaseConf with the JBoss concrete component. The specialization MySqlConf is created by replacing the Database virtual component in BaseConf with the MySql concrete component. The JBossConf and MySqlConf configurations would contain constraints that exist as a result of using the JBoss and MySql components, respectively.

If the JBoss and MySql components are to be used together within the system, then the specialization JBossMySqlConf is created by replacing the Database virtual 
component in JBossConf with the MySql concrete component, or by replacing the J2EEAppServer virtual component in MySqlConf with the JBoss concrete component. Any constraints that are added to the JBossMySqlConf configuration should only pertain to using the JBoss component in conjunction with the MySql component. Thus, each configuration should only contain constraints that are applicable to the components contained within the configuration. This is explained further in Section 5.2.

The process of creating specializations is repeated until all of the virtual components have been replaced with either concrete or composite components. The result is a deployable configuration. It is possible to create multiple deployable configurations for a system by creating different instances of the assembled software product and/or the deployed system. Different Assemblers may create multiple instances of the assembled software product by selecting different products to provide the same functionality within the assembled product. Different Deployers may create multiple instances of the deployed system by selecting different products to provide the same services required by the assembled product.

\subsubsection{Example: Creating Specializations for the xPetstore System}

In order to demonstrate the process of creating specializations to produce deployable configurations, two different instances of the xPetstore system were deployed.

The first instance of the xPetstore system uses JBoss [18] as the J2EE application server and HSQLDB [10] as the database. In order to produce the deployable configuration to represent this instance of the xPetstore system, several specializations need to be defined (Figure 5.2). The configuration JBossConf is created by replacing the J2EEAppServer virtual component, contained in the configuration BaseConf, with the JBoss concrete component. JBossConf is a specialization of BaseConf because the following properties hold:

1. The virtual component J2EEAppServer, in the assembly DeployEnvAssembly of BaseConf, is replaced with the concrete component JBoss, in the assembly 
JBossDeployEnvAssembly of JBossConf. such that:

a) The set of components in JBossDeployEnvAssembly contains all of the components in DeployEnvAssembly, except for the J2EEAppServer virtual component, which has been replaced by the JBoss concrete component.

b) The dependency (J2EEAppServer, Database) in DeployEnvAssembly is replaced with the dependency (JBoss, Database) in JBossDeployEnvAssembly to reflect the replaccment of the J2EEAppServer virtual component with the JBoss concrete component

2. All of the assemblies in BaseConf, except for DeployEnvAssembly, also exist in JBossConf

3. The composite component DeployEnv, which was mapped to the assembly DeployEnvAssembly in BaseConf, is mapped to the assembly JBossDeployEn$v A s s e m b l y$ in JBossConf; all other composite components are mapped to the same assemblies in both BaseConf and JBossConf

The properties of specializations that pertain to constraints are ignored in this example as the process of specifying constraints is described in the following section (see Section 5.2).

Next, the configuration HSQLDBConf is created by replacing the Database virtual component, contained in the configuration BaseConf, with the HSQLDB concrete component. By the same argument as above, $H S Q L D B$ is a specialization of BaseConf.

Finally, the configuration JBossHSQLDBConf is created. This is accomplished by replacing the Database virtual component in JBossConf with $H S Q L D B$, or by replacing the J\&EEAppServer virtual component in HSQLDBConf with JBoss. Either method will produce JBossHSQLDBConf, which is a specialization of both JBossConf and $H S Q L D B C o n f$.

The graphical representations of the specializations created for this instance of the xPetstore system are shown in Figure 5.2. The formal definitions of JBossConf, 
HSQLDBConf, and JBossHSQLDBConf are shown in Table 5.2, Table 5.3 and Table 5.4, respectively.

The second instance of the xPetstore system uses Orion [30] as the J2EE application server and PostgreSQL [31] as the database. The deployable configuration for this instance of the xPetstore system is created by defining a similar set of specializations as described for the previous instance of the system (Figure 5.3).

First the specialization OrionConf is created by replacing the J2EEAppServer virtual component, contained in the configuration BaseConf, with the Orion concrete component. Next, the specialization PostgreSQLConf is created by replacing the Database virtual component, contained in the configuration BaseConf, with the PostgreSQL concrete component. Finally, the specialization OrionPostgreSQLConf is created by replacing Database in OrionConf with PostgreSQL, or by replacing J2EEAppServer in PostgreSQLConf with Orion. OrionPostgreSQLConf is a specialization of both OrionConf and PostgreSQLConf.

The graphical representations of the specializations created for this instance of the xPetstore system are shown in Figure 5.3. The formal definitions of OrionConf, PostgreSQLConf, and OrionPostgreSQLConf are shown in Table 5.5, Table 5.6, and Table 5.7 , respectively.

The specialization scheme of the xPetstore system is shown in Figure 5.4. The specialization scheme includes the base configuration and all of the specializations created to obtain the two deployable configurations described above. The formal definition of the specialization scheme is shown in Table 5.8 .

\subsection{Specifying Constraints}

The discovery of constraints in a system is a continuous process that extends throughout the lifetime of a system, and it requires input from all actors involved in the application assembly process. As different instances of a software product are assembled and different instances of a system are deployed, new constraints will be identified by developers, assemblers, deployers and users. These constraints must be properly specified. 


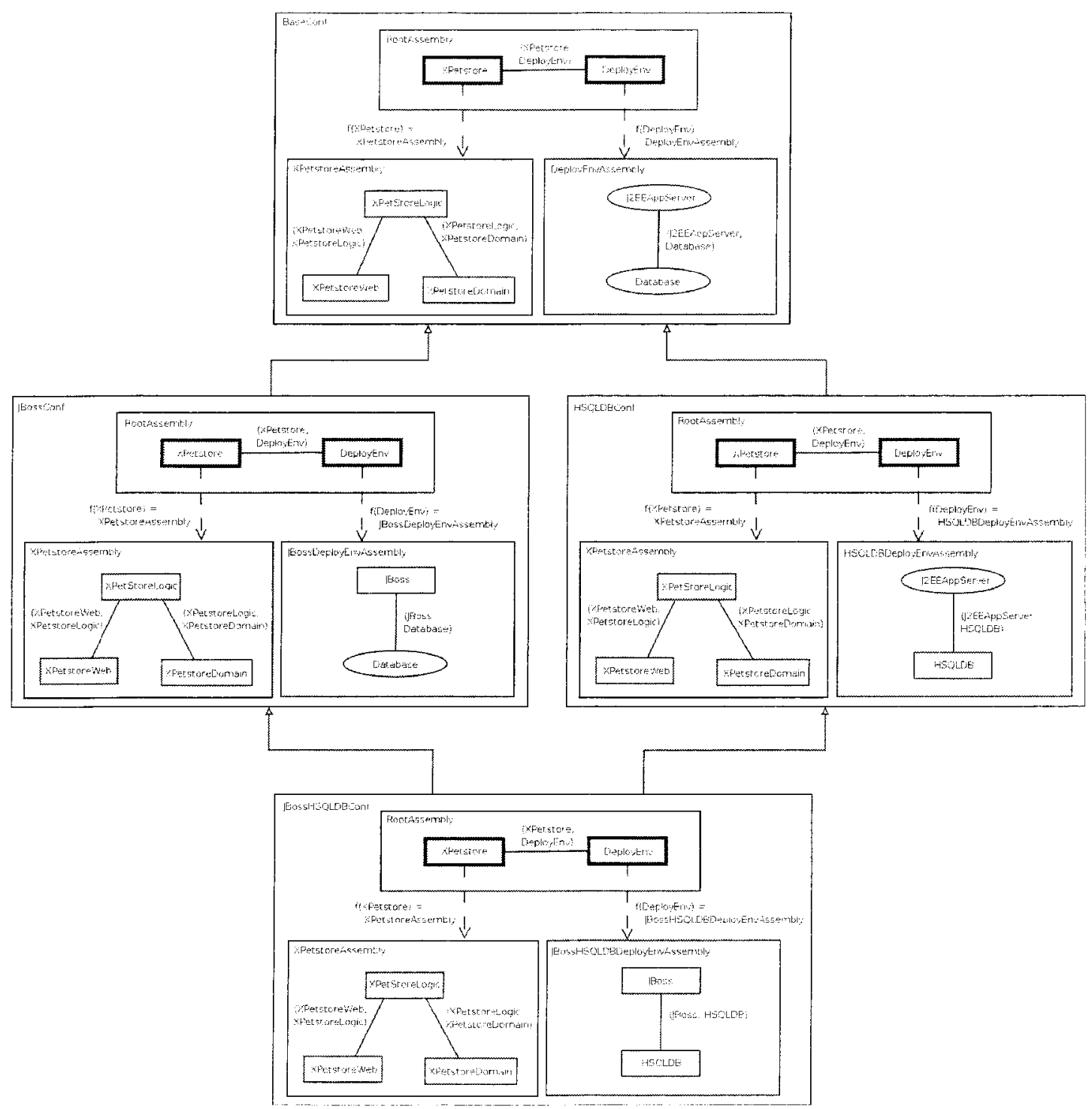

Figure 5.2: The specializations created to obtain a deplovable configuration using JBoss as the J2EE application server and HSQLDB as the database in the xPetstore system. 


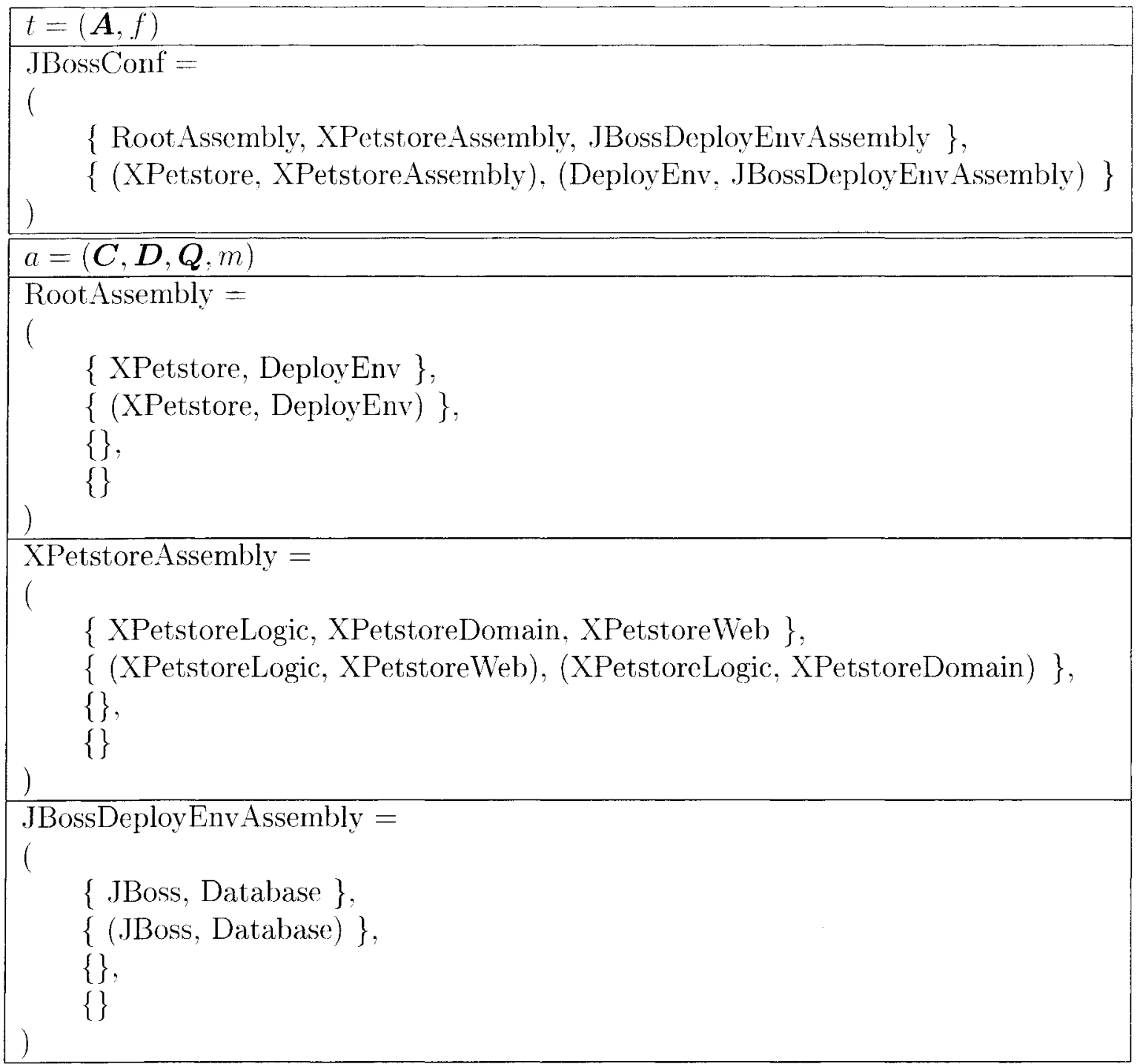

Table 5.2: The formal definition of the JBossConf configuration. 


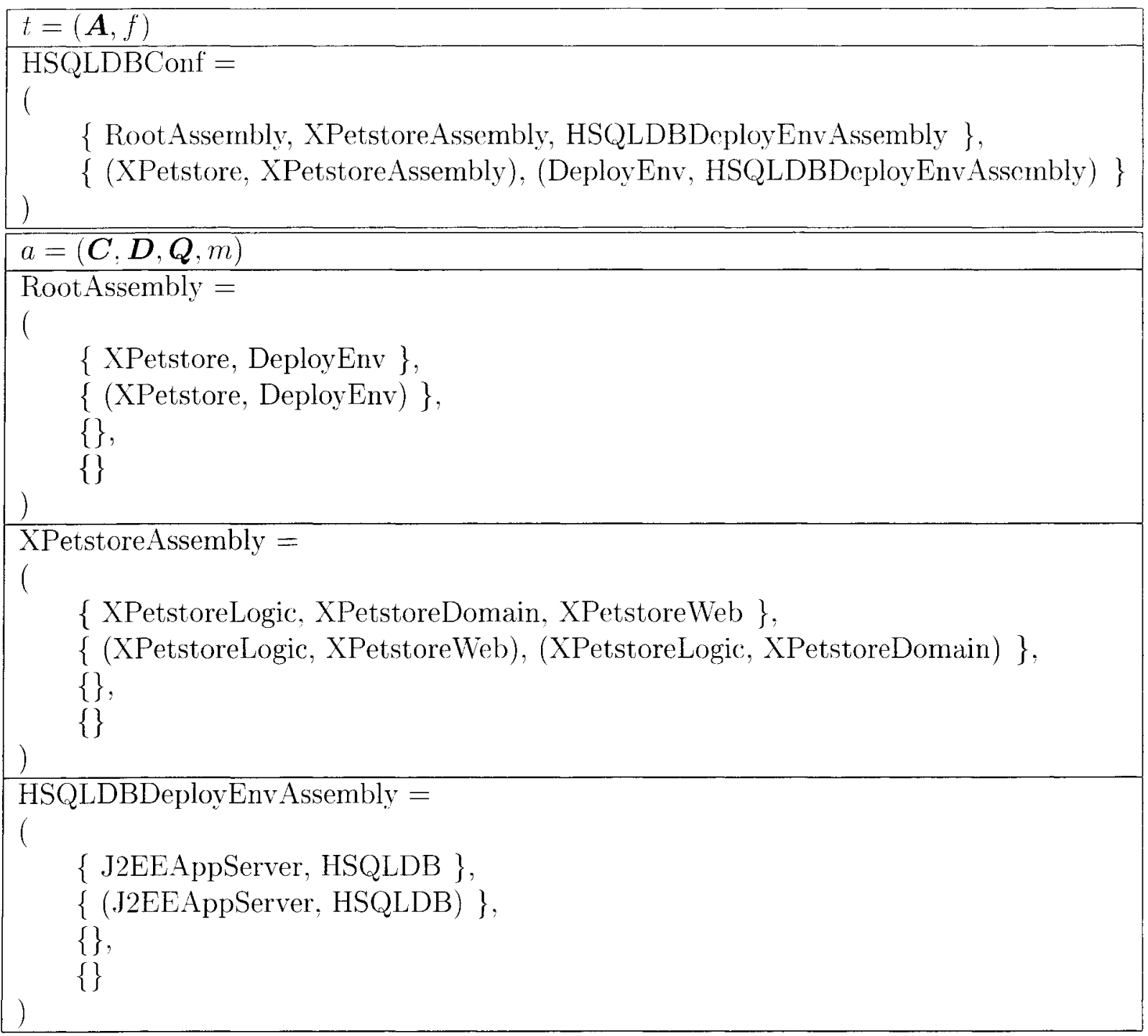

Table 5.3: The formal definition of the HSQLDBConf configuration. 


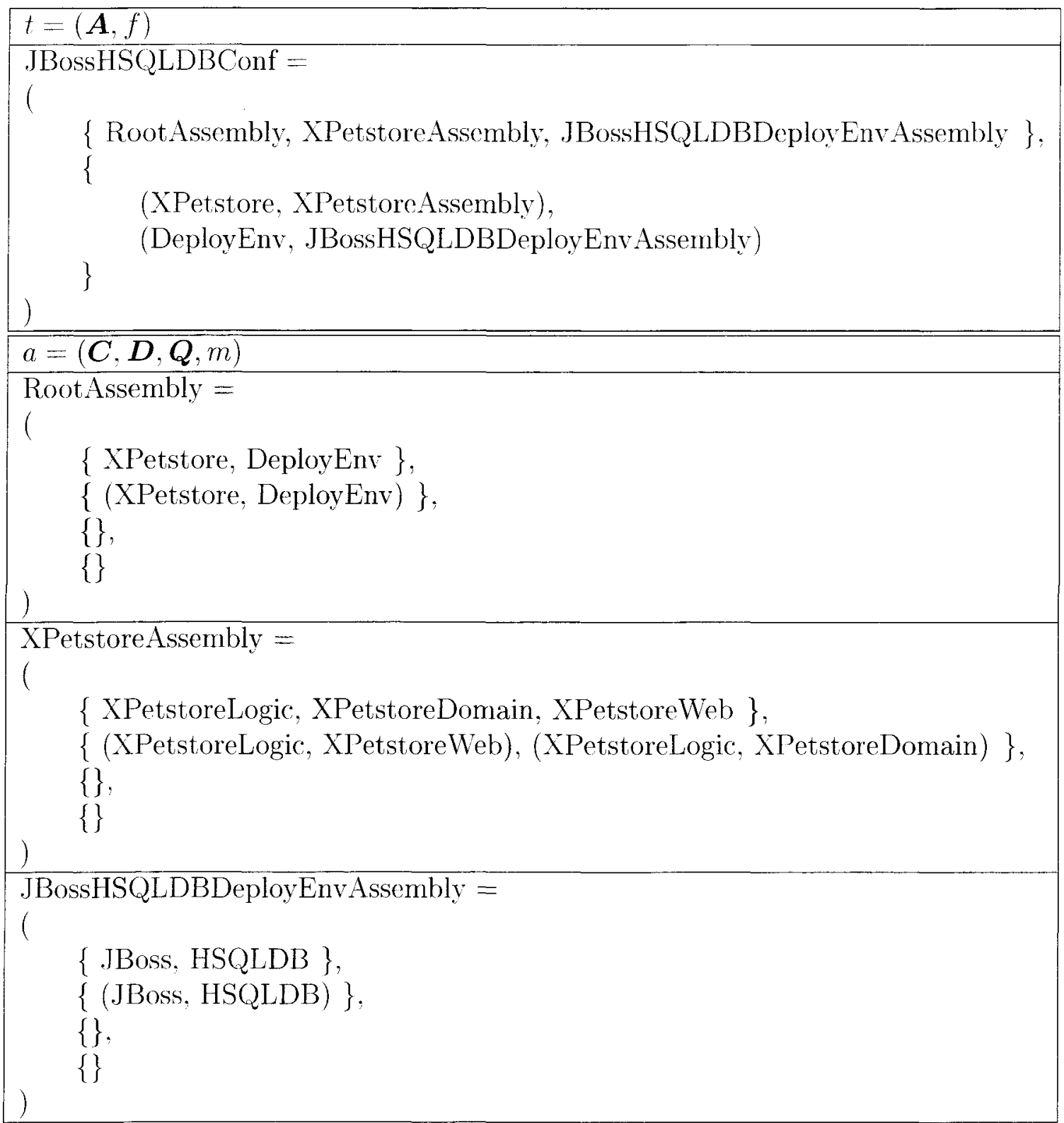

Table 5.4: The formal definition of the JBossHSQLDBConf configuration. 


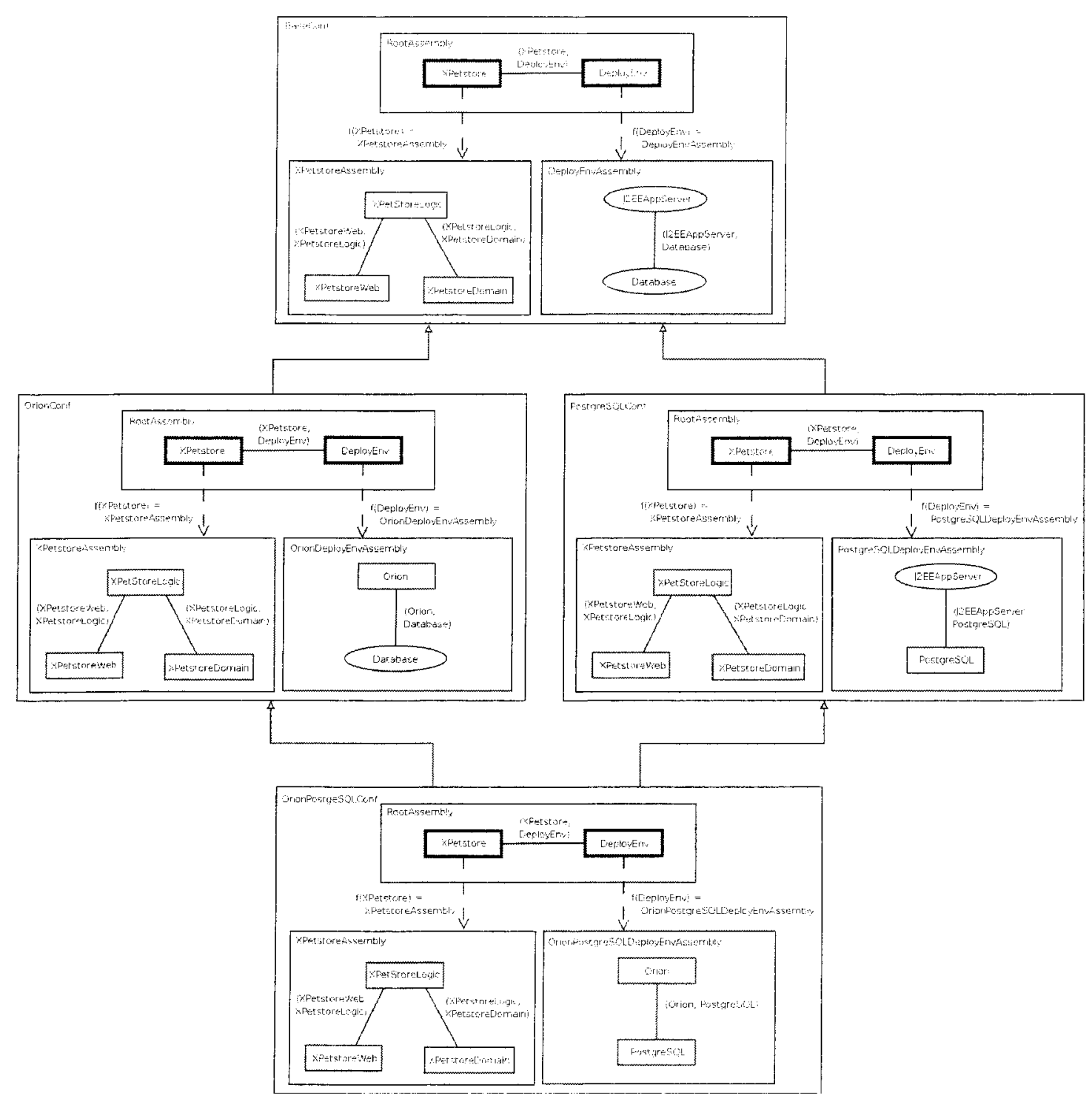

Figure 5.3: The specializations created to obtain a deployable configuration using Orion as the J2EE application server and PostgreSQL as the database in the xPetstore system. 


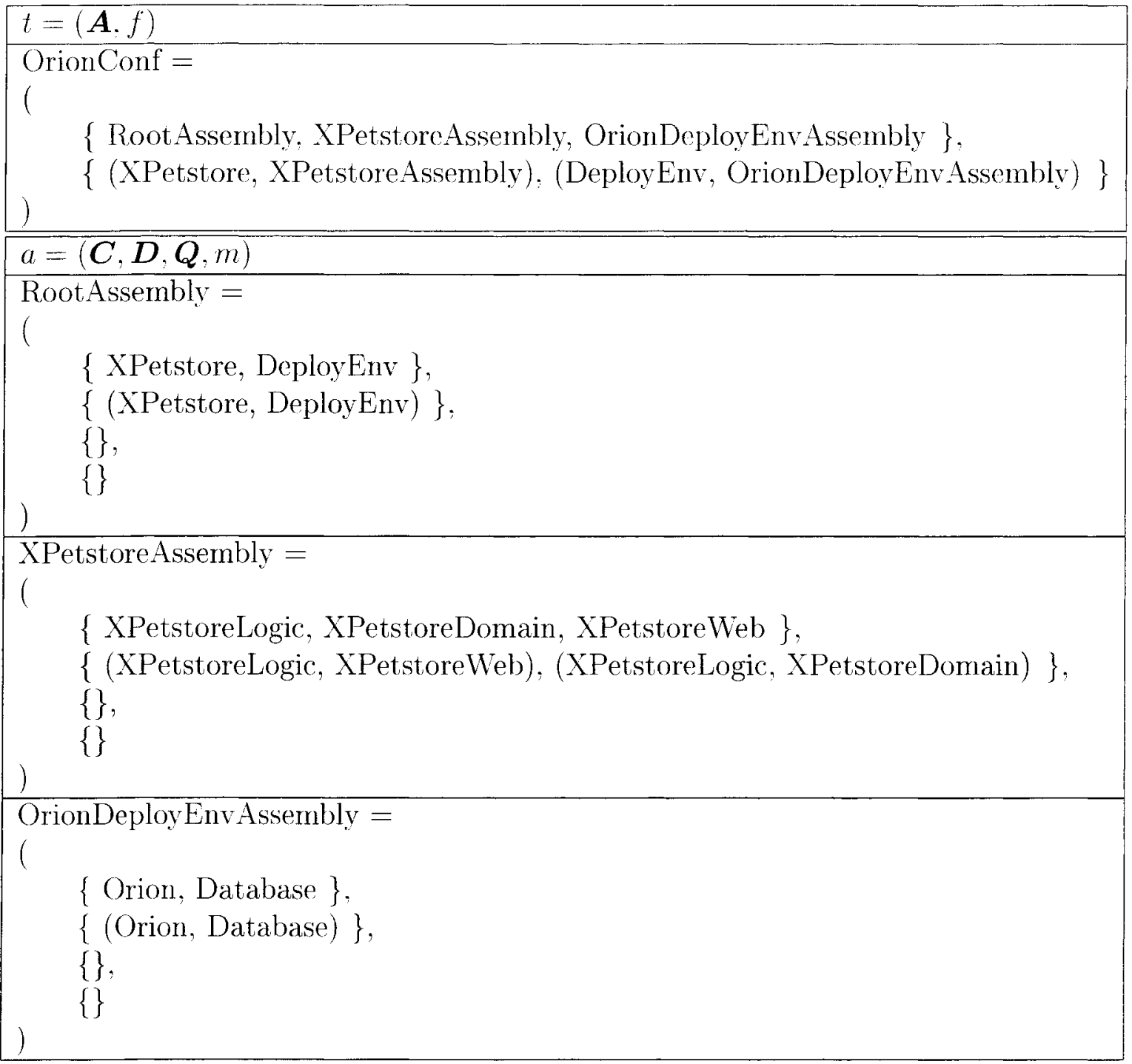

Table 5.5: The formal definition of the OrionConf configuration. 


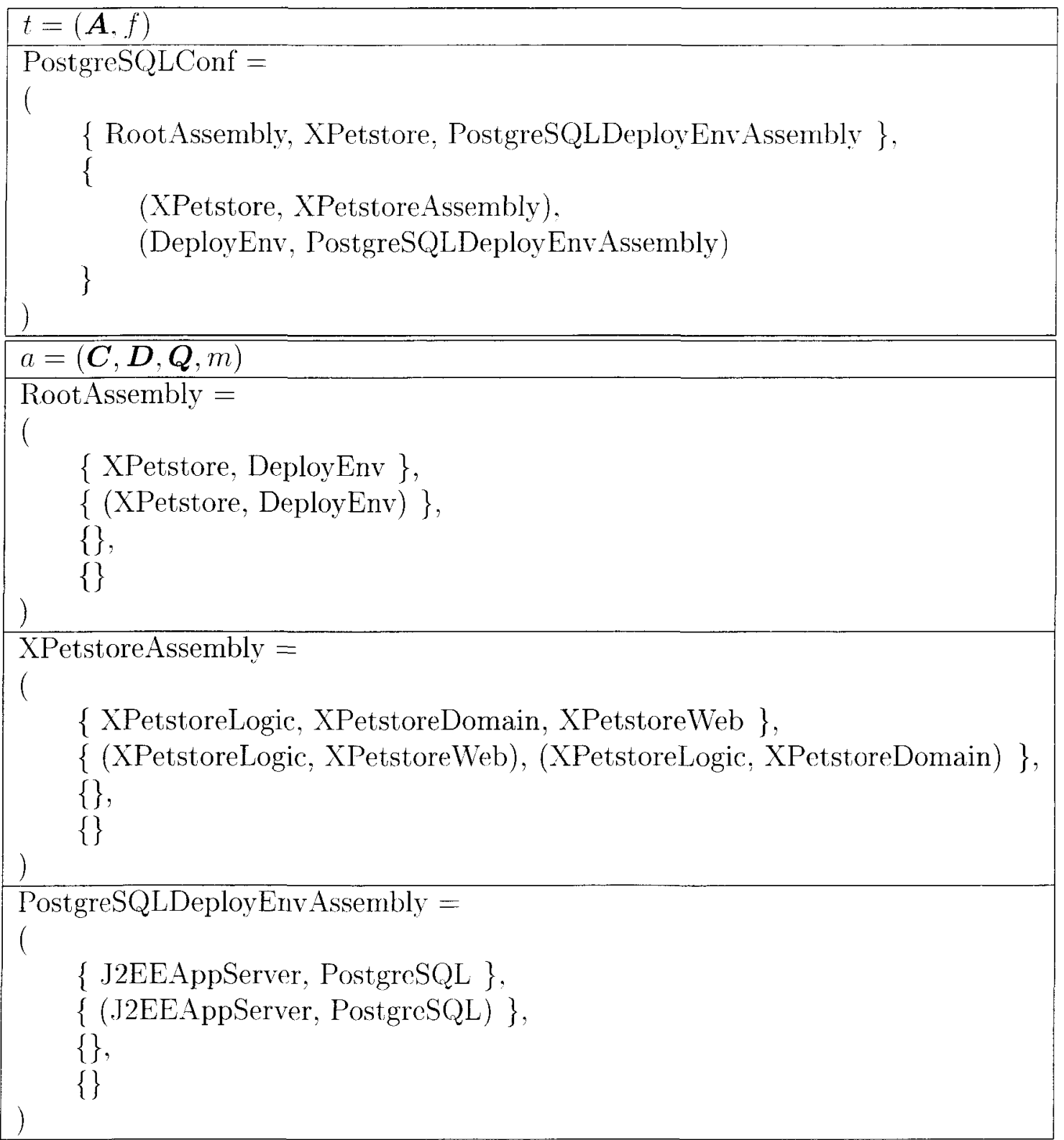

Table 5.6: The formal definition of the PostgreSQLConf configuration. 


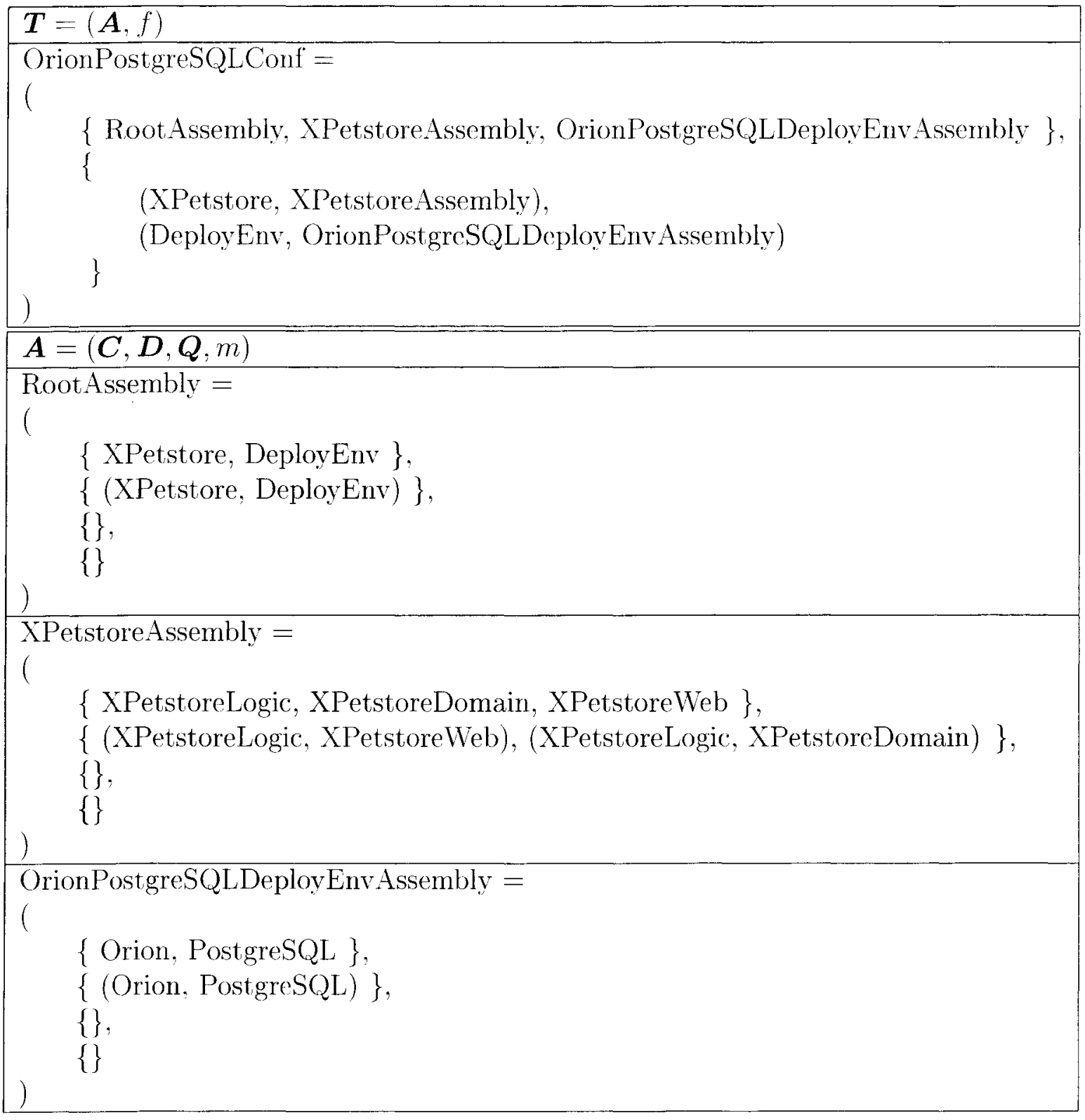

Table 5.7: The formal definition of the OrionPostgreSQLConf configuration. 


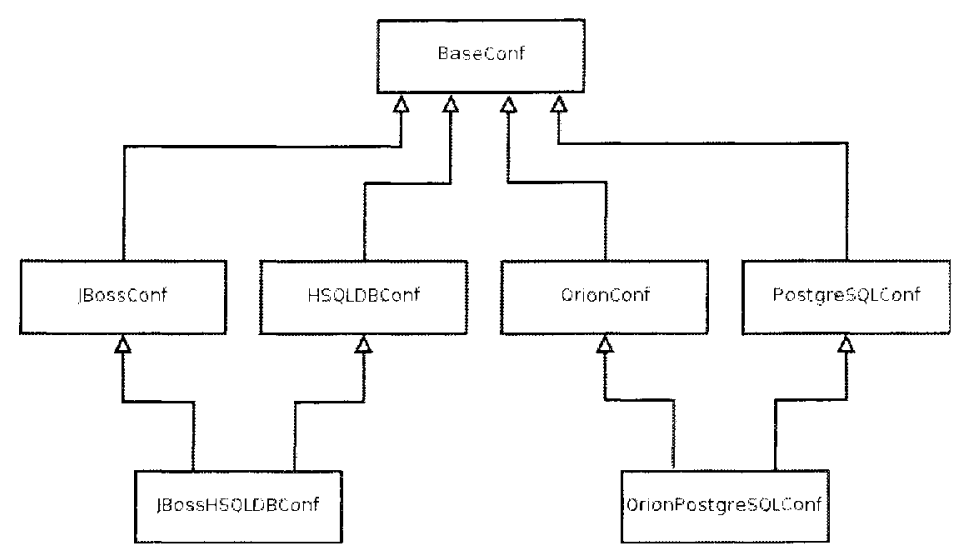

Figure 5.4: The specialization scheme of the xPetstore system.

\begin{tabular}{|ll|}
\hline$s=(\boldsymbol{T}, g)$ \\
\hline XpetstoreSpecScheme $=$ \\
& BaseConf, \\
& JBossConf, \\
& HSQLDBConf, \\
& OrionConf, \\
& PostgreSQLConf, \\
& JBossHSQLDBConf, \\
& OrionPostgreSQLConf \\
\hline & \\
\hline
\end{tabular}

Table 5.8: The formal definition of the specialization scheme of the xPetstore system. 
Using the definition of the system structure, as described in the previous section, it is possible for all actors in the application assembly process to specify constraints within the system. There are two steps involved in specifying a constraint:

1. Declare the constraint

2. Define the constraint

These steps are described in detail in the following sections.

\subsubsection{Declaring Constraints}

Each constraint that is identified must be declared and added to the formal defintion of the software system. Declaring a constraint requires providing an identifier for the constraint which is unique within the formal definition of the system. Also, a textual description must be provided, which explains the context of the constraint and any other general information pertaining to the constraint.

Adding the declared constraint to the formal definition of the system requires inserting it in the appropriate configuration within the specialization scheme. The constraint should be added to the configuration in the specialization scheme in which it is first applicable. That is, it should be added to the configuration containing the most virtual components in which the restriction exists. The reason for this is to prevent having to redeclare the constraint in multiple configurations. If the constraint is added to the first configuration in which it is applicable, then it will be included in each specialization that is derived from that configuration.

The following process could be used to determine the configuration in which to insert the declared constraint:

1. Select a configuration, ConfA, in the specialization scheme in which the constraint is applicable and may be associated with a dependency within an assembly of the configuration

2. If $C o n f A$ is a specialization of another configuration, ConfB, in the specialization scheme, then determine whether the constraint is still applicable in ConfB 
3. If the constraint is applicable in $C o n f B$, then repeat the previous steps using ConfB as the selected configuration (ic. ConfA)

4. Else, the constraint must be added to the configuration ConfA

If a constraint is added to a configuration that has previously been specialized, then the constraint must be propagated to all derived configurations. This is required to maintain the specialization relationships between the configurations within the specialization scheme. By Theorem 3, a specialization must contain all of the constraints that exist within the configuration that was specialized to obtain the specialization.

\subsubsection{Example: Declaring Constraints in the xPetstore Sys- tem}

In order to demonstrate how a constraint is declared, an example constraint, OrderJMSQueue, from the xPetstore system is declared (see Table 5.9). The OrderJM$S Q u e u e$ constraint is associated with the dependency (XPetstore, DeployEnv), which exists between the XPetstore and DeployEnv components.

\begin{tabular}{|l|l|}
\hline Identifier & OrderJMSQueue \\
\hline Description & $\begin{array}{l}\text { The xPetstore system requires that the J2EE } \\
\text { application server, J2EEAppServer, provide a Java } \\
\text { Message Service (JMS) queue for processing orders. }\end{array}$ \\
\hline
\end{tabular}

Table 5.9: The OrderJMSQueue constraint. This constraint is added to the base configuration of the xPetstore system, and it is associated with the (XPetstore, DeployEnv) dependency.

The OrderJMSQueue constraint describes the requirement of a Java Message Service (JMS) message queue for processing customer orders within the xPetstore system. JMS is a service offered by J2EE application servers, thus, the constraint is related to the J2EEAppServer component, which is a subcomponent of the DeployEnv composite component. Since the constraint is applicable no matter which J2EE application server is selected to be used within the xPetstore system, the constraint is added to 
the BaseConf configuration. The formal defintion of the BaseConf configuration is updated to reflect the addition of the OrderJMSQueue constraint, and it is shown in Table 5.10.

The OrderJMSQueue constraint is then propagated to all specializations that have been derived from the BaseConf configuration. These specializations include JBossConf, HSQLDBConf, OrionConf, PostgreSQLConf, JBossHSQLDBConf and OrionPostgreSQLConf. The udpated formal defintions of JBossConf, HSQLDBConf and JBossHSQLDBConf are shown in Table 5.11, Table 5.12, and Table 5.13, respectively. The updated formal definitions of the other specializations have been omitted for brevity.

\subsubsection{Define Constraints}

Declaring a constraint indicates the presence of the constraint within the system; however, a means of verifying the constraint are not explained in the declaration. A constraint must be defined in order to provide the specific details of the constraint. Defining a constraint requires providing a rule set that describes how the constraint may be verified. The rule set explains each step of the process required to check that the condition imposed upon the system by the constraint is satisfied.

A constraint should be defined if it is associated with a dependency between two concrete components. Since concrete components have implementations, it is possible to define a rule set that may be used to verify the constraint.

It is not usual to define a constraint that is associated with a dependency containing a virtual component. The reason for this is that a virtual component has no implementation, thus providing a rule set explaining how to verify the constraint is not possible. For example, a constraint may be declared stating that a JDBC database driver must be installed in order to allow communication between a Java application and a database. However, until it has been decided which database will be used, it is impossible to define the procedure to check for the proper JDBC driver. Once the virtual component is replaced with a concrete (or composite) component, it is possible to define the constraint. If multiple instances of a system are created by 


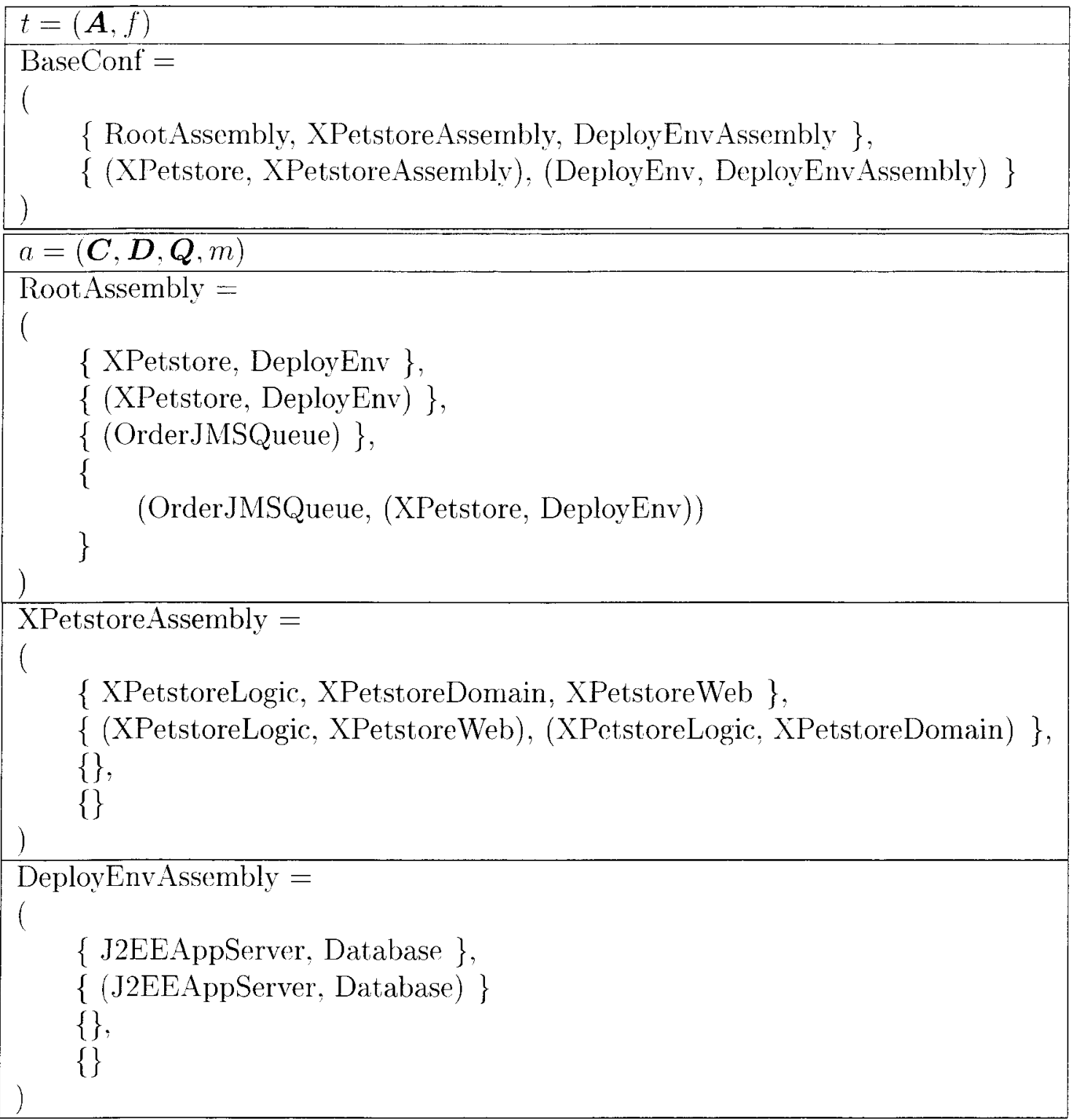

Table 5.10: The formal definition of the BaseConf configuration with the OrderJMSQuene constraint included. 


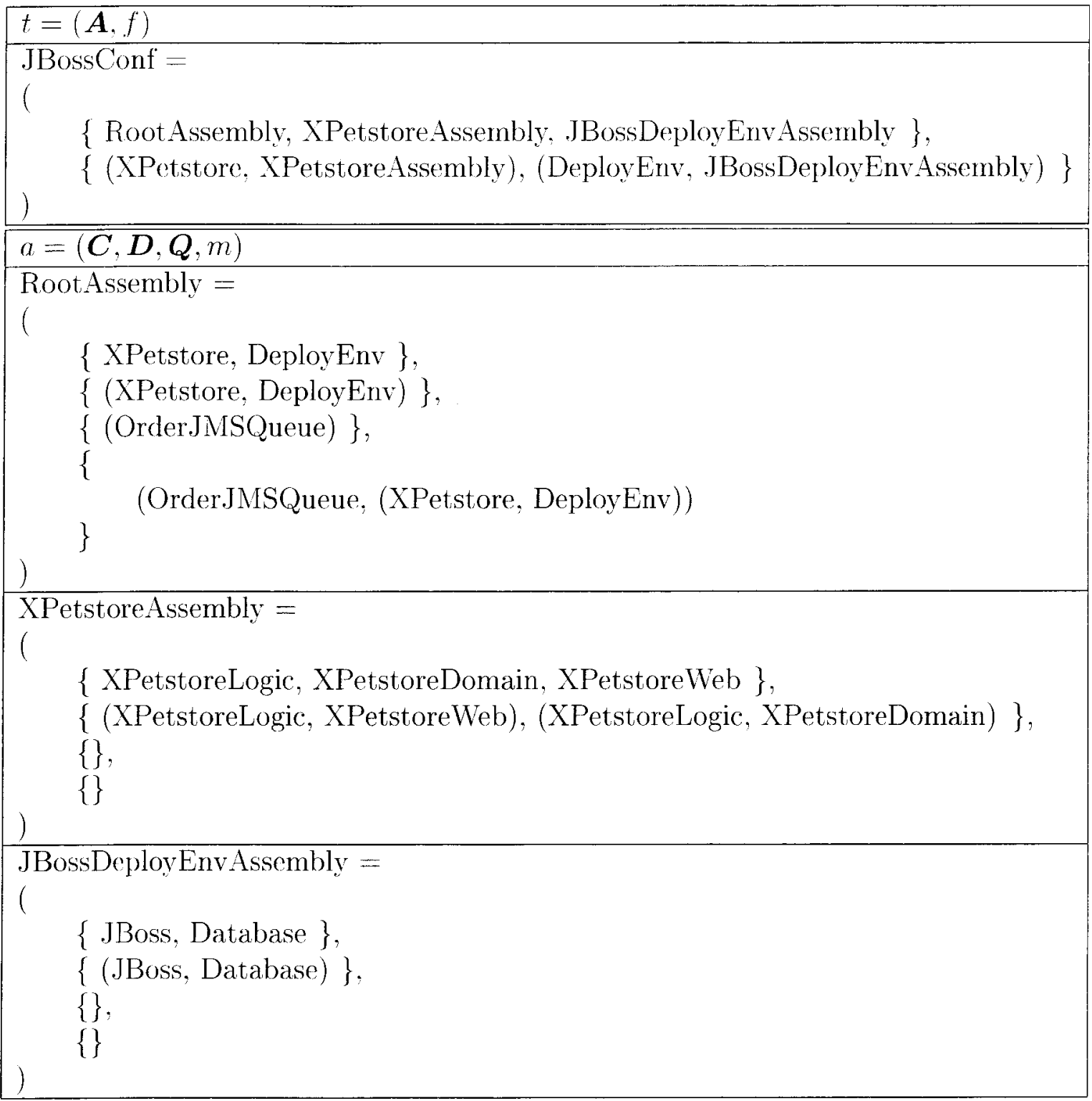

Table 5.11: The formal definition of the JBossConf configuration with the OrderJMSQueue constraint included. 


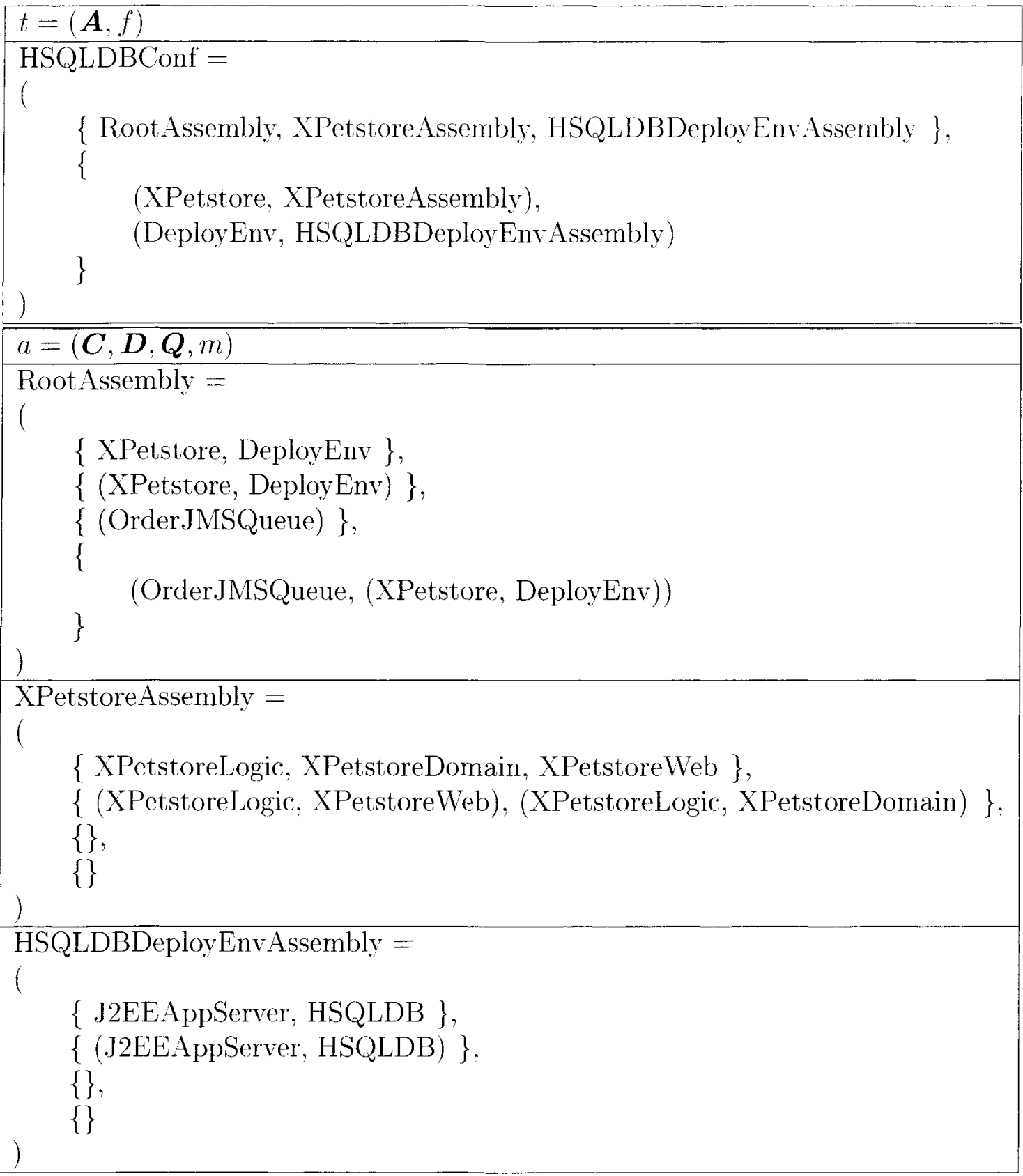

Table 5.12: The formal definition of the HSQLDBConf configuration with the OrderJMSQueue constraint included. 


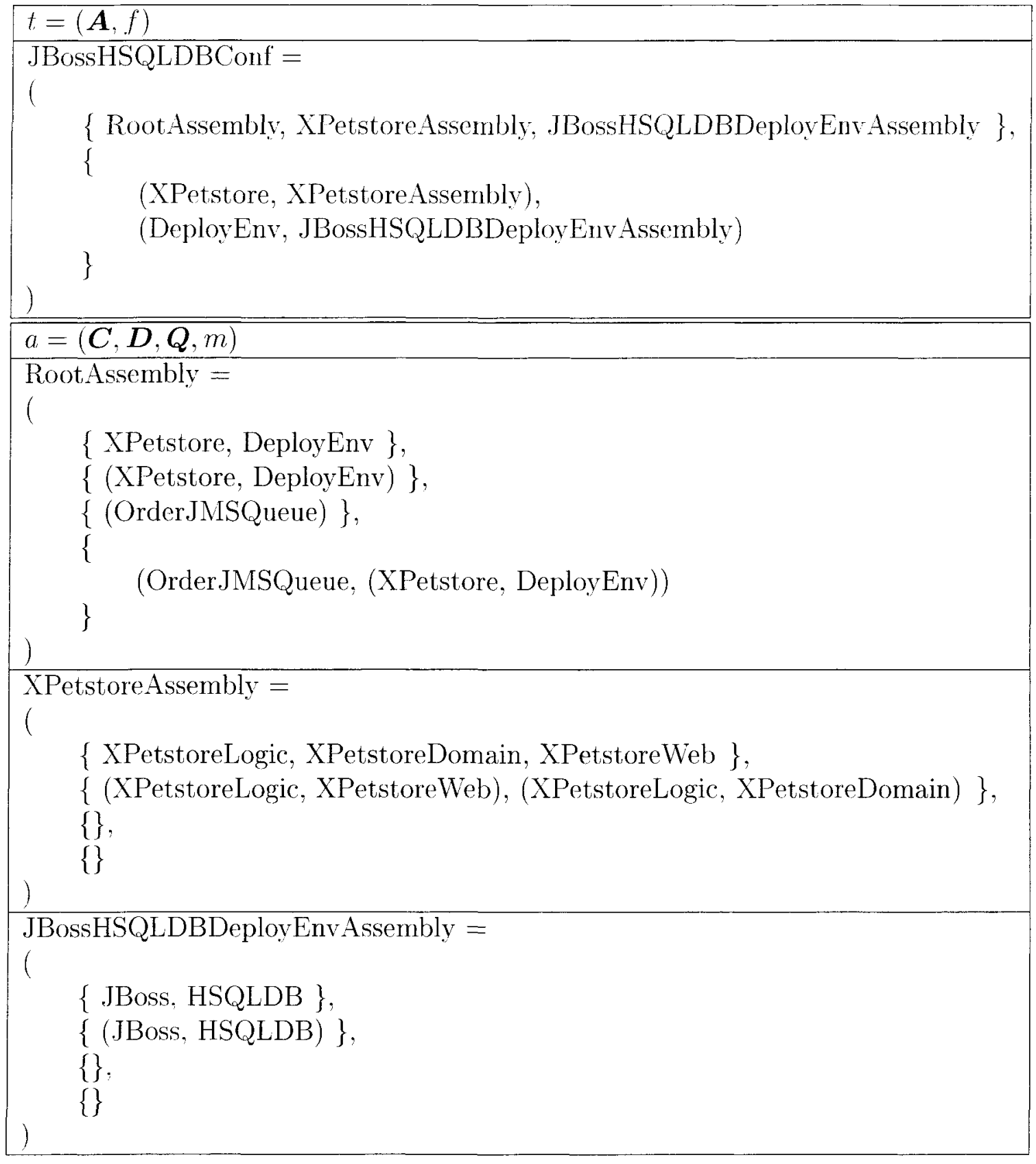

Table 5.13: The formal definition of the JBossHSQLDBConf configuration with the OrderJMSQueue constraint included. 
replacing a virtual component with different concrete/composite components, then the constraints associated with that virtual component will have a different definition for each concrete/composite component used.

It is possible to define a constraint that is associated with a dependency containing a composite component. Since a composite component consists of a set of subcomponents, a constraint associated with that composite component may be related to a particular subcomponent. If the subcomponent is a concrete component, the constraint should be defined; if the subcomponent is a virtual component, it is not possible to define the constraint.

Similar to declaring a constraint, a constraint should be defined in the configuration containing the most virtual components in which the defintion is still applicable. The defintion should also be propagated to all specializations derived from the configuration in which it is first defined. This is not a requirement to maintain the specialization relationships; however, it is beneficial in order to prevent unnecessarily redefining a constraint.

\subsubsection{Example: Defining Constraints in the xPetstore Sys- tem}

In Section 5.2.2, the OrderJMSQueue constraint was declared and added to the BaseConf configuration. Since this constraint is related to the J2EEAppServer component, which is a virtual subcomponent of the DeployEnv composite component, it is not possible to define the constraint in BaseConf.

In the JBossConf configuration, the J2EEAppServer virtual component is replaced with the JBoss concrete component (see Figure 5.2). Thus, it is possible to define the OrderJMSQueue constraint in this configuration. The rule set defined for the OrderJMSQueue constraint when the xPetstore system is deployed using the JBoss application server is shown in Table 5.14.

The rule set shows that the JMS queue must bo properly configured within the jbossmq-destinations-service. $x m l$ configuration file. This file is located in the following directory: 


\section{\$JBOSSHOME/server/\$SERVERNAME/deploy/jms/}

with $\$$ JBOSSHOME representing the path where JBoss is installed, and SSERVERNAME representing the name of the server on which the xPetstore application is deployed. The configuration of the JMS queue is omitted as it is beyond the scope of this example.

This defintion of the OrderJMSQueue should then be propagated to the JBossH$S Q L D B C o n f$ configuration, which is the only specialization of JBossConf in the specialization scheme of the xPetstore system.

\begin{tabular}{|l|l|}
\hline Identifier & OrderJMSQueue \\
\hline Description & $\begin{array}{l}\text { The xPetstore system requires that the J2EE } \\
\text { application server, J2EEAppServer, provide a Java } \\
\text { Message Service (JMS) queue for processing orders. }\end{array}$ \\
\hline Rule Set & $\begin{array}{l}\text { 1. Go to the <jboss_home>/default/<server_name }> \\
\text { directory, where <jboss_home }>\text { is the installation } \\
\text { directory of JBoss, and < server_name }>\text { is the name } \\
\text { of the server on which xPetstore is installed. } \\
\text { 2. Open the } j \text { bossmq-destinations-service.xml file. } \\
\text { 3. Check for order queue configuration }\end{array}$ \\
\hline
\end{tabular}

Table 5.14: The OrderJMSQueue constraint with the rule set defined for when JBoss is selected as the J2EE application server.

In the OrionConf configuration, the J2EEAppServer virtual component is replaced with the Orion concrete component. As a result, it is possible to provide a definition for the OrderJMSQueue constraint that is applicable to the Orion component. The rule set defined for the OrderJMSQueue constraint, in the instance where the $x$ Petstore system is deployed using the Orion application server, is shown in Table 5.15 .

The rule set specifies that the order processing JMS queue must be configured in the jms.xml configuration file. This file is located in the following directory:

\$ORIONHOME/config/ 
with SORIONHOME representing the path where Orion has been installed. The configuration of the JMS queue is not included as it is beyond the scope of this example.

This defintion of the OrderJMSQueue constraint should be propagated to the OrionPostgreSQLConf configuration, which is the only specialization of the OrionConf configuration in the specialization scheme of the xPetstore system.

\begin{tabular}{|l|l|}
\hline Identifier & OrderJMSQueue \\
\hline Description & $\begin{array}{l}\text { The xPetstore system requires that the J2EE } \\
\text { application server, J2EEAppServer, provide a Java } \\
\text { Message Service (JMS) queue for processing orders. }\end{array}$ \\
\hline Rule Set & $\begin{array}{l}\text { 1. Go to the <orion_home>/config directory, where } \\
\text { <orion_home }>\text { is the installation directory of Orion. } \\
\text { 2. Open the jms.xml } \\
\text { 3. Check for order queue configuration }\end{array}$ \\
\hline
\end{tabular}

Table 5.15: The OrderJMSQueue constraint with the rule set defined for when Orion is selected as the J2EE application server.

\subsection{Verifying Configurations}

Once the constraints of a particular configuration have been specified, it is possible to verify the configuration. Verifying a configuration involves manually going through the rule set of each defined constraint within the configuration and ensuring that the constraint is satisfied.

A deplovable configuration should be verified each time that the system has been installed in order to ensure that the system is properly configured and functioning properly.

If the verification process fails, then there exists a constraint within the configuration that is not satisfied. The defined rule set for the unsatisfied constraint should provide cnough information to allow the actor performing the verification to correct the problem. 
If the verification process completes successfully, the deployed system is believed to be functional. However if a deplovable configuration is verified, and it does not function properly, it is likely that there exists an undefined constraint. Once the constraint has been discovered, it must be declared and defined in the manner described in Section 5.2.

\subsection{Sharing System Structure and Constraint In- formation}

It is becoming increasingly common for development teams to be spread across multiple cities and, in some cases, countries. This is typical for open source projects as the developers rarely live in the same location. Also, software products are developed by separate organizations that are spread throughout the world. As a result of this physical separation, it is important to provide some method for information sharing.

The Internet has greatly facilitated this problem of information sharing by providing a common medium that is available to all, regardless of physical location. Using the Internet, organizations may make information available to all parties that make use of the information.

When assembling a software system using products that have been devcloped by organizations across the globe, it is important to be able to access the system structure and constraint information that have been defined for each product. This is necessary in order to prevent having to recreate the system structure and rediscover all of the constraints for a product each time that it is incorporated into a larger product. This may be achieved by linking to the predefined information for a product from within the product being assembled.

Using the formal specification, this can be accomplished by defining a composite component for the product being included. The composite component is then mapped to the root assembly of the appropriate configuration within the specialization scheme of the existing product. This allows for the constraint information to be shared across organizations, and by using a central location for each product and/or system, it 
prevents having to redefine or rediscover system structure and constraint information.

In order for this to be possible, it is a requirement that each organization that produces a software product to use the formal specification and follow the constraint management process defined in this research. If this is the case, then the level of open collaboration amongst the people involved with cach product increases each time the product is incorporated into a larger product. As a result, managing the constraints becomes easier as more people are involved with the discovery and specification of constraints.

\subsection{Dependency Management Tool}

The process of managing dependencies and constraints discussed in the previous sections does not require the use of a tool to perform the steps involved. However, a tool would be useful in facilitating the process. As part of this research, a tool was developed as a proof of concept on how the formal specification may be used for managing dependencies and constraints in a software system.

This section discusses the functionality implemented within the tool, as well as some suggestions for future work that may be done to improve the tool. For a detailed description on using the tool, please refer to Appendix A

\subsubsection{Tool Functionality}

The tool provides the following functionality for assisting in the constraint management process:

- A specialization scheme can be created

- Configurations can be created and specialized

- Constraints can be added, removed and updated

The most important feature of the tool is that it provides support for open collaboration amongst all actors involved with the system in managing the constraints. This was implemented by using a client-server architecture for the tool. 
The server acts as a repository for storing the the system structure and constraint information. This provides a central location for the information that is accessible to all those involved in the constraint managoment process.

The client is developed as a web-based application that provides easy access to the information stored in the repository. The client may be used via a standard web browser, thus constraints may be added, removed and updated easily.

\subsubsection{Tool Improvements}

The tool developed serves only as a proof of concept for using the formal model to manage dependencies and constraints in a software system.

In its current form, creating and specializing configurations requires manually writing an XML file for each configuration. The tool could be enhanced by providing functionality for modeling a configuration graphically, similar to modeling a system using the Unified Modeling Language (UML).

The tool also does not provide support for automatically verifying constraints in a configuration. Currently, the verification rule set of a constraint is expressed as a set of steps that must be manually carried out to determine whether or not the constraint has been satisfied. It should be possible to allow for a script to be provided as the verification rule set. This script may then be executed automatically by the tool to determine if the constraint has been satisfied.

\subsection{Summary}

This chapter described how the formal specification defined in the previous chapter may be used throughout the application assembly process to assist with the management of dependencies and constraints in a system. The three activities in this process are:

1. Define the system structure

2. Specify constraints 


\section{Verify configurations}

The first activity in using the formal model to manage dependencies and constraints is to define the system structure. The system structure may then be used to specify constraints within the system. Finally, configurations may be verified by checking that each defined constraint within the configuration is satisfied. Managing dependencies and constraints is an iterative process, and these activites must be repeated throughout the life cycle of the software system.

A method for sharing the system structure and constraint information amongst organizations was also discussed. This is important when assembling a software product using existing products, as it prevents having to redefine and rediscover this information. The Internet provides the communication channel that makes this sharing possible.

A tool was developed as a proof of concept for using the formal model to manage the dependencies and constraints in a software system. The tool provides the basic functionality of creating, viewing, updating and specializing configurations. It also provides the capability for open collaboration in adding constraints to a system.

The next chapter provides a detailed case study that applies the dependency and constraint management process presented in this chapter to the WitanWeb conference management system. 


\section{Chapter 6}

\section{Case Study: Witan Web}

This chapter presents a case study that applies the models and processes in this thesis to a production software system. The system, called Witan Web, is made up of the assembled Witan Web product and the set of services that are required by the Witan Web product to deploy a functional system.

The dependency and constraint management process developed in Chapter 5 is applied throughout the case study as several deployable configurations of the Witan Web system are defined. All of the constraints within each deployable configuration are also specified.

The first activity of the dependency and constraint management process that must be carried out is to define the structure of the dependency model. This includes defining the base configuration of the system, and then creating a set of specializations until a deployable configuration has been defined.

The base configuration of the system is created by separating the Witan Web product from the set of services that are required to deploy a functional system. The Witan Web product is developed using the J2EE platform, and it is decomposed into a set of Enterprise JavaBean (EJB) modules that contain both session and entity beans, and a web module that contains the web front-end of the product. It also includes external products, such as Struts [37] and Velocity [43], which are produced by different organizations.

The services required by the Witan Web product include: 
- J2EE application server

- Database

- Outgoing email server

- Java Runtime Environment (JRE)

A set of specializations are created to reflect the decisions made while selecting products to be included within the deployed system.

The first set of specializations defined reflect the decision to use different views within the Witan Web product. The Witan Web product uses a Model-View-Controller (MVC) architecture, thus it is possible to use different views within the product without having to modify the model or controller portions of the product. In this case study, two instances of the Witan Web product were assembled using a JavaServer Pages (JSP) [17] based view in one instance and a Velocity [43] based view in the other instance.

Next, a set of specializations are defined to reflect the different application servers used to deploy the Witan Web product. The Witan Web product is a J2EE product, therefore it requires a J2EE application server in order to be deployed. This case study uses two different application servers within the Witan Web system: the JBoss v3 application server and the JBoss v4 application server. A specialization is defined to reflect the selection of each application server.

A set of specializations are defined to reflect the selection of different databases used to deploy the Witan Web product. A database is required by the Witan Web product to persist the data within the system. Two different databases are used within this case study: the MySQL database [27] and the PostgreSQL database [31].

Using these specializations, two different deployable configurations are defined. Each of the deployable configurations contain the following products:

1. The JSP-based view, the JBoss v4 application server and the PostgreSQL database

2. The Velocity-based view, the JBoss v3 application server and the MySQL database 
Both of the deployable configurations use the Sun Java Runtime Environment [14] and the NRC SMTP server to provide the Java Runtime Environment and outgoing email server services, respectively.

For each configuration that is defined, the set of constraints that are applicable to the configuration are specified. This involves declaring and defining the constraints, as necessary.

This chapter is separated into the following sections:

Section 6.1: This section gives a bricf introduction to the Witan Web conference management system, and it describes the features of the system.

Section 6.2: This section describes how the dependency and constraint management tool was used to assist in the definition of the dependency model of the Witan Web system. It also explains how the tool was used by all actors for specifying constraints.

Section 6.3: This section defines the base configuration of the Witan Web system. It presents the hierarchical decomposition of the system by defining the components and dependencies that are included within each assembly of the base configuration. Also, the constraints discovered within the base configuration are specifeid.

Section 6.4: This section describes how multiple instances of the Witan Web software product are assembled by using different views within the product. Two instances of the Witan Web product are assembled using a JSP based view and a Velocity based view. A specialization is defined for each instance, and the constraints associated with each specialization are specified.

Section 6.5: This section describes how different application servers may be used to deploy the Witan Web product. The JBoss v3 and JBoss v4 application servers were selected to be used in deploying the Witan Web product, and a specialization is defined to reflect the decision to include each application server in the Witan Web system. The constraints associated with each specialization are also specified.

Section 6.6: This section describes how the Witan Web system may be deployed with different databases. The MySQL and PostgreSQL databases were selected to be used in deploying the Witan Web product. A specialization is defined to reflect the selection of each database, and the constraints associated with each specialization are 
specified.

Section 6.7: This section presents the two deployable configurations that have been defined within the case study. The first deployable configuration uses the Velocity based view, the JBoss v3 application server and the MvSQL database. The second deployable configuration uses the JSP based view, the JBoss v4 application server and the PostgreSQL database. All of the constraints identified within each deployable configuration are also specified.

Section 6.8: This section summarizes the results of the case study and the different types of constraints that were discovered at each stage of the case study.

\subsection{About Witan Web}

Witan Web is a web-based conference management system that provides support for submitting, reviewing and recommending papers within a conference. It is developed by the Institute for Information Technology of the National Research Council of Canada, and it is available to organizations world wide for organizing conferences.

The Witan Web system allows for authors to register and submit papers for review. Submissions are reviewed by assigned referees, and referee reports are submitted based upon the assessment of the quality of the paper. Committees are created in order to make decisions regarding the papers submitted by the authors and the referee reports submitted by the referees.

Major features of the Witan Web system include:

- Authors and committee members may be registered in the system

- Authors may submit papers

- Multiple tracks for the conference may be created

- Committees and referees may be assigned to a track

- Security policy may be customized to prevent unauthorized users from viewing protected material 
- Customizable report generation facility

\subsection{Dependency and Constraint Management Tool}

The dependency and constraint management tool. which was developed as part of this research, was used throughout the case study to assist with the defintion of the Witan Web dependency model, and the specification of constraints.

First, the base configuration of the Witan Web system was defined within the tool. Then, all of the specializations were created to reflect the different products that were selected to be included within the system.

The dependency model of the system was made available to the actors that have had experience developing, assembling and deploying the Witan Web system. This allowed for constraints to be specified using the tool, and all constraint information was stored in a central location.

\subsection{The Witan Web System Structure}

The first activity of the dependency management process is to define the base configuration of the Witan Web system. This involves identifying all of the functionality required within the system, and assigning the functionality to components.

The decision to use concrete, composite or virtual components is made by evaluating the products available that provide functionality required. The following scenarios outline how this decision may be made:

- If there exists a single product that fulfills the required functionality, and the product may not be decomposed into a set of sub-products, then a concrete component should be used.

- If there are several products available that provide the required functionality, then a virtual component should be used.

- If there exists a single product that fulfills the required functionality, and the 
product may be decomposed into a set of sub-products, then a composite component should be used.

- If there are a set of similar components that may be grouped together to provide. a subset of the functionality within the system. then a composite component may be used to encapsulate functionality offered by the set of components.

Once the components have been defined, the dependencies between the components must be defined. Then, all of the constraints that are applicable in the base configuration must also be specified.

\subsubsection{Overview of the Base Configuration}

The strategy in defining the base configuration of the Witan Web system was to separate the assembled Witan Web software product from the services required to be deployed as part of the Witan Web system. This was done in order to facilitate the creation of specializations as the Assembler is primarily concerned with the components contained within the assembled product, and the Deployer is concerned with the components that represent the required services.

As a result, the root assembly of the Witan Web base configuration defines two composite components (see Section 6.3.2):

- WitanWeb: This represents the assembled Witan Web software product.

- DeployEnv: This represents the deployment environment that contains the services required by the Witan Web software product.

The Witan Web product is a J2EE application, and it makes use of the Enterprise JavaBean (EJB) and Servlet technologies. The Witan Web product is decomposed into a set of J2EE modules (see Section 6.3.3):

- An EJB module consisting of all the entity beans contained within the Witan Web software product (see Section 6.3.5).

- An EJB module consisting of all the session beans that may be accessed only by authorized users of the Witan Web system (see Section 6.3.7). 
- An EJB module consisting of all of the session beans that may be accessed by all users of the Witan Web system (see Section 6.3.9).

- A web module consisting of the Servlet controller of the Witan Web product, and the web front-end view of the product (see Section 6.3.11).

Each module is decomposed into an assembly within the base configuration, with a concrete component defined for each EJB and Servlet contained within the product. The view is represented using a virtual component as different implementations of the view may be used within different deployments. This is a result of the ModelView-Controller (MVC) architecture used within the Witan Web product. Finally, each module contains a composite component that represents the configuration information required within each module. Each J2EE module must contain an XML deployment descriptor that describes the components contained within the module. Also, depending upon the application server used to deploy the product, a set of application server specific deployment descriptors may be required. These deployment descriptors are contained within the configuration components of each module.

The deployment environment is decomposed into a set of components representing the services required by the Witan Web product in order to be deployed as a functional system (see Section 6.3.13). These services include:

- A .J2EE application server in order to deploy the Witan Web product, which is a J2EE application.

- A database server for persisting the data within the Witan Web system.

- A JDBC driver, which is required to allow for communication between the J2EE application server and the database server.

- A Java Runtime Environment (JRE) for executing the Java byte code.

- An outgoing email server for sending email notifications to users of the Witan Web system throughout the duration of the conference management process. 
Since there are multiple products available that provide each of these functionalities, all components within the deployment environment assembly (and the database assembly) are defined as virtual components.

The base configuration of the Witan Web system is shown in Figure 6.1. Each of the assemblies contained in the base configuration is described in the following sections.

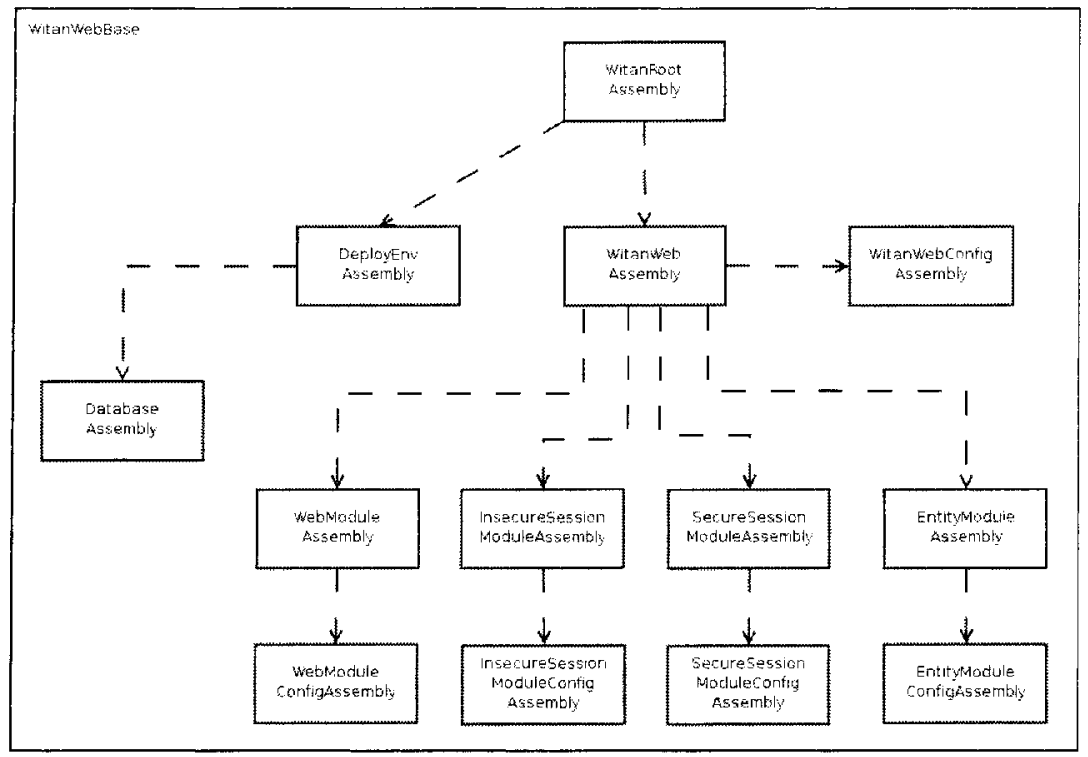

Figure 6.1: The configuration graph of the base configuration for the Witan Web system.

\subsubsection{WitanRootAssembly}

The WitanRootAssembly assembly (Figure 6.2) is the top level assembly of the base configuration, and it represents the start of the hierarchical decomposition of the Witan Web system. The Witan Web system consists of the Witan Web software product, and the products that provide the services required by the Witan Web product in order to be deployed.

The Witan Web product is a J2EE-based application that is assembled from a set 
of modules. Therefore, it requires several external services, such as a J2EE compliant application server and a database, in order to function properly. These services make up the deployment environment.

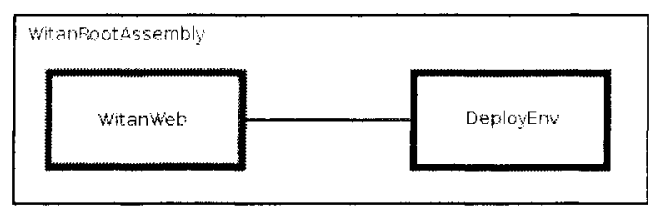

Figure 6.2: The WitanRootAssembly assembly. This is the root assembly of the Witan Web base configuration.

\section{Components}

WitanRoot,Assembly contains the following components (Figure 6.2):

- WitanWeb: This represents the assembled Witan Web conference management software product. This is a composite component as the Witan Web product may be decomposed into a set of separate modules, each of which provides a particular functionality within the product (see Section 6.3.3).

- DeployEnv: This represents the deployment environment of the Witan Web system. This is a composite component as the deployment environment may be decomposed into the individual products that provide the services required by the Witan Web product, such as a J2EE application server, database, and Java runtime (see Section 6.3.13).

\section{Dependencies}

There is a single dependency defined in the WitanRootAssembly assembly:

- (WitanWeb, DeployEnv): This represents the dependency relationship between the Witan Web software product and the environment in which the product will be deployed. Since the Witan Web product is a J2EE application, it requires such services as an application server and database in order to function properly. 


\section{Constraints}

The constraints associated with the (WitanWeb, DeployEnv) dependency include all of the restrictions that are placed upon the deployment environment by the Witan Web product. These include configuration properties that must be set for a particular component, or specific versions of the components that must be used. A sample of the constraints associated with this dependency are shown in Table 6.1.

\begin{tabular}{|l|l|}
\hline Identifier & Ejb20Support \\
\hline Description & $\begin{array}{l}\text { The Witan Web product uses Container Managed Persistence } \\
\text { (CMP) relationships which were first defined in the } \\
\text { Enterprise JavaBean (EJB) } 2.0 \text { specification. Therefore, } \\
\text { the J2EE application server must provide an EJB } \\
\text { container that implements at least the EJB 2.0 } \\
\text { specification. }\end{array}$ \\
\hline \hline Identifier & ConferenceDataSource \\
\hline Description & $\begin{array}{l}\text { The Witan Web product requires that a data source be } \\
\text { configured in the J2EE application server. The data } \\
\text { source is a reference to the database that will be used } \\
\text { to persist the data of the Witan Web product. }\end{array}$ \\
\hline \hline Identifier & OutgoingEmailAccount \\
\hline Description & $\begin{array}{l}\text { The Witan Web product requires an account with } \\
\text { an outgoing email (SMTP) server. This is required for } \\
\text { sending email notifications to users within the system. }\end{array}$ \\
\hline
\end{tabular}

Table 6.1: A sample of the constraints associated with the (Witan Web, DeployEnv) dependency.

\subsubsection{Witan WebAssembly}

The WitanWebAssembly assembly represents the Witan Web software product. The Witan Web product is assembled from a set of J2EE [13] components, including Enterprise JavaBeans (EJBs) and Servlets. The architecture of the product consists of: 
1. Domain model: This is the data model of the Witan Web product, and it is responsible for maintaining and persisting the data within the product. The domain model is implemented as a set of entity beans.

2. Secure business logic: This is the business logic of the Witan Web product that may only be executed by users of the product that have been properly authenticated. The secure business logic is implemented as a set of stateless session beans.

3. Insecure business logic: This is the business logic of the Witan Web product that may be executed by any user of the product. The insecure business logic is implemented as a stateless session bean.

4. Web front-end: This is the user interface of the Witan Web product, which is accessed using a web browser. The controller of the web front-end is implemented using a Serlvet, and the view is implemented using either JavaServer Pages or Velocity.

The flow of control within the Witan Web product proceeds as follows:

1. A user submits a request using the web front-end;

2. The request is processed and passed to either the secure or insecure business logic, depending upon the type of request received;

3. The business logic either submits or retrieves data to/from the domain model and returns the result to the web front-end;

4. The web front-end processes the result and renders the appropriate display for the user

\section{Components}

The Witan WebAssembly assembly consists of the following components (Figure 6.3): 


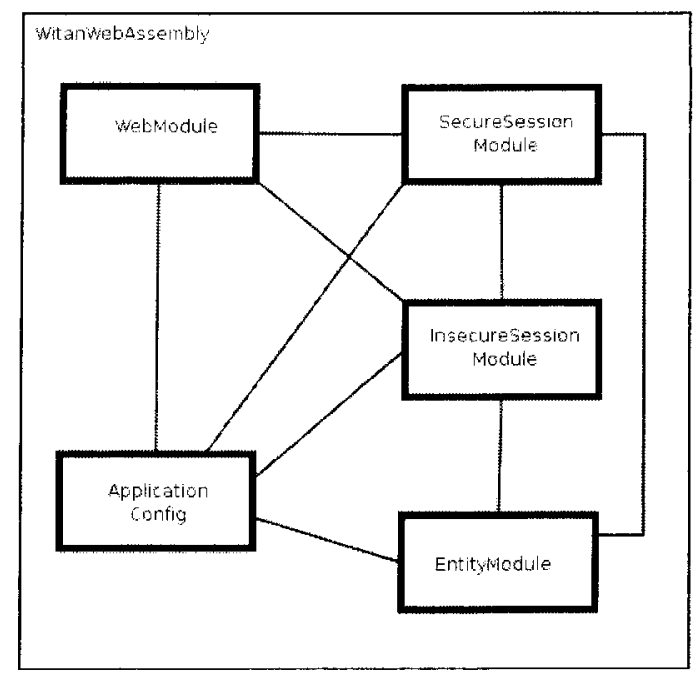

Figure 6.3: The Witan WebAssembly assembly.

- EntityModule: This represents the domain model of the Witan Web product. This is a composite component as it is decomposed into a set of EJB entity beans, each of which represents a single domain object (see Section 6.3.5).

- SecureSessionModule: This represents the secure business logic of the Witan Web product. This is a composite component as it is decomposed into a set of stateless session beans that provide secure operations such as accessing and modifying conference related data (see Section 6.3.7).

- InsecureSessionModule: This represents the insecure business logic of the Witan Web product. This is a composite component as it is decomposed into a set of stateless session beans that provide non-sensitive operations, such as user registration (see Section 6.3.9).

- WebModule: This represents the web front-end of the Witan Web product. This is a composite component as it is decomposed into a Servlet controller and web-based view (see Section 6.3.11). 
- Witan WebConfig: This represents the configuration of the Witan Web product. This is a composite component as it is decomposed into a set of configuration files and XML deployment descriptors (see Section 6.3.4).

\section{Dependencies}

The dependencies contained in WitanWebAssembly include:

- (WebModule, SecureSessionModule): This represents the dependency between the web front-end and the secure business logic of the Witan Web product. This dependency exists as a result of the web front-end receiving a request for a secure operation and passing the request to the secure session module for processing.

- (WebModule, InsecureSessionModule): This represents the dependency between the web front-end and the insecure business logic of the Witan Web product. This dependency exists as a result of the web front-end receiving a request for an insecure operation and passing the request to the insecure session module for processing.

- (WebModule, WitanWebConfig): This represents the dependency between the web front-end and the configuration information of the Witan Web product. This dependency exists as a result of the web front-end needing to be properly configured within the Witan Web product.

- (SecureSessionModule, InsecureSessionModule): This represents the dependency between the secure business logic and the insecure business logic of the Witan Web product. This dependency exists as a result of the insecure session module retrieving the conference status in order to ensure that the registration period is still open.

- (SecureSessionModule, EntityModule): This represents the dependency between the secure business logic and the domain model of the Witan Web product. This dependency exists as a result of the securc session module needing to retrieve and submit data from the domain model. 
- (SecureSessionModule, WitanWebConfig): This represents the dependency bctween the secure business logic and the configuration information of the Witan Web product. This dependency exists as a result of the secure session module needing to be configured within the Witan Web product.

- (InsecureSessionModule, EntityModule): This represents the dependency between the insecure business logic and the domain model of the Witan Web product. This dependency exists as a result of the insecure session module needing to access and modify non-sensitive data in the domain model.

- (InsecureSessionModule, WitanWebConfig): This represents the depedency between the insecure business logic and the configuration information of the Witan Web product. This dependency exists as a result of the insecure session module needing to be configured within the Witan Web product.

- (EntityModule, WitanWebConfig): This represents the dependency between the domain model and the configuration information of the Witan Web product. This dependency exists as a result of the entity module needing to be configured within the Witan Web product.

\section{Constraints}

The constraints associated with the dependencies:

- (WebModule, SecureSessionModule)

- (WebModule, InsecureSessionModule)

- (SecureSessionModule, InsecureSessionModule)

- (SecureSessionModule, EntityModule)

- (InsecureSessionModule, EntityModule)

are all related to ensuring that the beans included in any of the EJB modules (SecureSessionModule, InsecureSessionModule, and EntityModule) are configured so that all modules referencing the beans will function properly. 
For example, the WebModule references the RetrievalEJB session bean located in the SecureSessionModule. Therefore, the (WebModule. SecureSessionModule) dependency has a constraint associated with it stating that the RetrievalEJB bean must be configured in the ejb-jar.xml deployment descriptor. This constraint is shown in Table 6.2.

\begin{tabular}{|l|l|}
\hline Identifier & RetrievalEJBDef \\
\hline Description & $\begin{array}{l}\text { The web front-end of the Witan Web product requires that } \\
\text { the Retrieval session bean be properly defined in the } \\
\text { ejb-jar.xml deployment descriptor of the secure } \\
\text { session module. }\end{array}$ \\
\hline Rule Set & $\begin{array}{l}\text { 1. Open the ejb-jar.xml deployment descriptor of the } \\
\text { secure session module. } \\
\text { 2. Locate the definition of the Retrieval session bean. } \\
\text { 3. Check that the home element is set to: } \\
\text { nrc.witan.session.retrieval.RetrievalHomeRemote } \\
\text { 4. Check that the remote element is set to: } \\
\text { nrc.witan.session.retrieval.RetrievalRemote } \\
\text { 5. Check that the bean-class element is set to: } \\
\text { nrc.witan.session.retrieval.RetrievalBean }\end{array}$ \\
\hline
\end{tabular}

Table 6.2: The RetrievalEJBDef constraint that is associated with the (WebModule, SecureSessionModule) dependency.

The constraints associated with the dependencies:

- (WebModule, WitanWebConfig)

- (SecureSessionModule, WitanWebConfig)

- (InsecureSessionModule, Witan WebConfig)

- (EntityMdoule, WitanWebConfig)

define the restrictions to ensure that each module is specified within the configuration file of the Witan Web product. This involves checking in the application.xml deployment descriptor of the Witan Web enterprise application archive to ensure that the 
file name of the archive for each module is correct. In the case of the web module, a context root must be specified which will be the location at which the web front-end may be accessed using a web browser.

To demonstrate the type of constraint associated with these dependencies, the WebModuleArchiveConfig constraint, which is associated with the (WebModule, WitanWebConfig) dependency, is shown in Table 6.3.

\begin{tabular}{|l|l|}
\hline Identifier & WebModuleArchiveConfig \\
\hline Description & $\begin{array}{l}\text { The web module archive must be specified in the } \\
\text { application.xml deployment descriptor of the Witan } \\
\text { Web product. }\end{array}$ \\
\hline Rule Set & $\begin{array}{l}\text { 1. Open the application.xml deployment descriptor of the } \\
\text { Witan Web enterprise application archive (.ear file). } \\
\text { 2. Locate the definition of the web module. } \\
\text { 3. Check that the web-uri element is set to the name of the } \\
\text { web module archive (.war file). }\end{array}$ \\
\hline
\end{tabular}

Table 6.3: The WebModuleArchiveConfig constraint that is associated with the (WebModule, WitanWebConfig) dependency.

\subsubsection{Witan WebConfigAssembly}

The WitanWebConfigAssembly assembly consists of the configuration and deployment descriptor files that are required by the Witan Web product. Every J2EE enterprise application archive must have an application.xml deployment descriptor. Also, each J2EE application server may require server specific deplovment descriptors to be included to specify non-standard configuration properties for the product. The Witan WebConfigAssembly assembly is shown in Figure 6.4.

\section{Components}

The WitanWebConfigAssembly assembly contains two components (see Figure 6.4:

- ApplicationXmlDD 


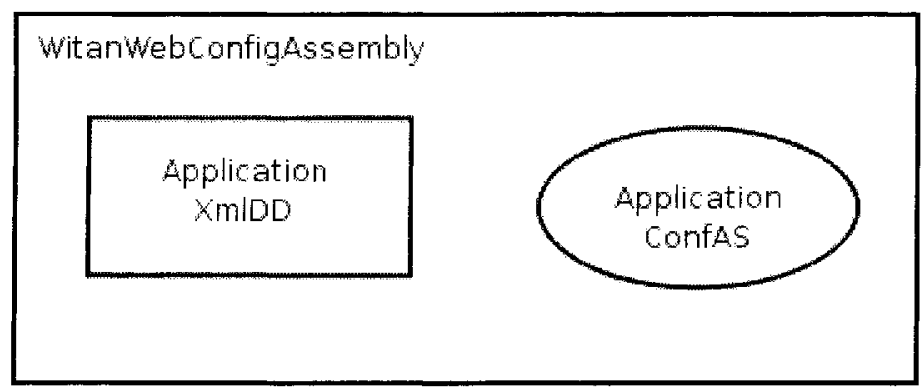

Figure 6.4: The WitanWebConfigAssembly assembly.

- ApplicationConfAS

The ApplicationXmlDD component represents the application.xml deployment descriptor that must be included within all J2EE enterprise archive modules (.ear files). This is defined as a concrete component because there is only a single application.xml deployment descriptor that may be included within the module, and it is not decomposed into a set of sub-components.

The ApplicationConfAS component represents the application server specific configuration that may be required, depending upon which application server is used to deploy the Witan Web product. This is defined as a virtual component because the application server used may differ between organizations deploying the product. Once the application server used to deploy the product has been decided upon, the ApplicationConfAS virtual component may be replaced by the appropriate concrete or composite component.

\section{Dependencies}

There are no dependencies defined within the Witan WebConfigAssembly assembly.

\section{Constraints}

There are no constraints defined within the WitanWebConfigAssembly assembly. 


\subsubsection{EntityModuleAssembly}

The EntityModuleAssembly assembly represents the domain model of the Witan Web product. It consists of a set of container managed entity beans, each of which represents a single data object within the domain model. An entity bean is responsible for persisting data within a database; however, using Container Managed Persistence (CMP), the J2EE application server handles the storage and retrieval of data from the database. The EntityModuleAssembly assembly is shown in Figure 6.5.

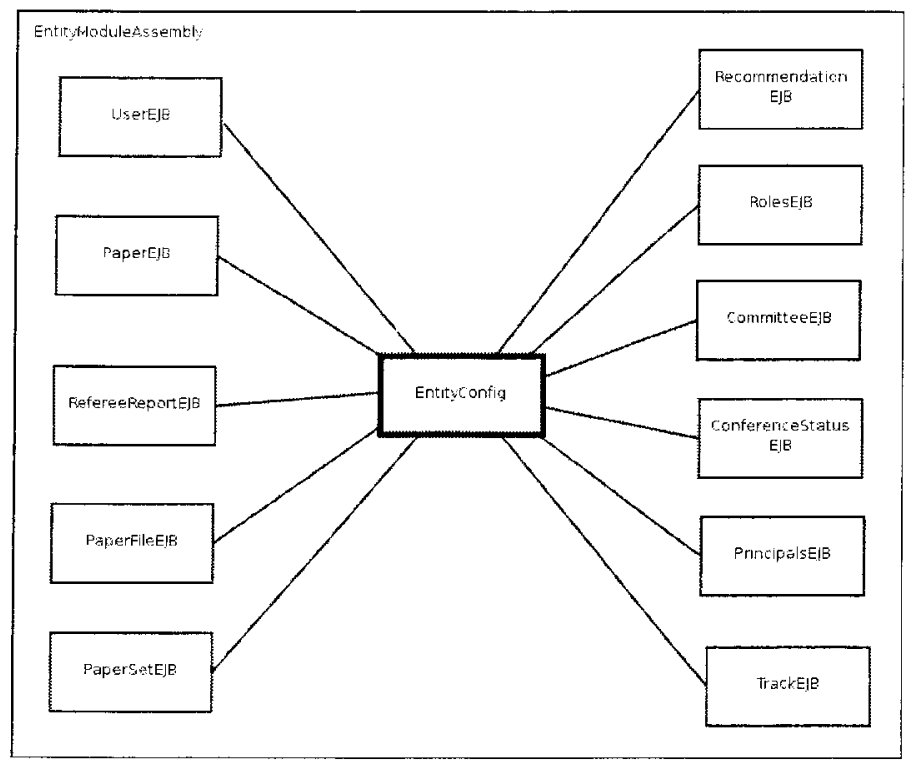

Figure 6.5: The entity module assembly.

\section{Components}

The EntityModuleAssembly assembly contains a single concrete component for each entity bean within the module, and a composite component for the configuration information of the module (Figure 6.5).

The components representing the entity beans include:

- UserEJB 
- PaperEJB

- PaperFileEJB

- PaperSetEJB

- RefereeReportEJB

- CommitteeEJB

- $\operatorname{TrackEJB}$

- RecommendationEJB

- ConferenceStatusEJB

- RolesEJB

- PrincipalsEJB

These are defined as concrete components because there exists a single implementation for each bean, and the beans are not decomposed into a set of sub-components.

The configuration information of the module is represented by the EntityConfig composite component, which is decomposed into a set of configuration files and deployment descriptors that are required by the entity module (see Section 6.3.6).

\section{Dependencies}

The dependencies defined in EntityModuleAssembly include:

- (UserEJB, EntityConfig)

- (PaperEJB, EntityConfig)

- (PaperFileEJB, EntityConfig)

- (PaperSetEJB, EntityConfig)

- (RefereeReportEJB, EntityConfig) 
- (CommitteeEJB, EntityConfig)

- (TrackEJB, EntityConfig)

- (RecommendationEJB, EntityConfig)

- (ConferenceStatusEJB, EntityConfig)

- (RolesEJB, EntityConfig)

- (PrincipalsEJB, EntityConfig)

Each of these dependencies represents the relationship between an entity bean and the configuration of the entity module, as each entity bean must be defined in the ejb-jar.xml deployment descriptor of the entity module.

\section{Constraints}

All of the dependencies in the EntityModuleAssembly assembly pertain to the configuration of each entity bean within the entity module. Thus, the constraints associated with each dependency are similar in that they define the restrictions on the configuration of each bean.

The configuration of an entity bean requires that the local/remote and home interfaces be defined, as well as the bean class for the EJB. Also, each container managed field (CMP field) and query method defined within the bean must be specified. An EJB reference must be specified for each bean that is referenced within the bean being configured.

To demonstrate the types of constraints that exist when configuring an entity bean, scveral constraints associated with the (UserEJB, EntityModuleConfig) dependency are shown in Table 6.4 .

\subsubsection{EntityModuleConfigAssembly}

The EntityModuleConfigAssembly assembly consists of the configuration and deployment descriptor files that are required by the entity module. Every EJB module in a 


\begin{tabular}{|c|c|}
\hline Identifier & UserBeanInterfaceDef \\
\hline Description & $\begin{array}{l}\text { The local and home interfaces, and the bean class of the } \\
\text { User entity bean must be defined in the ejb-jar.xml } \\
\text { deployment descriptor of the entity module. }\end{array}$ \\
\hline Rule Set & $\begin{array}{l}\text { 1. Open the ejb-jar.xml deployment descriptor of the } \\
\text { entity module. } \\
\text { 2. Locate the definition of the User entity bean. } \\
\text { 3. Check that the local element is set to: } \\
\text { nrc.witan.entity.user.UserLocal } \\
\text { 4. Check that the local-home element is set to: } \\
\text { nrc.witan.entity.user. UserHomeLocal } \\
\text { 5. Check that the ejb-class element is set to: } \\
\text { nrc.witan.entity.user. UserBean }\end{array}$ \\
\hline Identifier & UserBeanFindAllQuery \\
\hline Description & $\begin{array}{l}\text { There must be an EJB QL query for the findAll method specified } \\
\text { in the definition of the User bean in the ejb-jar.xml } \\
\text { deployment descriptor of the entity module. }\end{array}$ \\
\hline Rule Set & $\begin{array}{l}\text { 1. Open the ejb-jar.xml deployment descriptor of the entity } \\
\text { module. } \\
\text { 2. Locate the definition of the User entity bean. } \\
\text { 3. Check that there exists a query element containing a } \\
\text { query-method element with the method-name element set } \\
\text { to findAll. } \\
\text { 4. Check that the ejb-ql element contains an EJB QL query that } \\
\text { retrieves all User beans stored in the system. }\end{array}$ \\
\hline Identifier & UserBeanPaperEJBLocalRef \\
\hline Description & $\begin{array}{l}\text { There must be a local EJB reference for the Paper entity bean } \\
\text { specified in the definition of the User entity bean in the } \\
\text { ejb-jar.xml deployment descriptor of the entity module. } \\
\text { The name of the reference must be ejb/PaperLocal. }\end{array}$ \\
\hline Rule Set & $\begin{array}{l}\text { 1. Open the ejb-jar.xml deployment descriptor of the } \\
\text { entity module. } \\
\text { 2. Locate the definition of the User entity bean. } \\
\text { 3. Check that there exists an ejb-local-ref element } \\
\text { with the ejb-ref-name set to ejb/PaperLocal. } \\
\text { 4. Check that the ejb-ref-type element is set to Entity. } \\
\text { 5. Check that the local-home element is set to: } \\
\text { nrc.witan.entity.paper.PaperHomeLocal } \\
\text { 6. Check that the local element is set to: } \\
\text { nrc.witan.entity.paper.PaperLocal }\end{array}$ \\
\hline
\end{tabular}

Table 6.4: A sample of the constraints associated with the (UserEJB, EntityModuleConfig) dependency. 
J2EE application must have an ejb-jar.xml deployment descriptor that defines each of the beans contained within the module. Also, each J2EE application server may require additional deployment descriptors for setting server specific: properties pertaining to the beans included in the EJB module. The EntityModule ConfigAssembly assembly is shown in Figure 6.6.

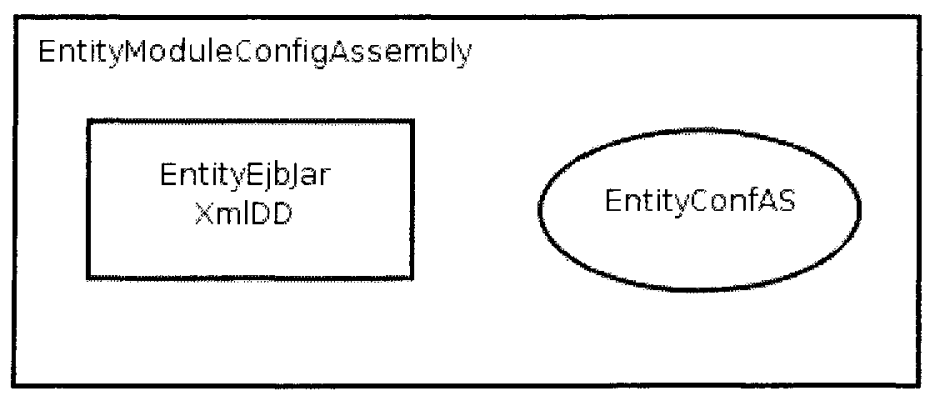

Figure 6.6: The entity configuration assembly.

\section{Components}

The EntityConfigAssembly assembly consists of two components (Figure 6.6):

- EntityConfEjbJarXmlDD

- EntityConfAS

The EntityConfEjbJarXmlDD component represents the ejb-jar.xml deployment descriptor that is required by each J2EE EJB archive (.ear file). This is defined as a concrete component because there exists only a single ejb-jar.xml deployment descriptor that may be used, and it is not decomposed into a set of sub-components.

The EntityConfAS component represents the configuration that is specific to the application server used to deploy the Witan Web product. This is defined as a virtual component because the application server used may differ amongst the organizations deploying the product. Once an application server has been selected, the EntityCon$f A S$ virtual component may be replaced with the appropriate concrete or composite component. 


\section{Dependencies}

There are no dependencies defined in the EntityConfigAssembly assembly.

\section{Constraints}

There are no constraints defined in the EntityConfigAssembly assembly.

\subsubsection{SecureSessionModuleAssembly}

The SecureSessionModuleAssembly assembly represents the secure business logic of the Witan Web product. It consists of a set of stateless session beans that are responsible for retrieving existing data and submitting new or updated data to the domain model. It is also responsible for authenticating and authorizing users within the system in order to provide the necessary security. The SecureSessionModuleAssemby assembly is shown in Figure 6.7 .

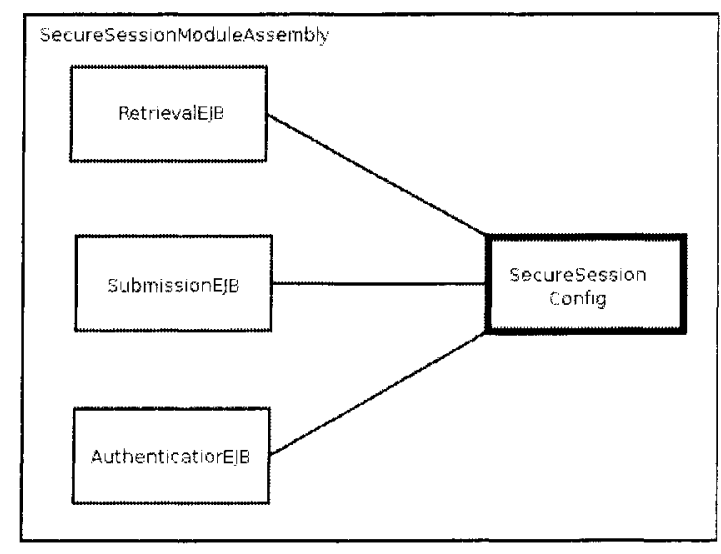

Figure 6.7: The secure session module assembly.

\section{Components}

The SecureSessionModuleAssembly assembly consists of a set of concrete components for each session bean, and a composite component for the configuration information of the secure session module (Figure 6.7). 
The components representing session bcans include:

- SubmissionEJB

- RetrievalEJB

- AuthenticatorEJB

Each of these beans is represented by a concrete component since there exists a single implementation for each session bean, and the beans are not decomposed into a set of sub-components.

The SecureSessionConfig represents the configuration of the secure session module. This is defined as a composite component because it contains the J2EE standard deployment descriptor for the EJB module, and the application server specific configuration files (see Section 6.3.8).

\section{Dependencies}

The dependencies defined in SecureSessionModuleAssembly include:

- (SubmissionEJB, SecureSessionConfig)

- (RetrievalEJB, SecureSessionConfig)

- (AuthenticatorEJB, SecureSessionConfig)

Each of these dependencies represent the relationship between a session bean and the configuration of the secure session module. Each session bean must be defined within the ejb-jar.xml deployment descriptor of the secure session module.

\section{Constraints}

The constraints associated with each dependency in SecureSessionModuleAssembly are similar across all dependencies as they pertain to the configuration of each session bean within the secure session module.

The restrictions that exist in the configuration of each session bean include specifying the local/remote and home interfaces of the bean, as well as the bean class of the 
EJB. The type of the session bean must also be specified. As well, any environment entries and EJB references required by the session bean, must be specified.

To demonstrate the type of constraints related to the configuration of each session bean in the secure session module, a sample of the constraints associated with the (SubmissionEJB, SecureSessionConfig) dependency are shown in Table 6.5.

\subsubsection{SecureSessionModuleConfigAssembly}

The SecureSessionModuleConfigAssembly assembly represents the configuration of the secure session module. Every EJB module in a J2EE application requires an ejbjar.xml deployment descriptor to be included within the module. Also, each J2EE application server may require additional cofiguration and deployment descriptor files in order to specify container specific properties. The SecureSessionModuleConfigAssembly assembly is shown in Figure 6.8.

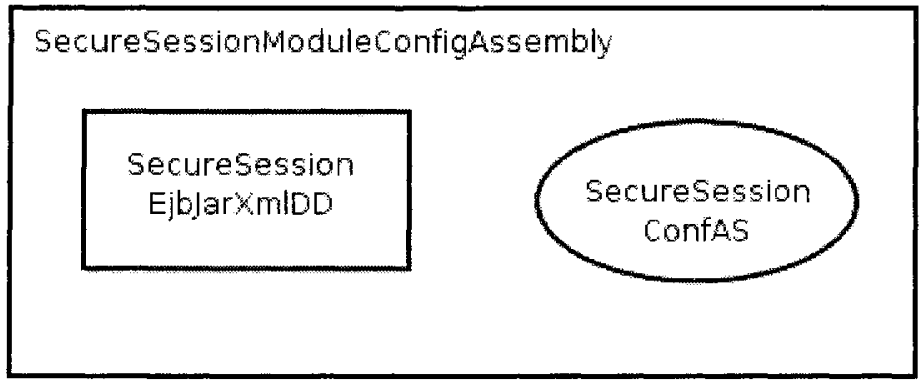

Figure 6.8: The secure session configuration assembly.

\section{Components}

The SecureSessionConfigAssembly assembly contains two components (see Figure $6.8)$ :

- SecureSessionEjbJarXmlDD

- SecureSessionConfAS 


\begin{tabular}{|l|l|}
\hline Identifier & SubmissionBeanInterfaceDef \\
\hline Description & $\begin{array}{l}\text { The remote and home interfaces, and the bean class of the } \\
\text { Submission session bean must be defined in the ejb-jar.xml } \\
\text { deployment descriptor of the secure session module. }\end{array}$ \\
\hline 1. Open the ejb-jar.xml deployment descriptor of the \\
secure session module. \\
2. Locate the definition of the Submission session bean. \\
3. Check that the remote element is set to: \\
nrc.witan.session.submission.SubmissionRemote \\
4. Check that the home element is set to: \\
nrc.witan.session.submission.SubmissionHomeRemote \\
$\begin{array}{l}\text { 5. Check that the ejb-class element is set to: } \\
\text { nrc.witan.session.submission.SubmissionBean }\end{array}$ \\
\hline Description & $\begin{array}{l}\text { SubmissionBeanPaperEJBLocalRef } \\
\text { There must be a local EJB reference for the Paper entity bean } \\
\text { ejb-jar.xml deployment descriptor of the secure session } \\
\text { module. The name of the reference must be ejb/PaperLocal. }\end{array}$ \\
\hline Rule Set & $\begin{array}{l}\text { 1. Open the ejb-jar.xml deployment descriptor of the } \\
\text { secure session module. } \\
\text { 2. Locate the definition of the Submission session bean. } \\
\text { 3. Check that there exists an ejb-local-ref element } \\
\text { with the ejb-ref-name set to ejb/PaperLocal. } \\
\text { 4. Check that the ejb-ref-type element is set to Entity. } \\
\text { 5. Check that the local-home element is set to: } \\
\text { nrc.witan.entity.paper.PaperHomeLocal } \\
\text { nrc.witan.entity.paper.PaperLocal }\end{array}$ \\
\hline
\end{tabular}

Table 6.5: A sample of the constraints associated with the (SubmissionEJB, SecureSessionConfig) dependency. 
The SecureSessionEjbJarXmlDD component represents the ejb-jar.xml deplovment descriptor that is required within each J2EE EJB module (.jar file). This is defined as a concrete component because there exists a single ejb-jar.xml deployment descriptor that may be included within the secure session module, and it is not decomposed into a set of sub-components.

The SecureSessionConfAS component represents the application server specific: configuration information, which may be required depending upon the application server used to deploy the Witan Web software product. This is defined as a virtual component because the application server used by each organization deploying the product may differ. Once an application server has been decided upon, the SecureSessionConfAS virtual component may be replaced with the appropriate concrete or composite component.

\section{Dependencies}

There are no dependencies defined in the SecureSessionConfigAssembly assembly.

\section{Constraints}

There are no constraints defined in the SecureSessionConfigAssembly assembly.

\subsubsection{InsecureSessionModuleAssembly}

The InsecureSessionModuleAssembly assembly represents the insecure business logic of the Witan Web product. It consists of a single stateless session bean that is used for registering new users within a conference. The InsecureSessionModuleAssembly assembly is shown in Figure 6.9.

\section{Components}

The InsecureSessionModuleAssembly assembly consists of a single concrete component for the Registration session bean, and a single composite component for the configuration information of the insecure session module (Figure 6.9). 


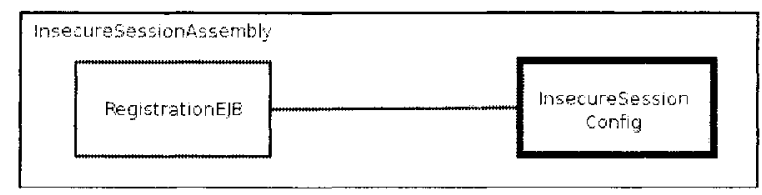

Figure 6.9: The insecure session module assembly.

The RegistrationEJB component represents the Registration session bean. It is defined as a concrete component because there is a single implementation available of the bean, and it is not decomposed into a set of sub-components.

The InsecureSession Config composite component represents the configuration information of the insecure session module. This is defined as a composite component because it is decomposed into a set of sub-components that include the standard J2EE deployment descriptor required by the insecure session module and the application server specific configuration information that may be required, depending upon the application server selected to deploy the Witan Web product (see Section 6.3.10).

\section{Dependencies}

The only dependency defined in InsecureSessionModuleAssembly is:

- (RegistrationEJB, InsecureSessionConfig):

This dependency represents the relationship between the Registration session bean and the configuration of the insecure session module. The Registration bean must be defined within the ejb-jar.xml deployment descriptor of the insecure session module.

\section{Constraints}

The constraints associated with the (RegistrationEJB, InsecureSessionModuleConfig) dependency include all of the restrictions that are required in order for the Registration EJB to be properly defined within the ejb-jar. $x m l$ deployment descriptor.

The configuration of the Regisration session bean requires that the local/remote and home interfaces be specified, as well as the bean class of the EJB. Also, all of the 
EJB references defined within the bean must be specified.

A sample of the constraints associated with the (RegistrationEJB. InsecureSessionModuleConfig) dependency is shown in Table 6.6.

\subsubsection{InsecureSessionModuleConfigAssembly}

The InsecureSessionModuleConfigAssembly represents the configuration of the insecure session module. Every EJB module in a J2EE application requires an ejb-jar.xml deployment descriptor. Also, each application server may require additional configuraiton and deployment descriptor files in order to specify server specific properties. The InsecureSessionModuleConfigAssembly assembly is shown in Figure 6.10.

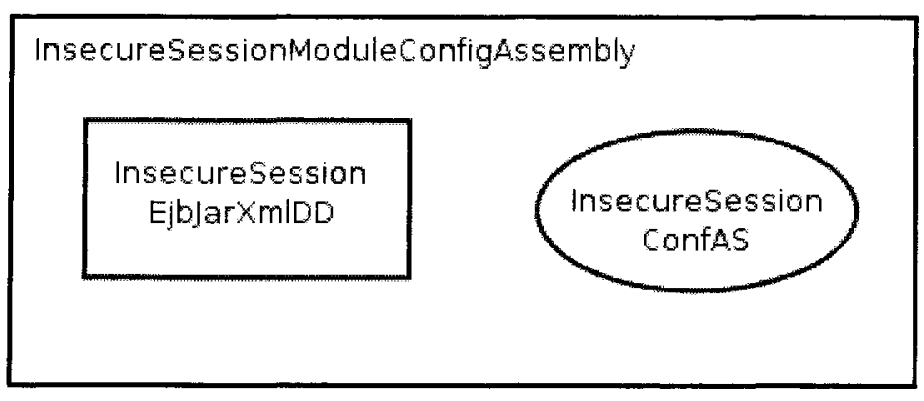

Figure 6.10: The insecure session configuration assembly.

\section{Components}

The InsecureSessionConfigAssembly assembly contains of two components (Figure $6.10)$ :

- InsecureSessionEjbJarXmlDD

- InsecureSession ConfAS

The InsecureSessionEjbJarXmlDD component represents the ejb-jar.xml deployment descriptor that is required within the insecure session module. This is defined as a concrete component because there exists a single ejb-jar. $x m l$ deployment descriptor 


\begin{tabular}{|c|c|}
\hline Identifier & SubmissionBeanInterfaceDef \\
\hline Description & $\begin{array}{l}\text { The remote and home interfaces, and the bean class of the } \\
\text { Registration session bean must be defined in the ejb-jar.xml } \\
\text { deployment descriptor of the insecure session module. }\end{array}$ \\
\hline Rule Set & $\begin{array}{l}\text { 1. Open the ejb-jar.xml deployment descriptor of the } \\
\text { insecure session module. } \\
\text { 2. Locate the definition of the Registration session bean. } \\
\text { 3. Check that the remote element is set to: } \\
\text { nrc.witan.session.submission. RegistrationRemote } \\
\text { 4. Check that the home element is set to: } \\
\text { nrc.witan.session.submission. RegistrationHomeRemote } \\
\text { 5. Check that the ejb-class element is set to: } \\
\text { nrc.witan.session.submission.RegistrationBean }\end{array}$ \\
\hline Identifier & "SubmissionBeanPaperEJBLocalRef \\
\hline Description & $\begin{array}{l}\text { There must be a local EJB reference for the User entity bean } \\
\text { specified in the definition of the Registration session bean in the } \\
\text { ejb-jar.xml deployment descriptor of the insecure session } \\
\text { module. The name of the reference must be ejb/UserLocal. }\end{array}$ \\
\hline Rule Set & $\begin{array}{l}\text { 1. Open the ejb-jar.xml deployment descriptor of the } \\
\text { insecure session module. } \\
\text { 2. Locate the definition of the Registration session bean. } \\
\text { 3. Check that there exists an ejb-local-ref element } \\
\text { with the ejb-ref-name set to ejb/UserLocal. } \\
\text { 4. Check that the ejb-ref-type element is set to Entity. } \\
\text { 5. Check that the local-home element is set to: } \\
\text { nrc.witan.entity.user.UserHomeLocal } \\
\text { 6. Check that the local element is set to: } \\
\text { nrc.witan.entity.user. UserLocal }\end{array}$ \\
\hline
\end{tabular}

Table 6.6: A sample of the constraints associated with the (RegistrationEJB, InsecureSessionModuleConfig) dependency. 
that may be used within the insecure session module, and it is not decomposed into a set of sub-components.

The InsecureSessionConfAS component represents the application server specific configuration information that may be required, depending upon the application server used to deploy the Witan Web product. This is defined as a virtual component because different organizations may use different application servers to deploy the Witan Web product. Once the application server has been selected, then the InsecureSession ConfAS virtual component may be replaced with the appropriate concrete or composite component.

\section{Dependencies}

There are no dependencies defined in the InsecureSessionConfigAssembly assembly.

\section{Constraints}

There are no constraints defined in the InsecureSessionConfigAssembly assembly.

\subsubsection{WebModuleAssembly}

The WebModuleAssembly assembly represents the web front-end of the Witan Web product. The web module consists of a Servlet controller, which uses the Apache Struts framework [37], and a web based view that may be accessed using a web browser. The WebModuleAssembly assembly is shown in Figure 6.11.

\section{Components}

The WebModuleAssembly assembly contains the following components (see Figure $6.11)$ :

- ServletController: This represents the Servlet controller of the wob module. This is responsible for processing all user requests from the web-based view, calling the appropriate session bean in either the secure or insecure session modules, and then returning the appropriate result. 


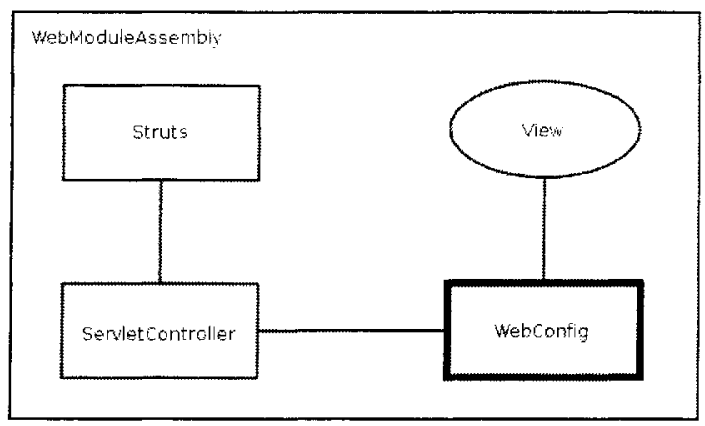

Figure 6.11: The web module assembly.

- View: This represents the web-based view of the web module. This is a virtual component because it is possible to select either a JavaServer Pages (JSP) or Velocity based view to be used within the Witan Web product (see Section 6.4).

- Struts: This represents the Apache Struts framework. It implements the functionality required by the Servlet controller for determining the action to be performed based upon a particular request, as well as the page that should be rendered based upon the result of the action.

- WebConfig: This represents the configuration of the web module. This is defined as a composite component since it is decomposed into a set of subcomponents, which includes the standard J2EE deployment descriptor required within the web module, and the application server specific configuration information that may be required, depending upon the application server used to deploy the Witan Web product (see Section 6.3.12).

\section{Dependencies}

The dependencies defined in WebModuleAssembly include:

- (ServletController, Struts): This dependency represents the relationship between the Servlet controller and the Apache Struts framework. This dependency exists as the controller is built on top of the Servlet framework. 
- (ServletController, WebConfig): This dependency represents the relationship between the Servlet controller and the configuration of the web module. This dependency exists as the controller must be properly configured in order to function properly and integrate with the view.

- (View, WebConfig): This dependency represents the relationship between the view and the configuration of the web module. This dependency exists as the view must be configured properly in order to integrate with the controller.

\section{Constraints}

The constraints associated with the (ServletController, Struts) dependency include all of the restrictions that are required for the Servlet controller to work with the Struts framework. The proper version of the Struts framework must be used, and the JAR file containing the Struts framework must be in a location where it can be loaded by the class loader.

An example of the constraints associated with the (ServletController, Struts) dependency is shown in Table 6.7.

\begin{tabular}{|l|l|}
\hline Identifier & StrutsVersion \\
\hline Description & The Servlet controller requires Struts version 1.1 or greater. \\
\hline Rule Set & 1. Check that the version of the Struts framework is at least 1.1 \\
\hline
\end{tabular}

Table 6.7: A sample of the constraints associated with the (ServletController. Struts) dependency.

The constraints associated with the (ServletController, WebConfig) dependency pertain to the configuration of the Servlet controller within the web module configuration. The configuration settings that must be specified for the Servlet controller include setting all of the actions that are handled by the controller, and the class that is responsible for handling the action requested. As well, the JavaBeans that are related to the HTML forms must be configured, and all exceptions must be configured. 
An example of the constraints associated with the (ServletController, WebConfig) dependency is shown in Table 6.8.

\begin{tabular}{|l|l|}
\hline Identifier & RegisterUserForm \\
\hline Description & $\begin{array}{l}\text { The RegisterUserForm form bean must be configured in the } \\
\text { struts-config.xml configuration file. }\end{array}$ \\
\hline Rule Set & $\begin{array}{l}\text { 1. Open the struts-config.xml configuration file. } \\
\text { 2. Check for the form-bean element with the name attribute set to } \\
\text { RegisterUserForm. } \\
\text { 3. Ensure that the type attribute is is set to } \\
\text { nrc.witan.controller.actionform.UserActionForm }\end{array}$ \\
\hline
\end{tabular}

Table 6.8: A sample of the constraints associated with the (ServletController, WebConfig) dependency.

The constraints associated with the (View, WebConfig) dependency pertain to the configuration of the web view within the web module configuration. The configuration setttings that must be specified for the view include configuring the actions of the HTML forms defined within the view and the page to which control is forwarded upon the successful completion of an action.

A sample of the constraints associated with the (View, WebConfig) dependency is shown in Table 6.9. Since View is a virtual component, the constraint may only be declared at this time; they are defined once the View component is instantiated.

\begin{tabular}{|l|l|}
\hline Identifier & RegistrationAction \\
\hline Description & $\begin{array}{l}\text { The Registration action must be configured in the } \\
\text { struts-config.xml configuration file. }\end{array}$ \\
\hline
\end{tabular}

Table 6.9: A sample of the constraints associated with the (View, WebConfig) dependency. 


\subsubsection{WebModuleConfigAssembly}

The WebModuleConfigAssembly assembly represents the configuration of the web module. Every web module in a J2EE application is required to have a web.xml deployment descriptor, and it is used to define each of the Servlets contained in the wob module. Also, any of the JSP tag libraries that are used within the application must be defined, along with all environment entries required within the wob module.

The web module also consists of a set of configuration files that are required by the Servlet controller, as they are related to the Apache Struts framework.

The WebModuleConfigAssembly assembly is shown in Figure 6.12.

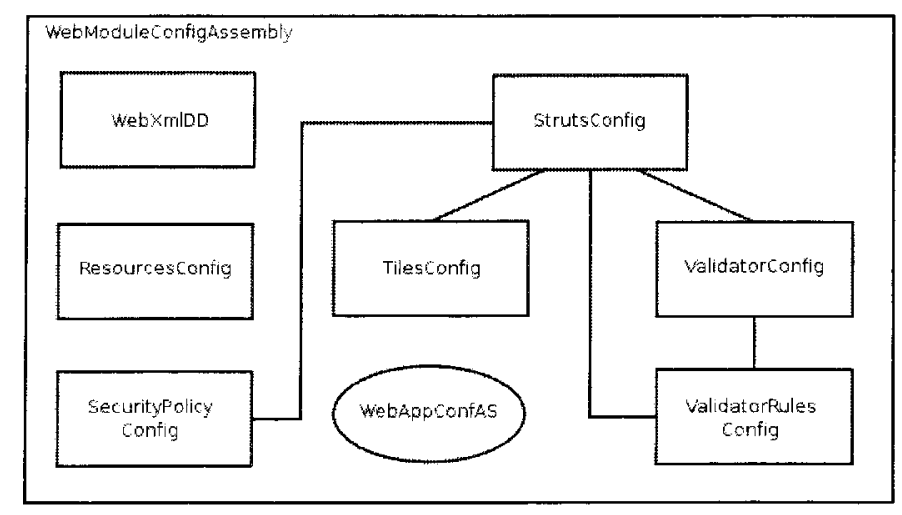

Figure 6.12: The web module configuration assembly.

\section{Components}

The WebModuleConfigAssembly assembly contains the following components (see Figure 6.12):

- WebXmlDD: This represents the web.xml deployment descriptor required by the web module.

- StrutsConfig: This represents the struts-config.xml configuration file that is required by the Struts-based Servlet controller. It specifies the action that should be performed for each type of request that may be made by the user, 
and the page that should be rendered for each possible response to the request. as well as the form beans that are defined within the Servlet controller. It also defines any plug-ins that are used within the Servlet controller.

- TilesConfig: This represents the tiles-defs.xml configuration file that is required by the Struts-based Servlet controller. It specifies the set of layouts that are used within the web view.

- ValidatorConfig: This represensts the validator.xml configuration file that is required by the Struts-based Servlet controller. It specifies the validation procedures that must be performed for each form within the web view.

- ValidatorRulesConfig: This represents the validator-rules.xml configuration file. It specifies a set of validation procedures that may be used to determine whether or not the information entered into a form is valid.

- ResourcesConfig: This represents the ApplicationResources.properties file. It specifies a resource bundle that contains the proper translation of all messages used within the web view.

- SecurityPolicyConfig: This represents the securityPolicies.xml configuration file. It specifies the security roles that are defined within the web module, as well as the access privileges of each role.

- WebAppConfAS: This represents the configuration and deployment descriptor files that are required by the application server used to deploy the Witan Web product. This is defined as a virtual component because the application server used may differ amongst the organizations deploying the Witan Web product. Once the application server has been selected, the WebAppConfAS virtual component may be replaced with the appropriate concrete or composite component.

\section{Dependencies}

The dependencies defined in the WebConfigAssembly assembly include: 
- (StrutsConfig, ValidatorConfig): This dependency represents the relationship between the Struts configuration file and the validator configuration. This dependency exists as the validator must be configured as a plugin within the Struts configuration file.

- (StrutsConfig, ValidatorRulesConfig): This dependency represents the relationship between the Struts configuration and the validator rules configuration. This dependency exists as the validator rules must be configured as a plugin within the Struts configuration file.

- (StrutsConfig, TilesConfig): This dependency represents the relationship between the Struts configuration file and the Tiles configuration. This dependency exists as Tiles must be configured as a plugin within the Struts configuration file.

- (StrutsConfig, SecurityPolicyConfig): This dependency represents the relationship between the Struts configuration file and the security policy configuration. This dependency exists as the security policy must be configured as a plugin within the Struts configuration file.

- (ValidatorConfig, ValidatorRulesConfig): This dependency represents the relationship between the validator configuration file and the validator rules configuration file. This dependency exists as the validation rules required by the validator must be defined within the validator rules configuration file.

\section{Constraints}

The constraints associated with the (StrutsConfig, ValidatorConfig). (StrutsConfig, ValidatorRulesConfig), (StrutsConfig, TilesConfig) and (StrutsConfig, SecurityPolicyConfig) dependencies include the configuration settings that are required for the Validator, Tiles and security policy plugins to be used within the Struts framework.

A sample of the constraints associated with these dependencies are shown in Table 6.10 . 


\begin{tabular}{|l|l|}
\hline Identifier & ValidatorPluginConfig \\
\hline Description & $\begin{array}{l}\text { The Validator must be configured as a plugin within the } \\
\text { struts-config.xml configuration file. }\end{array}$ \\
\hline \hline Identifier & $\begin{array}{l}\text { 1. Open the struts-config.xml configuration file. } \\
\text { 2. Check for the plug-in element with the classNarne attribute set to } \\
\text { org.apache.struts.validator.ValidatorPlugin } \\
\text { 3. Ensure that there is a set-property element specified with the } \\
\text { property attribute set to pathnames and the value attribute } \\
\text { contains the location of the validator.xml configuration file. }\end{array}$ \\
\hline Rescription & $\begin{array}{l}\text { TilesPluginConfig } \\
\text { configuration file. }\end{array}$ \\
$\begin{array}{l}\text { 1. Open the struts-config.xml configuration file. } \\
\text { 2. Check for the plug-in element with the className attribute set to } \\
\text { org.apache.struts.tiles. TilesPlugin } \\
\text { 3. Ensure that there is a set-property element specified with the } \\
\text { property attribute set to definitions-file and the value attribute } \\
\text { set to the location of the tiles-defs.xml configuration file. } \\
\text { 4. Ensure that there is a set-property element specified with the } \\
\text { property attribute set to definitions-debug and the value attribute } \\
\text { set to 0. } \\
\text { 5. Ensure that there is a set-property element specified with the } \\
\text { property attribute set to definitions-parser-details and the value } \\
\text { attribute set to 0. } \\
\text { 6. Ensure that there is a set-property clement specified with the } \\
\text { property attribute set to definitions-parser-validate and the value } \\
\text { attribute set to true. }\end{array}$ \\
\hline
\end{tabular}

Table 6.10: A sample of the constraints associated with the (StrutsConfig, ValidatorConfig), (StrutsConfig, ValidatorRulesConfig), (StrutsConfig, TilesConfig) and (StrutsConfig, SecurityPolicyConfig) dependencies. 
The constraints associated with the (ValidatorConfig. ValidatorRulesConfig) dependency include the configuration of all of the rules that arc used within the validator. The validator requires a set of rules to be defined in order to determine whether or not a form contains valid information for submission.

A sample of the constraints associated with the (ValidatorConfig. ValidatorRulesConfig) dependency is shown in Table 6.11.

\begin{tabular}{|l|l|}
\hline Identifier & RequiredRule \\
\hline Description & $\begin{array}{l}\text { There must be a rule defined in the validator-rules.xml } \\
\text { configuration file for determining whether or not all of the } \\
\text { required fields in a form have been filled in by the user. }\end{array}$ \\
\hline $\begin{array}{l}\text { Rule Set } \\
\text { 2. Open the validator-rules.xml configuration file. } \\
\text { required } \\
\text { 3. Ensure that the className attribute is set to } \\
\text { org.apache.struts.validator.FieldsCheck } \\
\text { 4. Ensure that the method attribute is set to validateRequired. } \\
\text { 5. Ensure that the parameters attribute contains the following: } \\
\text { java.lang.Object } \\
\text { org.apache.commons.validator.ValidatorAction } \\
\text { org.apache.commons.validator.Field } \\
\text { org.apache.struts.action.ActionErrors } \\
\text { javax.servlet.http.HttpServletRequest } \\
\text { 6. Ensure that the msg attribute is set to errors.required. } \\
\text { 7. Ensure that the javascript element contains a JavaScript function } \\
\text { named validateRequired that takes a form as a parameter and checks } \\
\text { to make sure that all fields that are required within the form have } \\
\text { a user defined value specified. }\end{array}$ \\
\hline
\end{tabular}

Table 6.11: A sample of the constraints associated with the (Validator Config, ValidatorRulesConfig) dependency.

\subsubsection{DeployEnvAssembly}

The DeployEnvAssembly assembly represents the deployment environment of the Witan Web system. The deployment environment consists of all of the services required 
by the Witan Web product, which include:

- A J2EE application scrver, which provides the standard J2EE services required by the Witan Web product.

- A database for persisting the data within the system.

- An outgoing cmail server for sending email notifications to the users of the system throughout the conference management process.

- A Java Runtime Environment (JRE) for executing the Java byte code of the application server and Witan Web product.

The DeployEnvAssembly assembly is shown in Figure 6.13.

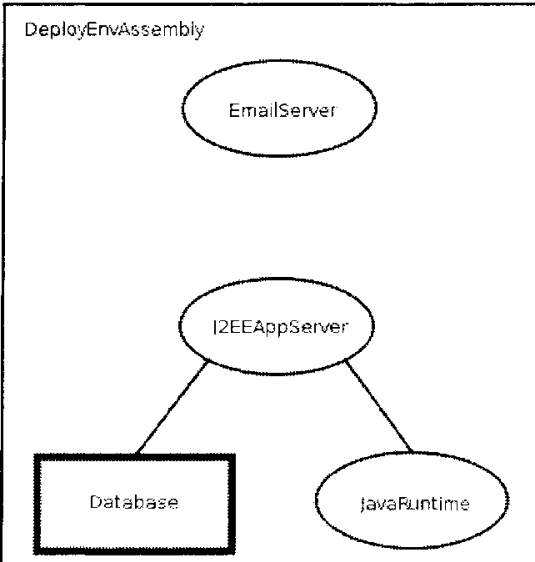

Figure 6.13: The DeployEnvAssembly assembly of the Witan Web system.

\section{Components}

The DeployEnvAssembly assembly contains the following components (Figure 6.13):

- J2EEAppServer: This represents the J2EE compliant application server. It serves as the container in which the Witan Web product is deployed. This is 
defined as a virtual component because there exists several application servers that may be used to deploy the Witan Web product.

- JavaRuntime: This represents the Java runtime environment. It is required by the application server and Witan Web product in order to function. This is defined as a virtual component because there are several JREs available that may be used to execute the Java byte code of the application server and Witan Web product.

- Database: This represents the database that is used for storing the data within the Witan Web product. It is a composite component as it is decomposed into a Java Database Connectivity (JDBC) driver and a database server (see Section 6.3.14).

- EmailServer: This represents the outgoing email server. It is used for sending email notifications to registered users within a conference. This is defined as a virtual component because each organization deploying the Witan Web product may use a different outgoing email server for sending email messages.

\section{Dependencies}

The dependencies defined in DeployEnvAssembly include:

- (J2EEAppServer, JavaRuntime): This represents the dependency relationship between the J2EE application server and the Java runtime environment. This dependency exists as a result of the application server requiring a Java runtime executing compiled Java bytecode.

- (J2EEAppServer, Database): This represents the dependency relationship between the J2EE application server and the database. This dependency exists as a result of the application server requiring a JDBC driver for accessing the database server in order to persist the data of the Witan Web product. 


\section{Constraints}

The (J2EEAppServer, JavaRuntime) depondency has a single constraint associated with it that checks to ensure that the Java runtime environment supports the version of the Java programming language required by the J2EE application server. This constraint is shown in Table 6.12.

\begin{tabular}{|l|l|}
\hline Identifier & JreVersion \\
\hline Description & $\begin{array}{l}\text { The J2EE application server requires that the Java runtime } \\
\text { environment support the proper version of the Java programming } \\
\text { language. }\end{array}$ \\
\hline
\end{tabular}

Table 6.12: The JreVersion constraint that is associated with the (J2EEAppServer, JavaRuntime) dependency.

The constraints associated with the (J2EEAppServer, Database) dependency include the restrictions that exist in configuring the application server to use the database. This includes ensuring that a valid account is provided for accessing the database, as well as requiring that the JDBC driver exists in a location such that it may be loaded by the application server. A sample of the constraints associated with this dependency is shown in Table 6.13.

\begin{tabular}{|l|l|}
\hline Identifier & ValidDbAccount \\
\hline Description & $\begin{array}{l}\text { The J2EE application server requires that a valid account be } \\
\text { provided for accessing the database. The account must have read } \\
\text { and write privileges. }\end{array}$ \\
\hline \hline Identifier & JdbcDriverInClasspath \\
\hline Description & $\begin{array}{l}\text { The J2EE application server requires that the JDBC driver runtime } \\
\text { be located in the Classpath in order for it to be loaded properly. }\end{array}$ \\
\hline
\end{tabular}

Table 6.13: A sample of the constraints associated with the (J2EEAppServer, Database) dependency. 


\subsubsection{DatabaseAssembly}

The DatabaseAssembly assembly represents the database that is used to persist data within the Witan Web system. This consists of both the database server and the JDBC driver that is required in order to communicate with the database server using the Java programming language. The DatabaseAssembly assembly is shown in Figure 6.14 .

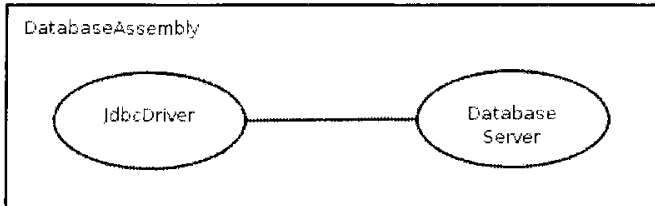

Figure 6.14: The DatabaseAssembly assembly.

\section{Components}

The DatabaseAssembly assembly contains two virtual components (Figure 6.14):

- DatabaseServer

- JdbcDriver

The DatabaseServer component represents the database server that is responsible for storing data within the Witan Web system. This is defined as a virtual component because there exists several database servers that may be used within the Witan Web system. Once the decision has been made as to which server will be used, the DatabaseServer virtual component may be replaced with the appropriate concrete or composite component.

The JdbcDriver component represents the JDBC driver that is required to allow the J2EE application server to communicate with the database server. This is defined as a virtual component because the selection of the JDBC driver to be used is dependent upon the database server selected. Once the database server has been selected, it is possible to select the appropriate JDBC driver to be used within the 
Witan Web system. Then, the JdbcDriver virtual component may be replaced with the appropriate concrete or composite component.

\section{Dependencies}

The only dependency defined in DatabaseAssembly is:

- (JdbcDriver, DatabaseServer)

This dependency exists as a result of the JDBC driver needing to be able to connect to the database server.

\section{Constraints}

The (JdbcDriver, DatabaseServer) dependency has a single constraint associated with it that requires the JDBC driver to be the proper version in order to communicate with the database server. This constraint is shown in Table 6.14.

\begin{tabular}{|l|l|}
\hline Identifier & JdbcDriverVersion \\
\hline Description & $\begin{array}{l}\text { The JDBC driver must be the proper version in order to } \\
\text { communicate with the database server. }\end{array}$ \\
\hline
\end{tabular}

Table 6.14: The JdbcDriverVersion constraint that is associated with the (JdbcDriver, DatabaseServer) dependency.

\subsection{Selecting a View for the Witan Web Product}

This section describes how different variations of the Witan Web product mav be assembled by selecting different views. As mentioned in Section 6.3.11, there are two implementations of the view portion of the Witan Web product:

- A JavaServer Pages (JSP) [17] based view. JSP is the standard tool for creating the presentation layer of a $\mathrm{J} 2 \mathrm{EE}$ application. 
- A Velocity [43] based view. Velocity is a Java based template engine developed by the Apache Jakarta Project [39] that may be used to create the web presentation layer of J2EE applications, and it provides a light-wcight alternative to JSP.

Both of these implementations provide the same functionality; however, they have been developed using different technologies. By selecting either of the available views, it is possible to produce a different instance of the Witan Web product.

To reflect the selection of either view, a specialization must be defined. Each specialization is defined by replacing the View virtual component, which is contained within the WebModuleAssembly (Figure 6.11), with the appropriate concrete or composite component.

If the JSP view is selected, then the View virtual component is replaced with the Jsp View composite component (see Section 6.4.1). The Jsp View composite component is decomposed into a set of components that include the JSP pages, which implement the view, as well as the JSP tag libraries that are used within the view.

If the Velocity view is selected, then the View virtual component is replaced with the VelocityView composite component (see Section 6.4.2). The VelocityView composite component is decomposed into a set of components that include the Velocity pages, which implement the view. Also included is the library containing the Velocity template engine and the classes required to integrate Velocity with the Struts framework, as well as the configuration files required to use Velocity within the Witan Web product.

Depending upon the view selected, the constraints associated with (View, WebConfig) dependency that arise as a result of each selection differ. For example, selecting the JSP view results in a set of constraints related to configuring the JSP tag libraries within the web.xml deployment descriptor. Selecting the Velocity view results in a set of constraints related to configuring the Velocity engine within the web.xml deployment descriptor.

This section defines each of the specializations that reflect the selection of the JSP view and the Velocity view. A sample of the constraints identified within each specialization are also presented. 


\subsubsection{JSP View}

The JSP based view uses JavaServer Pages for the web view in order to display dynamic content to the user. The Hypertext Markup Language (HTML) is used for presentation. and programming logic is embedded within the JSP pages using either the Java programming language or JSP tag libraries.

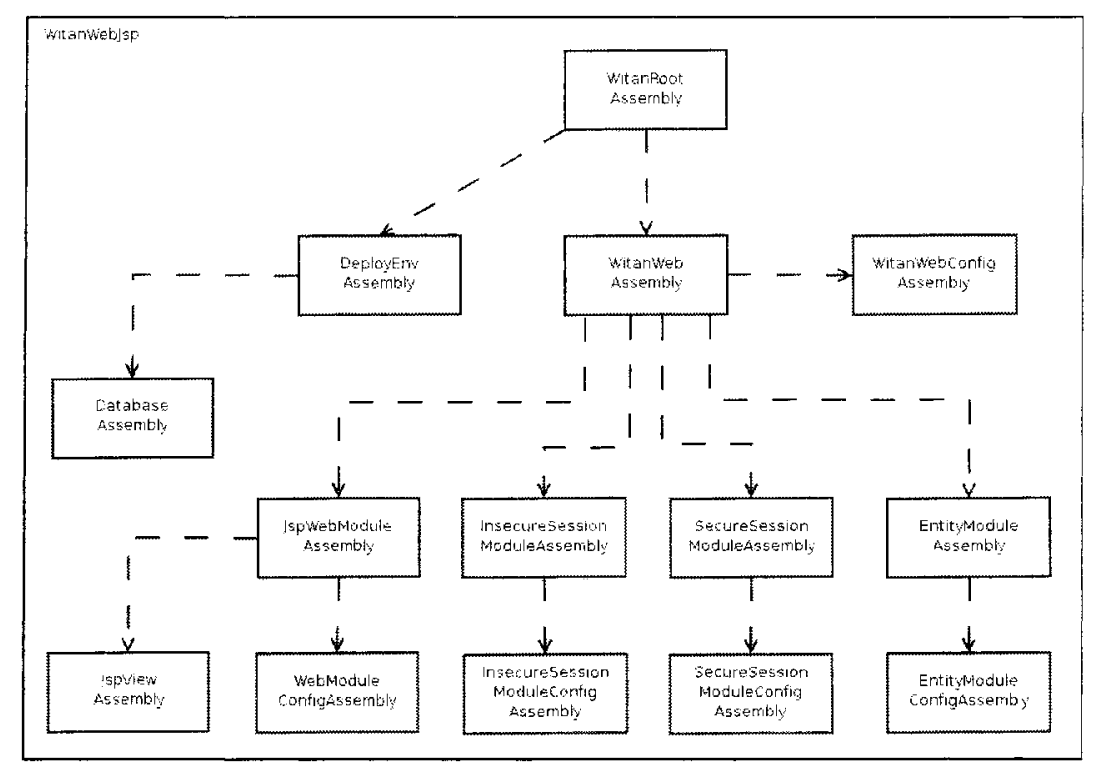

Figure 6.15: The WitanWebJsp configuration, which uses a JSP-based view in the web front-end. This is a specialization of the WitanWebBase configuration (Figure $6.1)$.

Figure 6.15 shows the Witan WebJsp configuration, which is a specialization of the WitanWebBase configuration (see Section 6.3). This specialization is created by replacing the virtual component, View, contained in the WebModuleAssembly assembly (see Section 6.3.11), with the composite component JspView in the JspWebModuleAssembly assembly (Figure 6.16).

The decomposition assembly, Jsp ViewAssembly, of the Jsp View composite component consists of the JSP pages, which are responsible for the presentation of the content in the web view. It also contains a set of JSP tag libraries, which are used for providing common functionality across all of the JSP pages without having to embed 


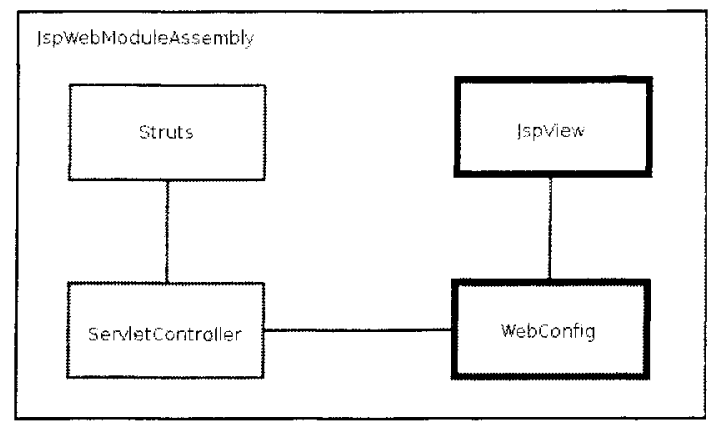

Figure 6.16: The JspWebModuleAssembly assembly. The Jsp View component in this assembly replaces the View virtual component in WebModuleAssembly (Figure 6.11).

large amounts of Java code within the JSP pages. The Jsp ViewAssembly assembly is shown in Figure 6.17

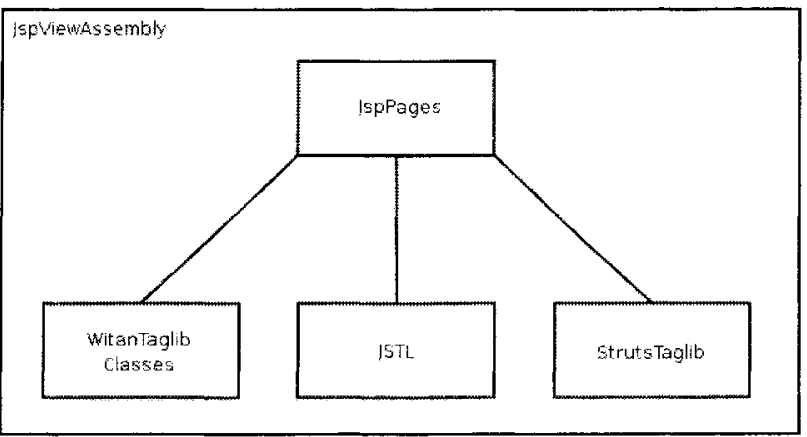

Figure 6.17: The Jsp ViewAssembly assembly. This is the decomposition assembly of the Jsp View composite component in JspWebModuleAssembly (Figure 6.16).

\section{Components}

The JspViewAssembly assembly contains the following components (Figure 6.17):

- WitanTaglib: This represents the Witan tag library. The Witan tag library provides common functionality for retrieving conference properties within the Witan Wob product. 
- StrutsTaglib: This represents the Struts tag library. The Struts tag library provides a set of tags for easing the development of JSP pages in a web application that uses the Struts framowork.

- JSTL: This represents the JavaServer Pages Standard Tag Library (JSTL). The JSTL provides a set of standard tags that perform functionality commonly required within web applications developed using JSP.

- JspPages: This represents the set of JSP pages that make up the view of the web module.

\section{Dependencies}

The dependencies defined in Jsp ViewAssembly include:

- (JspPages, WitanTaglib): This represents the dependency between the JSP pages and the Witan tag library. This dependency exists as the JSP pages require the tags defined within the Witan tag library.

- (JspPages, StrutsTaglib): This represents the dependency between the JSP pages and the Struts tag library. This dependency exists as the JSP pages require some of the tags defined within the Struts tag library.

- (JspPages, JSTL): This represents the dependency between the JSP pages and the JavaServer Pages Standard Tag Library (JSTL). This dependency exists as the JSP pages require some of the tags defined within the JSTL.

\section{Constraints}

The constraints associated with the (JspPages, Witan Taglib), (JspPages, StrutsTaglib) and (JspPages, JSTL) dependencies include ensuring that the proper versions of the tag libraries are used and that the tag libraries are located in a directory that is included on the class path of the web module.

A sample of the constraints associated with these dependencies are shown in Table 6.15 . 


\begin{tabular}{|l|l|}
\hline Identifier & Struts TaglibVersion \\
\hline Description & $\begin{array}{l}\text { The JSP pages require that the Struts tag library be version } 1.1 \\
\text { or greater. }\end{array}$ \\
\hline Rule Set & 1. Check that the version of the Struts framework is 1.1 or greater. \\
\hline \hline Identifier & JSTLJarLocation \\
\hline Description & $\begin{array}{l}\text { The JSTL Jar file must be located in a directory that is available } \\
\text { to the class loader of the web module. }\end{array}$ \\
\hline Rule Set & $\begin{array}{l}\text { 1. Check that JSTL Jar file is located in the WEB-INF/lib directory } \\
\text { of the web application archive (.war file). }\end{array}$ \\
\hline
\end{tabular}

Table 6.15: A sample of the constraints that are associated with the (JspPages, WitanTaglib), (JspPages, StrutsTaglib) and (JspPages, JSTL) dependencies.

As well, by selecting the JSP view, it is possible to define the constraints associated with the (View, WebConfig) dependency that had previously been declared (see Section 6.3.11). An example of a constraint that has been defined once the JSP view was selected is shown in Table 6.16.

\subsubsection{Velocity View}

The Velocity based view uses the Velocity template engine and a set of Velocity pages to provide the presentation layer of the web module. The Velocity pages use HTML for the graphical presentation, and Velocity directives are used for providing dynamic content.

Figure 6.18 shows the Witan Web Velocity configuration, which is a specialization of the Witan WebBase configuration (see Section 6.3). This specialization is created by replacing the View virtual component, which is located in the WebModuleAssembly assembly (see Section 6.3.11). with the VelocityView composite component in the VelocityWebModuleAssembly (Figure 6.19).

The decomposition assembly, Velocity ViewAssembly, of the VelocityView composite component consists of the Velocity pages, which are responsible for providing the presentation logic of the web view. It also consists of the Velocity tools library, which contains the Velocity template engine and the classes required for using Velocity with 


\begin{tabular}{|l|l|}
\hline Identifier & JspRegistrationAction \\
\hline Description & $\begin{array}{l}\text { The registration action must be configured in the } \\
\text { struts-config.xml configuration file. }\end{array}$ \\
\hline Rule Set & $\begin{array}{l}\text { 1. Open the struts-config.xml configuration file. } \\
\text { 2. Check for the action element with the path attribute set to } \\
\text { /registration. } \\
\text { 3. Ensure that the type attribute is set to } \\
\text { nrc.witan.controller.action.conferencestatus. RetrieveConferenceStatus } \\
\text { 4. Ensure that the input attribute is set to /login.jsp. } \\
\text { 5. Ensure that the name attribute is set to ButtonForm. } \\
\text { 6. Ensure that the scope attribute is set to request. } \\
\text { 7. Ensure that validate attribute is set to false. } \\
\text { 8. Ensure that there is a set-property element with the property } \\
\text { attribute set to securityPolicy and the value attribute set to None. } \\
\text { 9. Ensure that there is a forward element with the name attribute set } \\
\text { to success and the path attribute set to /registration.jsp. }\end{array}$ \\
\hline
\end{tabular}

Table 6.16: A sample of the constraints associated with the (Jsp View, WebConfig) dependency. 


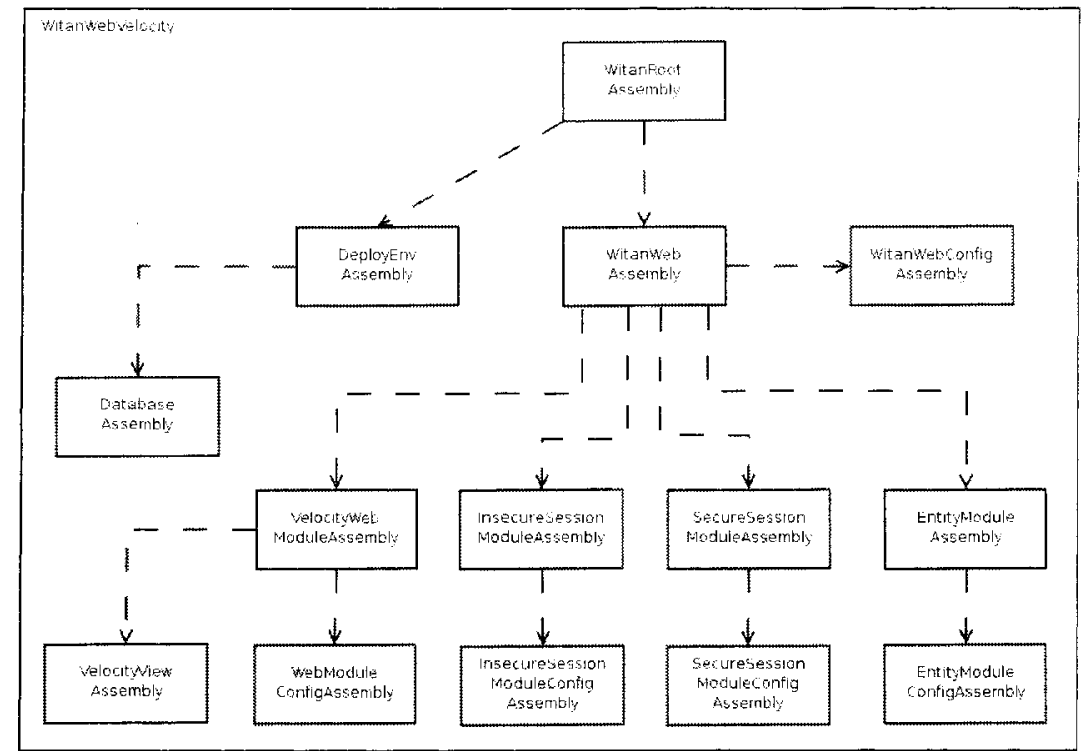

Figure 6.18: The Witan Web Velocity configuration, which uses a Velocity-based view in the web front-end. This is a specialization of the Witan WebBase configuration (Figure 6.1).

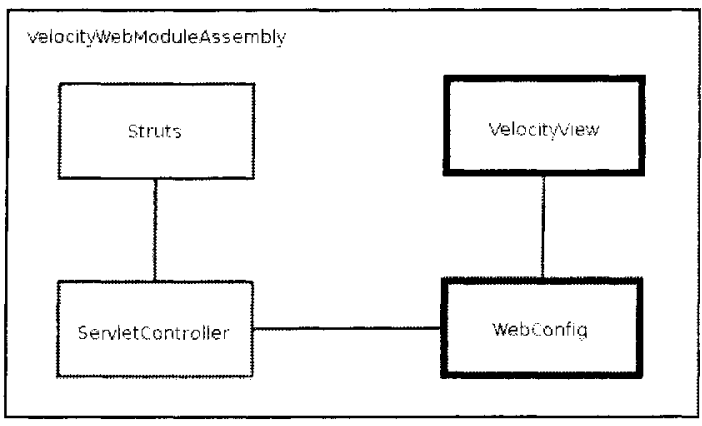

Figure 6.19: The VelocityWebModuleAssembly assembly. The VelocityView component in this assembly replaces the View virtual component in WebModuleAssembly (Figure 6.11). 
the Struts framework. Finally, it contains the configuration files that contain the properties that must be set in order to use Velocity within the Witan Web product. The VelocityViewAssembly assembly is shown in Figure 6.20.

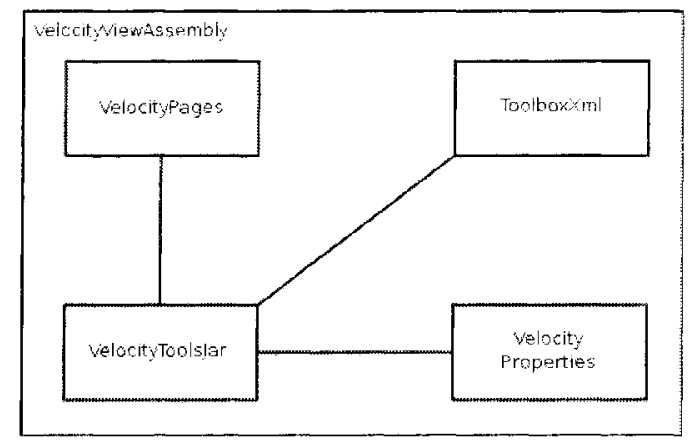

Figure 6.20: The VelocityViewAssembly assembly. This is the decomposition assembly of the VelocityView composite component, which is located in the VelocityWebModuleAssembly assembly (Figure 6.19).

\section{Components}

The Velocity ViewAssembly consists of the following components:

- VelocityPages: This represents the set of Velocity pages that provide the presentation laver of the web module.

- VelocityToolsJar: This represents the library containing the Velocity template engine and the classes required to use Velocity with the Struts framework.

- VelocityProperties: This represents the velocity.properties configuration file that is used to configure the Velocity template engine.

- ToolboxXml: This represents the toolbox.xml configuration file that is used to configure the classes used to integrate Velocity with the Struts framework. 


\section{Dependencies}

The VelocityViewAssembly assembly includes the (VelocityPages, VelocityToolsJar) dependency, which represents the dependency between the VelocityPages and Velocity ToolsJar components. This dependency exists as the Velocity pages require the Velocity template engine, as well as the classes that integrate the template engine and the Struts framework.

Also included within the VelocityViewAssembly assembly are two dependencies between the Velocity template engine and the configuration files. These include:

- (VelocityToolsJar, VelocityProperties)

- (VelocityToolsJar, ToolboxXml)

Each of these dependencies exist as the Velocity template engine must be properly configured within the Witan Web product in order to parse and compile the Velocity pages, as well as integrate with the Struts framework.

\section{Constraints}

The cosntraints associated with the (VelocityPages, VelocityToolsJar) dependency are related to the version of the Velocity tools library that is required to render the Velocity pages. A sample of these constraints is shown in Table 6.17.

\begin{tabular}{|l|l|}
\hline Identifier & Velocity ToolsJarVersion \\
\hline Description & $\begin{array}{l}\text { The version of the Velocity tools library must be } 1.1 \text { or } \\
\text { greater. }\end{array}$ \\
\hline Rule Set & $\begin{array}{l}\text { 1. Open the WEB-INF/lib directory of the web module. } \\
\text { 2. Check that the velocity-tools-1.x.jar file exists, where } \\
\mathrm{x} \text { is greater than or equal to } 1 .\end{array}$ \\
\hline
\end{tabular}

Table 6.17: The VelocityToolsJarVersion constraint that is associated with the (VelocityPages, Velocity ToolsJar) dependency.

The constraints associated with the (VelocityToolsJar. VelocityProperties) and 
(VelocityToolsJar, ToolboxXml) dependencies are related to the configuration properties that must be set in order for the Velocity template engine to properly parse and compile Velocity pages, as well as integrate with the Struts framework. A sample of these constraints is shown in Table 6.18.

\begin{tabular}{|l|l|}
\hline Identifier & TilesSupportDefinition \\
\hline Description & $\begin{array}{l}\text { The toolbox.xml configuration file must define a tool } \\
\text { to support usage of Tiles within the Velocity pages. }\end{array}$ \\
\hline Rule Set & $\begin{array}{l}\text { 1. Open the toolbox.xml configuration file, which is } \\
\text { located within the WEB-INF/ directory of the web module. } \\
\text { 2. Check for the definition of a tool with the key element } \\
\text { set to tiles. } \\
\text { 3. Ensure that the value of the scope element is set to request. } \\
\text { 4. Ensure that the value of the class element is set to: } \\
\text { org.apache.velocity.tools.struts. TilesTool }\end{array}$ \\
\hline
\end{tabular}

Table 6.18: A sample of the constraints associated with the (VelocityToolsJar, VelocityProperties) and (VelocityToolsJar, Toolbox Xml) dependencies.

As well, by selecting the Velocity view, it is possible to define the constraints associated with the (View, WebConfig) dependency that had previously been declared (see Section 6.3.11). An example of a constraint that has been defined once the Velocity view was selected is shown in Table 6.19 .

\subsubsection{Results for Selecting Different Views}

Selecting different views to be included within the Witan Web software product resulted in different sets of constraints for each view.

The JSP and Velocity views are each composed of a set of components and dependencies that are different in both instances. The JSP view consists of the set of JSP pages and the tag libraries that are used within the JSP pages. The constraints that are associated with the dependencies between these components include the restrictions on using the proper versions of the included JSP tag libraries.

The Velocity view consists of the set of Velocity pages and the Velocity template 


\begin{tabular}{|l|l|}
\hline Identifier & VelocityRegistrationAction \\
\hline Description & $\begin{array}{l}\text { The Registration action must be configured in the } \\
\text { struts-config.xml configuration file. }\end{array}$ \\
\hline Rule Set & $\begin{array}{l}\text { 1. Open the struts-config.xml configuration file. } \\
\text { 2. Check for the action element with the path attribute set to } \\
\text { /registration. } \\
\text { 3. Ensure that the type attribute is set to } \\
\text { nrc.witan.controller.action.conferencestatus. RetrieveConferenceStatus } \\
\text { 4. Ensure that the input attribute is set to /login.vm. } \\
\text { 5. Ensure that the name attribute is set to ButtonForm. } \\
\text { 6. Ensure that the scope attribute is set to request. } \\
\text { 7. Ensure that validate attribute is set to false. } \\
\text { 8. Ensure that there is a set-property element with the property } \\
\text { attribute set to securityPolicy and the value attribute set to None. } \\
\text { 9. Ensure that there is a forward element with the name attribute set } \\
\text { to success and the path attribute set to / registration.vm. }\end{array}$ \\
\hline
\end{tabular}

Table 6.19: A sample of the constraints associated with the (VelocityView, WebConfig) dependency. 
engine, as well as configuration files required by the Velocity template enginc. The constraints associated with the dependencies between these products include the restrictions on using the proper version of the Velocity engine, as well as the restrictions on the configuration of the Velocity engine in order to allow it to be integrated with the Struts framework.

Depending upon whether the JSP or Volocity view was selected, the constraints associated with the (JspView, WebConfig) dependency or the (VelocityView, WebConfig) dependency, respectively, define the restrictions placed upon the Witan Web product in order to ensure that the selected view was properly configured.

When the ISP view was selected, a set of constraints associated with the (Jsp View, WebConfig) dependency were identified that pertain to the configuration of the JSP tag libraries that are used within the view.

However, when the Velocity view was selected, a set of constraints associated with the (VelocityView, WebConfig) dependency were discovered that were related to the configuration of the Velocity template engine, which is implemented as a Java Servlet.

Finally, all of the constraints associated with the (View, WebConfig) dependency, which had previously been declared, were defined for each view. These constraints pertain to the actions that must be configured within the struts-config.xml file, and the definition of these constraints specify the appropriate pages in the JSP or Velocity view that are related to each action.

\subsection{Deploying the Witan Web System on Different Application Servers}

The Witan Web software product requires a J2EE application server in order to be deployed. There are numerous J2EE application servers available that may fill this requirement, including:

- JBoss

- BEA WebLogic 
- IBM WebSphere

- Orion

Each organization that is deploving the Witan Web software product may select a different application server. The decision to use a particular application server may be based upon requirements, pricing, and/or availability.

Selecting a particular application server requires that a specialization be defined to reflect the decision. The specialization is created by replacing the J2EEAppServer virtual component, which is located in the DeployEnvAssembly (Figure 6.13), with the concrete or composite component that represents the application server selected.

Each application server that is selected results in a unique set of constraints that must be specified in the appropriate configuration.

Any previously declared constraints, which are associated with the virtual component representing the $\mathrm{J} 2 \mathrm{EE}$ application server, may be defined once an application server has been selected.

To demonstrate how the selection of different application servers effects the constraints that are specified within a system, this section presents the specializations that have been defined when selecting the JBoss v3 (see Section 6.5.1) and JBoss v4 (see Section 6.5.2) application servers.

Selecting each of these application servers results in a different set of constraints that must be specified in the appropriate specialization.

Selecting the JBoss v3 and JBoss v4 application servers require that the JBoss specific deployment descriptors be included within the Witan Web configuration, as well as each of the modules contained within the Witan Web product (sec Section 6.5.3). This results in a set of constraints that are common to both application servers.

Each of the specializations that reflect the selection of each of these application servers are described in this section. Also, a sample of the constraints that ariso from selecting these components is presented. 


\subsubsection{JBoss 3}

Selecting JBoss v3 as the application server on which the Witan Web software product is deployed results in the WitanWebJBoss3 configuration (Figure 6.21). WitanWebJBoss? is a specialization of the WitanWebBase configuration (see Soction 6.3).

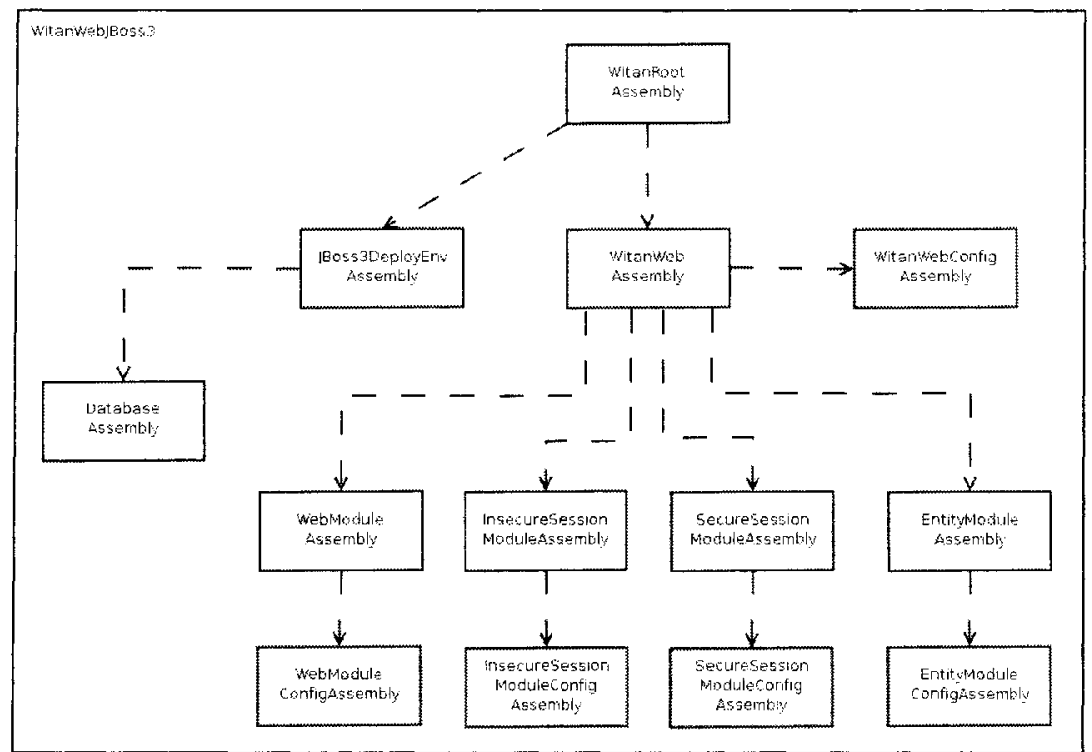

Figure 6.21: The WitanWebJBoss3 configuration, which uses JBoss v3 as the J2EE application server. This is a specialization of the WitanWebBase configuration (see Figure 6.1).

This specialization is created by replacing the J2EEAppServer virtual component, contained in the DeployEnvAssembly assembly (see Section 6.3.13), with the JBoss3 concrete component in the JBoss3DeployEnvAssembly (Figure 6.22).

Deploying the Witan Web software product on the JBoss v3 application server results in several new constraints being identified, which are associated with the (WitanWeb, DeployEnv) dependency contained in the WitanRootAssembly assembly (see Section 6.3.2). A sample of these newly discovered constraints is shown in Table 6.20 . 


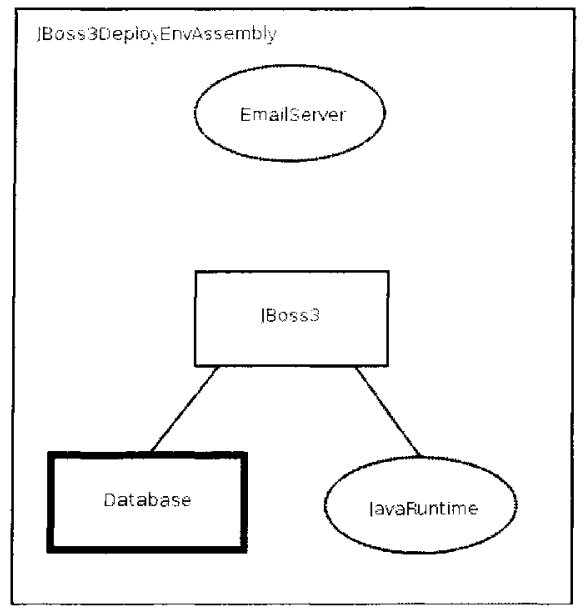

Figure 6.22: The JBoss3DeployEnvAssembly assembly. The JBoss3 concrete component in this assembly replaces the J2EEAppServer virtual component in DeployEnvAssembly (see Figure 6.13).

\begin{tabular}{|l|l|}
\hline Identifier & JBoss3LoginModule \\
\hline Description & $\begin{array}{l}\text { The database server login module must be configured in the } \\
\text { JBoss v3 application server. }\end{array}$ \\
\hline \hline Identifier & JBoss3JavaHeapSpace \\
\hline Description & $\begin{array}{l}\text { The Java heap space needs to be set to a large number in order to } \\
\text { accomodate memory intensive processes such as the report generation } \\
\text { facility. }\end{array}$ \\
\hline Rule Set & $\begin{array}{l}\text { 1. Go to the <jboss3_home>/bin directory, where <jboss3_home> } \\
\text { is the installation directory of the JBoss v3 application server. } \\
\text { 2. Open the run.bat (for Windows) or run.sh (for Linux) startup } \\
\text { script. } \\
\text { 3. Add the following line to the startup script: } \\
\text { JAVA_OPTS = \%JAVA_OPTS\% -Xms128m Ximx1024m } \\
\text { (use \$JIAVA_OPTS in place of \%JAVA_OPTS\% on Linux) }\end{array}$ \\
\hline
\end{tabular}

Table 6.20: A sample of constraints that have been identified as a result of selecting JBoss v3 as the J2EE application server within the Witan Web system. 
As well, constraints which had previously been declared may now also be defined by specifying the rule set for checking the constraint. A sample of previously declared constraints that have been defined following the selection of the JBoss v3 application server is shown in Table 6.21.

\begin{tabular}{|l|l|}
\hline Identifier & Ejb20Support \\
\hline Description & $\begin{array}{l}\text { The Witan Web product uses Container Managed Persistence } \\
\text { (CMP) relationships which were first defined in the }\end{array}$ \\
& $\begin{array}{l}\text { Enterprise JavaBean (EJB) } 2.0 \text { specification. Therefore, } \\
\text { the J2EE application server must provide an EJB } \\
\text { container that implements at least the EJB 2.0 } \\
\text { specification. }\end{array}$ \\
\hline Rule Set & 1. JBoss 3.x implements the EJB 2.0 specification. \\
\hline
\end{tabular}

Table 6.21: A sample of previously declared constraints that have been defined once JBoss $\mathrm{v} 3$ has been selected as the J2EE application server.

\subsubsection{JBoss 4}

Selecting JBoss v3 as the J2EE application server results in the Witan WebJBoss 4 configuration (Figure 6.23), which is a specialization of the WitanWebBase configuration (see Section 6.3).

This specialization is created by replacing the J2EEAppServer virtual component, contained in the DeployEnv assembly (see Section 6.3.13), with the JBoss 4 concrete component in the JBoss4DeployEnvAssembly assembly. The JBoss4DeployEnvAssembly assembly is shown in Figure 6.24.

Deploying the Witan Web software product on the JBoss v4 application server results in several newly indentifed constraints, which are associated with the ( $W i$ tanWeb, DeployEnv) dependency in the WitanRootAssembly assembly (see Section 6.3.2). A sample of the newly discovered constraints is shown in Table 6.22.

As well, constraints which had previously been declared may now also be defined by specifying the rule set for checking the constraint. A sample of previously declared 


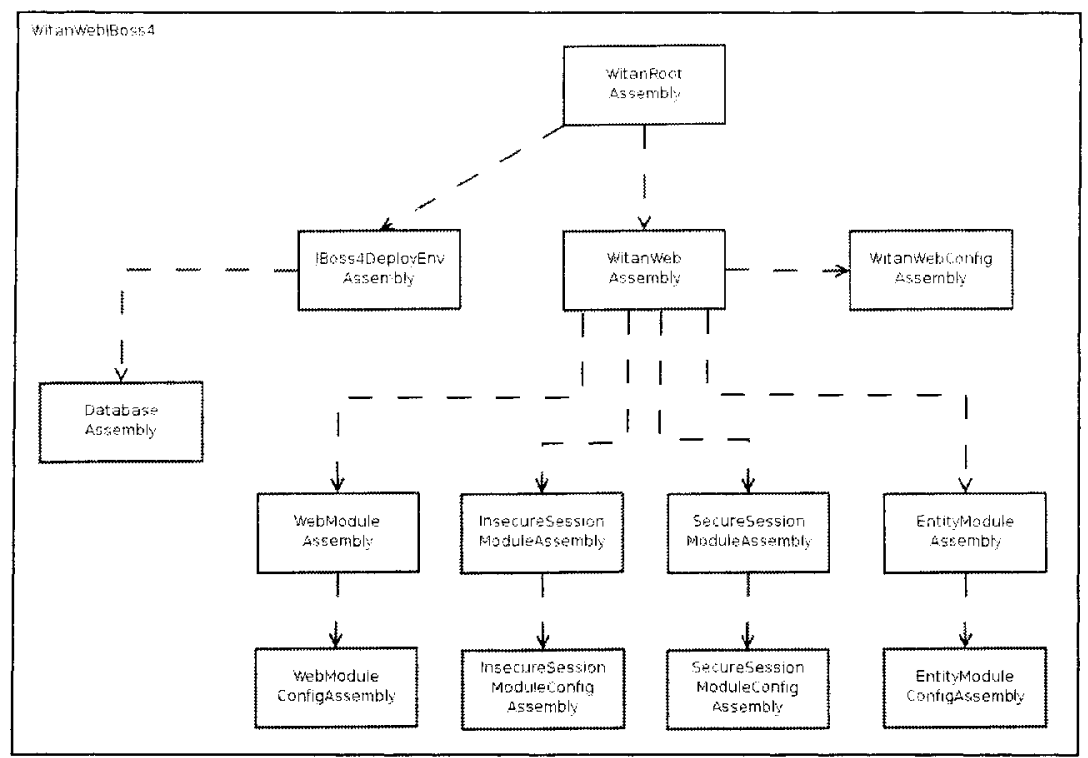

Figure 6.23: The WitanWebJBoss4 configuration, which uses JBoss v4 as the J2EE application server. This is a specialization of the WitanWebBase configuration (see Figure 6.1).

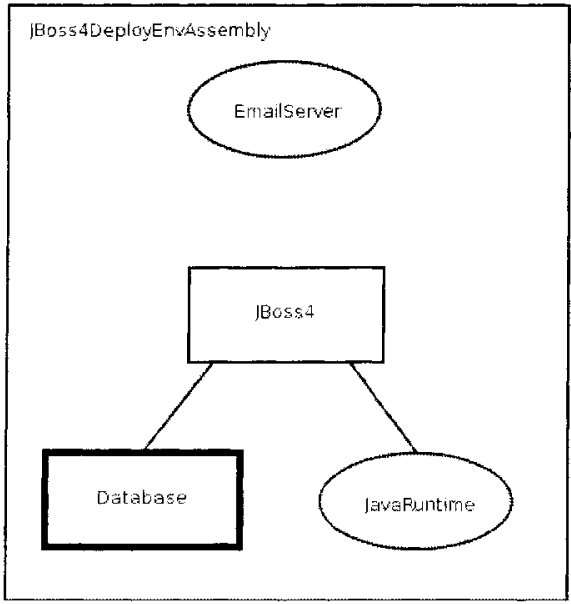

Figure 6.24: The JBoss4DeployEnvAssembly assembly. The JBoss4 concrete componont in this assembly replaces the J2EEAppServer virtual component in DeployEn$v$ Assembly (see Figure 6.13). 


\begin{tabular}{|c|c|}
\hline Identifier & JBoss 4LoginModule \\
\hline Description & $\begin{array}{l}\text { The database server login module must be configured in the } \\
\text { JBoss v4 application server. }\end{array}$ \\
\hline Identifier & JBoss4JavaHeapSpace \\
\hline Description & $\begin{array}{l}\text { The Java heap space needs to be set to a large number in order to } \\
\text { accomodate memory intensive processes such as the report generation } \\
\text { facility. }\end{array}$ \\
\hline Rule Set & $\begin{array}{l}\text { 1. Go to the <jboss4_home }>\text { /bin directory, where <jboss4_home }> \\
\text { is the installation directory of the JBoss } 4 \text { application server. } \\
\text { 2. Open the run.bat (for Windows) or run.sh (for Linux) startup } \\
\text { script. } \\
\text { 3. Add the following line to the startup script: } \\
\text { JAVA_OPTS = \%JAVA_OPTS\% -Xms } 128 \mathrm{~m} \text { Xmx } 1024 \mathrm{~m} \\
\text { (use \$JAVA_OPTS in place of \%JAVA_OPTS\% on Linux) }\end{array}$ \\
\hline Identifier & JBoss4WitanWebVersion \\
\hline Description & $\begin{array}{l}\text { Version } 2.1 \text { or greater of the Witan Web product is required when } \\
\text { using JBoss v4. This is required in order to overcome a change } \\
\text { in JBoss v } 4 \text { where parameters are passes by value instead of by } \\
\text { reference, as they were in JBoss v3. }\end{array}$ \\
\hline Rule Set & $\begin{array}{l}\text { 1. Ensure that version } 2.1 \text { or greater of the Witan Web software } \\
\text { product is being deployed. }\end{array}$ \\
\hline Identifier & UseJBossClassLoader \\
\hline Description & $\begin{array}{l}\text { To deploy the Witan Web software product on JBoss v } 4.0 .2 \text {, the } \\
\text { UseJBossClassLoader property must be set to 'true' for the web } \\
\text { server. }\end{array}$ \\
\hline Rule Set & $\begin{array}{l}\text { 1. Go to the }<\text { jboss } 4 \text { home }>\text { /server/default/conf directory, where } \\
<\text { jboss } 4 \text { home }>\text { is the installation directory of the JBoss v } 4 \\
\text { application server. } \\
\text { 2. Open the jboss-service.xml configuration file. } \\
\text { 3. Locate the mbean element with the name attribute set to } \\
\text { jboss:service=WebService. } \\
\text { 4. Ensure that there exists an attribute element with the name } \\
\text { set to UseJBossClassLoader and the value set to true. }\end{array}$ \\
\hline
\end{tabular}

Table 6.22: A sample of constraints that have been identified as a result of selecting JBoss v4 as the J2EE application server within the Witan Web system. 
constraints that have been defined following the selection of the JBoss $r 4$ application server is shown in Table 6.21 .

\begin{tabular}{|l|l|}
\hline Identifier & Ejb20Support \\
\hline Description & $\begin{array}{l}\text { The Witan Web product uses Container Managed Persistence } \\
\text { (CMP) relationships which were first defined in the } \\
\text { Enterprise JavaBean (EJB) } 2.0 \text { specification. Therefore, } \\
\text { the J2EE application server must provide an EJB } \\
\text { container that implements at least the EJB 2.0 } \\
\text { specification. }\end{array}$ \\
\hline Rule Set & \begin{tabular}{l} 
1. JBoss 4.x implements the EJB 2.x specification. \\
\hline
\end{tabular}
\end{tabular}

Table 6.23: A sample of constraints that were previously declared and have been defined once JBoss $v 4$ has been selected as the J2EE application server within the Witan Web system.

\subsubsection{JBoss Specific Deployment Descriptors}

Regardless of which version of JBoss is selected as the J2EE application server within the Witan Web system, there is a common set of server specific configuration and deployment descriptor files that must be included within the Witan Web software product. These files allow for server specific properties to be set within the product in order for it to take advantage of any non-standard J2EE features offered by the application server.

The configurations WitanWebJBoss3Config and WitanWebJBoss4Config, shown in Figure 6.25 and Figure 6.26, respectively, reflect the inclusion of the JBoss server specific configuration and deployment descriptor files.

Figure 6.27 shows the WitanWebConfigJBossAssembly assembly with the JBossApplicationXmlDD concrete component, which has replaced the ApplicationConfAS virtual component in the WitanWebConfigAssembly assembly (see Section 6.3.4).

Figure 6.28 shows the EntityModuleConfigJBossAssembly assembly with the JBossEntityConfig composite component, which replaces the EntityConfAS virtual component in the EntityModuleConfigAssembly assembly (see Section 6.3.6). 


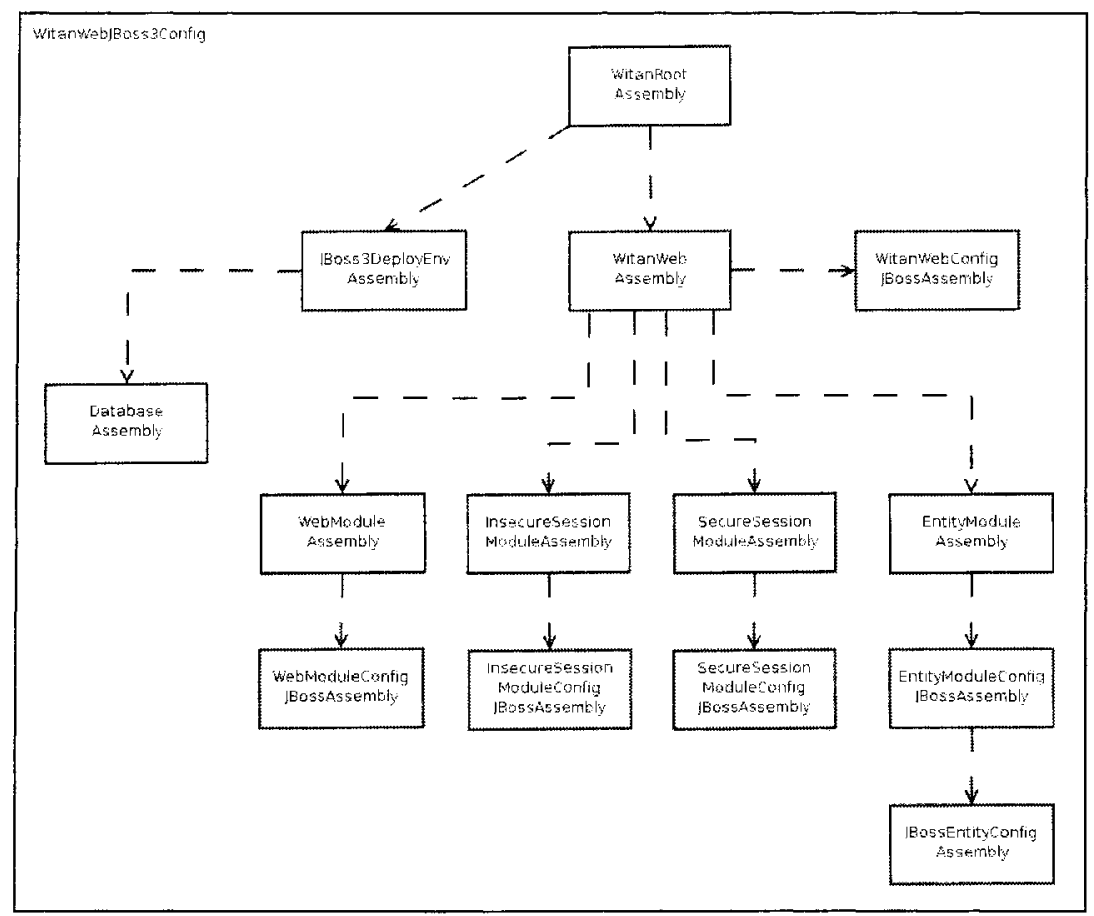

Figure 6.25: The WitanWebJBoss3Config configuration, which uses JBoss v3 as the application server and includes the server specific configuration and deployment descriptor files. 


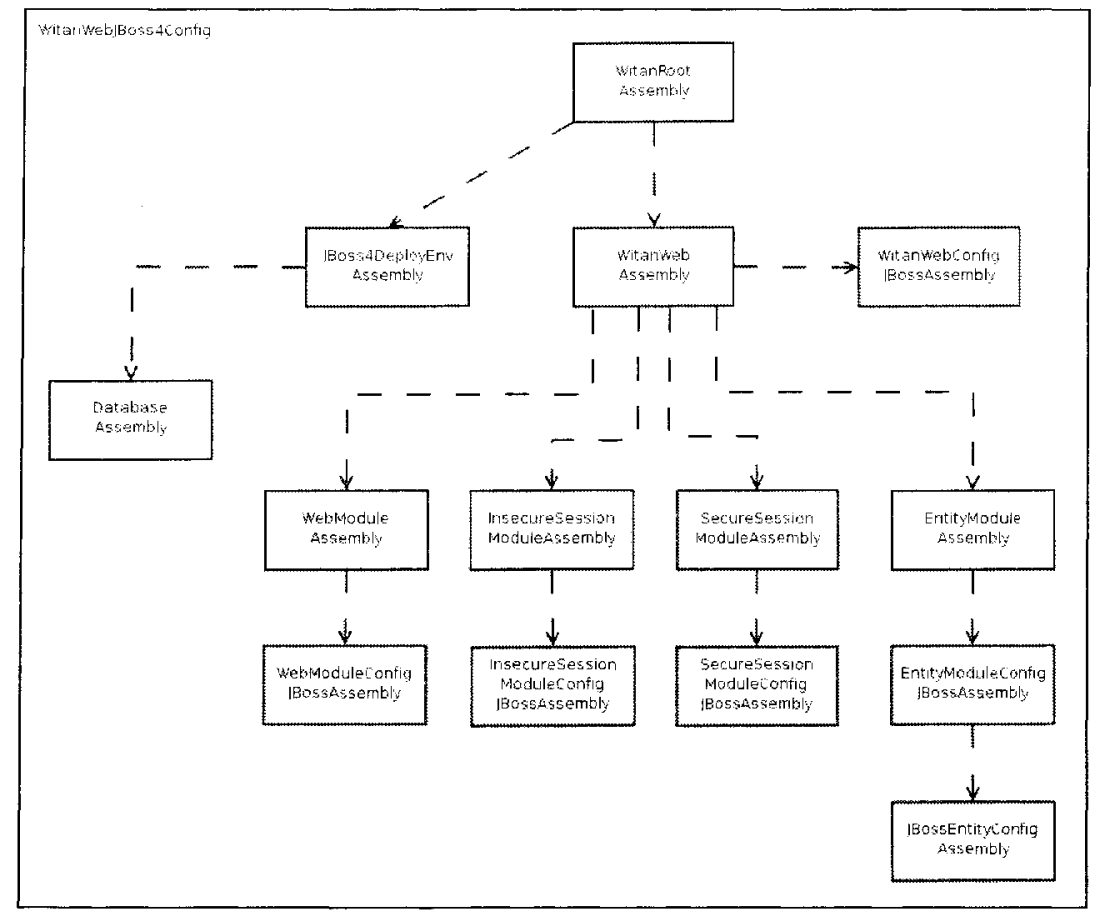

Figure 6.26: The WitanWebJBoss4Config configuration, which uses JBoss v4 as the application server and includes the server specific configuration and deployment descriptor files.

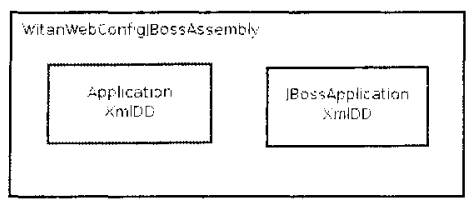

Figure 6.27: The WitanWebConfigJBossAssembly assembly, which includes the JBoss server specific jboss-app.xml deployment descriptor required by the Witan Web software product. 


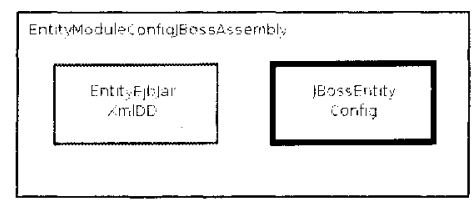

Figure 6.28: The EntityModuleConfigJBossAssembly assembly, which includes the JBoss server specific configuration and deployment descriptor files required within the entity module.

The decomposition assembly of the JBossEntityConfig composite component contains the JBossXmlDD and JBossCmpJbdcXmlDD concrete components (Figure 6.29. The JBossXmlDD component represents the jboss.xml deployment descriptor, which is used to define server specific properties for the entity beans contained in the entity module of the Witan Web product. The JBossCmpJdbcXmlDD component represents the jbosscmp-jdbc.xml deployment descriptor which is used for overriding the default SQL type mappings of the CMP fields of each entity bean. Also, the queries executed by the finder methods contained within the entity beans are specified using the JBoss Query Language.

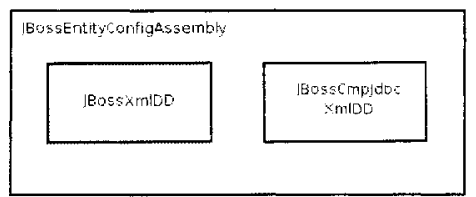

Figure 6.29: The WitanWebConfigJBossAssembly assembly; which includes the JBoss server specific jboss-app.xml deployment descriptor required by the Witan Web software product.

Both the secure session module and the insecure session module do not require any JBoss specific configuration properties to be set; all of the configuration required is contained within the ejb-jar.xml deployment descriptors of the secure and insecure session modules. Thus, the SecureSessionConfAS virtual component, contained in the SecureSessionModuleConfigAssembly assembly (see Section 6.3.8, is replaced with 
the cxisting SecureSessionEjbJarXmlDD concrete component in the SecureSessionModuleConfigJBossAssembly (Figure 6.30). Similarly, the InsecureSessionConfAS virtual component, contained in the InsecureSessionModuleConfigAssembly assembly, is replaced by the existing InsecureSessionEjbJarXmlDD concrete component in the InsecureSessionModule ConfigJBossAssembly assembly (Figure 6.31).

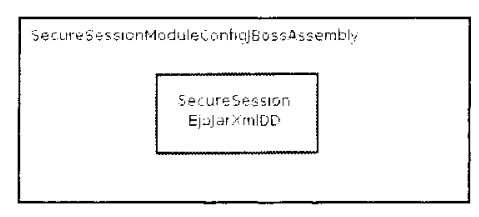

Figure 6.30: The SecureSessionModuleConfigJBossAssembly assembly.

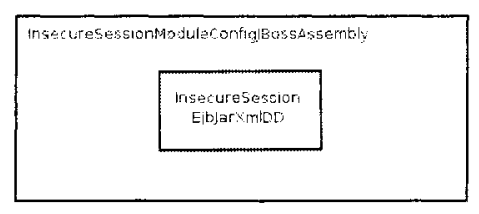

Figure 6.31: The IsecureSessionModuleConfigJBossAssembly assembly.

Figure 6.32 shows the WebModuleConfigJBossAssembly assembly, with the JBossWebXmlDD concrete component, which replaces the WebConfAS virtual component contained in the WebModuleConfigAssembly (see Section 6.3.12). The JBossWe$b X m l D D$ component represents the jboss-web.xml deployment descriptor which is used to define the security realm that is used within the web front-end.

\section{Constraints}

The constraints associated with the dependencies involving the configuration assemblies that contain JBoss server specific deployment descriptors include configuration settings that are required for deploying the Witan Web software product on a JBoss application server. 


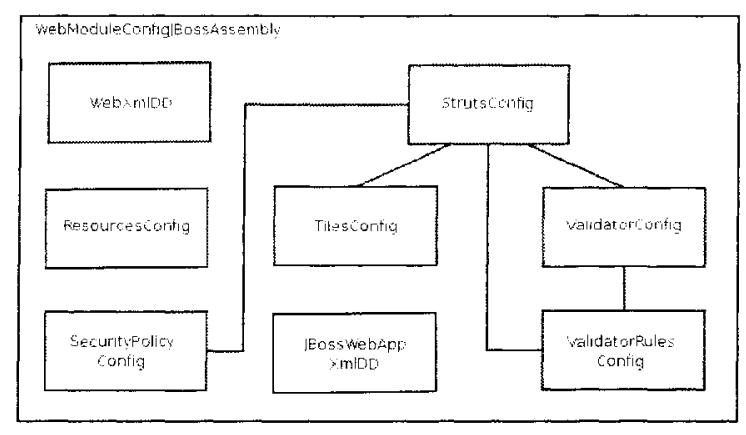

Figure 6.32: The WebModuleConfigJBossAssembly assembly, which includes the JBoss server specific jboss-web.xml deployment descriptor required by the Witan Wob software product.

The majority of these constraints pertain to redefining certain queries using the JBoss Query Language, which overcomes some of the deficiencies in EJB QL. As well, all get methods and value object creation methods within the entity beans must be defined as read only. To demonstrate these restrictions, a sample of the constraints associated with the (PaperEJB, EntityConfig) dependency is shown in Table 6.24.

\subsubsection{Results for Selecting Different Application Servers}

JBoss [18] were careful to provide backward compatibility within new releases of the JBoss application server. As a result, many of the constraints that were identified when the JBoss v3 application server was used were also applicable when the JBoss $\mathrm{v} 4$ application server was selected.

A major benefit of this backward compatibility is that the application server specific configuration files are the same for both versions of JBoss. Thus, a set of specializations were created to represent the inclusion of each JBoss specific configuration file without having to select a particular version of JBoss beforehand. All of the constraints associated with these configuration files could then be specified and included in both specializations created to reflect the selection of the JBoss v3 and JBoss $\mathrm{v} 4$ application server. This prevented from having to redefine all of the constraints for both application servers. 


\begin{tabular}{|c|c|}
\hline Identifier & PaperBeanJBossQLFindAllQuery \\
\hline Description & $\begin{array}{l}\text { There must be a JBoss QL query for the find All method specified } \\
\text { in the definition of the Paper bean in the jbosscmp-jdbc.xml } \\
\text { deployment descriptor of the entity module. }\end{array}$ \\
\hline Rule Set & $\begin{array}{l}\text { 1. Open the jbosscmp-jdbc.xml deployment descriptor of the entity } \\
\text { module. } \\
\text { 2. Locate the definition of the Paper entity bean. } \\
\text { 3. Check that there exists a query element containing a } \\
\text { query-method element with the method-name element set } \\
\text { to findAll. } \\
\text { 4. Check that the jboss-ql element contains an EJB QL query that } \\
\text { retrieves all User beans stored in the system and orders the users } \\
\text { by name. }\end{array}$ \\
\hline Identifier & PaperBeanReadOnlyGetMethods \\
\hline Description & $\begin{array}{l}\text { All of the get methods defined within the the Paper entity bean must } \\
\text { be defined as read only in the jboss.xml deployment } \\
\text { descriptor of the entity module. }\end{array}$ \\
\hline Rule Set & $\begin{array}{l}\text { 1. Open the jboss.xml deployment descriptor of the entity } \\
\text { module. } \\
\text { 2. Located the defintion of the Paper bean. } \\
\text { 3. Check that there exists a method element with the } \\
\text { method-name element set to get*. } \\
\text { 4. Ensure that the read-only element is set to true. }\end{array}$ \\
\hline
\end{tabular}

Table 6.24: A sample of the constraints associated with the (PaperEJB, EntityConfig) dependency, which arise as a result of using the JBoss application server specific configuration files. 
Also, because of the backward compatibility, many of the constraints associated with the (WitanWeb, DeployEnv) dependency were the same for both the JBoss v3 and JBoss v4 application servers. However, the standard configuration of the JBoss $\mathrm{v} 4$ application server was changed from that of JBoss v3, therefore, several new constraints were discovered. These constraints included the need to configure the class loader used within the web server built into JBoss. Also, the Witan Web product had to be updated to accomodate the fact that JBoss $v 4$ passes parameters by value in remote method calls, whereas JBoss v3 passes parameters by reference. Thus, the Witan Web product must be of a proper version in order to be deployed on JBoss $\mathrm{v} 4$.

\subsection{Deploying the Witan Web System with Differ- ent Databases}

The Witan Web product requires a database to persist the conference data. Different organizations may decide to deploy the Witan Web product using different databases, depending upon the criteria for selecting a database. This criteria may include performance requirements, database availability, and pricing.

There are numerous databases available including:

- MySQL

- PostgreSQL

- IBM DB2

- Oracle

- Microsoft SQL Server

Since Witan Web is developed using the J2EE platform, and, thus, the Java programming language, a JDBC driver is required to allow for communication between the application server and the database server. 
Once a database scrver has been selected, a specialization must be defined to reflect the decision. The specialization is created by replacing the DatabaseServer virtual component, which is located in the DatabaseAssembly assembly (Figure 6.14), with the concrete or composite component that represents the database server that has been selected.

Depending upon the database server used, a JDBC driver that is compatible with the database server must be selected. A specialization must then be defined to reflect the decision. The specialization is created by replacing the $J d b c D$ river virtual component, which is located in the DatabaseAssembly assembly (Figure 6.14), with the concrete or composite component that represents the JDBC driver that has been selected.

Each combination of database server and JDBC driver that is selected to be used within the Witan Web system results in a unique set of constraints. These constraints must be identified and specified in the appropriate configuration in order to prevent having to rediscover the constraints each time the same combination of database and JDBC driver are used in deploying the Witan Web product.

Also, any constraints that have previously been declared, and which are associated with the virtual components representing the database server and JDBC driver, may be defined once the database server and JDBC driver have been selected.

To demonstrate how the constraints differ depending upon the database server and JDBC driver selected, this section presents two combinations of products used in this case study:

- The MySQL database server [27] and MySQL Connector/J JDBC driver [26] (see Section 6.6.1)

- The PostgreSQL database server [31] and PostgreSQL JDBC driver [32] (see Section 6.6.2)

Selecting these combinations of database server and JDBC driver result in a set of constraints that must be specified in the appropriate configuration.

This section describes the specializations that were defined to reflect the selection of the database server and JDBC driver combinations mentioned above. A sample of 
the constraints identified when these products are used within the Witan Web system is also presented.

\subsubsection{MySQL}

Selecting the MySQL database server and the MySQL Connector/J JDBC driver to be used within the Witan Web system results in the WitanWebMySql configuration (Figure 6.33). This configuration is derived from the Witan WebBase configuration (see Section 6.3).

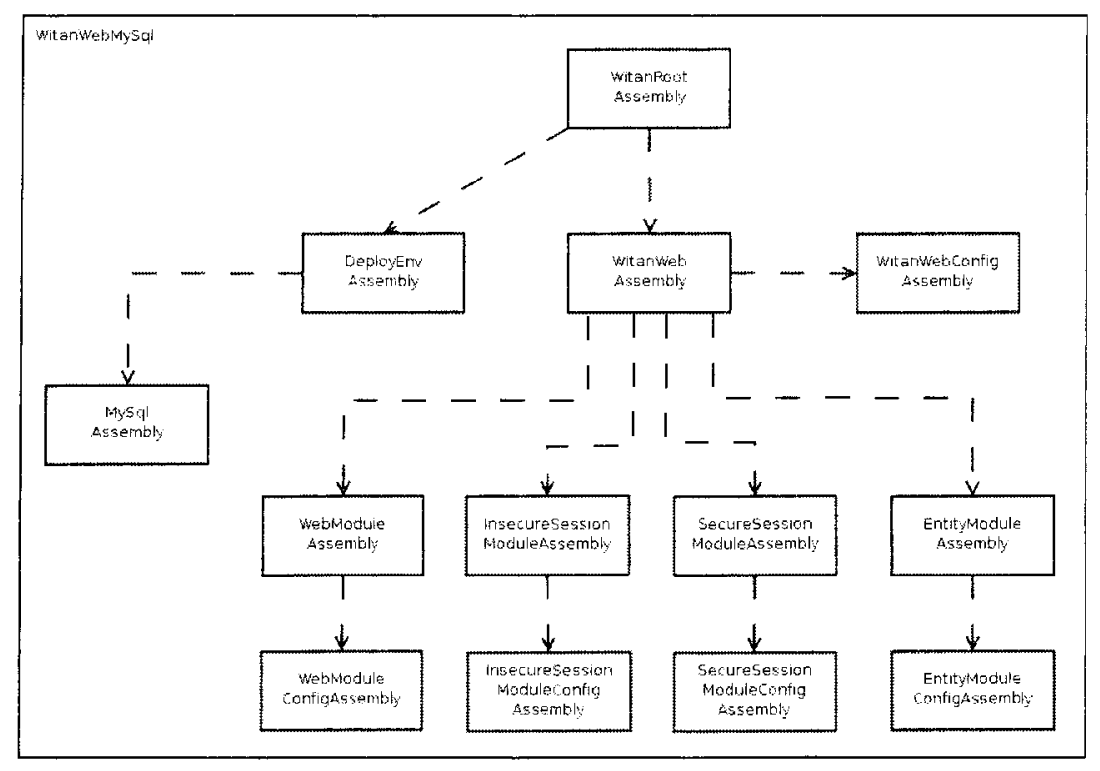

Figure 6.33: The Witan WebMySql configuration, which uses MySQL as the database server and MySQL Connector/J as the JDBC driver.

The MySqlAssembly assembly is created by replacing the DatabaseSever and $J d b c$ Driver virtual components in DatabaseAssembly (see Section 6.3.14) with the MySqlDB and MySqlConnectorJJdbcDriver concrete components (Figure 6.34).

The decision to use these components together within the Witan Web system allows for the JdbcDriverVersion constraint, which had previously been declared (see Section 6.3.14), to be defined. The definition of this constraint is shown in Table 6.25. 


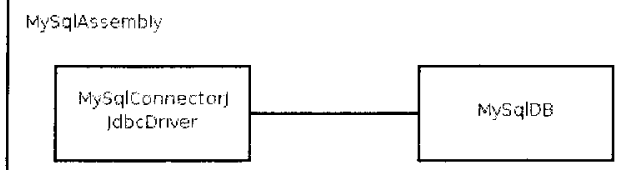

Figure 6.34: The MySqlAssembly assembly. The MySqlConnectorJJdbcDriver and $M y S q L D B$ concrete components in this assembly replace the JdbcDriver and DatabaseServer virtual components, respectively, in DeployEnvAssembly (see Figure 6.14).

\begin{tabular}{|l|l|}
\hline Identifier & JdbcDriverVersion \\
\hline Description & $\begin{array}{l}\text { The JDBC driver must be the proper version in order to } \\
\text { communicate with the database server. }\end{array}$ \\
\hline \hline Rule Set & $\begin{array}{l}\text { 1. The MySQL Connector/J JDBC driver must be version 3.0 or } \\
\text { higher when using version 3.2x or 4.x of the MySQL database } \\
\text { server. }\end{array}$ \\
\hline
\end{tabular}

Table 6.25: The definition of the JdbcDriverVersion constraint with MySql used as the database server and MySQL Connector/J used as the JDBC driver. 
Also, when the MySQL database server is selected to be used in the Witan Web system, it must be configured to allow large packet sizes in order to allow for uploaded paper files to be stored in the database. This restriction is represented by the MySQLMaxPacketAllow constraint, and it is shown in Table 6.26.

\begin{tabular}{|l|l|}
\hline Identifier & MySQLMaxPacketAllow \\
\hline Description & $\begin{array}{l}\text { The MySQL database must be configured to allow for large } \\
\text { packets in order for the uploaded paper files to be stored } \\
\text { within the database. }\end{array}$ \\
\hline \hline Rule Set & $\begin{array}{l}\text { 1. Open the my.ini (or my.cnf in Linux) configuration file, which } \\
\text { is located in the installation directory of MySQL. } \\
\text { 2. Check that the max_packet_allow property is set to a number } \\
\text { that is big enough to store the largest paper file that } \\
\text { may be submitted. }\end{array}$ \\
\hline
\end{tabular}

Table 6.26: The definition of the JdbcDriverVersion constraint with MySql used as the database server and MySQL Connector/J used as the JDBC driver.

\subsubsection{PostgreSQL}

Selecting the PostgreSQL database and the PostgreSQL JDBC driver results in the WitanWebPostgreSql configuration, which is derived from the WitanWedBase configuration (see Section 6.3).

The PostgreSqlAssembly is created by replacing the Database and JdbcDriver virtual components in DatabaseAssembly (see Section 6.3.14) with the PostgreSql and PostgreSqlJdbcDriver concrete components (see Figure 6.36).

The decision to use these components within the Witan Web system allows for the JdbcDriverVersion constraint, which had previously been declared in Section 6.3.14, to be defined. The definition of this constraint is shown in Table 6.27. 


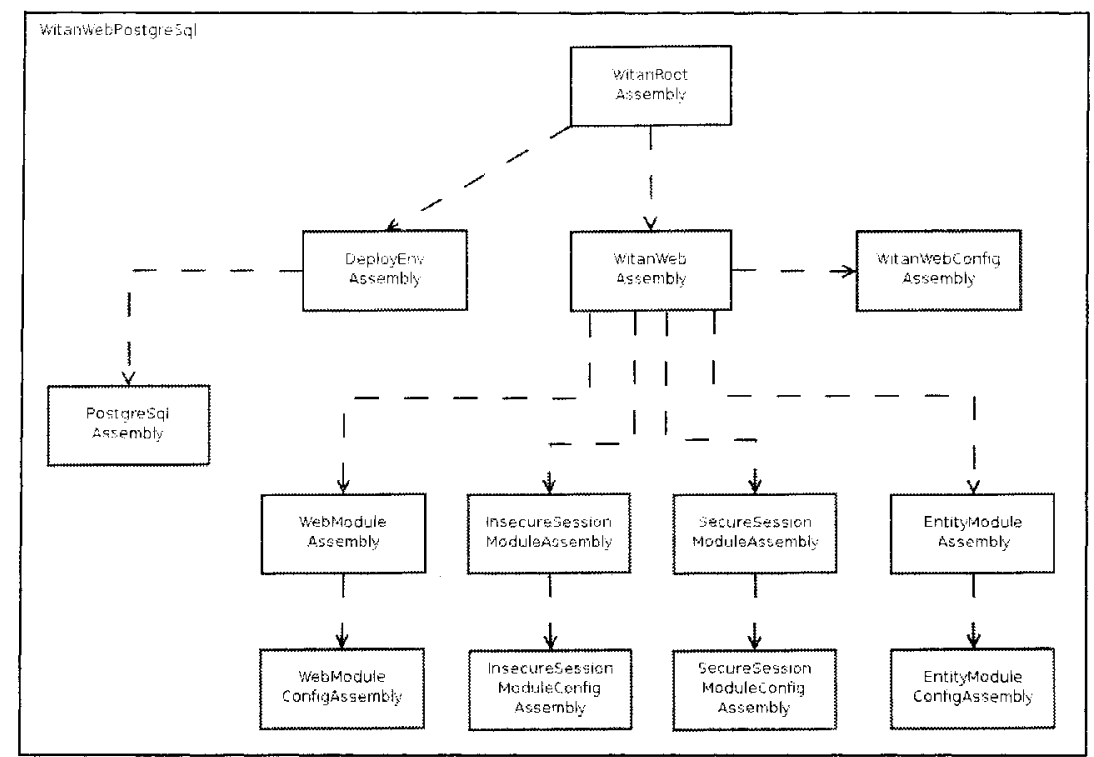

Figure 6.35: The WitanWebPostgreSql configuration, which uses PostgreSql as the database server.

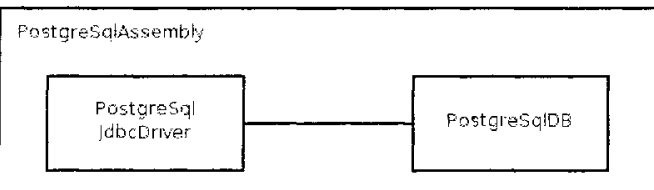

Figure 6.36: The PostgreSqlAssembly assembly. The PostgreSqlJdbcDriver and PostgreSqlDB concrete components in this assembly replace the JdbcDriver and DatabaseServer virtual components, respectively, in DeployEnvAssembly (see Figure 6.14). 


\begin{tabular}{|l|l|}
\hline Identifier & JdbcDriverVersion \\
\hline Description & $\begin{array}{l}\text { The JDBC driver must be the proper version in order to } \\
\text { communicate with the database server. }\end{array}$ \\
\hline \hline Rule Set & $\begin{array}{l}\text { 1. The version of the PostgreSql JDBC driver must be greater } \\
\text { than or equal to the version of the PostgreSql database } \\
\text { server. }\end{array}$ \\
\hline
\end{tabular}

Table 6.27: The definition of the JdbcDriverVersion constraint when using PostgreSql and the PostgreSql JDBC driver as the database server and JDBC driver, respectively.

\subsubsection{Results for Selecting Different Databases}

There were very few constraints discovered as a result of selecting a database server and JDBC driver to be used within the Witan Web system.

In both of the cases presented above, the JdbcDriverVersion constraint, which had been previously declared, was defined. The definition of this constraint required checking that the JDBC driver that had been selected was compatible with the chosen database server.

A unique constraint was identified when the MySQL database server was selected as it had to be configured to allow for large packets. This was required in order to permit the uploaded paper files to be stored within the database.

\subsection{Witan Web Deployable Configurations}

Once all of the virtual components in the base configuration have been replaced with concrete or composite components, it is possible to define a set of deployable configurations. A deployable configuration is created by selecting the set of concrete and composite components that will bo used to deploy an instance of the Witan Web system.

Each deployable configuration will result in a different set of constraints, depending upon which components are selected to be included in the deployed system. As well, all constraints specified within a deployable configuration must be declared and 
defined. This is required in order to verify that all constraints have been properly satisfied within the deployed system.

This section presents two deplovable configurations that have been defined for the Witan Web system in this case study. The deployable configurations have been created using the specializations defined in the previous sections. The set of products used in the two deployable configurations include:

1. JBoss v3 is used as the J2EE application server, MySQL and MySQL Connector/J are used as the database server and JDBC driver, respectively, and the Velocity view is used as the view within the web module (see Section 6.7.1).

2. JBoss $\mathrm{v} 4$ is used as the J2EE application server, PostgreSQL and the PostgreSQL JDBC driver are used as the database server and JDBC driver, respectively, and the JSP view is used as the view within the web module (see Section 6.7.2).

In both deployable configurations, the NRC SMTP server is used as the email server required for sending email messages, and the Sun Java Runtime Environment v1.4 is used as the Java Runtime Environment required by the application server.

Each of these deployable configurations are described in the following sections. Also, a sample of the constraints specified within each deployable configuration is presented.

\subsubsection{Velocity/JBoss3/MySql Deployable Configuration}

The WitanWebJBoss3MySqlVelocityDC deployable configuration is shown in Figure 6.37 .

This deployable configuration is defined by selecting the following components:

- The Velocity view is used as the view within the web module. This replaces the View virtual component, which is located in the WebModuleAssembly assembly (see Section 6.3.11), with the VelocityView composite component in the VelocityWebModuleAssembly assembly (see Section 6.4.2). The VelocityViewAssembly 


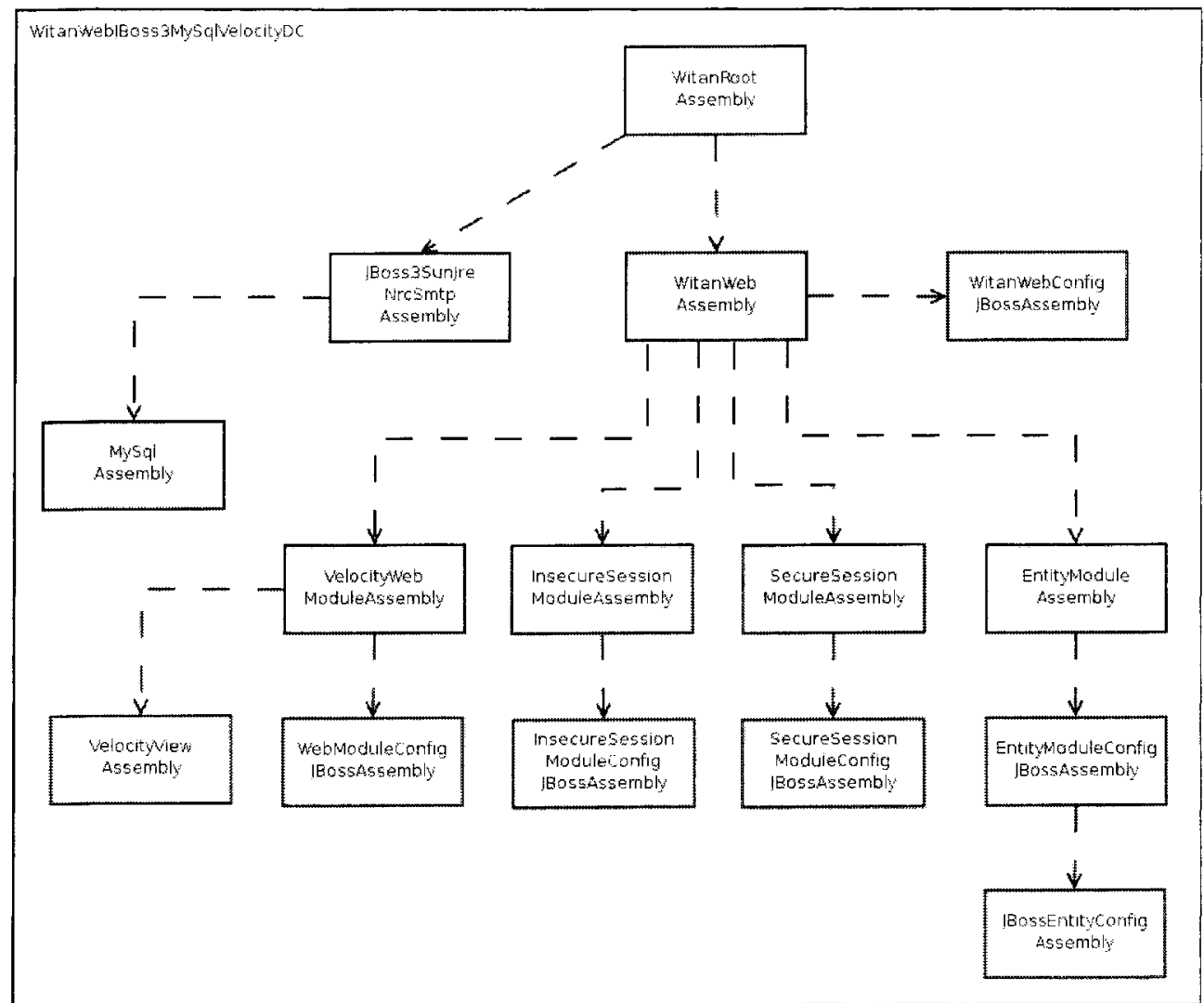

Figure 6.37: The WitanWebJBoss3MySqlVelocityDC deployable configuration. 
is the decomposition assembly of the Velocity View composite component that contains the components required within the Velocity view (see Figure 6.19).

- MySQL and MySQL Connector/J are used as the database scrver and JDBC driver, respectively. This replaces the DatabaseServer and JdbcDriver virtual components, which are located in the DatabaseAssembly (see Section 6.3.14), with the MySqlDB and MySqlConnectorJJdbcDriver concrete components, respectively, in the MySqlAssembly (see Section 6.6.1).

- JBoss v3 is used as the J2EE application server. This replaces the J2EEAppServer virtual component, which is located in the DeployEnvAssembly assembly (see Section 6.3.13), with the JBoss3 concrete component in the JBoss3DeployEnvAssembly (see Section 6.5.1).

- The NRC SMTP server is used as the email server. This replaces the EmailServer virtual component, which is located in the DeployEnvAssembly assembly (see Section 6.3.13), with the NrcSmtpServer concrete component in the JBoss3SunJreNrcSmtpAssembly assembly (see Figure 6.38).

- The Sun Java Runtime Environment is used as the Java Runtime Environment. This replaces the Jre virtual component, which is located in the DeployEn$v A s s e m b l y$ assembly (see Section 6.3.13), with the SunJre concrete component in the JBoss3SunJreNrcSmtpAssembly assembly (see Figure 6.38).

Using the NRC SMTP server and the Sun Java Runtime Environment with the JBoss v3 application server results in the JBoss3SunJreNrCSmtpAssembly assembly, as shown in Figure 6.38. This assembly is derived from the JBoss3DeployEnvAssembly assembly (see Section 6.5.1) by replacing the Jre and EmailServer virtual components with the SunJre and NrcSmtpServer concrete components, respectively.

\section{Constraints}

Selecting the above mentioned products to be used within the Witan Web system results in a large number of constraints being identified, which are related to using the selected products together in the system. 


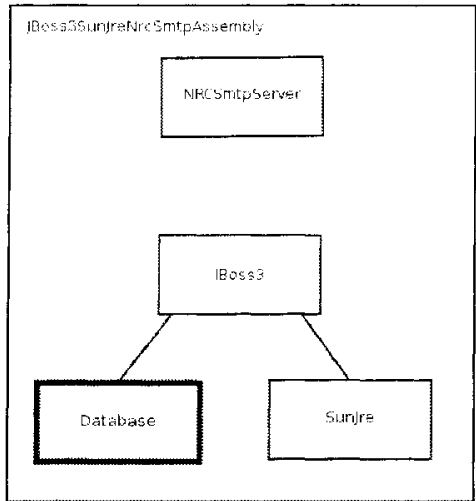

Figure 6.38: The JBoss3SunJreNrCSmtpAssembly assembly. The NrCSmtpServer and SunJre concrete components in this assembly replace the EmailServer and JavaRuntime virtual components, respectively, in JBoss3DeployEnvAssembly (see Figure $6.22)$.

Many of the constraints are related to configuring the application server to communicate properly with the database. This includes configuring a datasource within the JBoss v3 application server for the MySQL database, and ensuring that the MySQL Connector/J JDBC driver is installed in a location where it can be loaded within JBoss v3. These constraints are associated with the (JBoss3, Database) dependency, and are shown in Table 6.28.

Additional constraints, which are associated with the (WitanWeb, DeployEnv) dependency, are also related to configuring the Witan Web software product to work with the combination of JBoss v3 and MySQL. These constraints include overriding the default mappings for the table names created within the database in order to prevent conflicts with the reserved words of the MySQL datbase. The default JDBC data type mappings for certain CMP fields must be overriden to define an SQL type that will allow for large text blocks to be stored within the database. A sample of these constraints is shown in Table 6.29.

In order for the Witan Web product to be able to send email notifications to users throughout the conference management process, the Witan Web product must be configured to work with the NRC SMTP server, which has been selected as the 


\begin{tabular}{|c|c|}
\hline Identifier & ConfiguredDataSource \\
\hline Description & $\begin{array}{l}\text { A data source must be configured within the application server. } \\
\text { This data source establishes a reference to the database, which } \\
\text { is used by the Witan Web product for persisting data. }\end{array}$ \\
\hline Rule Set & $\begin{array}{l}\text { 1. Go to the }<\text { jboss } 3 \text { _home }>/ \text { server/default/deploy directory, } \\
\text { where }<\text { jboss } 3 \text { home }>\text { is the installation directory of } \\
\text { JBoss v3. } \\
\text { 2. Ensure that the mysql-ds.xml configuration file exists. } \\
\text { 3. Open the mysql-ds.xml configuration file. } \\
\text { 4. Check that there exists a local-tx-datasource element with } \\
\text { the jndi-name element set to <conference_name }>\text { DS, where } \\
<\text { conference_name }>\text { is the name of the conference used when } \\
\text { installing the Witan Web software product. } \\
\text { 5. Ensure that the connection-url is set to: } \\
\text { jdbc:mysql://<host_name }>\text { : }<\text { db_port }>/<\text { db_name }> \\
\text { where: } \\
<\text { host_name }>\text { is the host name that the MySQL server is running on } \\
<\text { db_port }>\text { is the port that the MySQL server is listening on } \\
<\text { db_name }>\text { is the name of the database used for storing the data } \\
6 \text {. Ensure that the driver-class element is set to: } \\
\text { com.mysql.jdbc.Driver } \\
\text { 7. Ensure that the user-name element is set to a valid user name } \\
\text { the database. } \\
8 \text {. Ensure that the password element is set to the proper password } \\
\text { for the specified user. }\end{array}$ \\
\hline$\overline{\text { Identifier }}$ & InstalledJdbcDriver \\
\hline Description & $\begin{array}{l}\text { The JDBC driver must be installed in a location where it can be } \\
\text { loaded by the application server. }\end{array}$ \\
\hline Rule Set & $\begin{array}{l}\text { 1. Go to the }<\text { jboss3_home }>/ \text { server/default/lib directory, where } \\
<\text { jboss } 3 \text { home }>\text { is the installation directory of JBoss v3. } \\
\text { 2. Ensure that the MySQL Connector/J JDBC driver JAR file exists. }\end{array}$ \\
\hline
\end{tabular}

Table 6.28: A sample of the constraints associated with the (JBoss3. Database) dependency within the WitanWebJBoss3MySqlVelocityDC deployable configuration. 


\begin{tabular}{|c|c|}
\hline Identifier & UserBeanTableMapping \\
\hline Description & $\begin{array}{l}\text { The table name to be used for the User bean must be set to } \\
\text { WitanUser in order to avoid conflict with the 'user' reserved } \\
\text { keyword in the MySQL database. }\end{array}$ \\
\hline Rule Set & $\begin{array}{l}\text { 1. Open the jbosscmp-jdbc.xml deployment descriptor of the } \\
\text { entity module. } \\
\text { 2. Locate the definition of the User entity bean. } \\
\text { 3. Check that the value of the table-mapping element is set } \\
\text { to WitanUser. }\end{array}$ \\
\hline Identifier & "BiographyField TypeMapping \\
\hline Description & $\begin{array}{l}\text { The type for the biography CMP field of the Paper bean must } \\
\text { be mapped for the default JDBC type of VARCHAR to the SQL type } \\
\text { TEXT in the jbosscmp-jdbc.xml deployment descriptor of the } \\
\text { entity module. }\end{array}$ \\
\hline Rule Set & $\begin{array}{l}\text { 1. Open the jbosscmp-jdbc.xml deployment descriptor of the entity } \\
\text { module. } \\
\text { 2. Locate the definition of the Paper entity bean. } \\
\text { 3. Check that there exists a cmp-field element with the } \\
\text { field-name element set to biography. } \\
\text { 4. Check that the jdbc-type element is set to VARCHAR. } \\
\text { 5. Ensure that the sql-type element is set to TEXT. }\end{array}$ \\
\hline
\end{tabular}

Table 6.29: A sample of the constraints associated with the (WitanWeb, DeployEnv) dependency that are required to allow for the Witan Web product to work with the JBoss v3 application server and the MySQL database. 
outgoing mail server. The constraints identified relating to this relationship are associated with the (WitanWeb, DeployEnv) dependency. A sample of these constraints is shown in Table 6.30 .

\subsubsection{JSP/JBoss4/PostgreSQL Deployable Configuration}

The WitanWebJBoss4PostgreSqlJspDC deployable configuration is shown in Figure 6.39 .

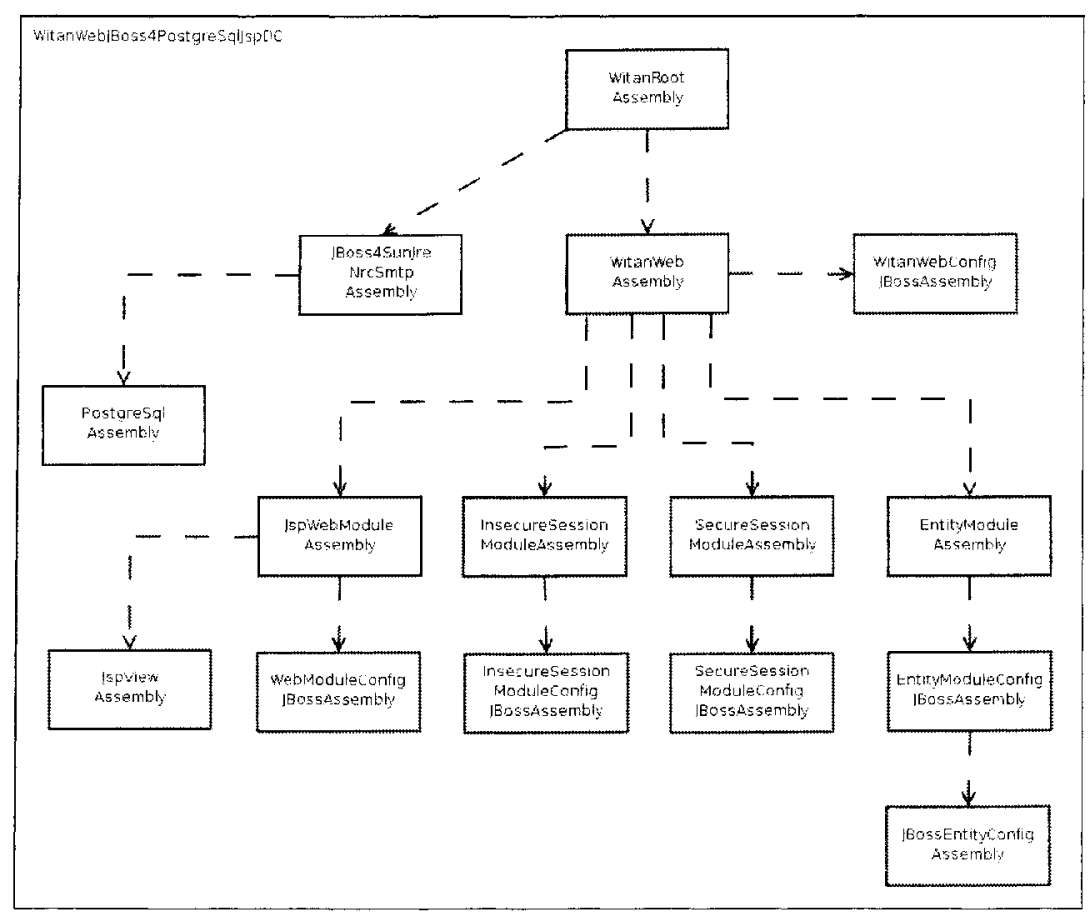

Figure 6.39: The WitanWebJBoss4PostgreSqlJspDC deployable configuration.

This deployable configuration is created by selecting the following components:

- The JSP view is used as the view within the web module. This replaces the View virtual component, which is located in the WebModuleAssembly assembly (sec Section 6.3.11), with the Jsp View composite component in the Jsp WebModuleAssembly assembly (see Section 6.4.1). The JspVieuAssembly is the 


\begin{tabular}{|c|c|}
\hline Identifier & NRCSMTPSeverHost Name \\
\hline Description & $\begin{array}{l}\text { The host name of the NRC SMTP server must be configured } \\
\text { within the web.xml deployment descriptor of the web } \\
\text { module within the Witan Web software product. }\end{array}$ \\
\hline Rule Set & $\begin{array}{l}\text { 1. Open the web.xml deployment descriptor of the web } \\
\text { module. } \\
\text { 2. Locate the env-entry element with the } \\
\text { env-entry-name element set to } \\
\text { settings/outgoingMailServer. } \\
\text { 3. Ensure that the env-entry-value element is set to the } \\
\text { host name of the the NRC SMTP server. } \\
\text { 4. Ensure that the env-entry-type element is set to } \\
\text { java.lang.String. }\end{array}$ \\
\hline Identifier & " NRCSMTPSeverUserName \\
\hline Description & $\begin{array}{l}\text { The user name of a valid user of the NRC SMTP server must be } \\
\text { configured within the web.xml deployment descriptor of } \\
\text { the web module within the Witan Web software product. }\end{array}$ \\
\hline Rule Set & $\begin{array}{l}\text { 1. Open the web.xml deployment descriptor of the web } \\
\text { module. } \\
\text { 2. Locate the env-entry element with the } \\
\text { env-entry-name element set to } \\
\text { settings/outgoingMailServerUserName. } \\
\text { 3. Ensure that the env-entry-value element is set to a } \\
\text { valid user name within the NRC SMTP server. } \\
\text { 4. Ensure that the env-entry-type element is set to } \\
\text { java.lang.String. }\end{array}$ \\
\hline
\end{tabular}

Table 6.30: A sample of the constraints associated with the (WitanWeb, DeployEnv) dependency that are required to allow for the Witan Web product to work with the NRC SMTP server. 
decomposition assembly of the Jsp View composite component that contains the components required within the JSP view (see Figure 6.17).

- PostgreSQL and the PostgreSQL JDBC driver are used as the database server and JDBC driver, respectively. This replaces the DatabaseServer and JdbcDriver virtual components, which are located in the DatabaseAssembly (see Section 6.3.14), with the PostgreSqlDB and PostgreSqlJdbcDriver concrete components, respectively, in the PostgreSqlAssembly (see Section 6.6.2).

- JBoss v4 is used as the J2EE application server. This replaces the J2EEAppServer virtual component, which is located in the DeployEnvAssembly assembly (see Section 6.3.13), with the JBoss4 concrete component in the JBoss4DeployEnvAssembly (see Section 6.5.2).

- The NRC SMTP server is used as the email server. This replaces the EmailServer virtual component, which is located in the DeployEnvAssembly assembly (see Section 6.3.13), with the NrcSmtpServer concrete component in the JBoss4SunJreNrcSmtpAssembly assembly (see Figure 6.40).

- The Sun Java Runtime Environment is used as the Java Runtime Environment. This replaces the Jre virtual component, which is located in the DeployEnvAssembly assembly (see Section 6.3.13), with the SunJre concrete component in the JBoss4SunJreNrcSmtpAssembly assembly (see Figure 6.40).

Using the NRC SMTP server and the Sun Java Runtime Environment with the JBoss v4 application server results in the JBoss4SunJreNrCSmtpAssembly assembly, as shown in Figure 6.40. This assembly is derived from the JBoss 4 DeployEnvAssembly assembly (see Section 6.5.2) by replacing the Jre and EmailServer virtual components with the SunJre and NrcSmtpServer concrete components, respectively.

\section{Constraints}

Deciding to use the above mentioned products within the Witan Web system results in a large number of constraints being identified. These constraints are related to using the selected products together within the system. 


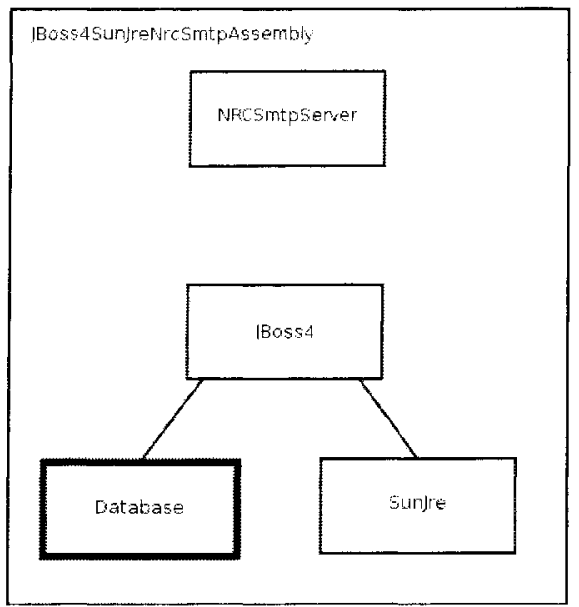

Figure 6.40: The JBoss4SunJreNrCSmtpAssembly assembly. The NrcSmtpServer and SunJre concrete components in this assembly replace the EmailServer and JavaRuntime virtual components, respectively, in JBoss4DeployEnvAssembly (see Figure $6.24)$.

Many of the constraints are related to configuring the application server to communicate properly with the database. This includes configuring a datasource within the JBoss v4 application server for the PostgreSQL database, and ensuring that the PostgreSQL JDBC driver is installed in a location where it can be loaded within JBoss v4. A sample of these constraints are associated with the (JBoss4, Database) dependency, and are shown in Table 6.31.

In order for the Witan Web product to be able send email notifications to users throughout the conference management process, the Witan Web product must be configured to work with the NRC SMTP server, which has been selected as the outgoing mail server. The constraints identified relating to this relationship are associated with the (WitanWeb, DeployEnv) dependency. A sample of these constraints is shown in Table 6.32 . 


\begin{tabular}{|c|c|}
\hline Identifier & ConfiguredDataSource \\
\hline Description & $\begin{array}{l}\text { A data source must be configured within the application server. } \\
\text { This data source establishes a reference to the database, which } \\
\text { is used by the Witan Web product for persisting data. }\end{array}$ \\
\hline Rule Set & 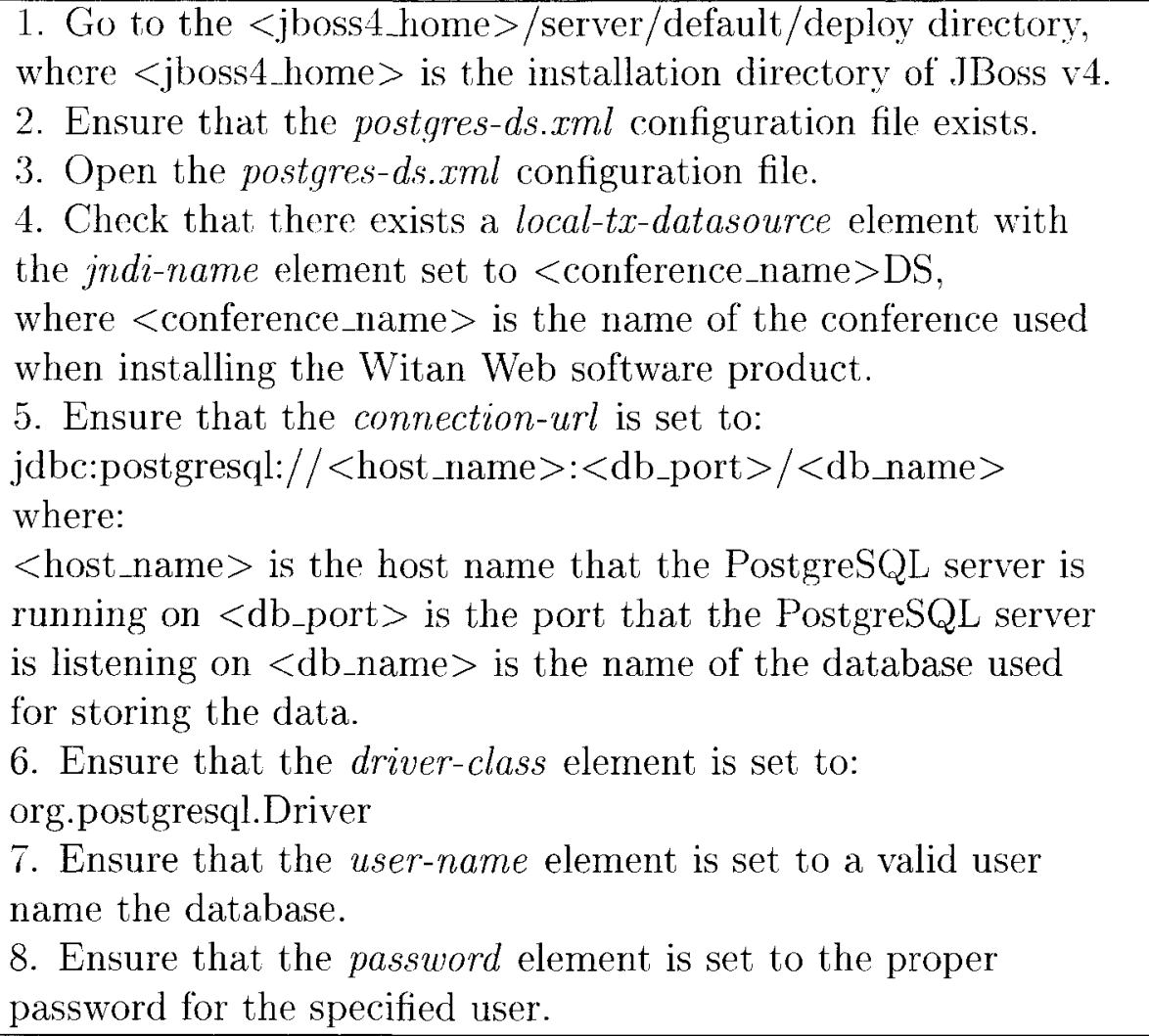 \\
\hline Identifier & InstalledJdbcDriver \\
\hline Description & $\begin{array}{l}\text { The JDBC driver must be installed in a location where it can be } \\
\text { loaded by the application server. }\end{array}$ \\
\hline Rule Set & $\begin{array}{l}\text { 1. Go to the }<\text { jboss } 4 \text { home }>\text { /server/default/lib directory, where } \\
<\text { jboss4_home }>\text { is the installation directory of JBoss } \mathrm{v} 4 \text {. } \\
\text { 2. Ensure that the PostgreSQL JDBC driver JAR file exists. }\end{array}$ \\
\hline
\end{tabular}

Table 6.31: A sample of the constraints associated with the (JBoss4, Database) dependency within the WitanWebJBoss4MySqlVelocityDC deployable configuration. 


\begin{tabular}{|c|c|}
\hline Identifier & NRCSMTPSeverHostName \\
\hline Description & $\begin{array}{l}\text { The host name of the NRC SMTP server must be configured } \\
\text { within the web.xml deployment descriptor of the web } \\
\text { module within the Witan Web software product. }\end{array}$ \\
\hline Rule Set & $\begin{array}{l}\text { 1. Open the web.xml deployment descriptor of the web } \\
\text { module. } \\
\text { 2. Locate the env-entry element with the } \\
\text { env-entry-name element set to } \\
\text { settings/outgoingMailServer. } \\
\text { 3. Ensure that the env-entry-value element is set to the } \\
\text { host name of the the NRC SMTP server. } \\
\text { 4. Ensure that the env-entry-type element is set to } \\
\text { java.lang.String. }\end{array}$ \\
\hline Identifier & NRCSMTPSeverUserName \\
\hline Description & $\begin{array}{l}\text { The user name of a valid user of the NRC SMTP server must be } \\
\text { configured within the web.xml deployment descriptor of } \\
\text { the web module within the Witan Web software product. }\end{array}$ \\
\hline Rule Set & $\begin{array}{l}\text { 1. Open the web.xml deployment descriptor of the web } \\
\text { module. } \\
\text { 2. Locate the env-entry element with the } \\
\text { env-entry-name element set to } \\
\text { settings/outgoingMailServerUserName. } \\
\text { 3. Ensure that the env-entry-value element is set to a } \\
\text { valid user name within the NRC SMTP server. } \\
\text { 4. Ensure that the env-entry-type element is set to } \\
\text { java.lang.String. }\end{array}$ \\
\hline
\end{tabular}

Table 6.32: A sample of the constraints associated with the (WitanWeb, DeployEnv) dependency that are required to allow for the Witan Web product to work with the NRC SMTP server. 


\subsubsection{Results for Different Deployable Configurations}

Much of the work in specifying constraints within the two deployable configurations is related to defining constraints that had previously been declared. All declared constraints must be defined within a deployable configuration so that all constraints may be checked to ensure that they have been properly satisfied.

Since different versions of JBoss were used in both deployable configurations, and JBoss has worked to maintain backward compatibility between versions of the application server, many of the definitions of constraints were similar within the two deployable configurations. However, if a different application server were used, these definitions would be very different.

Using different combinations of products resulted in different sets of constraints being specified. This was evident in the Witan WebJBoss3MySqlVelocityDC deployable configuration, where using the JBoss v3 application server with the MySQL database required that the default table name mappings to be overriden in order to prevent conflicts with certain reserved keywords used in MySQL.

\subsection{Summary of Dependencies and Constraints}

This case study presented a sample of the constraints that were discovered within the Witan Web system while creating the two deployable configurations. The actual number of discovered constraints that were declared and defined were in the order of several hundred. This section summarizes the types of constraints that were discovered at each stage of the case study, and provides insight into the number of identified constraints.

\section{Constraints identified in the base configuration}

The base configuration had the largest number of constraints specified, and these constraints were primarily related to the configuration of each module within the Witan Web product. Some of the types of constraints specified include: 
- Module configurations: All J2EE modules must be configured within the application.xml deployment descriptor of the Witan Web software product.

- EJB definitions: All EJBs must be defined within the appropriate deployment descriptor by configuring the proper interfaces. This resulted in the specification of a single constraint for each EJB contained within the Witan Web product.

- EJB references: All EJB references, local and remote, must be configured within the proper deployment descriptors. Each EJB reference within the Witan Web product resulted in the specification of a constraint.

- EJB QL Queries: All EJB QL queries within the entity beans must be configured within the ejb-jar.xml deployment descriptor of the entity module. Each query resulted in the specification of a constraint.

- EJB transactions: All methods within each EJB must be configured to make use of container managed transactions. This resulted in the specification of a single constraint for each EJB to ensure all methods use transactions.

- Form beans: All form beans within the Servlet controller must be configured within the struts-config.xml configuration file. This resulted in the specification of a single constraint for each form bean.

- Web view actions: All of the actions that are handled within the web view must be configured within the struts-config.xml configuration file. This resulted in the declaration of a single constraint for each action; these constraints must be defined once an implementation of the view is selected.

- Struts plugins: All Struts plugins must be configured within the struts-config.xml configuration file. Each plugin configuration resulted in a single constraint being specified.

- Validation definitions: All validation methods for the forms used in the web view must be defined within the validator.xml configuration file. This resulted in a single constraint being specified for each validation method. 
- Validation rules: All validation rules used within the validation methods must be defined within the validator-rules. $x m l$ configuration file. This resulted in a single constraint being specified for each validation rule.

- Tiles definitions: All Struts tiles must be defined within the tiles-defs.xml configuration file. Each file definition resulted in a single constraint being specified.

\section{Constraints identified while selecting views}

Selecting the JSP and Velocity views to be used within the Witan Web product required that all of the constraints related to the web view actions, which had been declared in the base configuration, be defined. The definition of these constraints for each view involved:

- JSP View: The definition must ensure that each action is properly configured to reflect the JSP page from which the action is invoked, as well as to which JSP page control should be forwarded, upon successful completion of the action.

- Velocity View: The definition must ensure that each action is properly configured to reflect the Velocity page from which the action is invoked, as well as to which Velocity page control should be forwarded, upon successful completion of the action.

The selection of each view resulted in a set of unique constraints being specified which were related to properly configuring the view. In the case of the JSP view, this included ensuring that the tag libraries used are of the proper version. and that the tag libraries are configured within the web.xml deployment descriptor.

In the case of the Velocity view, the proper version of the Velocity template engine must be included within the view, and the template engine must be configured within the web.xml deployment descriptor. As well, the Velocity template engine must be configured to allow for integration with the Struts framework. 


\section{Constraints identified while selecting application servers}

Selecting the JBoss $v 3$ and JBoss v4 application servers to be used in deploying the Witan Web software product resulted in a set of constraints being identified, which were related to the JBoss specific configuration files used within the Witan Web product. The constraints associated with these configuration files included:

- JBoss QL queries: The queries for finder methods must be overriden in the jbosscmp-jdbc.xml configuration file using the JBoss Query Language in instances where the EJB Query Language is not sufficient. This resulted in the specification of a single constraint for each overriden query method.

- Read only methods: All get and value object creation methods within the entity beans must be configured as read only. This resulted in the specification of a single constraint for each set of get methods and value object creation method in each entity bean.

As well, a set of constraints were identified pertaining to the configuration of the application servers in order to allow for the Witan Web product to be deployed and function properly.

\section{Constraints identified while selecting databases}

Selecting the MySQL and PostgreSQL databases resulted in a small set of constraints being specified. These constraints were primarily related to the compatibility between the JDBC driver and the database server.

In the case of MySQL, there was an additional constraint specified pertaining to the configuration of the maximum packet size allowed in order to store uploaded paper files in the database.

\section{Constraints identified within the deployable configurations}

Within the deployable configurations, the emphasis was on defining the constraints that had previously been defined. 
The majority of these constraints pertained to using a particular combination of application server and database. These included:

- Ensuring that the data source is properly configured.

- Ensuring that the JDBC driver is installed in a location where it may be loaded by the application server.

- Overriding the default JDBC data type mappings for Java strings to define an SQL type that is capable of storing large text blocks.

- Overriding the default table name mappings in order to prevent conflicts with reserved keywords in the database.

As well, the constraints associated with using the NRC SMTP server and the Sun Java Runtime Environment were specified.

It is evident that there are a large number of constraints that exist amongst the components within the Witan Web system. This case study has shown how the management of these constraints may be made easier using the research presented in this thesis.

\subsection{Discussion}

Applying the dependency and constraint management process to the Witan Web conference management system presented several issues and topics of interest.

One of the issues encountered was that defining the configurations and specializations for the Witan Web system was a tedious and time consuming process. The tool did not alleviate the burden of this process, as it required that each configuration be manually defined within an XML file. Thus, the definition of configurations proved to be the most difficult aspect of the dependency and constraint management process.

The definition of constraints also proved to be a time consuming process. This was due to the number of constraints that existed within the Witan Web system. The tool assisted with the process by allowing all actors involved with the system to define 
constraints. It also allowed for constraints defined within a particular configuration to be propogated to all specializations of the configuration.

The possibility of defining a set of patterns for creating the configurations and specializations emerged as a topic of interest throughout the case study. Defining a pattern language and a set of patterns for creating configurations should help in the dependency and constraint management process. Throughout the case study, two possible patterns were discovered:

- Separating the assembled product from the deployment environment: In the root assembly of the Witan Web system, a composite component was defined for the Witan Web product, and a composite component was defined for the deployment environment required by the Witan Web product. This allowed for a clear separation of constraints that were discovered between the components within the Witan Web product and the constraints that were discovered between the components used within the deployment environment. It also provided a single dependency between the Witan Web product and the deployment environment with which all constraints identified between the Witan Web product and the deployment environment were associated.

- Separating product implementation from product configuration: When defining the base configuration of the Witan Web system, the components related to the implementation of a product were separated from components related to the configuration information of the product. This was accomplished by defining two composite components: one for the product implementation and one for the product configuration. A dependency was then defined between the components. This allows for constraints related to the implementation of a product to be separated from the constraints related to the configuration of a product.

Another topic of interest identified throughout the case study was related to the classification of constraints. The constraints that were discovered within the Witan Web system may have been categorized, or classified, depending based upon the type of constraint. For example, constraints may be classified according to:

- Product version requirements 
- Product configuration

- Product deployment

This classification of constraints may facilitate searching for constraint information related to a particular aspect of a system, and thus troubleshooting deployments that are not functioning as expected may be made casier.

Addressing the issues, and investigating the topics of interest, discovered throughout this case study is left as future work that may further assist in the management of dependencies and constraints in software systems.

\subsection{Summary}

This chapter presented the case study that was carried out as part of this thesis. The case study applied the research presented in this thesis to the Witan Web conference management system.

The structure of the Witan Web software system was specified by defining the base configuration of the system. This involved defining all of the components and dependencies within the Witan Web system, using virtual components whenever possible. All of the constraints identified within the base configuration were also specified.

Multiple instances of the Witan Web software product were then specified by selecting two different views for the web-based front end of the Witan Web product. One instance of the product used a JSP-based view, and the other used a Velocitybased view. A specialization of the base configuration was created for each of these instances. All of the newly discovered constraints were specified for each of these specializations.

Multiple instances of the Witan Web system were specified by selecting different application servers and databases to be used within the system. Both the JBoss v3 and JBoss v4 application servers were used within the system, and the MySQL and PostgreSQL databases were used within the system. Specializations were created to reflect the sclection of each of these products, and the newly discovered constraints were specified. 
Finally, two separate deployable configurations were presented for the Witan Web system. The first deplovable configuration used the Velocity-based view, JBoss v3 as the application server, and MySQL as the database. The second deployable configuration used the JSP-based view, JBoss v4 as the application server, and PostgreSQL as the database. All of the constraints identified in each of these deployable configurations were specified.

This case study showed that even for a system of moderate size, hundreds of constraints may exist within the system. All of the constraints identified throughout this case study must be satisfied in order for the Witan Web system to function properly.

Depending upon the set of products used within each deployed instance of the Witan Web system, the set of constraints varied. If an organization deploys the Witan Web system using the same set of components as used in one of the deployable configurations presented in this case study, then the constraints associated with the configuration may be referenced, which prevents the organization having to rediscover the constraints.

If an organization deploys the Witan Web system using a different set of components, then the dependency model of the Witan Web system should be updated accordingly. Any overlap in the components selected would allow for the organization to reuse the dependency and constraint information that has already been defined; however, but the dependency model must be updated to reflect the selection of components that have not previously been used, and the newly discovered constraints must specified. This will allow for more deployable configurations to be defined, which may be referenced by other organizations in the future.

Finally, throughout this case study, constraint information was gathered from multiple actors in order to determine the benefit of open collaboration in managing dependencies and constraints. This resulted in dependencies and constraints being identified that were previously unknown to the developers of the Witan Web product. Thus, the open collaboration proved to be beneficial in defining a more complete set of constraints for the two deplovable configurations of the Witan Web system that were presented in this case study. 


\section{Chapter 7}

\section{Conclusion}

\subsection{Context and Motivation}

As the scale and complexity of software systems has increased, there has been a shift in how software products are developed. It has become increasingly common for large software products to be produced by assembling a set of existing software products. In situations where several products exist that provide the same functionality, a decision must be made as to which product is to be used. The ability to choose different products to be integrated into the assembled product makes it possible to produce several instances of the assembled product. Each instance may use a different product to provide the same functionality. This flexibility is important to organizations producing product lines or frameworks, where it is necessary for the product to contain selection points whenever there exist several alternatives that provide the required functionality.

When an assembled software product is shipped, it rarely is capable of running properly out of the box. Typically, a set of services are required by the product in order for the product to be deployed as a functional system. In the simplest case, an operating system is required, but, in more complex cases, additional services such as a database and application server may be required. Again, there may be several products available that provide the same service, and a decision must be made as to which product will be used. Multiple instances of the system may be deployed by 
selecting different products to provide the required services.

Assembling and deploying a software product in this manner results in a large set of dependencies between the products. Each dependency may have several restrictions. or constraints, associated with it that must be satisfied in order for the deployed system to function properly.

The software products used within the assembled product and the deployed system are developed by separate development teams, and in most cases, separate organizations. As a result, the products evolve independently. This evolution results in a set of dependencies and constraints that also evolve.

It is important to properly manage the dependencies and constraints throughout the life cycle of a software product in order to prevent having to rediscover them each time that the product is deployed. Currently, it is the responsibility of the organization providing the product to document and manage the dependencies and constraints, and this is typically done through installation instructions and user guides. However, users of the product may discover additional constraints as they use the product in contexts that were unexpected by the organization providing the product. These constraints may be documented in mailing lists or user groups, or they may not be documented at all. In this case, the constraint must be rediscovered by others that use the product in the similar contexts.

\subsection{Problem Statement}

This thesis addresses the problem of managing the dependencies and constraints in a software product that has been assembled using a set of existing products, where there is variability in the products available, and deployed with a set of required services, which may also be variable, to produce a functional software system.

\subsection{Objectives}

This thesis set out to solve this problem by meeting the following objectives: 
1. Provide a formalism for the application assembly process: The application assembly process is the portion of the software development process that deals with assembling a software product from a set of existing products and deploying the product to produce a functional system. The formalization of this process would provide the context in which software products are assembled and deployed. It would also describe how the large number of dependencies and constraints that exist in assembled software system are discovered and managed.

2. Define a set of requirements for managing constraints in software systems: Using the formalization of the application assembly process, a set of requirements for managing the constraints in a software system could be defined.

3. Develop a formal specification for modeling assembled software systems: A formal specification for modeling assembled software systems could be used as the base for providing tool support to meet the requirements of the constraint management process.

4. Define a process for modeling a system and specifying constraints: Using the formal specification it was possible to define a process for modeling the structure of a software system and specifying constraints as they are discovered.

5. Use open collaboration for managing constraints: In order to prevent having to rediscover constraints throughout the life cycle of a software system, it was believed that open collaboration amongst all actors involved with the system could simplify the constraint management process.

\subsection{Contributions}

This thesis addressed the above stated objectives by providing the following contributions to the field of software engineering: 


\section{Formalization of the application assembly process}

The application assembly process was formalized by defining a set of use cases that describe each of the activities involved in the process. These use cases include:

- Code a software product

- Select software products

- Assemble a software product

- Deploy a software product

- Manage dependencies and constraints

A set of actors that are associated with these use cases was also specified.

\section{Defined requirements for managing constraints}

A set of scenarios was presented that described several situations that may arise when assembling and deploying a software product. The scenarios were based upon the use cases defined in the formalization of the application assembly process. Using these scenarios, a set of requirements for managing dependencies and constraints was defined. These requirements included:

- define dependencies and constraints

- view existing constraints

- search existing constraints

- update existing constraints

- verify deployments

- distribute dependencies and constraints

- provide open collaboration 


\section{Formal specification for modeling assembled software systems}

A formal specification for modeling the structure of a software system was developed. The formal specification allowed for the software products within the system to be modeled, as well as the dependencies between the products. It is also possible to model selection points within the system whenever there exists more than one product that provide the same functionality.

Different instances of a system may be modeled by specializing an existing model. Specializing a model involves deciding upon a particular product to be used for each of the defined selection points. Once all of the selection points have been assigned a product, the model represents a deployable system.

The formal specification was meant to be used as a base for defining a process and building tools to meet the requirements of the constraint management process.

\section{Process for modeling the system structure and specifying constraints}

A process for modeling the structure of a software system and specifying constraints was defined, using the formal specification as a base. The process includes the following activities:

- Define the high level structure of the software system by specifying as many selection points within the model as possible. This allows for the greatest amount of flexibility when deciding which products to use in the deployed system.

- Specialize the high level system model by replacing the defined selection points with the products that have been selected to be included within the system.

- Specify constraints as they are discovered. This prevents constraints from having to be rediscovered in the fiture.

- Verify that all of the constraints have been satisfied in a deployed system. This is done manually by checking that each of the defined constraints within the system has been properly satisfied. 


\section{Tool support allowing for open collaboration in managing constraints}

A tool was developed that provided support for open collaboration amongst all actors involved with a software system in the constraint management process. The support for open collaboration was provided by implementing the tool using a client-server architecture.

The server acts as a repository that is responsible for storing the system models, as well as the constraints that have been specified within the system.

The client is a web-based application that runs within a standard web browser. It allows for all actors involved with a system to add and remove constraints, as well as update existing constraints.

\section{Applied the constraint management process to the Witan Web system}

In order to demonstrate how the research presented in this thesis is applied in a real world situation, a case study was presented that applied the constraint management process to the Witan Web conference management system.

The case study explained how the Witan Web software product was assembled, and several instances of the product were presented by selecting different views to be used. The product was then deployed using different databases and application servers to demonstrate how multiple instances of the system may be defined.

All of the constraints that were discovered within the Witan Web system were also specified throughout the case study.

\subsection{Future Work}

There are several issues related to this research that may be addressed in future work. These issues include:

\section{Use UML for the formal specification}

The formal specification presented in this thesis was defined using a mathematical model. It may be possible to redefine this specification using the Unified Modeling 
Language (UML), with the Object Constraint Language (OCL) being used to define the rules governing how the system models must be defined. As well, it may be possible to use UML as a replacement to the graphical representation of each element defined in the formal specification.

\section{Automatic dependency discovery}

This research required that all dependencies be explicitly defined as they were discovered. It may be possible to provide a mechanism for automatically discovering dependencies within a software system. This automatic discovery of dependencies must focus on identifying dependencies that exist not only at the implementation interface level of software products but also at the deployment and configuration level. For example, a software product may depend on the existence of a configuration file in order to function properly. This dependency is not defined at the interface level, but it must be captured during the dependency and constraint management process.

This process may require different sets of input in order to automatically generate the dependency model. One type of input that may be used is a system design model that has been created using either UML or another architecture description language (ADL). This may be used to determine the dependencies between components that are related to functional aspects of the system.

Other forms of input would have to be provided in order to generate the dependencies related to the components representing the configuration information.

\section{System structure validation}

The formal specification defined within this thesis does not provide any capability for validating the structure of a system based upon the software products and dependencies defined within the system. For example, if it is impossible for two products to be used within a system, the formal specification does not provide a mechanism for detecting this situation. It may be possible to define a procedure for automatically validating the configurations defined for a system. 


\section{Formal language for constraints}

This thesis did not attempt to define a formal language for specifying constraints. It may be possible to either define a new constraint language or extend an existing constraint language, such as the Object Constraint Language (OCL), that may be used to express all possible types of restrictions that may exist within a software system.

In the case where a single language can not be defined to represent all types of constraints, the possibility of using multiple languages for defining constraints may also be investigated. Even if it is not possible to define all of the constraints within a system using a formal language, the more constraints that are formally defined, the greater the portion of the verification process that may be automated.

\section{Classification of constraints}

As discussed in the case study (see Chapter 6), it may be possible to define a set of categories that may be used to classify the constraints within a software system. This classification of constraints may help provide guidance in the dependency and constraint management process as it would specify the different types of constraints to be identified while defining constraints within the system. Also, classifying constraints may make it easier to identify the cause of a problem in a deployed system that is not functioning properly as particular problems may be associated with constraints of a certain type, or classification.

\section{Defining dependency model patterns}

It may be possible to define a pattern language, and a set of patterns, to assist with the creation of a dependency model. Chapter 6 discussed several possible patterns that emerged throughout the Witan Web case study. Providing a formal definition for these patterns, as well as for any other patterns that may be discovered, would allow for dependency models to be created more easily and be more comprehensible. 


\section{Extension of tool support}

The tool developed as part of this research provided support for defining configurations and specializations, as well as adding, removing and updating constraints. The tool also provided support for open collaboration amongst all actors involved with a software system. It may be possible to extend the existing tool to provide a mechanism for modeling the structure of a software system using the formal specification defined within this thesis. Also, it may be possible to intcgrate the tool with a Software Configuration Management (SCM) tool that could be used to manage the evolution of the system structure and constraint information.

\subsection{Conclusions}

Several conclusions have been drawn from the research presented in this thesis:

\section{Model vs. Reality}

When managing dependencies and constraints, it is very difficult to automatically apply the information defined in the formal model of a system to the actual installed system. This concept of going from model to implementation is addressed within the Model Driven Architecture (MDA) [21] approach to developing software systems.

However, in the context of managing dependencies and constraints, this approach suffers from a major drawback: constraints exist in many forms. A constraint may be related to the version of a product, the existence of a particular file system, or the value of a configuration property, amongst other things. As a result, it is very difficult to define a formal language for specifying constraints. Without a formal language for defining constraints, it is difficult to automatically apply the constraint information contained in a model to that which exists in reality.

\section{Feasibility of modeling dependencies in complex systems}

Complex software systems typically contain a large number of dependencies, and modeling these dependencies can be a difficult task: however, it is not infeasible. 
Typically there exists some form of documentation that may be used to assist in the dependency modeling process, whether it be a formal model or installation documentation. Using the existing information, the process of creating a dependence model is simplified.

The modeling of dependencies in software systems that are assembled using existing products may be further simplified in the case where each product included in the system has a previously defined dependence model. The existing dependence models could then be integrated into the dependence model of the assembled software system, thus reducing the workload and easing the process.

\section{Usefulness of open collaboration in discovering constraints}

Applying the concept of open collaboration to the context of managing dependencies and constraints is a useful approach. The Beagle [3] project displayed this usefulness by making a wiki [47] available to help manage the dependencies and constraints. The wiki allowed all members of the Beagle community to partake in the dependency and constraint management process, which resulted in up-to-date information pertaining to the installation requirements of the Beagle tool.

The usefulness of open collaboration was also realized throughout the case study as several dependencies and constraints were discovered that had previously been unknown to the developers of the product. This showed that more constraints can be identified and documented by allowing all actors involved with a system to assist in the dependency and constraint management process, which prevents having to rediscover the constraints each time the system is deployed.

\subsection{Discussion}

This thesis addressed the problem of managing dependencies and constraints within a software system. This was shown to be a difficult task when assembling a software product using existing products, and deploying the assembled product along with the services required to produce a functional system. 
The dependency and constraint management process presented in this thesis should be integrated into the software development process. This would prove to be beneficial to an organization as the system structure and constraint information may be continuously updated throughout each iteration of the development process. This integration may be carried out in the following manner:

- The system structure could be modeled during the analysis and design stages

- The constraints could be specified during the coding, assembly, testing, and deployment stages

Relying upon open collaboration amongst all actors involved with the system increases the chances of discovering constraints that may not have been known to the developers of the system. As well, it decreases the likelyhood that constraints will need to be rediscovered each time that a system is deployed.

The tool developed in this thesis may be used to help manage the constraints within a system. In the future, the tool could be extended to provide improved support for sharing the system structure and constraint information across organizations. Also, a tool could be developed to assist in the modeling of the system structure.

It is believed that this thesis provides a solid foundation to approaching the dependency and constraint management process. Future work may improve upon the research presented here by providing a formal language for defining constraints, and this could assist in performing automatic verification of a deployed system. 


\section{Appendix A}

\section{Dependency and Constraint Management Tool}

A tool for managing dependencies and constraints within a software system was developed as part of this research. The tool uses the formal specification defined in Chapter 4 as a base, and it focuses on providing the functionality to allow for open collaboration amongst all of the actors in the dependency and constraint management process.

The tool uses a client-server architecture with the server acting as a repository storing all of the dependency and constraint information defined for a system, and the client allows for the users to access and modify the information stored on the server. The client-server architecture was chosen as a means of implementing the functionality of the open collaboration requirement.

The client is implemented as a web-based application, and users access the client using a standard web browser. Users are able to define and specialize configurations. Constraints may also be added, removed and updated within cach configuration. The client does not use the graphical representation of elements within the formal specification; instead, it is XML based, and configurations are created using a valid XML document that adheres to a specific Document Type Definition (DTD).

Section A.1 describes the implementation details of the tool. This section explains the different technologies used to develop the various layers of the tool. 
Section A.2 describes how the tool is used to manage the dependencies and constraints within a software system. This includes the process of defining configurations, specializing configurations, and adding, removing and updating constraints.

Section A. 3 outlines several fcatures and improvements that may be implemented within the tool in order to make it a more viable tool to be included within the development environment and support documentation for software products.

\section{A.1 Tool Implementation}

The dependency and constraint management tool was developed using a client-server architecture. The client is implemented as a web-based application, which is accessible through a standard web browser.

The server is implemented as a repository, which is responsible for maintaining the dependency and constraint information. On the server side, all of the dependency and constraint information is persisted in a database (Figure A.1).

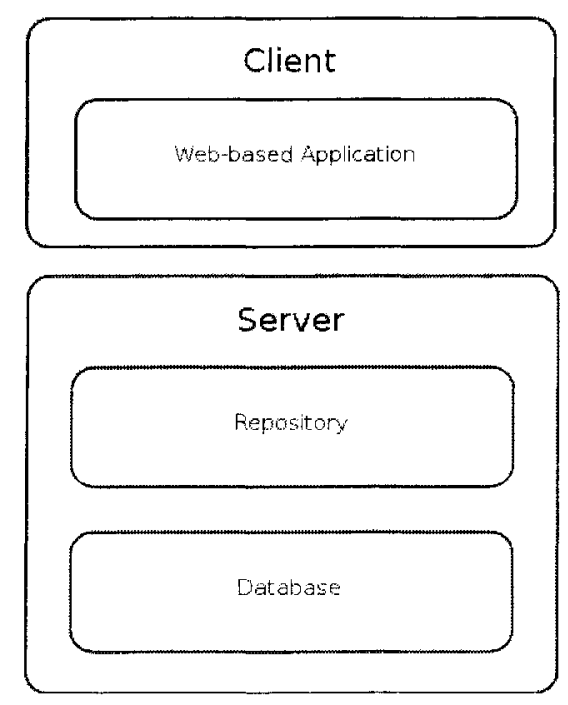

Figure A.1: The architecture of the dependency and constraint managment tool. 


\section{A.1.1 Implementation details}

The dependency and constraint management tool was developed as a Java 2, Enterprise Edition (J2EE) application, and it used several technologies to implement the different tiers of the application. An overview of the technologies used within the tool is shown in Figure A.2.

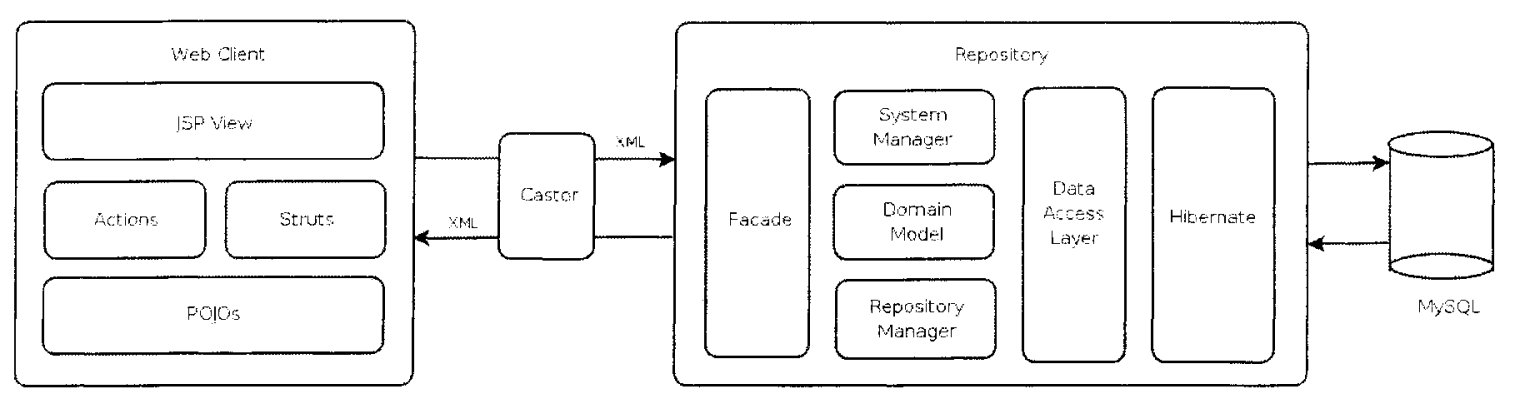

Figure A.2: The implementation of the dependency and constraint managment tool.

The web client was developed using a Model-View-Controller (MVC) architecture, and it was implemented with the following technologies:

- View: The view, or presentation layer, was implemented using JavaServer Pages (JSP) [17]. The JSP Standard Tag Library (JSTL) [16] was used to provide easy access to JavaBean properties, as well as conditional and control logic. This allowed for the presentation layer to be implemented without requiring Java source code to be embedded in the JSP pages.

- Controller: The controller was implemented as a Java Servlet, and it used the Apache Struts framework [37] to provide common functionality such as registering actions and configuring the pages to be rendered upon successful completion of an action.

- Model: The model was implemented using a set of JavaBeans, or Plain Old Java Objects (POJOs). The use of JavaBeans allowed for easy integration with the JSP pages, as well as with the server repository. 
The server repository was developed using a service facade to provide a single point of entry to the repository from the web client. The facade defines the set of methods that may be invoked on the server to manipulate the dependency and constraint information stored within the repository.

The facade encapsulates the implementation of the data access layer, which is responsible for persisting the data contained within the domain model. The data access layer used the Hibernate Object-Relational Mapping (ORM) framework [9] to assist in mapping the object oriented domain model to the relational data model supported by the database.

The domain model portion of the server is the same as the model of the web client, and for this reason the domain model was implemented as a separate library that could be included in both the client and the server. The data contained within the domain model is serialized into an XML format using the Castor XML serialization library [38] so that the data may be easily transmitted from client to server, and vice versa.

The data is persisted within the repository using a relational database management system. The MySQL database server was used within the tool; however, the database may be easily substituted with another product, if necessary.

\section{A.2 Tool Usage}

The web client is accessed by opening a web browser and pointing it at the location of the server hosting the client. Upon startup, a list of all of the systems stored in the repository is displayed (Figure A.3). Within the context of the tool, a system is analogolous with a specialization scheme, as it is defined in the formal specification. A system consists of the base configuration and all specializations that have been defined for a particular software system.

From the start page, it is possible to create a new system or view an existing system. 


\section{5rstges:}

\section{System list}

$$
\text { - ypetstore }
$$

- Witanweb

[ Create ]

Figure A.3: The client view of all systems stored within the repository.

\section{A.2.1 Create a New System}

Choosing to create a new system will display a page containing a form prompting the user to enter a name for the system, a description, and the definition of the base configuration for the system (Figure A.4 and Figure A.5).

The name provided for the system must be unique amongst all other systems stored within the repository. The description should used to give a detailed explanation of the system.

The base configuration is defined in an XML format that must adhere to the configuration_1_0.dtd Document Type Definition (DTD) (see Section A.4).

\section{A.2.2 System View}

Selecting a system from the list presented on the start page will display the system view (Figure A.6). The system view lists all of the configurations that have been defined within the specialization scheme of the system.

The nesting of the configurations represents the specialization relationship between two configurations. For example, the configuration xPetstore-Jboss is a spocialization of the configuration xPetstore-Base (Figure A.6). 


\section{Create System}

\section{MAME}

Flease enter a name for the system. This name must be unique amongst all systems existing in this repository.

\section{DESCRIPTION}

Please enter a description for the system.

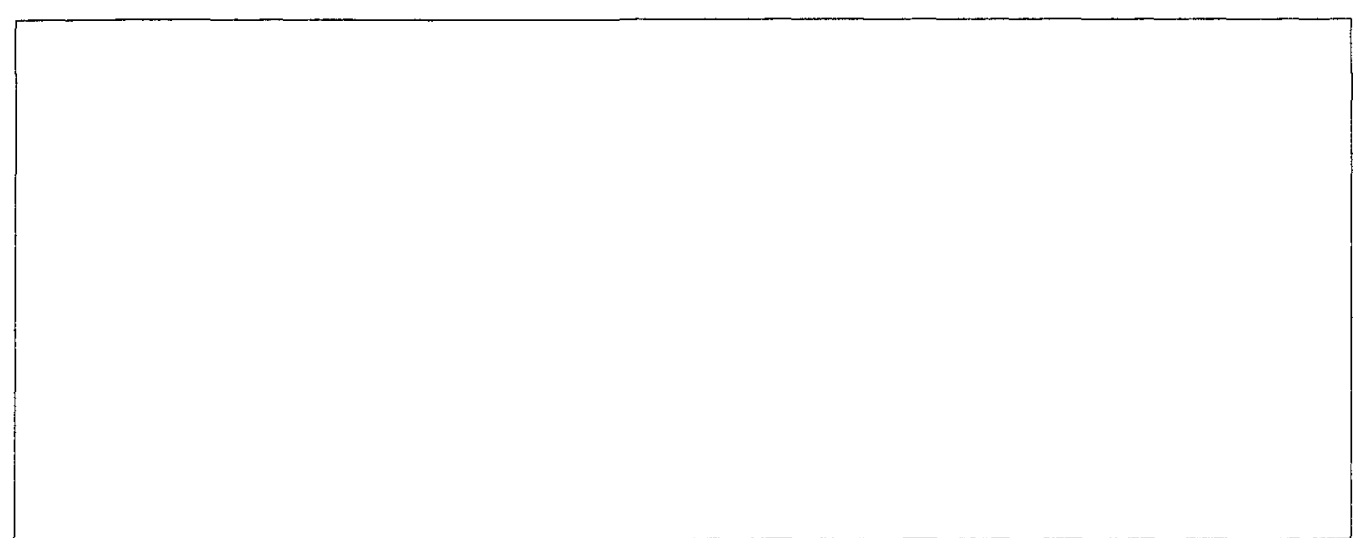

Figure A.4: The form for specifying the name and description when creating a new system. 
Fiease specify the XML file containing the bese configuration. Ensure the XML conforms to the Configuration DTD

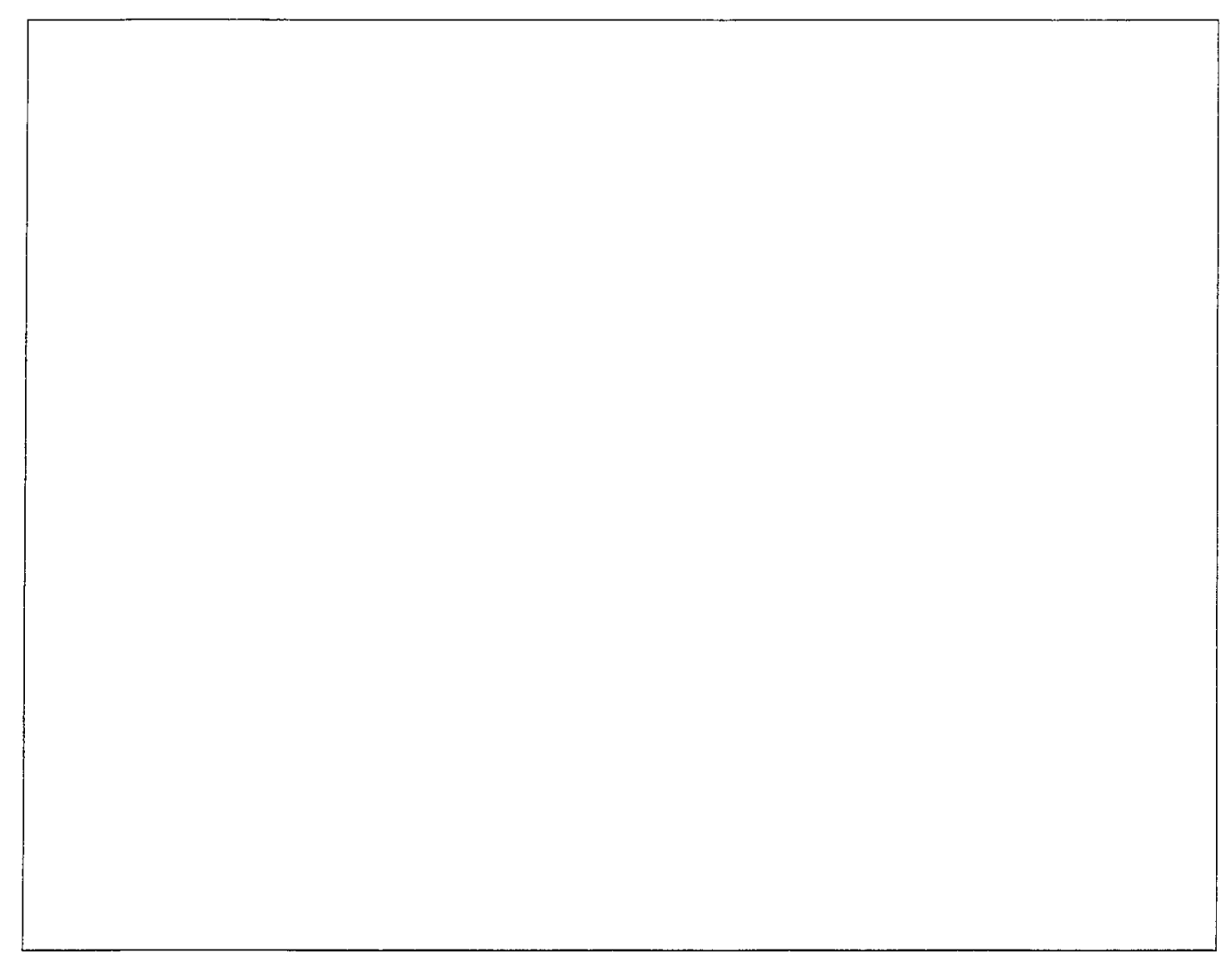

Figure A.5: The form for specifying the base configuration when creating a new system. 
APPENDIX A. DEPENDENCI AND CONSTRAINT MANAGEMENT TOOL243

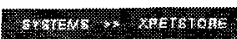

Retstore

DESCRIPTION

xPetstore appilication

COMFIGURATIONS

Listed below are all of the configurations that have been defined within this system To see a more detaled vew of a configuration, please clich on the appropriate link

xPetstore-Ease

Description: xFetstore-Ease description

xFetstare-Jboss

Description: *Petstore-Jboss description

xFetstore- JbossHSQL

Description: $x F$ etstore-Jboss $480 \mathrm{~L}$ description

xpetstore-onon

Description: $\times$ Petstore-Orion description

\&Fetstore-OrionPostgresal

Description: xFetstore-orionPostgresal description

Figure A.6: The client view of an existing system. 


\section{A.2.3 Configuration View}

Selecting a configuration from the specialization scheme presented in the system view will display the configuration view (Figure A.7). The configuration view lists all of the assemblies that are contained within a configuration.

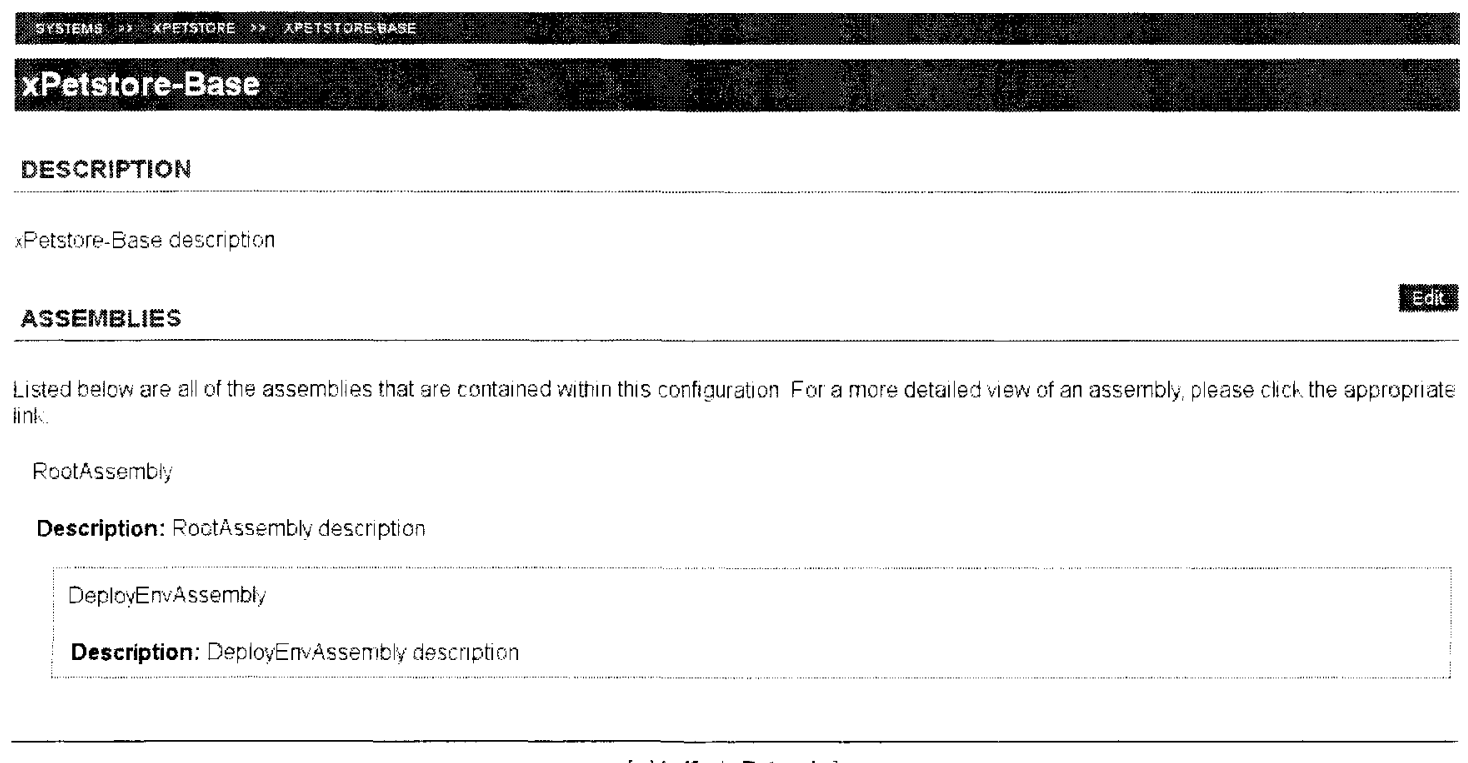

\section{[ Verify $\mid$ Extend ]}

Figure A.7: The client view of a configuration.

The configuration view uses different nesting levels to represent the configuration tree, and the hierarchical decomposition of the configuration. The root assembly begins the hierarchical decomposition, and it is located at the top, left-most position. Each nested assembly is the decompostion assembly of a composite component that is contained within the assembly at the next highest level in the tree. For example, the DeployEnvAssembly assembly is the decompostion assembly of a composite component contained within the RootAssembly assembly (Figure A.7).

It is possible to edit the description of a configuration by clicking the Edit button located to the right of the description text. 


\section{A.2.4 Specialize an Existing Configuration}

It is possible to create a specialization of a configuration by clicking on the Extend option located at the bottom of the configuration view. This will display the extend configuration page (Figure A.8 and Figure A.9).

To specialize a configuration, the XML representation of the specialization must be provided (Figure A.8). To assist in the process, the XML representation of the configuration being specialized is provided. This XML may be modified as necessary to create the specialization configuration.

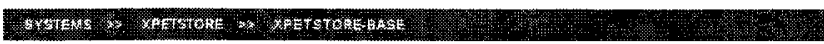

XPetstore-Base

EXTEND CONFIGURATION

Please enter the XML representation of the extended configuration in the text area below. Ensure that the XML conforms to the Configuration DTD

The XPA representation of the current configuration is given for your convenience. You may use this as a starting point Simply substitute the appropriate witual component with the desired concrete or composite component.

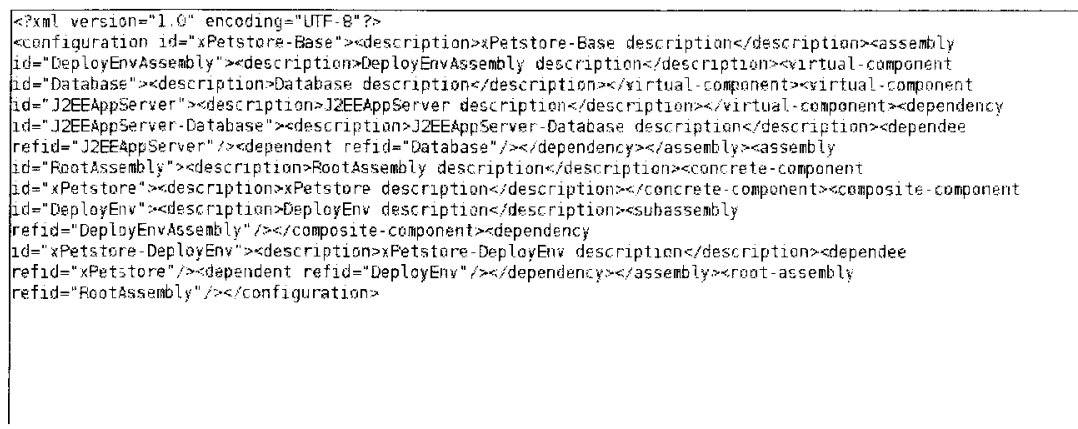

Figure A.8: The form for specifying the XML representation of a specialization.

It is necessary to provide a mapping that describes how the specialization was created (Figure A.9). The mapping must specify the virtual component that was replaced, and the concrete or composite component that has replaced the virtual component within the specialization configuration. All dependencies that had previously included the virtual component must also be mapped to the dependencies containing the newly inserted concrete or composite component. The mapping is specified using an XML document that must adhere to the mapping_1_O.dtd DTD. 


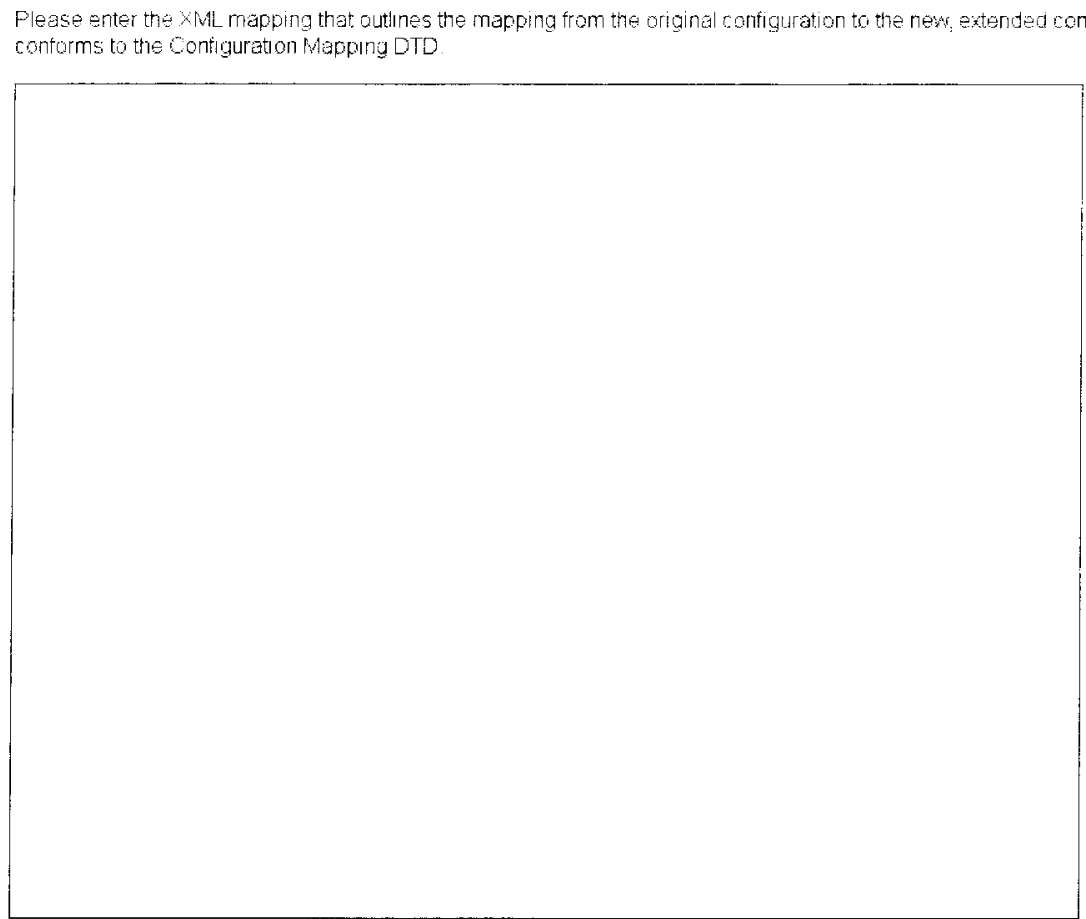

Figure A.9: The form for specifying the mapping from the configuration being specialized to the specialization.

\section{A.2.5 Assembly View}

Selecting an assembly from the list presented in the configuration view will display the assembly view (Figure A.10). The assembly view lists all of the components and dependencies contained within the assembly.

The name of each component contained in the assembly is given, along with the description of the component and the type of the component. Each composite component also contains a link to the sub-assembly containing the decomposition of the composite component (Figure A.10).

The name and description of each dependency contained within the assembly is also given in the assembly view.

It is possible to edit the description of the assembly by clicking the Edit button 


\section{DESCRIPTION}

Rootissembly description

COMPONENTS

Listed below are at of the components conteined within this assembly, In order to see the specifics of a component please click on the desired link

xPetstore

Description: «Petstore descriction

Type: Concrete

Deploy Env

Description: Deployeny description

Type: Composite

Sub-assembly: DeplovEnvAssembly

\section{DEPENDENCIES}

Listed below are all of the dependencies contained within this assembly in order to see the specifics of a dependency please click on the desired link xPetstore-DeployEn

Description: :Fetstore-Deplovenv descriotion

Figure A.10: The client view of an assembly. 
located to the right of the description text.

\section{A.2.6 Dependency View}

Selecting a dependency from the list presented in the assembly view will display the dependency view (Figure A.11). The dependency view displays the two components that comprise the dependency relationship, and it lists all of the constraints associated with the dependency.

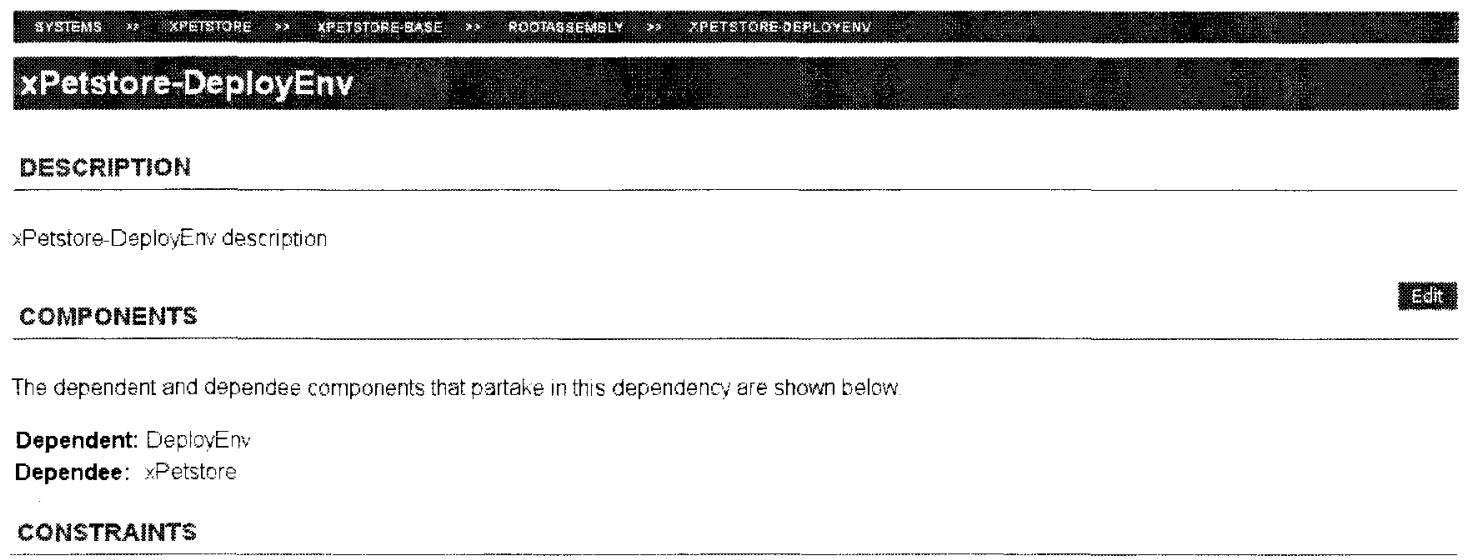

Listed below are all of the constrants associated with this dependency. For a more detailed vicw of each constrant please rick the app ropriate link.

- SampleConstraint

Figure A.11: The client view of a dependency.

It is possible to edit the description of the dependency by clicking the Edit button located to the right of the description text.

Constraints may be added to the dependency by clicking the Add button located to the right of the list of constraints that are currently associated with the dependency.

\section{A.2.7 Add New Constraint}

Adding a new constraint requires providing a name and description for the constraint (Figure A.12). The specified name must be unique within the configuration, and the 
description should provide a detailed explanation of the constraint being declared.
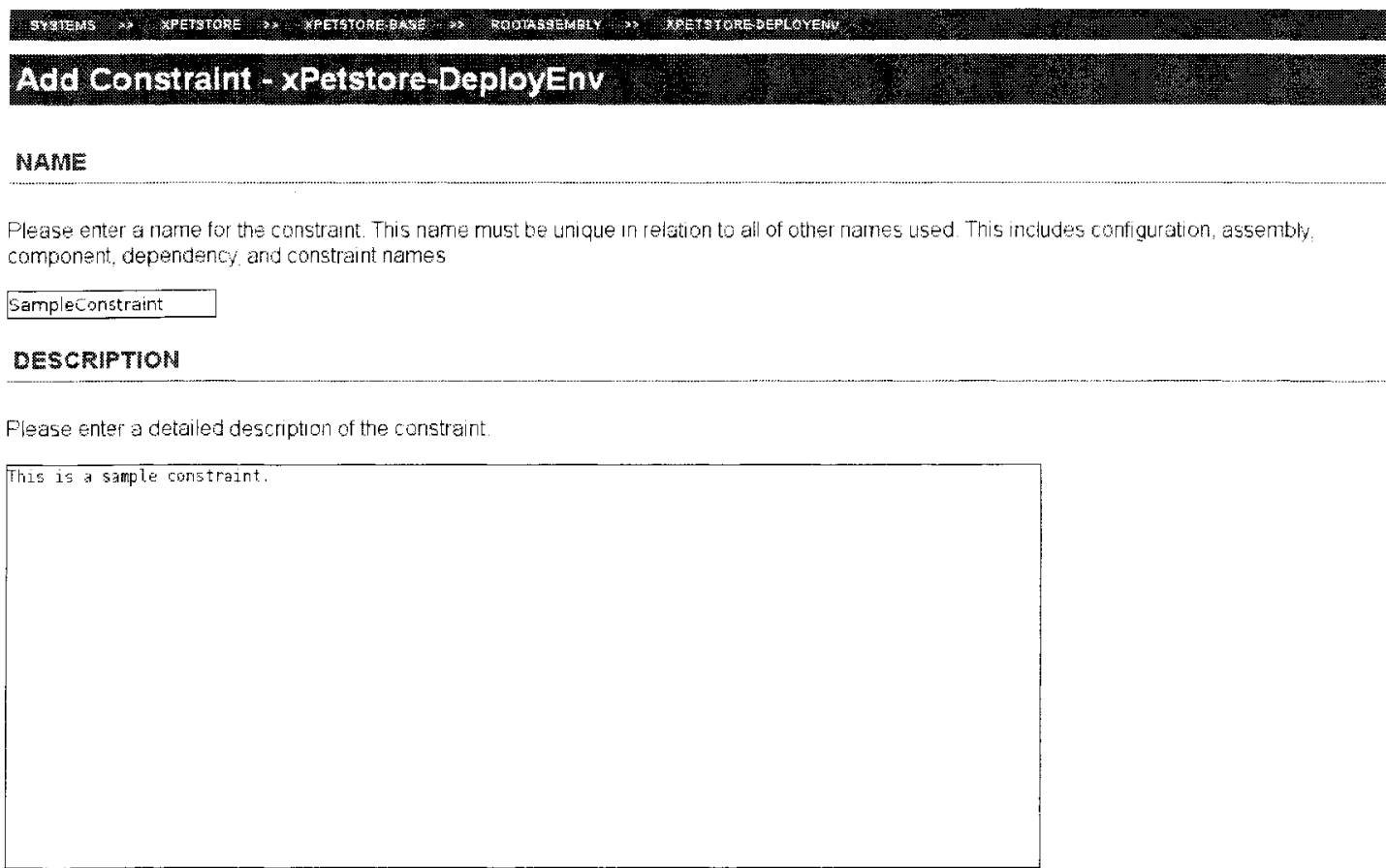

Figure A.12: The form for specifying the name and description of a new constraint.

If possible, a verification procedure should be defined (Figure A.13). The verification procedure should consist of a series of steps that describe how to determine whether or not the constraint has been properly satisfied.

\section{A.2.8 Constraint View}

Selecting a constraint from the list presented in the dependency view will display the constraint view (Figure A.14). The constraint view shows the textual description of the constraint and the verification procedure of the constraint, if it has been defined.

It is possible to edit the description of the constraint by clicking the Edit button located to the right of the description text.

It is possible to edit the verification procedure of the constraint by clicking the Edit button located to the right of the verification steps. 


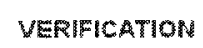

Please enter the verification procedure of the constraint, if one has been defined

Note: Enter each step of the procedure on a separate hne. and do not number the steps

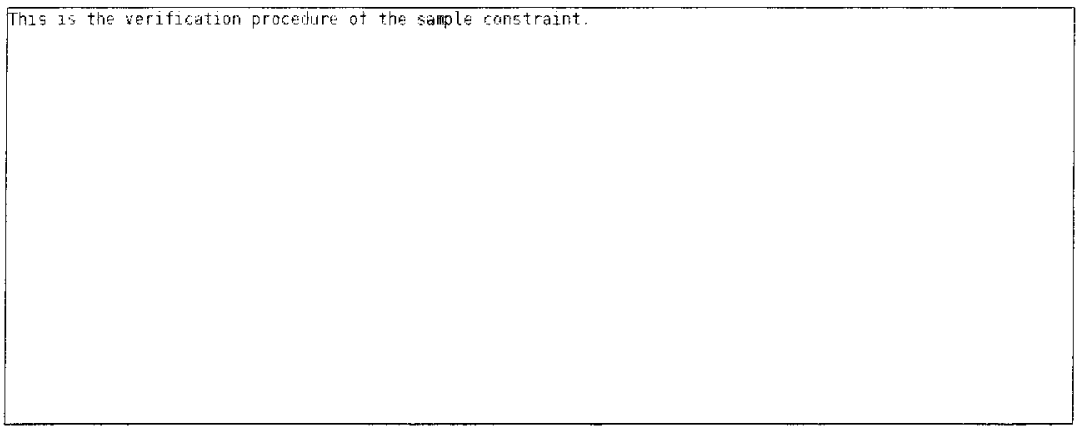

Figure A.13: The form for specifying the verification procedure of a new constraint.

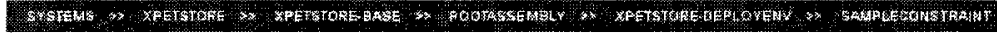

\section{Sampleconstraint}

\section{DESCRIPTION}

This is a sample constraint

VERPICATION

Eelow is the verification procedure that must be followed in order to deternme whether this constraint holds or not Flease follww these instructions carefully

1 This is the verfication procedure of the sample constrant.

Figure A.14: The client view of a constraint. 


\section{A.2.9 Committing Changes}

All changes that have been made to a configuration are maintained locally throughout the client session. In order for these changes to be stored permanently on the server. it is necessary to commit the changes. Changes may be committed by clicking the Commit option located at the bottom of the configuration view.

All pending changes for a configuration must be committed before a user can switch to another configuration. If the user decides not to commit the changes before switching between configurations, the changes will be lost.

As well, all pending changes for a configuration must be committed before a user can create a specialization of the configuration.

\section{A.3 Future Improvements}

This tool was developed as a proof of concept for providing tool support to the dependency and constraint management process. In order to advance the tool such that it may become integrated with the development and documentation environments of a software system there are several features and improvements that may be implemented:

- Develop a separate tool for graphically modeling and specializing configurations: In its current form, the tool requires that configurations be defined by manually creating the XML representation of the configuration. In order to facilitate this process, a separate tool for modeling the structure of the system could be implemented. The modeling tool would be based upon the formal specification defined in Chapter 4. As well, the modeling tool could be used to create spccializations of a configuration by generating the mapping XML file describing the changes made within the specialization.

- Generating a verification procedure report: The current implementation of the tool requires the user to manually view each constraint within a configuration in order to gather all of the verification procedure information. A verification 
procedure report could be generated for a configuration by combining all of the constraint information for a configuration into a single document.

- Link information between software products: Individual software products may be assembled into several larger software products. In order to prevent having to rediscover the dependencies and constraints contained within a software product each time that it is incorporated into a larger product, the tool should support linking between software products. Thus, when a software product is sclected to be used within an assembled product, the dependency and constraint information that has previously been defined for the software product may be linked to from the newly assembled product. This will allow for all dependency and constraint information pertaining to a single product to be maintained in a single location, and it may be referenced by all assembled products that include the product.

\section{A.4 configuration_1_0.dtd}

$<!--$

This is the DTD for configurations in the Dependency Modeling Tool.

$-->$

$<!--$

The configuration element is the root element.

$-->$

$<$ !ELEMENT configuration (description?, root-assembly, assembly+) > $<!--$

The description element allows for providing a description within the various elements.

Used in: configuration, assembly, concrete-component, virtual-component, composite-component, dependency, constraint

$->>$

$<$ !ELEMENT description (\#PCDATA) > 
$<!--$

The root-assembly is used for referencing the assembly within the configuration that is the root assembly.

Used in: configuration

$-->$

$<$ !ELEMENT root-assembly EMPTY $>$

$<!--$

The assembly element represents a single assembly within the

configuration. It contains all of the components and dependencies

included within the assembly.

Used in: configuration

$-->$

$<$ !ELEMENT assembly (description?, (concrete-component | composite-component

( virtual-component) + , dependency*) $>$

$<!--$

The concrete-component element represents a concrete component

within an assembly.

Used in: assembly

$->>$

$<$ !ELEMENT concrete-component (description?) >

$<!--$

The virtual-component element represents a virtual component within

an assembly.

Used in: assembly

$-\cdots$

$<$ !ELEMENT virtual-component (description?) $>$

$<!--$

The composite-component element represents a composite component within an assembly.

Used in: assembly 
$<$ !ELEMENT composite-component (description?, sub-assembly) $>$ $<!--$

The sub-assembly element is used to reference the assembly that is the expansion of a composite component.

Used in: composite-component

$-->$

$<$ !ELEMENT sub-assembly EMPTY $>$

$<!--$

The dependency element represents a dependency within an assembly.

Used in: assembly

$-->$

$<$ !ELEMENT dependency (description?, dependee, dependent, constraint + ) > $<!--$

The dependee element is used to reference the component that is the

'dependee' within a dependency. The dependee component must exist within

the same assembly as the dependency.

Used in: dependency

$->$

$<$ !ELEMENT dependee EMPTY $>$

$<!--$

The dependent element is used to reference the component that is the

'dependent' within a dependency. The dependent component must exist within

the same assembly as the dependency.

Used in: dependency

$->>$

$<$ !ELEMENT dependent EMPTY >

$<!--$

The constraint element represents a constraint that is associated with a

dependency.

Used in: dependency

$-->$ 
$<$ !ELEMENT constraint (description?, verification?) >

$<!--$

The verification element contains the verification procedure of a constraint.

Used in: constraint

$-->$

$<$ !ELEMENT verification (\#PCDATA) $>$

$<$ !ATTLIST configuration id ID \#IMPLIED $>$

$<$ !ATTLIST assembly id ID \#IMPLIED $>$

$<$ !ATTLIST concrete-component id ID \#IMPLIED $>$

$<$ !ATTLIST virtual-component id ID \#IMPLIED $>$

$<$ !ATTLIST composite-component id ID \#IMPLIED $>$

$<$ !ATTLIST dependency id ID \#IMPLIED $>$

$<$ !ATTLIST constraint id ID \#IMPLIED $>$

$<$ !ATTLIST root-assembly refid IDREF \#IMPLIED $>$

$<$ !ATTLIST sub-assembly refid IDREF \#IMPLIED $>$

$<$ !ATTLIST dependee refid IDREF \#IMPLIED $>$

$<!$ ATTLIST dependent refid IDREF \#IMPLIED $>$

\section{A.5 mapping_1_0.dtd}

$<!--$

This is the DTD for configuration mappings in the Dependency Modeling Tool.

It is used when extending a configuration to a more specialized configuration, and it describes the mapping from the original configuration to the extended configuration.

$->>$

$<!--$

The configuration-mapping element is the root element.

$-->$

$<$ !ELEMENT configuration-mapping (assembly-mapping + ) $>$ $<! \cdots$ 
The assembly-mapping clement describes the mapping from a single assembly in the original configuration to the appropriate assembly in the extended configuration.

Used in: configuration-mapping

$-->$

$<$ !ELEMENT assembly-mapping (componont-mapping*, dependency-mapping*) $>$ $<!--$

The component-mapping element describes the mapping from a single component in the original configuration to a component in the extended configuration. The component being mapped to must exist in the assembly that is mapped to by the assembly containing the original component.

Used in: assembly-mapping $->>$

$<$ !ELEMENT component-mapping EMPTY $>$ $<!--$

The dependency-mapping element describes the mapping from a single dependency in the original configuration to a dependency in the extended configuration. The dependency being mapped to must exist in the assembly that is mapped to by the assembly containing the original dependency. - - > $<$ !ELEMENT dependency-mapping EMPTY > $<$ !ATTLIST configuration-mapping name CDATA \#IMPLIED $>$ $<$ !ATTLIST configuration-mapping maps-to CDATA \#IMPLIED > $<$ !ATTLIST assembly-mapping name CDATA \#IMPLIED> $<$ !ATTLIST assembly-mapping maps-to CDATA \#IMPLIED > $<$ !ATTLIST component-mapping name CDATA \#IMPLIED> $<$ !ATTLIST component-mapping maps-to CDATA \#IMPLIED > $<$ !ATTLIST dependency-mapping name CDATA \#IMPLIED> $<$ !ATTLIST dependency-mapping maps-to CDATA \#IMPLIED> 


\section{Bibliography}

[1] O. Aoki. The Debian package management system. In Debian Reference, 2001.

[2] E. C. Bailey. Maximum RPM. Red Hat, Inc., 2000.

[3] Beagle. Available at: http://www.gnome.org/projects/beagle/.

[4] BeagleWiki. Available at: http://beaglewiki.org.

[5] Concurrent Versions System. Available at: http://www.gnu.org/software/ $\mathrm{cvs} /$.

[6] CORBA Components. Object Management Group, Inc., 2002.

[7] Deployment and Configuration of Component-based Distributed Applications Specification. Object Management Group, Inc., 2003.

[8] eCots - Software components open directory project. Available at: http://www . ecots.org.

[9] Hibernate. Available at: http://www.hibernate.org.

[10] hsqldb - 100\% Java database. Available at: http://hsqldb. sourceforge.net.

[11] IBM Software - DB2 product family. Available at: http://www-306.ibm.com/ software/data/db2/.

[12] IEEE standand for software configuration management plans, 1990.

[13] Java 2 Platform, Enterprise Edition. Available at: http://java.sun.com/j2ee. 
[14] Java 2 Platform. Standard Edition. Available at: http://java.sun.com/j2se.

[15] Java BluePrints > Enterprise BluePrints. Available at: http://java. sun.com/ blueprints/enterprise/index.html.

[16] JavaServer Pages Standard Tag Library. Available at: http://java. sun.com/ products/jsp/jstl.

[17] JavaServer Pages technology. Available at: http://java.sun.com/products/ $\mathrm{jsp} /$.

[18] JBoss.com. Available at: http://www.jboss.com.

[19] JDOM. Available at: http://www.jdom.org.

[20] A. Keller, U. Blumenthal, and G. Kar. Classification and computation of dependencies for distributed management. In Proceedings of Fifth IEEE Sypmosium on Computers and Communications, pages 78-83, Antibes-Juan les Pins, France, July 2000.

[21] MDA Guide Version 1.0.1. Object Management Group, Inc., 2003.

[22] Microsoft Corporation. Windows Installer Service Overview, 1999.

[23] Microsoft .NET. Available at: http://www.microsoft.com/net.

[24] J.-C. Mielnik, B. Lang, S. Lauriere, J.-G. Schlosser, and V. Bouthors. eCots Platform: An inter-industrial initiative for COTS-related information sharing. In Proceedings of Second International Conference on COTS-Based Software Systems, pages 157-167, Ottawa, Canada, February 2003.

[25] Mono. Available at: http://www.mono-project.com.

[26] MySQL Connector/J. Available at: http://www.mysql.com/products/ connector $/ \mathrm{j} /$.

[27] MySQL: The world's most popular open source database. Available at: http: //www. mysql. org. 
[28] Netscape.com. Available at: http://www.netscape.com.

[29] M. Northcott and M. Vigder. Managing dependencies between software products. In Proceedings of Fourth International Conference on COTS-Based Software Systems, pages 201-211, Bilbao, Spain, February 2005.

[30] Orion application server. Available at: http://www orionserver . com.

[31] PostgreSQL. Available at: http://www postgresql.org.

[32] PostgreSQL JDBC driver. Available at: http://jdbc.postgresql.org.

[33] Rational ClearCase. Available at: http://www-306.ibm.com/software/ awdtools/clearcase/.

[34] SQL server home. Available at: http://www.microsoft.com/sql/default. mspx.

[35] Subversion. Available at: http://subversion.tigris.org.

[36] M. Tepper. The rise of social software. netWorker, 7(3), September 2003.

[37] The Apache Struts web application framework. Available at: http://struts . apache.org.

[38] The Castor project. Available at: http://www. castor .org.

[39] The Jakarta site - the Jakarta project. Available at: http://jakarta.apache . org.

[40] W. Tresse. Open systems for collaboration. netWorker, 8(1), March 2004.

[41] Ubuntu. Available at: http://www.ubuntulinux.org/.

[42] A. van der Hoek and A. L. Wolf. Software release management for componentbased systems. In Software - Practice and Experience 93, January 2003.

[43] Velocity. Available at: http://jakarta.apache.org/velocity/index.html. 
[44] M. Vieira and D. J. Richardson. Analyzing dependencies in large componentbased systems. In Proceedings of the 17th IEEE International Conference on Automated Software Engineering, Edinburgh, UK, September 2002.

[45] M. Vieira and D. J. Richardson. The role of dependencies in component-based systems evolution. In Proceedings of IWPSE-02, 24th International Conference on Software Engineering, pages 62-68, Orlando, USA, May 2002.

[46] Visual SourceSafe. Available at: http://msdn.microsoft.com/vstudio/ previous/ssafe/.

[47] WikiWikiWeb front page. Available at: http://c2.com/cgi/wiki (Last accessed on April 18th, 2005).

[48] XDoclet: Attribute-Oriented Programming. Available at: http://xdoclet. sourceforge.net/xdoclet/index.html.

[49] xPetstore. Available at: http://xpetstore.sourceforge.net. 